\title{
Application of three-dimensional motion tracking of low-activity fiducial positron-emitting markers in radiation therapy and positron emission tomography
}

\author{
by \\ Marc Chamberland
}

A thesis submitted to the Faculty of Graduate and Postdoctoral Affairs in partial fulfilment of the requirements for the degree of

Doctor of Philosophy

in

Physics

Carleton University
Ottawa, Ontario

(ㅇ) 2015

Marc Chamberland 


\section{Abstract}

Patient body motion limits the delivery accuracy of radiotherapy and creates blurring artefacts on positron emission tomography (PET) images. Those adverse effects can be mitigated by tracking the patient body motion and using the information appropriately. A technique that can track the three-dimensional motion of low-activity positron-emitting fiducial markers was developed. The application of this tracking technique, called PeTrack, for respiratory-gated radiotherapy and for motion-compensated PET imaging was evaluated.

The feasibility of respiratory-gated radiotherapy using PeTrack was assessed. A respiratory gating interface was developed in LabVIEW to communicate with an Elekta Precise research linear accelerator and toggle the delivery of the beam. Radiochromic films were placed in a rod insert of an anthropomorphic dynamic thorax phantom to evaluate the dose distribution of the gated and non-gated delivery of a small square beam. A single low-activity source was used to track the motion of the phantom. Real patient breathing data were used as the basis of the motion of the phantom. Visual and quantitative assessments of the films confirmed that respiratory-gated radiotherapy using real-time tracking based on positron-emitting fiducial markers is achievable. The blurring of the dose distribution due to motion was greatly reduced on the gated deliveries compared to the non-gated cases.

A modified version of the tracking algorithm was developed to track fiducial markers when high physiological tracer activity from a patient undergoing PET imaging 
is present. Monte Carlo and phantom studies demonstrated that the tracking can achieve submillimetre accuracy and precision. The motion traces from the tracking were used to generated respiratory-gated cardiac PET images. The performance of PeTrack-based gating was compared to the Varian RPM system. PeTrack-based gating captured more motion of the patient and performed similarly to the RPM system. The feasibility of event-by-event motion correction of the raw PET data based on the tracking from PeTrack was also evaluated. Motion-corrected reconstructed images showed reduced motion blurring.

The potential for respiratory-gated radiotherapy and motion-compensated PET imaging using PeTrack was demonstrated. 
To Jezamine,

my love,

my light. 


\section{Acknowledgments}

To paraphrase the poet John Donne, no one is an island... and no one writes a thesis by themself.

First of all, I need to express my sincerest gratitude to my supervisor, Dr. Tong $\mathrm{Xu}$. He has been an incredible mentor and supervisor. I am eternally grateful for his unwavering support and guidance throughout my almost nine (!) years at his side. He is without a doubt the smartest person I have had the pleasure of knowing. His kindness, patience, positive attitude, and sense of humour have helped me get where I am today.

I am also grateful to all the people who discussed technical issues and offered their knowledge and their time with me: Dr. Charles Stearns from GE Healthcare, for his help with the D690 modelling; Dr. Kris Thielemans from UCL Hospital and Dr. Charalampos "Harry" Tsoumpas from the University of Leeds, for their guidance with image reconstruction tools; my colleague Chad Hunter, for his incredibly detailed knowledge of various aspects of PET imaging and helpful discussions; Dr. Joanna Cygler from The Ottawa Hospital Cancer Centre and Dr. Amanda Cherpak from the Nova Scotia Cancer Centre (formerly from The Ottawa Hospital Cancer Centre), for kindly providing real patient breathing data.

I am thankful for all my current and past colleagues in the Department of Physics. Thank you for making these years so memorable. In particular, my thanks to Ben 
Spencer for having shared my successes (and misfortunes!) in the PeTrack lab and to Simin Razavi for the friendship and support in the last years of my degree.

Thank you very much to Dr. Malcolm McEwen and Dr. Bryan Muir from the National Research Council of Canada for lending me their time and boundless expertise for my film measurements. My sincere thanks to Dr. Rob deKemp (and the Cardiac PET Centre staff) from the University of Ottawa Heart Institute for collaborating on the PET imaging part of my project and providing patient studies.

My deepest thanks to my friends and family who have always given me their love and support. Merci, Maman.

Finally, from the bottom of my heart, thank you to my best friend, my spouse, my love, Jezamine. Your love and belief in me carried me through those long and difficult years. But we finally did it. 


\section{Contributions}

This thesis presents the results of the research I have conducted throughout my $\mathrm{PhD}$ program at Carleton University. An abstract about Chapter 3 was submitted and accepted for an oral presentation to the World Congress on Medical Physics \& Biomedical Engineering that will be held in June 2015 in Toronto, Canada. I plan on submitting a manuscript based on this chapter to the American Institute of Physics journal Medical Physics. Parts of the results from Chapter 4 and Chapter 5 were presented at a poster session at the IEEE Nuclear Science Symposium \& Medical Imaging Conference in November 2014 in Seattle, USA. I plan on submitting a manuscript based on those results to the IEEE Transactions on Medical Imaging journal.

Sections 2.2.5 and 2.2.6 present work that I published as an article in Medical Physics. [1]. Part of that work was done during the course of my doctoral studies, but it was for the most part an extension of the research I conducted during my master's degree.

I wrote the EGSnrc user code to calculate dose and fluence, based on the EGSnrc example program tutor $7 p p$. Significant changes had to be made to the ausgab() function of tutor $r p p$ to allow scoring of dose and fluence to specific parts of the geometry. I ran all the simulations and analyzed all the data.

Dr. Tong Xu assisted me in the writing of the LabVIEW interface. Dr. Xu handled the components of the interface which establishes communication with the 
MPET units. I took care of the LabVIEW VIs which deliver and process the raw events from the detector system to the computer. I adapted the VIs from the open source program mesydaq. I designed and wrote all the LabVIEW VIs directly related to the gating of the Elekta linac. I also ported the PeTrack tracking code into a format compatible with LabVIEW. I added the normalized least mean squares adaptive filter to PeTrack to replace the previous prediction method (linear and circular interpolation). I designed the respiratory gating film measurement experiments at the National Research Council (NRC), with assistance and guidance from Dr. Xu. Dr. Malcolm McEwen and Dr. Bryan Muir from NRC helped integrate the PeTrack system to the Elekta linac. They operated the linac during the film measurements and scanned the radiochromic films. I conducted all the data analysis related to the measurements.

I studied the spatial distribution of coincidence events during PET imaging and determined the particular shapes of the distributions of the distance between events and marker. This was key for the tracking to succeed during PET imaging. I designed, ran, and analyzed all Monte Carlo simulations related to the development of the modified tracking algorithm. I also wrote the model of the GE Discovery 690 PET/CT scanner used in my simulations. Dr. Xu and I designed the phantom studies for motion tracking during PET imaging. Dr. Rob deKemp from the University of Ottawa Heart Institute (UOHI) helped set up the PET imaging phantom and operated the scanner during the studies. I analyzed all the data from the phantom studies. Dr. Rob deKemp and the PET imaging staff at the UOHI provided me with patient studies. I developed the respiratory gating method for PET imaging based on PeTrack. I also developed the event-by-event motion correction for PET imaging using PeTrack. When required, Dr. deKemp reconstructed the PET images from the GE Discovery 690 console. Dr. Ran Klein from The Ottawa Hospital produced the polar maps. I analyzed the resulting images and conducted all the data analysis. 


\section{Table of Contents}

Abstract $\quad$ ii

Acknowledgments $\quad$ v

Contributions vii

Table of Contents $\quad$ ix

List of Tables $\quad$ xiii

List of Figures $\quad$ xiv

List of Acronyms xxviii

List of Symbols $\quad$ xxxii

1 Introduction 1

1.1 Respiratory motion in radiation therapy . . . . . . . . . . . . . 1

1.2 Patient body motion in positron emission tomography imaging . . . . 2

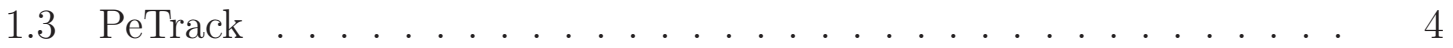

1.4 Thesis overview . . . . . . . . . . . . . . . . . . . . 4

2 Overview of PeTrack 5

2.1 Positron emission physics . . . . . . . . . . . . . . 5 
2.1.1 Positron emission . . . . . . . . . . . . . . . . 5

2.1.2 Positron annihilation . . . . . . . . . . . . 6

2.1.3 Scintillation detector . . . . . . . . . . . . . . . 7

2.1.4 Coincidence detection . . . . . . . . . . . . . . . . . . . . . . 7

2.1.5 Positron range and photon non-collinearity . . . . . . . . . 8

2.1.6 Time of flight . . . . . . . . . . . . . . 8

2.1.7 PET scanner . . . . . . . . . . . . . . . . . . . . . . . 10

2.1.8 Coincidence data storage . . . . . . . . . . . . . . . . . . . 11

2.2 PeTrack . . . . . . . . . . . . . . . . . . 15

2.2.1 Principle of operation . . . . . . . . . . . . . 15

2.2.2 Multimarker localization algorithm . . . . . . . . . . . . . 15

2.2.3 Initialization of the algorithm . . . . . . . . . . . . . 19

2.2.4 Fail-safe methods . . . . . . . . . . . . . . . . 20

2.2.5 Linear prediction . . . . . . . . . . . . . . . . . 21

2.2.6 Performance of the tracking algorithm . . . . . . . . . . . . 23

2.2.7 Fiducial markers . . . . . . . . . . . . . . . . 24

2.2.8 Prototype detector system . . . . . . . . . . . . . . . 24

2.2.9 Energy resolution . . . . . . . . . . . . . . . . . . 25

2.2.10 Crystal identification map . . . . . . . . . . . . . . . . . . 28

2.2.11 Sensitivity and accuracy of the prototype system . . . . . . 28

2.3 Conclusion . . . . . . . . . . . . . . . . . . . . . 31

3 Respiratory gating in radiation therapy using PeTrack 32

3.1 Introduction . . . . . . . . . . . . . . . . . 32

3.1.1 PeTrack for tumour tracking in radiotherapy . . . . . . . . . 36

3.2 Radiation environment of PeTrack in radiation therapy . . . . . . . . 37

3.2.1 EGSnrc simulation . . . . . . . . . . . . . . . 38 
3.2.2 Results........................ . . . 44

3.3 Experimental evaluation of respiratory gating with PeTrack . . . . . . 49

3.3.1 PeTrack linac gating software . . . . . . . . . . . . 52

3.3.2 Gating of the PeTrack detectors . . . . . . . . . . . . . . 54

3.3.3 Phantom motion, fiducial marker, and beam delivery . . . . . 57

3.3.4 Latency of the system . . . . . . . . . . . . . . 59

3.3.5 Results............................ 61

3.4 Discussion . . . . . . . . . . . . . . . . . . 70

3.4.1 EGSnrc simulation . . . . . . . . . . . . . . . 70

3.4.2 Respiratory gating ................ . . 70

3.5 Conclusion . . . . . . . . . . . . . . . . . . . 72

4 Motion tracking in PET imaging using PeTrack $\quad 74$

4.1 Introduction . . . . . . . . . . . . . . . . . . 74

4.1.1 PeTrack for motion tracking in PET imaging . . . . . . . . . . 75

4.2 The background problem . . . . . . . . . . . . . . 76

4.3 Modified initialization of the algorithm . . . . . . . . . . . 78

4.4 Background rejection . . . . . . . . . . . . . . 81

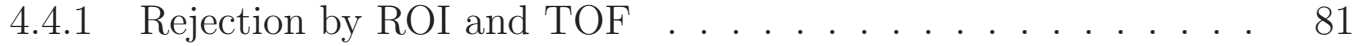

4.4.2 Determining the optimal ROI size . . . . . . . . . . . . 82

4.5 Flow of modified algorithm . . . . . . . . . . . . . . . 87

4.6 Evaluation using Monte Carlo simulations . . . . . . . . . . . . . . 89

4.6.1 Methods ..................... 89

4.6.2 Conversion from list mode to Cartesian coordinates . . . . . . 90

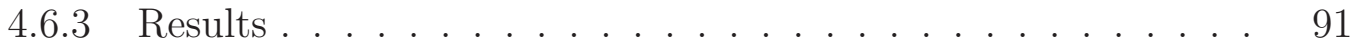

4.7 Experimental evaluation with a dynamic phantom . . . . . . . . . 96

4.7.1 Methods .......................... 96 
4.7.2 Results......................... . . . 98

4.8 Discussion . . . . . . . . . . . . . . . . . . . . . . . . . 104

4.9 Conclusion . . . . . . . . . . . . . . . . . 106

5 Motion compensation in PET imaging using PeTrack 107

5.1 Introduction . . . . . . . . . . . . . . . . . 107

5.2 PeTrack for motion compensation in PET imaging . . . . . . . . . . . 109

5.3 Human heart . . . . . . . . . . . . . . . . . . . . . . . . 110

5.4 Patient data . . . . . . . . . . . . . . . . . . . . 113

5.5 Respiratory gating using PeTrack . . . . . . . . . . . . . . . 114

5.5.1 Respiratory triggers from PeTrack . . . . . . . . . . . . . 114

5.5.2 Comparison of gating using RPM and PeTrack . . . . . . . . . 118

5.5.3 Results........................ 118

5.5 .4 Discussion . . . . . . . . . . . . . . . . . . . . 124

5.6 Motion correction using PeTrack . . . . . . . . . . . . . . . . . 126

5.6.1 Event-by-event motion correction . . . . . . . . . . . 126

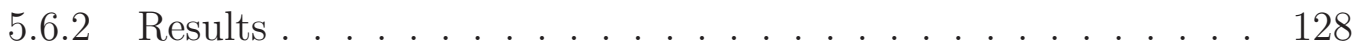

5.6 .3 Discussion . . . . . . . . . . . . . . . . 146

5.7 Conclusion . . . . . . . . . . . . . . . . . . . 150

6 Conclusions and future work $\quad 151$

6.1 Future work . . . . . . . . . . . . . . . 153

$\begin{array}{ll}\text { References } & 156\end{array}$ 


\section{List of Tables}

3.1 Parameters of each beam delivery. . . . . . . . . . . . . . . . 59

3.2 FWHM of profiles for each delivery. . . . . . . . . . . . . . 64

4.1 Geometry of the D690. The gap is the distance between two consecutive blocks or two consecutive crystals. . . . . . . . . . . . . . . . . 91

5.1 Statistics on the respiratory triggers from the Varian RPM system and from PeTrack (after user review). Note that user intervention for PeTrack added approximately 15 triggers to Anon4246 and 5 to Anon4886. Those additional triggers had a small effect on the overall statistics of the triggers. . . . . . . . . . . . . . . . . 120

5.2 FWHM of the fitted peaks of a line profile in the IS direction in the coronal plane for the three phantom scans. . . . . . . . . . . . . . 133 


\section{List of Figures}

2.1 Illustration of the different types of coincidences. a) True coincidence: two back-to-back photons created in the same annihilation event are detected in opposite detectors within the coincidence time window. b) Scattered coincidence: one or both of the photons are deflected due to scattering before being detected. c) Two photons from two different annihilation events are detected within the coincidence time window. The dashed lines in b) and c) represent the mispositioned LOR. . . .

2.2 Illustration of the errors (long dashed line) introduced by positron range (a) and photon non-collinearity (b) in the determination of the location of the positron emitting nucleus (dashed circle). . . . . . .

2.3 Illustration of principle of time of flight in coincidence detection. The distance $d$ is from the location of the annihilation event to the centre of the LOR. The path length difference between the two photons is $2 d$. If the difference in the time of detection of each photon is $\Delta t$, then the path length difference is given by $2 d=\Delta t \times c$ where $c$ is the speed of light in air. . . . . . . . . . . . . . . . . . 
2.4 The most common PET scanner configuration is the block ring configuration. Detector elements are arranged in arrays called blocks. (a) In the transaxial (or transverse) plane of the scanner, the blocks are distributed around the gantry in a regular polygon pattern. A detector element can be in coincidence with a fan of opposite detector elements. (b) In the axial plane of the scanner, detector elements can accept coincidences that are oblique (dashed line) as well as direct (solid line).

2.5 Geometric definition of the angular and radial bins in sinograms. Two coincidence photons detected by detector elements $\mathbf{D}_{1}$ and $\mathbf{D}_{2}$ are assigned a LOR defined by $r$ and $\theta \ldots \ldots \ldots \ldots$

2.6 Logical flow of the expectation-maximization algorithm. Modified from $[2] \ldots \ldots \ldots \ldots$

2.7 Prototype PeTrack system mounted on aluminum frame. . . . . . . 25

2.8 Energy spectrum of a single crystal element (size $3.9 \times 3.9 \times 20 \mathrm{~mm}^{3}$ ) exposed to radiation from a ${ }^{22} \mathrm{Na}$ source. The peak around channel 230 is the $511 \mathrm{keV}$ annihilation peak; the peak around channel 600 is the $1.274 \mathrm{MeV}$ photopeak of ${ }^{22} \mathrm{Na}$. This figure is reproduced from [3] with permission from the author. . . . . . . . . . . . 26

2.9 Energy spectrum of a detector module exposed to radiation from a ${ }^{22} \mathrm{Na}$ source. The peak near channel 375 is the $511 \mathrm{keV}$ annihilation

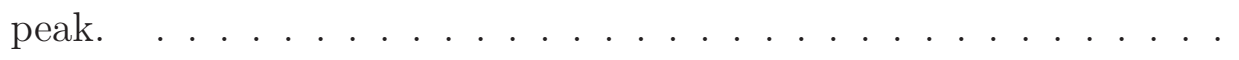


2.10 Sample 2D flood histogram from a prototype PeTrack detector block (see Section 2.2.8) exposed to radiation from a ${ }^{22} \mathrm{Na}$ source placed close to the surface of the detector. Each crystal element is clearly distinguishable with the exception of the four corner crystals, possibly due to lower sensitivity of the PMT in those regions. In addition, two crystals in the top row appear fused together. This may be due to a lack of reflective coating between the two crystals. Profiles of this figure along the horizontal (solid rectangle) and vertical (dashed rectangle) directions were measured. . . . . . . . . . . . . .

2.11 One dimensional profile of a row (a) and a column (b) of crystal elements, as measured on the 2D flood histogram (see Figure 2.10. All 12 crystals are clearly distinguishable along each direction, with a good peak-to-valley ratio. Non-uniform gain of the PMT causes the crystals in the centre to have a lower intensity than crystals closer to the edges. 30

3.1 PeTrack detector modules (A1, A2, B1, and B2) mounted on a linac gantry. Reproduced with permission from AAPM. . . . . . . . . 37

3.2 Design of the detector blocks. Figures are not to scale. . . . . . . . . 39

3.3 Design of the modules and final geometry placement. Figures are not to scale. . . . . . . . . . . . . . . . . .

3.4 Average dose to the upper modules relative to the lower modules (top left) and average dose to the lower modules relative to the maximum dose along the central axis of the phantom (bottom left) for various nominal beam energies and field size $10 \times 10 \mathrm{~cm}^{2}$. The same quantities for nominal beam energy $10 \mathrm{MV}$ and various field sizes are shown on the right. . . . . . . . . . . . . . . . 
3.5 Fluence at the centre of the aluminum window of the upper modules (a) and lower modules (b) for energies up to $1 \mathrm{MeV}$, in $2 \mathrm{keV}$ wide bins. The fluence was normalized to the total fluence of the source. In EGS++, the fluence of a parallel beam source is defined as the total number of simulated histories divided by the area of the shape of the beam. The peaks at $77 \mathrm{keV}$ and $87 \mathrm{keV}$ correspond to the characteristic $\mathrm{x}$ rays of bismuth. The peak at $511 \mathrm{keV}$ is an annihilation peak. For the sake of visual clarity, five data points are skipped between each point on the graph. . . . . . . . . . . . . . . .

3.6 Fluence at the centre of the aluminum window of the upper modules (a) and lower modules (b) for energies between 1 and $10 \mathrm{MeV}$, in $250 \mathrm{keV}$ bins. The fluence was normalized to the fluence of the source. In EGS++, the fluence of a parallel beam source is defined as the total number of simulated histories divided by the area of the shape of the

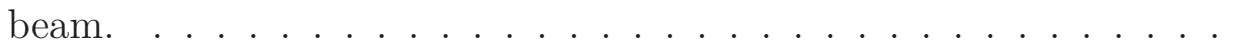

3.7 The prototype PeTrack detectors mounted on an aluminum frame under the head of a linac. An anthropomorphic dynamic thorax phantom sits on the treatment table. . . . . . . . . . . . . . 50

3.8 Overview of the experimental setup. The two MPET-8 modules acquire coincident events from the two pairs of detector modules. The MCPD-2 collects the events and sends them to a PC. Each detector module is equipped with a high voltage gating device which turns the PMT HV off during every linac x-ray pulse based on a pulsing signal from the linac, regulated by a pulse generator. An electrometer reads out the signal from the ion chamber. The reading from the electrometer is sent out to a $\mathrm{PC}$, through a digital-to-analog converter. . . . . . . . 
3.9 The GUI of the tracking component allows users to control various parameters such as the sampling frequency and the prediction order. .

3.10 The GUI of the gating component allows users to set the size of the gating window and monitor the status of the beam delivery. . . . .

3.11 PMT output due to scattered $\mathrm{x}$ rays from a $6 \mathrm{MV}$ photon beam incident on a $30 \times 30 \times 22 \mathrm{~cm}^{3}$ water phantom for various field sizes. As the field size increases, more scattered $\mathrm{x}$ rays reach the scintillator crystal coupled to the PMT. The signal from the PMT should increase with the field size. However, the figure shows that the PMT output varies very little with the field size. This indicates that the PMT is saturated by the scintillation light from the scattered radiation. This figure was reproduced from $[3]$ with permission from the author. . . . . . .

3.12 Sample oscilloscope traces illustrating the gating of the PeTrack detectors during linac operation. Linac pulse indicator signal: this pulse leads every $\mathrm{x}$ ray pulse; it is generated by the PFN and starts before actual $x$ rays are produced. PMT gating pulse: the linac pulse indicator signal triggers a signal generator to produce a square PMT gating pulse. PMT voltage: the PLL synchronizes with the linac pulse indicator signal and generates a high voltage gating window $90 \mu$ s wide, roughly centred on the PMT gating pulse. . . . . . . . . . . . .

3.13 Radiochromic films were placed within the rod and marked for registration. A small fiducial marker was placed at the superior end of the rod, in a source holder. . . . . . . . . . . . . . . . 
3.14 Position of the marker in the IS direction (top) and status of the beam (hold or on; bottom) throughout the delivery with sinusoidal translation of the rod. The dashed area represents the gating window within which the beam is on. Note that approximately 6 minutes into the delivery of the beam, the beam inadvertently turned off due to an operator mistake. The beam was promptly turned back on. . . . . . .

3.15 Position of the marker in the IS direction and status of the beam (hold or on) throughout the deliveries with real patient breathing motion. The dashed area represents the gating window within which the beam is on. Note that at various moments (for example, at around 7 minutes on subfigure a)), the marker moves out of the gating window soon after first entering it, then subsequently re-enters the gating window. This causes the beam to turn on, off, and on again in quick succession, in accordance with the design of the gating system. . . . . . . . . .

3.16 Predicted and current position of the marker at this time (top) plotted with the current in the ion chamber (bottom). The dashed area represents the gating window. Note that the predicted location of the marker has been shifted by one localization interval. In other words, at any given time $t_{n}$, the current curve gives the current location of the marker whereas the predicted curve gives the predicted location of the marker at time $t_{n+1}$, based on the current location and 29 previous locations of the marker before $t_{n} \ldots \ldots \ldots$

3.17 Radiochromic films after exposure to $500 \mathrm{MU}$ of a $3 \times 3 \mathrm{~cm}^{2}$ photon beam at nominal energy $6 \mathrm{MV}$. The phantom rod was translated sinusoidally in the IS direction. Line profiles in the IS direction (indicated by the arrows) were measured and plotted in Figure 3.19. . . . . . . 
3.18 Radiochromic films after exposure to $500 \mathrm{MU}$ of a $3 \times 3 \mathrm{~cm}^{2}$ photon beam at nominal energies 6 and $10 \mathrm{MV}$. The translation of the phantom rod in the IS direction was based on real patient breathing data. Line profiles in the IS direction (indicated by the arrows) were measured and plotted in Figure 3.20. . . . . . . . . . . . . . . . 67

3.19 Line profiles across the dose distribution in the IS direction for films exposed under sinusoidal motion. The values are normalized to the maximum of the reference profile, after subtracting their individual background. . . . . . . . . . . . . . . . .

3.20 Line profiles across the dose distribution in the IS direction for films exposed under real patient breathing motion. The values are normalized to the maximum of the reference profile, after subtracting their individual background . . . . . . . . . . . . . . .

4.1 Top: Number of events detected in $0.5 \mathrm{~s}$ bins during a dynamic cardiac PET scan. The patient was injected with $840 \mathrm{MBq}$ of ${ }^{82} \mathrm{Rb}$. Bottom: Proportion of the total detected events (in $0.5 \mathrm{~s}$ bins, since PeTrack will track the marker every $0.5 \mathrm{~s}$ ) that came from a single $2.5 \mu \mathrm{Ci}$ marker placed on the patient's abdomen. . . . . . . . . . . . . . . . 77

4.2 A CT-visible PeTrack fiducial marker is placed on a patient's abdomen. Its location on the attenuation correction CT scan can be used to initialize the tracking algorithm. . . . . . . . . . . . .

4.3 A PeTrack fiducial marker is visible on a transverse slice of a dynamic PET series. This image is the first frame in a series of 32 15-s frames. The activity of the marker is approximately $92.5 \mathrm{kBq} . \quad$. . . . . .

4.4 The distribution (0.05 $\mathrm{mm}$ bin width) of the perpendicular distance between a marker and the LORs from the background (90 $\mu \mathrm{Ci}$ activity). 
4.5 Distribution of the perpendicular distance $(0.05 \mathrm{~mm}$ bin width) between a marker and all LORs. Events under the peak are expected to come from the marker. Events in the tail are a combination of events from the marker ( $40 \mu \mathrm{Ci}$ activity) and from the background (90 $\mu \mathrm{Ci}$ activity). . . . . . . . . . . . . . . .

4.6 Illustration of the method to determine the size of the ROI for rejecting background events. The solid (black) histogram shows the distance between the estimated location of the marker and all coincidence events. A linear fit (solid blue line) is performed on the tail of this distribution and is extrapolated to zero distance (dashed blue line). The values of the fit at the centre of each bin are subtracted from the content of each bin. The dashed (red) histogram shows the value of each bin after subtraction of the background fit. The size of the ROI is set to the centre of the first bin beyond the peak with a value $\leq 0$; the arrow points to this bin on the figure. Note that this histogram is generated for every run of localization. . . . . . . . . . . . . .

4.7 Illustration of the background rejection technique. For simplicity, only two opposite rows of PET detectors are shown. a) ROI rejection: any event that is more than $30 \mathrm{~mm}$ away from the initial estimate of the marker's location is rejected. The ROI is represented by the dotted circle. b) TOF rejection: events whose estimated annihilation location is more than $80 \mathrm{~mm}$ from the marker are also rejected. c) Fitting for the optimal ROI size: the size of the ROI is adjusted by the fitting method. d) The ROI and TOF rejection methods are applied once

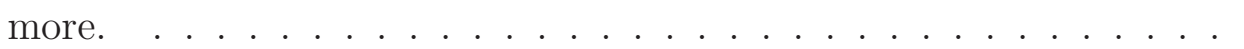


4.8 Fraction of the events used by the algorithm that originated from the marker. Initially (black squares), the vast majority of events are from the patient background activity. The fraction is increased by a factor 100 after rejecting events based on a $30-\mathrm{mm}$ radius $\mathrm{ROI}$ and TOF data (red circles). Near the peak of the TAC, another increase by a factor 25 is achieved by optimizing the size of the ROI through linear fitting. Note: every sixth data point is shown for visual clarity purposes. .

4.9 Location of the simulated marker (data points) along the three anatomical directions, as calculated by the PeTrack algorithm. The real location of the marker is shown by the solid red line and is defined by

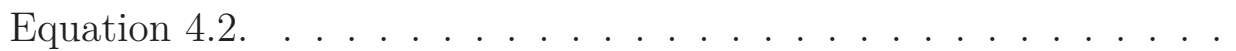

4.10 Distribution of the 3D error. The 3D error is the distance between the location of the marker $\mathbf{r}(t)$ and the location calculated by PeTrack at time $t$. The average of the distribution is $(0.8 \pm 0.3) \mathrm{mm}( \pm 1 \mathrm{SD})$. .

4.11 Anthropomorphic torso phantom used in the study. Three external fiducial markers are held onto the surface of the phantom with modelling clay. The main cavity of the phantom was filled with water and the cardiac insert was filled with a ${ }^{82} \mathrm{Rb}$ solution. The phantom was placed on a motor-driven platform. The block with reflective markers used by the Varian RPM system was attached to the rod. . . . . .

4.12 Motion traces of a sample marker for the static (\#1), slow (\#2), and fast and shifting (\#3) scans. . . . . . . . . . . . . .

4.13 Distribution of the 3D distance between the calculated location of the marker and its average location throughout the scan. The mean of the distribution is $(0.26 \pm 0.14) \mathrm{mm}$. The precision of the tracking can be taken as the standard deviation of this distribution, i.e. $0.14 \mathrm{~mm}$. . . 101 
4.14 Distribution of the relative accuracy among all three scans. The relative accuracy is defined as the difference between the real distance between a pair of markers and the distance as calculated from the location of the markers determined by the algorithm. According to this definition, a negative relative accuracy indicates that the real distance between the markers is smaller than distance calculated from the location of the markers provided by PeTrack. The mean of the distribution

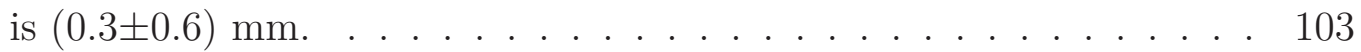

5.1 Illustration of the principle of phase gating. Respiratory triggers (arrows) correspond to the time of end inhale, in this example. The breathing cycle is divided into eight equal size temporal gates between two consecutive triggers. Events are retrospectively binned into those eight gates. . . . . . . . . . . . . . . . . . . . . . . . . 108

5.2 The three standard cardiac imaging planes of the heart: A) the horizontal long axis (HLA); B) the vertical long axis (VLA); and C) the short axis (SA). This figure is reproduced from Choi et al [4] with permission under a Creative Commons Attribution-NonCommercial 3.0 Unported License (CC BY-NC 3.0; http://creativecommons.org/licenses/bync/3.0/); copyright (C) 2006 The Korean Society of Radiology. . . . . . 111 
5.3 Anatomy of the human heart. Major coronary arteries are labeled in red; other landmarks are in blue or black. This figure is reproduced with permission under a Creative Commons Attribution-Share Alike 3.0 Unported License (CC BY-SA 3.0; http://creativecommons.org/licenses/by-sa/3.0/). Original figure Coronary.pdf by Patrick J. Lynch, medical illustrator; derivative work by Fred the Oyster; adaptation and further labeling by Mikael Häggström. Additional labels in black were added to this version by Marc Chamberland. .................... 112

5.4 Diagram showing the coronary arteries, landmarks, and anatomical directions corresponding to the areas of a polar map. LAD: left anterior descending artery; LCX: left circumflex artery; RCA: right coronary

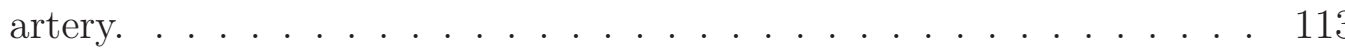

5.5 Motion trace of the marker in each direction for each study. Anon4246 was administered $\mathrm{CO}_{2}$ three minutes before tracer injection, until three minutes post injection. Anon4886 received no $\mathrm{CO}_{2}$. . . . . . . .

5.6 Motion trace of the marker in each direction for each study. Anon4246 was administered $\mathrm{CO}_{2}$ three minutes before tracer injection, until three minutes post injection. This explains the change in breathing amplitude at the 3 -minute mark. Anon4886 received no $\mathrm{CO}_{2} \ldots \ldots$. . . . . . 117

5.7 Histograms of the distribution of the breathing interval for both gating systems. . . . . . . . . . . . . . . . . . . . . . . . 119

5.8 Comparison of the PeTrack respiratory triggers (identified as the peaks of the motion trace; short red vertical dashes) and the RPM triggers (blue vertical dashed lines) for Anon4246. . . . . . . . . . . . . . . . 121 
5.9 Comparison of the PeTrack respiratory triggers (identified as the peaks of the motion trace; red vertical dashes) and the RPM triggers (blue vertical solid lines) for Anon4886. . . . . . . . . . . . . . .

5.10 Comparison of the polar maps of the average perfusion of the LV for the respiratory gated series of Anon4246 and Anon4886. . . . . . . . 123

5.11 Illustration of the motion correction of events. The motion correction vector at time $t, \boldsymbol{\Delta}_{M C}(t)$, is defined as the difference between the reference marker position and the current marker position. The coincidence event $\mathbf{L}$ gets transformed to $\mathbf{L}^{M C}$. . . . . . . . . . . . 127

5.12 Sample coronal slice of the non-dynamic series for each phantom scan. Scan \#1 is the reference static scan. . . . . . . . . . . . .

5.13 Comparison of non-motion-corrected and motion-corrected sample slices along the short axis (SA) and horizontal long axis (HLA) of the heart for the static reference scan (scan \#1). Note the air bubble near the apex of the heart, on the HLA slice. . . . . . . . . . . . . 129

5.14 Comparison of non-motion-corrected and motion-corrected sample slices along the short axis (SA) and horizontal long axis (HLA) of the heart for scans \#2 and \#3. Note the air bubble near the apex of the heart, on the HLA slice. . . . . . . . . . . . . . . . . . 130

5.15 Measured line profiles in the coronal plane along the IS direction. Each peak was fitted with a Gaussian model. Scan \#1 is the reference static scan. . . . . . . . . . . . . . . . . . . . . . . 132

5.16 Non-MC and MC traces of the marker for phantom scan \#2. Note the unchanged LR and AP traces after motion correction and the discontinuities in the IS direction; those will be explained in Section 5.6.3. 
5.17 Non-MC and MC traces of the marker for phantom scan \#3. The shift between the LR traces and the AP traces before and after motion correction is caused by choosing the reference position of the marker on a CT image. The position of the marker on the CT image was slightly offset from the position of the marker on the PET image. . . . . . . . 136

5.18 Comparison of the polar maps of the average perfusion of the LV for phantom scans \#2 and \#3. Scan \#3 also includes a polar map of the detected wall motion by an automatic segmentation algorithm. Note that the non-uniformity of the perfusion near the apex of the heart is most likely due to an air bubble in the heart insert (compare to Figure 5.14). . . . . . . . . . . . . . . . . . . . 137

5.19 Non-MC and MC traces of the marker for Anon4886. . . . . . . . . . 139

5.20 Line profile across the marker and along the LR direction in the transverse plane of Anon4886. The AP direction (vertical line) is not used.

5.21 Measured line profile across the marker and along the LR direction in the transverse plane of Anon4886. A Gaussian model was fitted to the profile.

5.22 Non-MC and MC traces of the marker for Anon3512. . . . . . . . . 143

5.23 Sample transverse slice of the non-motion-corrected dynamic series for Anon3512. The series increases in time by $15 \mathrm{~s}$ in each frame (left to right, top down). A reference line (dashed) was drawn through the location of the fiducial marker during the first frame. . . . . . . . . . 144

5.24 Sample transverse slice of the motion-corrected dynamic series for Anon3512. The series increases in time by $15 \mathrm{~s}$ in each frame (left to right, top down). A reference line (dashed) was drawn through the location of the fiducial marker during the first frame. . . . . . . . 
5.25 The motion correction is limited to correcting to the nearest crystal because the GE list mode format stores coincidence events as pairs of crystal indices. a) Events in the list mode are associated to the nearest crystal. An event has to move by half the crystal pitch before moving to the adjacent crystal pair. b) Close up of Figure 5.16. The discreteness of the motion correction causes discontinuities in the motion-corrected trace of the marker. . . . . . . . . . . . . . . . . . . . . 148

5.26 In the transaxial plane of the scanner, the distance between adjacent parallel coincidence events decreases away from the central axis. . . . 149 


\section{List of Acronyms}

\begin{tabular}{ll}
\hline Acronyms & Definition \\
\hline \hline 1D & one dimensional \\
2D & two dimensional \\
3D & three dimensional \\
AAPM & American Association of $\mathrm{Physicists} \mathrm{in}$ Medicine \\
ADC & analog-to-digital converter \\
AP & anterior posterior \\
BGO & bismuth germanate $\left(\mathrm{Bi}_{4} \mathrm{Ge}_{3} \mathrm{O}_{12}\right)$ \\
CAD & coronary artery disease \\
COIL & continuous slowing down approximation \\
&
\end{tabular}




$\begin{array}{ll}\text { D690 } & \text { GE Discovery } 690 \text { PET/CT scanner } \\ \text { EBE } & \text { event-by-event } \\ \text { EGRT } & \text { emission guided radiotherapy } \\ \text { EM } & \text { expectation-maximization } \\ \text { 18FDG } & { }^{18} \text { F-fluorodeoxyglucose } \\ \text { FOV } & \text { field of view } \\ \text { FPGA } & \text { field-programmable gate array } \\ \text { FWHM } & \text { full-width at half-maximum } \\ \text { GATE } & \text { GEANT4 Application for Tomographic Emission } \\ \text { GSO } & \text { gadolinium oxyorthosilicate } \\ \text { GUI } & \text { graphical user interface } \\ \text { LCX } & \text { light emitting diode } \\ \text { HE } & \text { internal treatment volume } \\ \text { ICRU } & \text { international Commission on Radiation Units and Measurements } \\ \text { IS } & \text { inferior superior } \\ & \end{array}$

xxix 


\begin{tabular}{|c|c|}
\hline linac & linear accelerator \\
\hline LOR & line of response \\
\hline LR & left right \\
\hline LV & left ventricle \\
\hline LYSO & lutetium yttrium orthosilicate \\
\hline MAF & multiple acquisition frame \\
\hline $\mathrm{MC}$ & motion corrected \\
\hline MLC & multileaf collimator \\
\hline MPI & myocardial perfusion imaging \\
\hline MRI & magnetic resonance imaging \\
\hline MU & monitor unit \\
\hline MV & mega voltage \\
\hline nLMS & normalized least mean squares \\
\hline NRC & National Research Council of Canada \\
\hline $\mathrm{PDF}$ & probability density function \\
\hline PET & positron emission tomography \\
\hline PeTrack & Positron Emission Tracking \\
\hline PFN & pulse forming network \\
\hline PLL & phase-lock loop \\
\hline
\end{tabular}




\begin{tabular}{|c|c|}
\hline PMT & photomultiplier tube \\
\hline $\mathrm{PRF}$ & pulse repetition frequency \\
\hline PTFE & polytetrafluoroethylene \\
\hline $\mathrm{RCA}$ & right coronary artery \\
\hline RMS & root mean square \\
\hline RMSE & root mean square error \\
\hline (Varian) RPM & (Varian) Real-time Position Management ${ }^{\mathrm{TM}}$ system \\
\hline ROI & region of interest \\
\hline $\mathrm{RR}$ & Russian roulette \\
\hline SSD & source-surface distance \\
\hline SNR & signal-to-noise ratio \\
\hline $\mathrm{TAC}$ & time activity curve \\
\hline TCP & tumour control probability \\
\hline $\mathrm{TOF}$ & time of flight \\
\hline TOR & tube of response \\
\hline UOHI & University of Ottawa Heart Institute \\
\hline VOI & volume of interest \\
\hline VRT & variance reduction technique \\
\hline
\end{tabular}




\section{List of Symbols}

\begin{tabular}{|c|c|}
\hline Symbols & Definition \\
\hline $\boldsymbol{\alpha}(n)$ & vector of linear prediction coefficients \\
\hline$\alpha_{k}$ & relative activity of marker $k$ \\
\hline$A$ & amplitude of motion or mass number \\
\hline$\beta^{+}, e^{+}$ & positron \\
\hline$c$ & speed of light in vacuum \\
\hline$d$ & $\begin{array}{l}\text { distance from the annihilation location to the centre of a } \\
\text { LOR in TOF PET }\end{array}$ \\
\hline$d \bar{\epsilon}$ & average energy imparted to element of mass $d m$ \\
\hline$d m$ & element of mass \\
\hline$\vec{d}\left(\mathbf{L}_{n}, \vec{m}_{k}\right)$ & $\begin{array}{l}\text { minimum distance vector from coincidence line } \mathbf{L}_{n} \text { to } \\
\text { marker location } m_{k}\end{array}$ \\
\hline$D$ & absorbed dose \\
\hline$D_{1}, D_{2}$ & transaxial detector element indices \\
\hline${ }_{Z}^{A} D_{N}$ & daughter element \\
\hline
\end{tabular}




\begin{tabular}{|c|c|}
\hline$\Delta_{M C}(t)$ & motion correction vector at time $t$ \\
\hline$e^{-}$ & electron \\
\hline$e(n)$ & prediction error in the nLMS adaptive filter algorithm \\
\hline$\epsilon$ & efficiency of a Monte Carlo simulation \\
\hline$\epsilon_{t o t}$ & total energy imparted in a volume \\
\hline$G\left(\left|\vec{d}\left(\mathbf{L}_{n}, \vec{m}_{k}\right)\right|, \sigma_{k}\right)$ & $\begin{array}{l}\text { probability density function of the Gaussian distribution } \\
\text { of the minimum distance between a marker and its coinci- } \\
\text { dence lines }\end{array}$ \\
\hline$\gamma$ & photon (gamma ray) \\
\hline$\Gamma$ & $\begin{array}{l}\text { a small positive constant in the nLMS adaptive filter algo- } \\
\text { rithm }\end{array}$ \\
\hline$i$ & iteration index \\
\hline$k$ & marker index \\
\hline$K$ & total number of markers \\
\hline$l$ & track length of particles depositing energy in a volume \\
\hline $\mathbf{L}$ & coincidence line \\
\hline $\mathbf{L}^{M C}$ & motion corrected coincidence line \\
\hline$m_{e}$ & electron mass \\
\hline$m_{k}$ & location of marker $k$ \\
\hline M & total mass \\
\hline
\end{tabular}




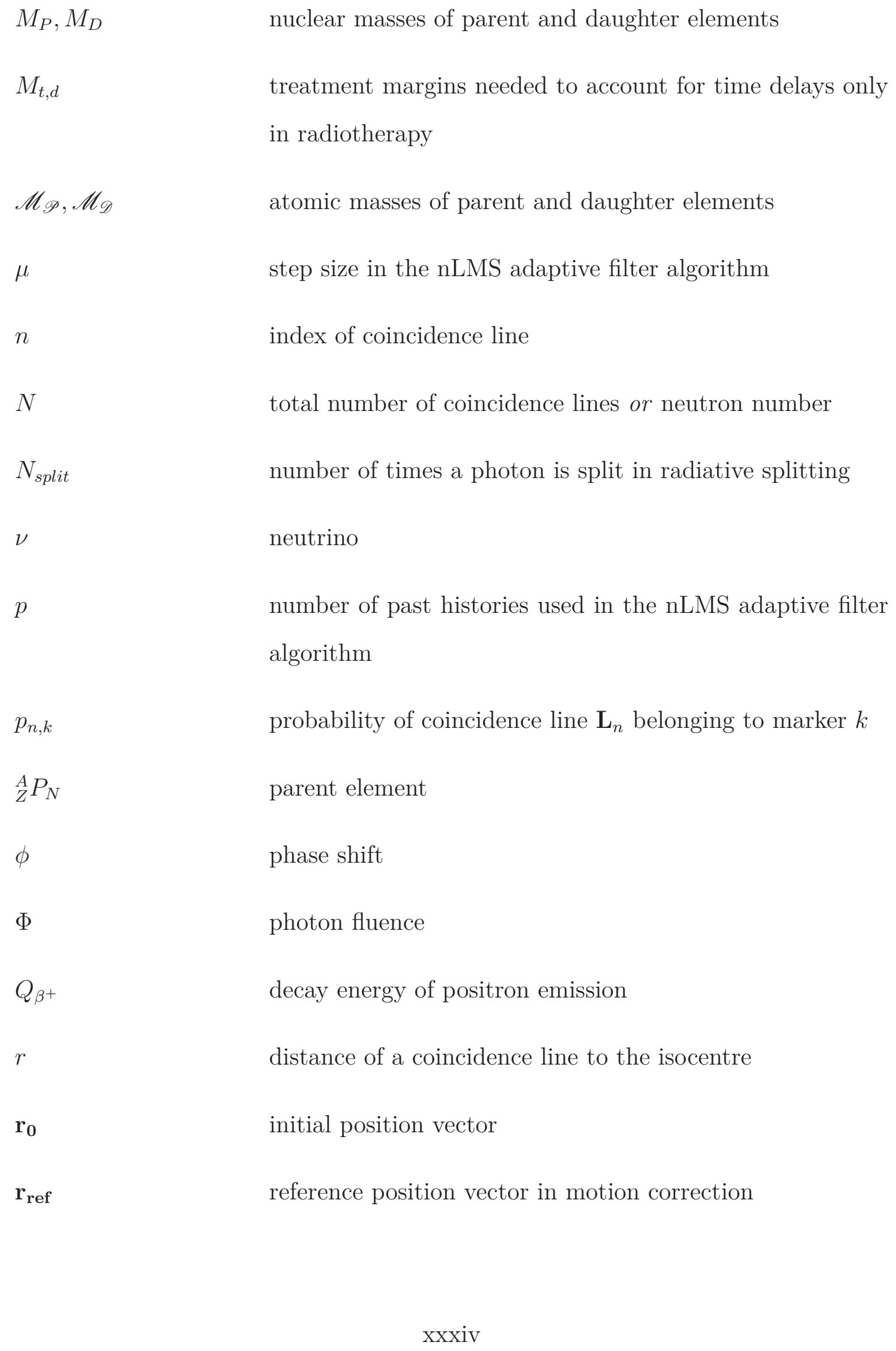

reference position vector in motion correction

number of times a photon is split in radiative splitting neutrino

number of past histories used in the nLMS adaptive filter algorithm

probability of coincidence line $\mathbf{L}_{n}$ belonging to marker $k$

parent element

phase shift

photon fluence

decay energy of positron emission

distance of a coincidence line to the isocentre

initial position vector 


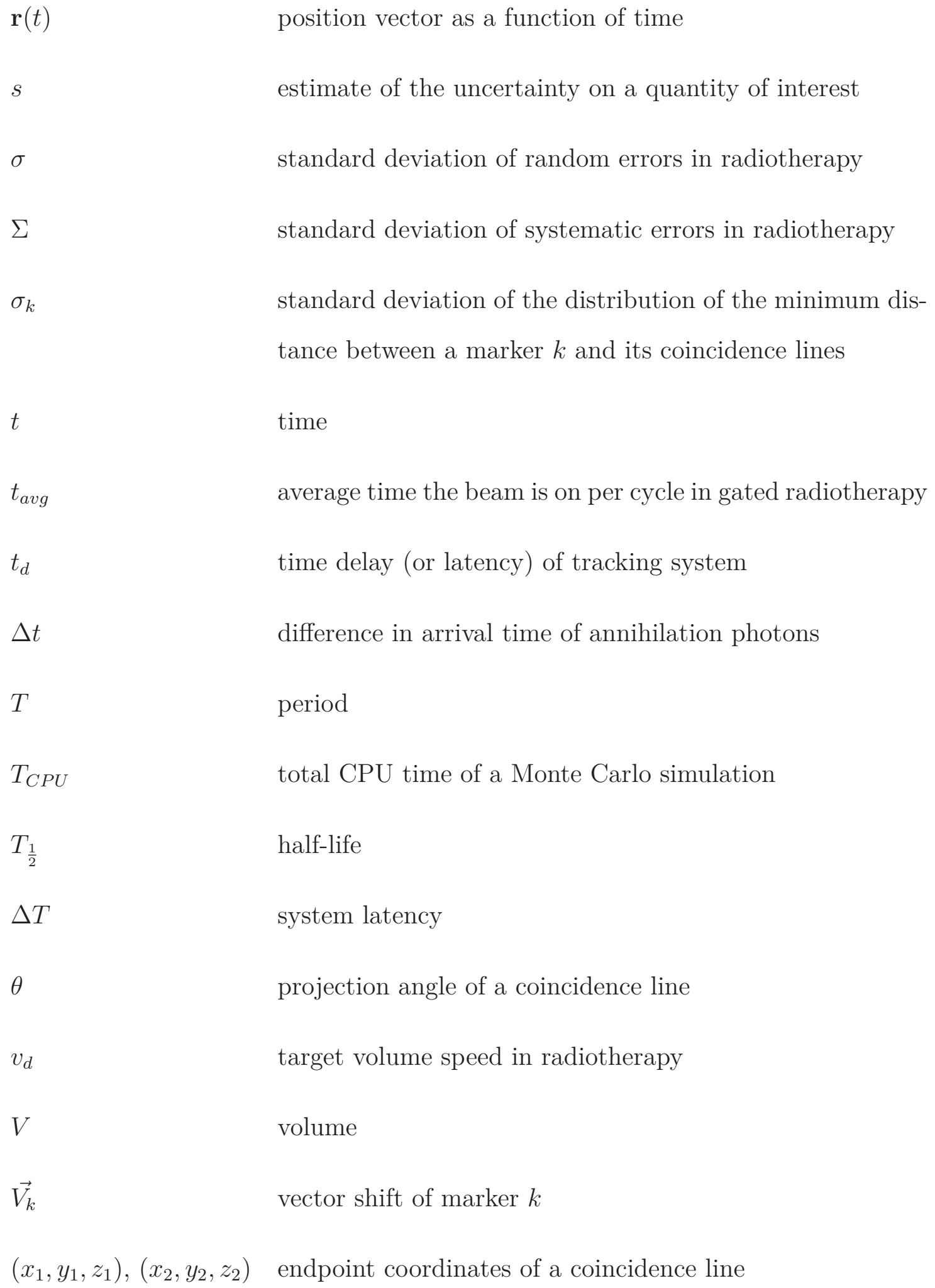




\begin{tabular}{|c|c|}
\hline$\left(x_{d}, y_{d}, z_{d}\right)$ & $\begin{array}{l}\text { coordinates of the centre of a detector element at the av- } \\
\text { erage depth of interaction }\end{array}$ \\
\hline$x(n)$ & location of marker at time $t_{n}$ \\
\hline $\mathbf{x}(n)$ & vector containing $p$ number of past marker locations \\
\hline$y_{0}$ & initial offset \\
\hline$y(n)$ & predicted location of marker at time $t_{n}$ \\
\hline$y(t)$ & position as a function of time \\
\hline$Z_{1}, Z_{2}$ & axial detector element indices \\
\hline$Z$ & atomic number \\
\hline
\end{tabular}




\section{Chapter 1}

\section{Introduction}

\subsection{Respiratory motion in radiation therapy}

The Canadian Cancer Society reports that two in five Canadians will develop cancer in their lifetime and one in four will die of cancer. [5] The majority of new cancers being diagnosed are breast, lung, colorectal, and prostate cancer. In addition, the use of radiation therapy to treat those cancers has steadily increased in Ontario between 1997 and 2007. [6] For instance, more than 30\% of lung cancer patients received radiotherapy in their first year of treatment.

Consequently, accurate delivery of radiotherapy treatments is highly desirable, especially for abdominal and thoracic tumours which are surrounded by numerous organs at risk. The accuracy of the delivered dose distribution is unfortunately limited by several factors such as patient setup, tumour delineation, and respiratory and organ motion. [7] During respiration, the thickness of a patient's lower abdominal region may change by up to $4 \%$ due to quiet breathing in the anterior-posterior (AP) direction. This can lead to errors of up to $2 \%$ in the delivered dose. [8] The tumour itself may move by up to $60 \mathrm{~mm}$ in the inferior-superior (IS) direction due to patient breathing. [9-12] This may cause the tumour to receive a lower dose than prescribed or the surrounding normal tissues to be exposed to larger doses. The tumour control 
probability $^{1}$ for lung tumours may drop from $42.4 \%$, without motion, to $37.5 \%$, if the tumour has an amplitude of motion of $10 \mathrm{~mm}$. [13] This motivates the need for motion management techniques, i.e. methods that aim to reduce the dose to normal tissues and increase the dose to the tumour when motion of the target is observed or suspected. In fact, the American Association of Physicists in Medicine (AAPM) Task Group 76 specifically recommends the use of respiratory motion management if the tumour is observed to move by more than $5 \mathrm{~mm}$ in any direction or if significant normal tissue sparing is gained through motion management. [14] Several motion management methods are in use clinically today, such as patient immobilization, breath hold, abdominal compression, respiratory gating, and tumour tracking. [14,15] Some of those methods are not always easy for the patients to comply with (breath hold) or are uncomfortable (abdominal compression). Respiratory gating only delivers radiation during certain phases of the breathing cycle or when the target is within a predetermined window. Robotic devices such as the CyberKnife (Accuray, Inc., Sunnyvale, CA) can dynamically reposition the therapy beam to follow the tumour. Both gating and beam repositioning require precise knowledge of the motion of the target.

\subsection{Patient body motion in positron emission to- mography imaging}

Positron emission tomography (PET) imaging provides in vivo information on physiological processes in the body. It is commonly used in cancer diagnosis and staging and in neurological imaging. [16] A radioactive tracer is initially injected intravenously in the patient. The tracer is a compound that has been tagged with radioactive molecules

\footnotetext{
${ }^{1}$ Tumour control probability is the probability that no clonogenic cells survive the radiotherapy treatment.
} 
and is selected based on the physiological process that needs to be observed. The goal of PET imaging is to reconstruct the internal physiological tracer distribution from detection of its decay products. For example, ${ }^{18} \mathrm{~F}$-fluorodeoxyglucose $\left({ }^{18} \mathrm{FDG}\right)$ is used as an analog to glucose. It is used in cancer diagnosis because tumour cells divide rapidly and have a high glucose metabolic activity. Tumours take up a large fraction of the radioactive ${ }^{18} \mathrm{FDG}$ and show up as hot spots on the PET images. PET imaging is also used in cardiac imaging to determine blood flow to the walls of the heart (myocardial perfusion imaging; MPI). MPI can be a good indicator of coronary artery disease (CAD) and coronary vascular disease (CVD). [17-20] Cardiac PET imaging to detect CAD is increasingly important due to the expected rise of CAD and CVD incidence in the Canadian population. [21]

PET scans have long acquisition times, from several minutes up to one hour. The length of the scans make PET scans particularly susceptible to patient body motion. In PET imaging, movement during acquisition leads to blurring and attenuation artefacts. This can cause an underestimation of the tracer activity and an overestimation of the size of the lesion or tumour. [22] Patient body motion is common in cardiac PET imaging: Klein et al. [23] reported that more than 50\% of 472 cardiac patient studies at the University of Ottawa Heart Institute exhibited mild (3 to $7 \mathrm{~mm}$ ) to severe $(>7 \mathrm{~mm})$ motion. The motion may be voluntary or involuntary. Voluntary movement is usually unpredictable, for example if the patient moves because they feel uncomfortable. Involuntary movement includes mostly regular motion such as breathing and the beating of the heart.

Respiratory and cardiac gated PET imaging is the most common method of acquiring motion compensated PET images. [24] Gated PET imaging consists in synchronizing the data acquisition with a description of the lesion or organ movement. As in the case of respiratory motion in radiotherapy, this requires a method to track the motion in question. 


\subsection{PeTrack}

In both radiation therapy and PET imaging, there is a need to detect or monitor patient body motion and compensate for it. To that end, a three-dimensional (3D) tracking system which uses fiducial positron emission markers was previously developed. [25] The technique is called PeTrack, for Positron Emission Tracking. PeTrack can track fiducial positron emission markers in 3D. Its tracking performance was evaluated before, but its direct use for motion compensated radiotherapy or PET imaging has never been assessed. [1]

\subsection{Thesis overview}

This thesis will present various applications of PeTrack in radiotherapy and PET imaging. The goal is to show the potential for PeTrack to address the issue of patient body motion. Chapter 2 will provide an overview of basic positron physics and of the PeTrack system. Chapter 3 will cover the application of PeTrack for respiratory-gated radiotherapy. Chapter 4 will present the modifications to the tracking algorithm in order to use PeTrack during PET imaging. Chapter 5 will demonstrate two proofs of concept applications of PeTrack in cardiac PET imaging: respiratory-gated PET imaging using PeTrack and event-by-event motion correction using PeTrack. Finally, Chapter 6 discusses summarizes the current work and discusses future work. 


\section{Chapter 2}

\section{Overview of PeTrack}

This chapter provides an overview of PeTrack. The physics of positron emission and coincidence is reviewed. The prototype PeTrack detectors and tracking algorithm are described. The chapter concludes with a summary of the performance of the system. Some of the background material in this chapter has been presented previously in my master's degree thesis. [2]

\section{$2.1 \quad$ Positron emission physics}

\subsubsection{Positron emission}

In positron emission, also known as $\beta^{+}$decay, a proton decays into a neutron. A positron $e^{+}$and a neutrino $\nu$ are produced and emitted from the nucleus in the process. This decay can be written as:

$$
{ }_{Z}^{A} P_{N} \rightarrow{ }_{Z-1}^{A} D_{N+1}+e^{+}+\nu,
$$


where ${ }_{Z}^{A} P_{N}$ is the parent atom (with mass number $A$, atomic number $Z$, and neutron number $N$ ) and ${ }_{Z-1}^{A} D_{N+1}$ is the daughter nucleus. The energy of the decay is:

$$
Q_{\beta_{+}}=\left[M_{P}-\left(M_{D}+m_{e}\right)\right] c^{2}
$$

where $M_{P}$ and $M_{D}$ are respectively the nuclear mass of the parent and daughter atoms, $m_{e}$ is the mass of the positron, and $c$ is the speed of light in vacuum. The decay energy can be rewritten in terms of the atomic masses of the parent and daughter atoms, $\mathscr{M}_{P}$ and $\mathscr{M}_{D}$, by adding and subtracting $Z m_{e}$ from the right hand side:

$$
\begin{aligned}
Q_{\beta_{+}} & =\left[M_{P}+Z m_{e}-\left(M_{D}+Z m_{e}+m_{e}\right)\right] c^{2} \\
& =\left[\mathscr{M}_{P}-\left(\mathscr{M}_{D}+2 m_{e}\right)\right] c^{2}
\end{aligned}
$$

The above illustrates that positron decay can only occur if the atomic mass of the parent exceeds the atomic mass of the daughter by at least twice the rest mass energy of a positron (i.e. $1.022 \mathrm{MeV}$ ). The emitted neutrino carries away some of the energy of the decay. Thus the emitted positrons have a continuous spectrum of energy. The maximum kinetic energy of an emitted positron is equal to the energy of the decay process, ignoring the recoil energy of the daughter nucleus. In the field of medical physics, the average energy of the positron energy spectrum is customarily approximated as $\frac{1}{3}$ of the maximum kinetic energy. [26]

\subsubsection{Positron annihilation}

At low energy, positrons lose most of their energy by ionization. Eventually, the positron annihilates with an electron; the process produces two annihilation gamma rays of energy $511 \mathrm{keV}$ each:

$$
e^{+}+e^{-} \rightarrow \gamma+\gamma
$$


Annihilation usually occurs when the positron and electron are close to rest. By conservation of linear momentum, the annihilation photons are emitted back-to-back, i.e. at $180^{\circ}$ in their centre-of-momentum frame.

\subsubsection{Scintillation detector}

Scintillation detectors are commonly used to detect annihilation photons. A scintillator (typically an inorganic crystal) absorbs an annihilation photon and re-emits some of the energy in the form of visible light. A photo-multiplier tube coupled to the crystal collects the light and produces an electric signal.

\subsubsection{Coincidence detection}

Coincidence detection forms the basis of PET imaging. A coincidence event corresponds to the simultaneous detection of annihilation photons in opposite detectors. In practice, there is a finite time window within which two detected events will be considered in coincidence. This is called the coincidence time window. The time window in PET imaging can range from 4 to 12 ns. [27] For true coincidence events, the geometric line which connects the centre of the two detector elements which recorded the event is called a line of response (LOR). Sometimes, the concept of tube of response (TOR) is useful. A TOR is the 3D tube that joins the surface of two detector elements. $^{1}$

Coincidence events can be sorted into three different types: true, scattered, and random coincidences (see Figure 2.1). A true coincidence corresponds to two backto-back photons created in the same annihilation event detected in opposite detectors within the coincidence time window. The location of the annihilation is somewhere along the LOR. In a scattered coincidence, one or both of the annihilation photons are

\footnotetext{
${ }^{1}$ Tube is somewhat of a misnomer since most detector elements in PET have rectangular surfaces.
} 
deflected from their original trajectory by scattering. In a random coincidence, two photons from two different annihilation events are detected within the coincidence time window and misregistered as a coincidence. The location of the annihilation event does not typically fall along the LOR of scattered and random coincidences.

\subsubsection{Positron range and photon non-collinearity}

Two effects can lead to errors when attempting to locate the location of a positron emitting nucleus. First, when a positron is emitted from a nucleus, it travels a certain distance (called the positron range) before annihilating with an electron. This means that the nucleus is not generally found along the LOR. Positron range depends on the kinetic energy of the positrons and on the medium in which it travels. As a rough approximation of positron range, the CSDA range ${ }^{2}$ of $182 \mathrm{keV}$ positrons in water is approximately $^{3} 0.36 \mathrm{~mm}$. Second, residual momentum in the positron-electron pair before annihilation causes the photons to be emitted at an angle smaller than $180^{\circ}$. This effect is called photon non-collinearity. The distribution of the deviation angle in the human body from ${ }^{18} \mathrm{FDG}$ can be modelled as a Gaussian function centered at $0^{\circ}$ and with FWHM of $0.54^{\circ}$. [28] If the distance between the detectors is $80 \mathrm{~cm}$, the non-collinearity of photons results in a positional uncertainty of $1.9 \mathrm{~mm}$.

\subsubsection{Time of flight}

If the time of detection of each photon in a coincidence event can be measured precisely, then the time of flight (TOF) method can determine the location of the annihilation event along the LOR (see Figure 2.3). Assume an annihilation event occurs along the LOR a distance $d$ away from the centre of the LOR. Let the difference in

\footnotetext{
${ }^{2}$ Continuous Slowing Down Approximation

${ }^{3}$ This is the average energy of positrons emitted by ${ }^{22} \mathrm{Na}$.
} 


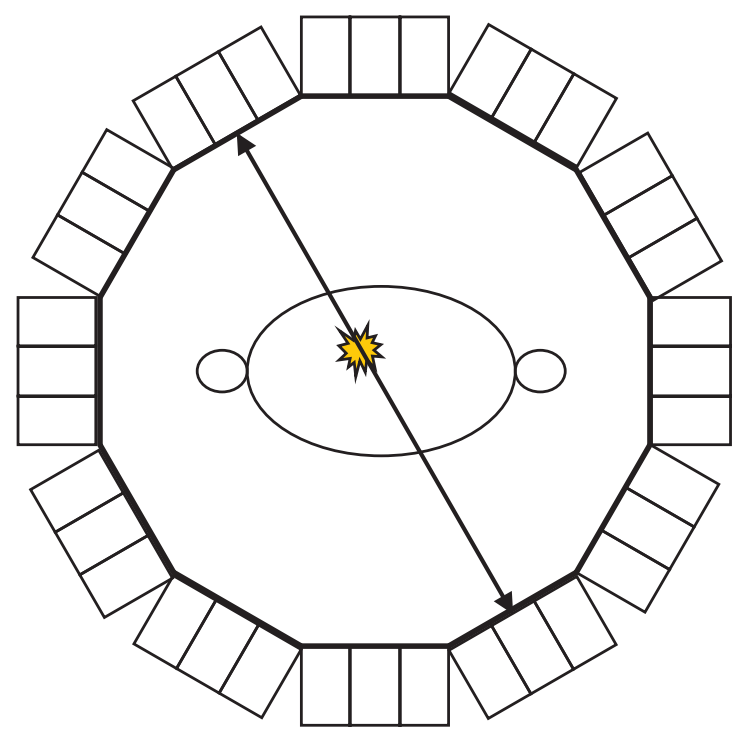

(a) True coincidence

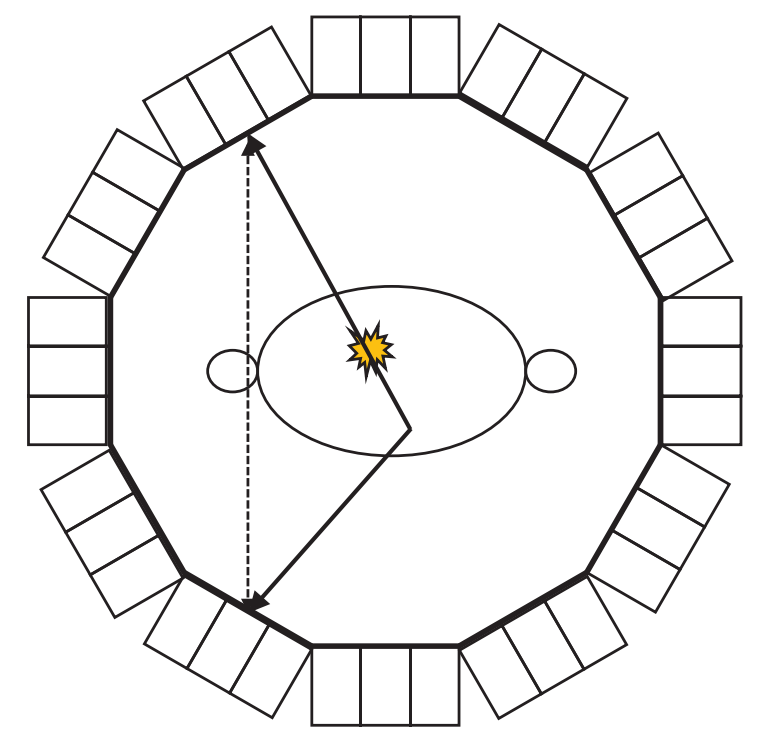

(b) Scattered coincidence

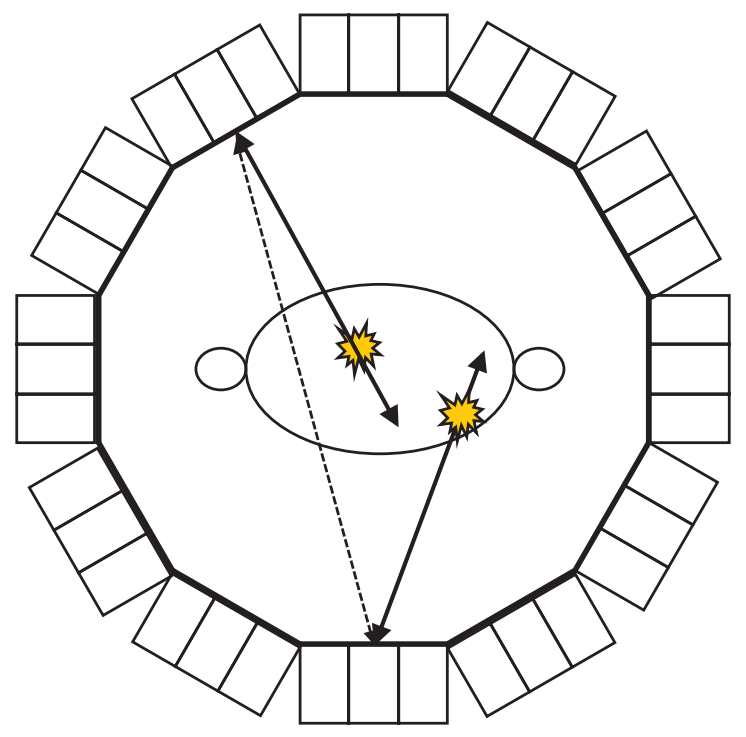

(c) Random coincidence

Figure 2.1: Illustration of the different types of coincidences. a) True coincidence: two back-to-back photons created in the same annihilation event are detected in opposite detectors within the coincidence time window. b) Scattered coincidence: one or both of the photons are deflected due to scattering before being detected. c) Two photons from two different annihilation events are detected within the coincidence time window. The dashed lines in b) and c) represent the mispositioned LOR. 


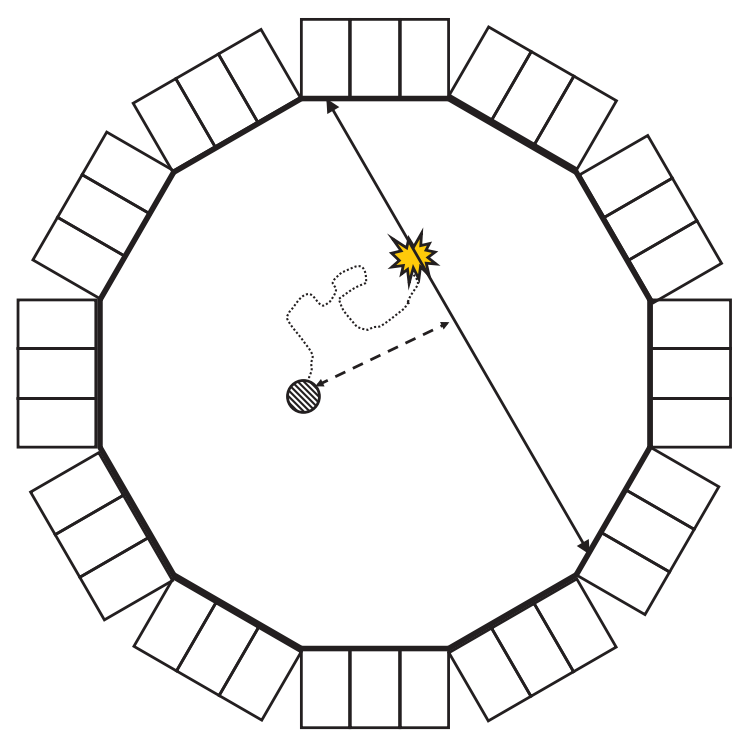

(a) Positron range

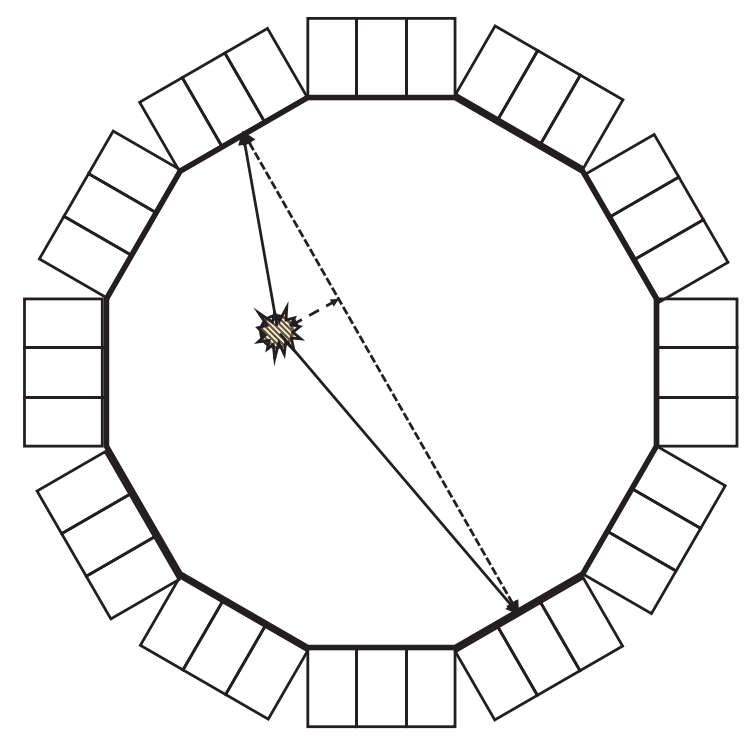

(b) Photon non-collinearity

Figure 2.2: Illustration of the errors (long dashed line) introduced by positron range (a) and photon non-collinearity (b) in the determination of the location of the positron emitting nucleus (dashed circle).

the detection time of the two photons be $\Delta t$. The distance $d$ is given by:

$$
d=\frac{\Delta t \times c}{2}
$$

The current generation of commercial TOF PET scanners has a timing resolution in the range 500 to $600 \mathrm{ps}$ (7.5 to $9.0 \mathrm{~cm}$ positional resolution). [29-31]

\subsubsection{PET scanner}

PET scanners are most commonly arranged in a block ring configuration (see Figure 2.4). The axial direction runs along the direction of the patient bed. The plane perpendicular to the axial direction is called the transaxial (or transverse) plane. Detector elements are arranged in arrays (transaxial×axial) called blocks. Sometimes, detector blocks are also arranged in arrays called modules. A detector element in the transaxial plane can be in coincidence with a fan of opposite detector elements (see 


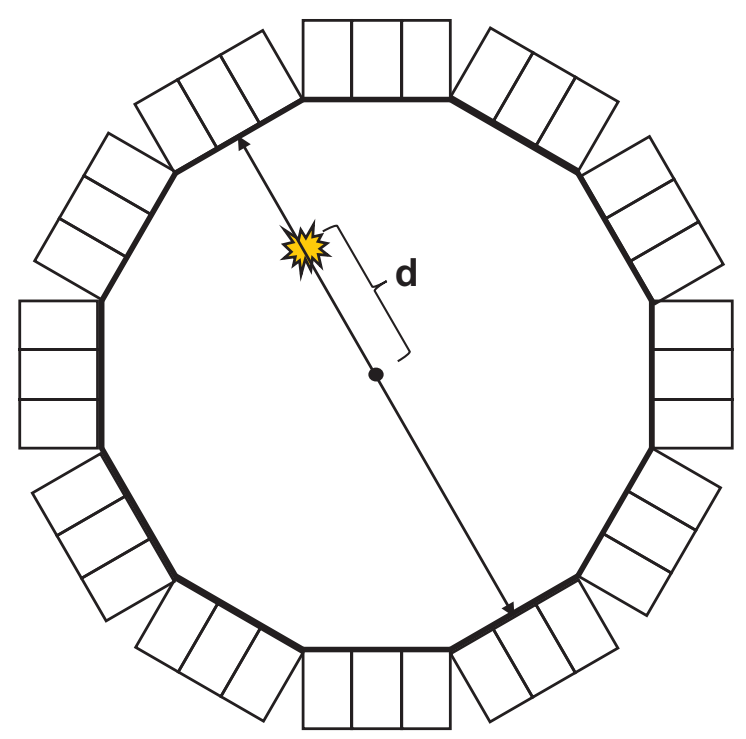

Figure 2.3: Illustration of principle of time of flight in coincidence detection. The distance $d$ is from the location of the annihilation event to the centre of the LOR. The path length difference between the two photons is $2 d$. If the difference in the time of detection of each photon is $\Delta t$, then the path length difference is given by $2 d=\Delta t \times c$ where $c$ is the speed of light in air.

Figure 2.4(a)). Coincidences can be between detector elements in the same transaxial plane (direct) or between detector elements in two different transaxial planes (oblique) (see Figure 2.4(b)).

\subsubsection{Coincidence data storage}

Coincidence events recorded in PET are typically stored in one of two formats: sinogram or list mode format. If only coincidences within the same ring of detector elements are allowed (which is known as 2D PET), the LORs are binned into a two dimensional (2D) matrix. Each element of the matrix corresponds to the number of events detected by a specific detector element pair. The matrix is organized such that each row represents the projection of the tracer activity at a particular projection angle $\theta$. Each column represents the radial offset, $r$, from the centre of the scanner (see 


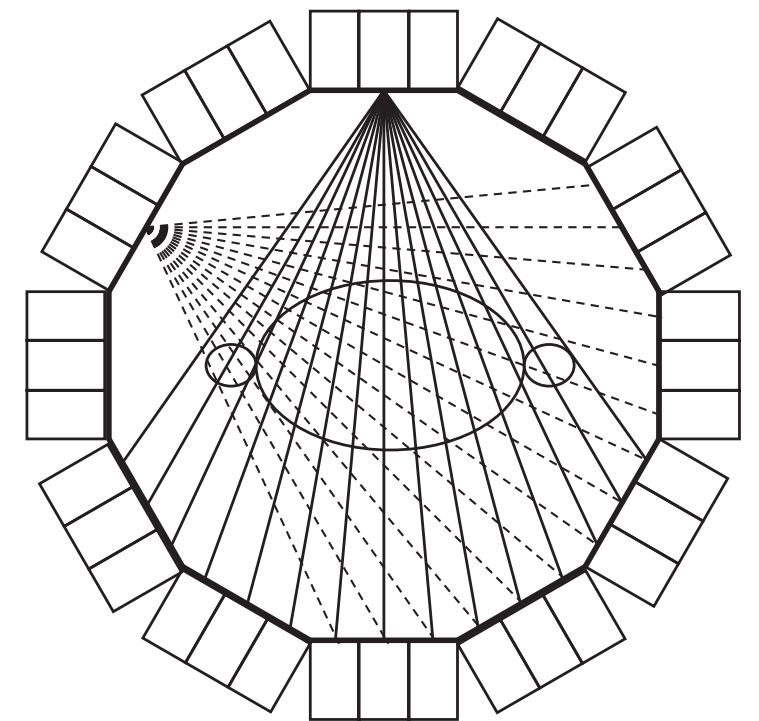

(a) Transaxial (or transverse) slice of a block ring scanner.

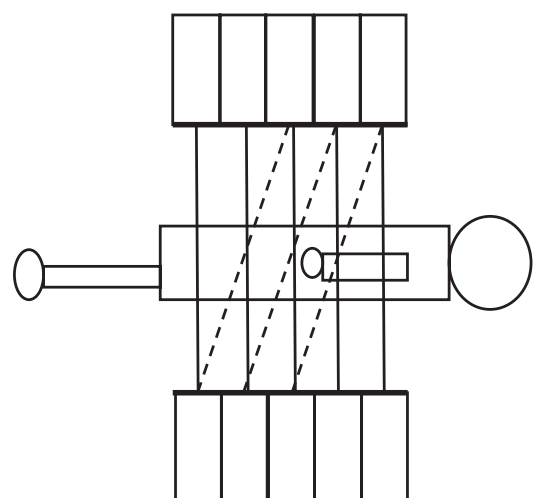

(b) Axial slice of a block ring scanner.

Figure 2.4: The most common PET scanner configuration is the block ring configuration. Detector elements are arranged in arrays called blocks. (a) In the transaxial (or transverse) plane of the scanner, the blocks are distributed around the gantry in a regular polygon pattern. A detector element can be in coincidence with a fan of opposite detector elements. (b) In the axial plane of the scanner, detector elements can accept coincidences that are oblique (dashed line) as well as direct (solid line). 
Figure 2.5). Each element $(r, \theta)$ in the matrix can record activity originating from location $(x, y)$ according to the relationship:

$$
r=x \cos \theta+y \sin \theta
$$

The $2 \mathrm{D}$ matrix is called a sinogram because activity at location $(x, y)$ will trace a sinusoidal path in the matrix, as by the previous equation.

Most scanners today operate in 3D mode. In 3D PET, coincidences between detector elements belonging to different rings are allowed. The data can be stored in a 3D matrix which incorporates the axial coordinate of the 2D sinograms. However, the details of 3D PET data storage are outside the scope of this work.

In list mode format, each coincidence event is stored individually. Information on the LOR assigned to each event (e.g. the indices of the pair of detector elements which detected the event) and its time of detection is recorded. 


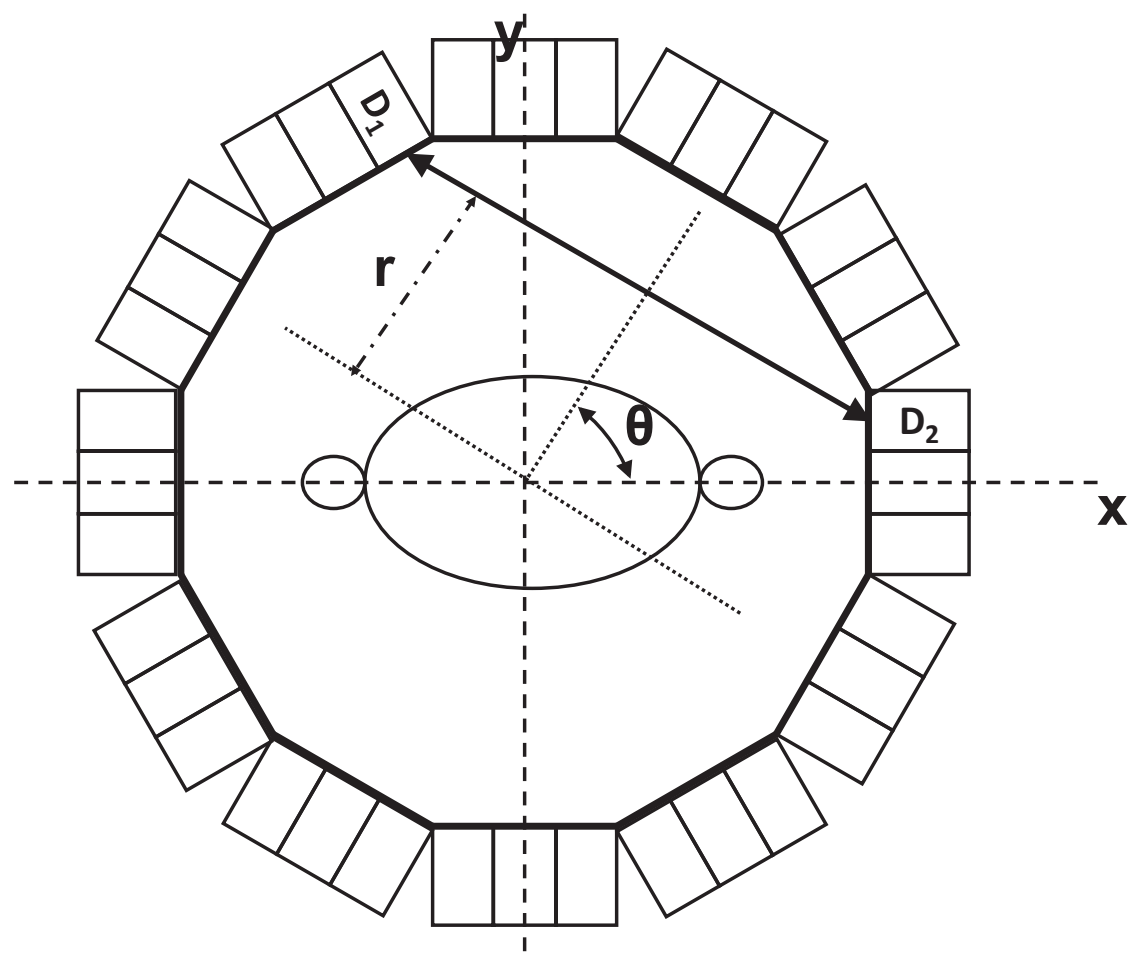

Figure 2.5: Geometric definition of the angular and radial bins in sinograms. Two coincidence photons detected by detector elements $\mathbf{D}_{\mathbf{1}}$ and $\mathbf{D}_{\mathbf{2}}$ are assigned a LOR defined by $r$ and $\theta$. 


\subsection{PeTrack}

PeTrack is a system that can track the 3D location of point-like positron emission fiducial markers. At its core is an expectation-maximization (EM) clustering algorithm. The system also consists of position-sensitive gamma ray detectors that record coincidence events from positron annihilation. Sections 2.2.1 and 2.2.2 are based on work by $\mathrm{Xu}$ et al. [25]. Sections 2.2.5 and 2.2.6 are an extension of the work I conducted during my master's degree [2] and were published in [1]. Section 2.2.9 presents results from Mojgan Soleimani's master's thesis on characterizing a pre-prototype version of the PeTrack detectors. [3] Section 2.2.11 presents results from Simin Razavi's master's thesis, which explored the sensitivity and calibration of the PeTrack prototype detector system. [32].

\subsubsection{Principle of operation}

It is possible to determine the location of a point-like positron emitting fiducial marker using LORs. ${ }^{4}$ The position of the marker is the point in $3 \mathrm{D}$ space that minimizes the root mean square (RMS) distance to the LORs. This principle can be extended to multiple markers as long as each LOR is correctly assigned to the marker that produced it. The location of each marker minimizes the RMS distance to their LOR. PeTrack uses this concept to achieve real time tracking of multiple low activity fiducial positron emission markers.

\subsubsection{Multimarker localization algorithm}

PeTrack uses an expectation-maximization clustering algorithm to track the location of $K$ markers. LOR collected during a certain sampling interval, called the localization

\footnotetext{
${ }^{4}$ In this context, LOR refers to the individual coincidence event and is not limited to a line in space that connects two detector elements.
} 
interval, are clustered into $K$ groups belonging to each of the $K$ markers. Each marker is assigned a marker index $k$ which runs from $k=1, \ldots, K$. The distance from the centre of a marker to its LOR is assumed to follow a Gaussian distribution with a mean of zero and a standard deviation of $\sigma_{k}$. The probability density function (PDF) for this Gaussian distribution is given by:

$$
G\left(\left|\vec{d}\left(\mathbf{L}_{n}, \vec{m}_{k}\right)\right|, \sigma_{k}\right)=\frac{1}{\sqrt{2 \pi} \sigma_{k}} \exp \left(-\frac{\left|\vec{d}\left(\mathbf{L}_{n}, \vec{m}_{k}\right)\right|^{2}}{2 \sigma_{k}^{2}}\right)
$$

In this equation, $k$ is the marker index and $\vec{d}\left(\mathbf{L}_{n}, \vec{m}_{k}\right)$ is the minimum distance vector that connects an estimated marker location $m_{k}$ to one of the LOR $\mathbf{L}_{n}$ associated with this marker. The PDF $G\left(\left|\vec{d}\left(\mathbf{L}_{n}, \vec{m}_{k}\right)\right|, \sigma_{k}\right)$ is not a two-dimensional function. It is a one-dimensional function of the variable $\vec{d}\left(\mathbf{L}_{n}, \vec{m}_{k}\right)$, but the standard deviation, $\sigma_{k}$, is updated throughout the iterations of the algorithm.

The algorithm starts at iteration index $i=0$ by making an initial estimate of the location of the markers (see Section 2.2.3). The initial standard deviation is set to $\sigma_{k}=5 \mathrm{~mm}$ and the relative activity of each marker is set to $\alpha_{k}=\frac{1}{K}$. Although the real relative activity of the markers is known a priori, having $\alpha_{k}=\frac{1}{K}$ be a parameter that is updated every iteration allows more flexibility in the soft clustering scheme of the algorithm. The following two steps are performed until the position of each marker changes by less than the convergence criterion of $0.05 \mathrm{~mm}$ :

1. Expectation step: the probabilities $p_{n, k}^{(i)}$ of each $\operatorname{LOR} \mathbf{L}_{n}, n=1, \ldots, N$ (where $N$ is the total number of LORs used by the algorithm) belonging to each cluster $k=1, \ldots, K$ are calculated as follows:

$$
p_{n, k}^{(i)}=\frac{\alpha_{k}^{(i)} G\left(\left|\vec{d}\left(\mathbf{L}_{n}, \vec{m}_{k}^{(i)}\right)\right|, \sigma_{k}^{(i)}\right)}{\sum_{j=1}^{K} \alpha_{j}^{(i)} G\left(\left|\vec{d}\left(\mathbf{L}_{n}, \vec{m}_{j}^{(i)}\right)\right|, \sigma_{j}^{(i)}\right)}
$$

Each LOR $\mathbf{L}_{n}$ is partly assigned to every marker with probability $p_{n, k}^{(i)}$. 
2. Maximization step: the relative activity of each marker, $\alpha_{k}^{(i)}$, and the standard deviation of the minimum distance between the LOR and their corresponding markers, $\sigma_{k}^{(i)}$, are updated according to the probabilities obtained from the expectation step. The new parameters are given by:

$$
\begin{gathered}
\alpha_{k}^{(i+1)}=\frac{\sum_{n=1}^{N} p_{n, k}^{(i)}}{N} ; \\
\sigma_{k}^{(i+1)}=\sqrt{\frac{\sum_{n=1}^{N} p_{n, k}^{(i)} \cdot\left|\vec{d}\left(\mathbf{L}_{n}, \vec{m}_{k}^{(i)}\right)\right|^{2}}{\sum_{n=1}^{N} p_{n, k}^{(i)}}} .
\end{gathered}
$$

The necessary vector shift to minimize the RMS distance of a marker to its LOR is calculated for each previously estimated marker position. The vector shift is the result of the weighted sum of all $\vec{d}\left(\mathbf{L}_{n}, \vec{m}_{k}^{(i)}\right)$, with each weight given by Equation (2.8):

$$
\vec{V}_{k}^{(i)}=\frac{\sum_{n=1}^{N} p_{n, k}^{(i)} \cdot \vec{d}\left(\mathbf{L}_{n}, \vec{m}_{k}^{(i)}\right)}{\sum_{n=1}^{N} p_{n, k}^{(i)}} .
$$

The updated estimated position of the marker is thus:

$$
\vec{m}_{k}^{(i+1)}=\vec{m}_{k}^{(i)}+\vec{V}_{k}^{(i)}
$$

Some LOR may correspond to scattered or random coincidences instead of true coincidences. Using those LOR in the localization of the markers would introduce errors because these corrupted LOR contain no useful spatial information about the location of the marker. To prevent such errors, the probability of a LOR $\mathbf{L}_{n}$ belonging to marker $k$ is set to zero for marker $k$ if the distance between the LOR $\mathbf{L}_{n}$ and the estimated location of the marker $\vec{m}_{k}^{(i)}$ is larger than twice the standard deviation of the cluster of LOR: $\left|\vec{d}\left(\mathbf{L}_{n}, \vec{m}_{k}^{(i)}\right)\right|>2 \sigma_{k}^{(i)}$. Figure 2.6 illustrates the logical flow of the algorithm. 


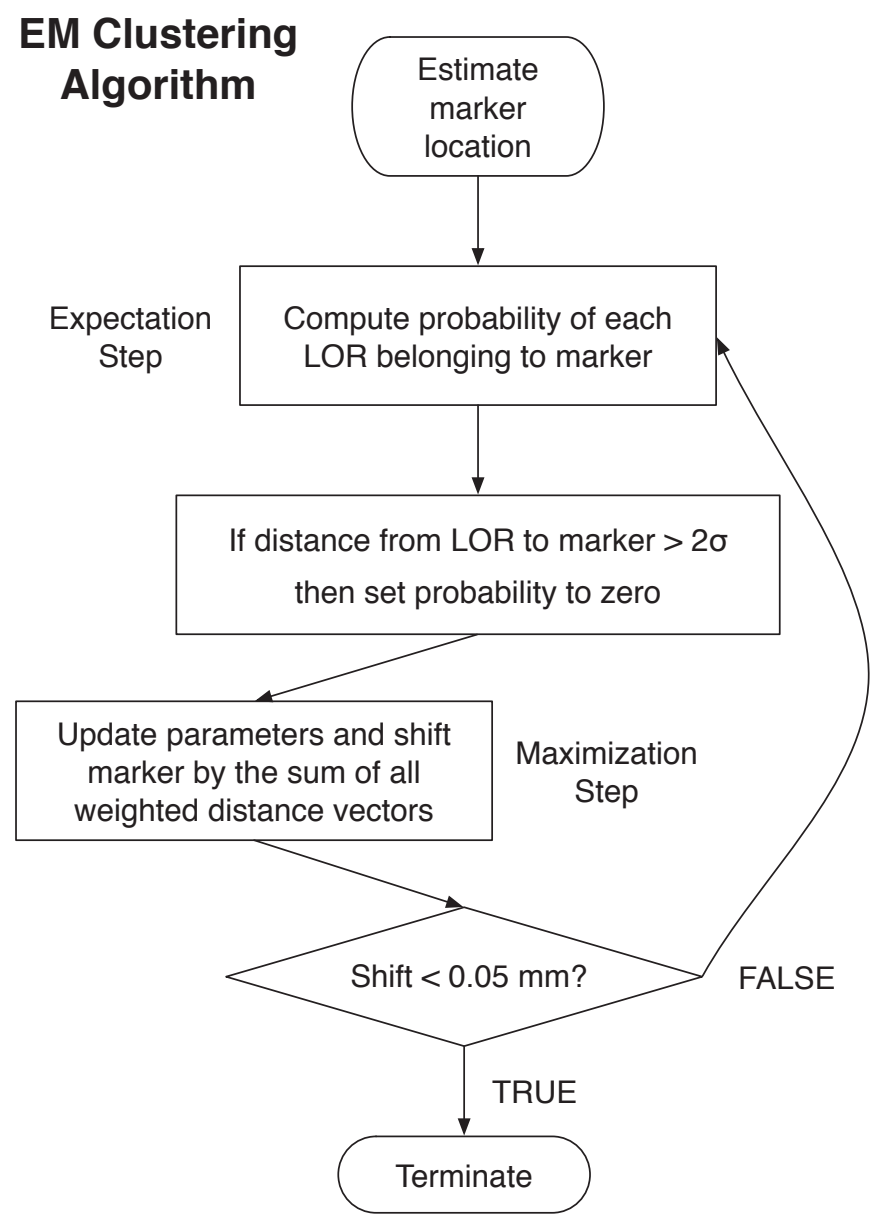

Figure 2.6: Logical flow of the expectation-maximization algorithm. Modified from [2]. 


\subsubsection{Initialization of the algorithm}

The location of the markers at the start of the first localization interval is estimated using a clustering technique proposed by Gundogdu. [33] The starting points of the algorithm are randomly selected around the centre of mass of the first set of collected LOR. The centre of mass is found by using midpoints. A midpoint is defined as the point of intersection of two LOR, if they intersect, or the halfway point on the minimum distance vector that joins two LOR, if they do not intersect. Since the RMS distance between LOR should be minimized at a marker location, the density of midpoints is expected to be higher in the vicinity of a marker. The initialization proceeds as follows:

1. Randomly select three LOR from the first set of events recorded. Typically, 300 LOR per localization are collected for three markers.

2. Calculate the minimum distance between each pair of LOR. If all three LOR are within $5 \mathrm{~mm}$ of each other, it is highly probable that they originated from the same marker; otherwise, repeat step 1.

3. Calculate the three midpoints between each pair of LOR.

4. Repeat 500 times steps 1 through 3.

5. Take the centre of mass of the 1500 midpoints obtained from steps 1 through 4 .

6. Initialize the expectation-maximization algorithm at a random location around the centre of mass for each marker. The maximum possible distance from the centre of mass is set to the average distance between the markers.

This procedure is done once at the beginning of the tracking. For each subsequent localization, the last known location of the markers is used as the initial estimate of their position. 


\subsubsection{Fail-safe methods}

The initialization contains a random component, thus it is not guaranteed to correctly locate the markers on the first try. In order to identify failed localizations and to locate the markers correctly, fail-safe methods are required. Failed localizations can be identified by any of the following conditions, using a 3-marker scenario as an example:

- Condition 1: All three markers are assigned to the same cluster of LOR.

- Condition 2: Two markers are assigned to the same cluster of LOR.

- Condition 3: An unusually high number of LOR are assigned to a single marker or the standard deviation of the distribution of the minimum distance between the LOR and their respective marker is unusually high. For three markers of equal activity, approximately one third of the total number of LOR detected during the localization time interval is expected to be assigned to each marker. If more than half the total number of LOR is assigned to a marker, the localization has failed. The standard deviation is considered abnormally high when it is larger than $5 \mathrm{~mm}$. The average value of the standard deviation was determined to be $3.2 \pm 0.3 \mathrm{~mm}$ from Monte Carlo and phantom studies.

Three fail-safe methods are implemented to address each of the conditions above:

- Method 1: If all three markers were assigned to the same cluster (condition 1), this signifies that one marker was successfully located, while the other two markers were wrongly assigned to the same location as the successful marker. In this case, the two failed markers are re-initialized at a set distance around the successful marker. The re-initialization distance should be on the order of the distance between the true locations of the markers. 
- Method 2: If only two markers were assigned to the same cluster (condition 2), this means that two markers were successfully located while the third marker was incorrectly assigned to the same location as one of the two successful markers. In this case, the failed marker is re-initialized at a set distance around the half-way point of the distance vector that joins the two successful markers and on the perpendicular plane to that distance vector. The distance should be of the order of the distance between the true locations of the markers.

- Method 3: If condition 3 is met, the failed marker is re-initialized randomly around its current location.

Once the location of the failed markers has been re-initialized using the appropriate method, the algorithm will re-attempt localization of the markers. After three attempts, if the algorithm still fails to locate the markers, it will move on to the next set of collected LORs. If a marker fails to be located five consecutive times, its location is re-initialized randomly around the mean midpoint of the current set of LORs. The time from the onset of the tracking until all markers are correctly located is called the initialization phase. Though those fail-safe methods were initially designed for three markers, they can be adapted to account for more or fewer than three markers. For the special case of tracking a single marker (which is used in the subsequent chapters), a failed localization causes the standard deviation to be too large $(>5 \mathrm{~mm})$; only Method \#3 above is applied in this case.

\subsubsection{Linear prediction}

A potential application of PeTrack is for real time tumour tracking during radiotherapy. Ideally, the therapy beam would follow the tumour motion in real time. Unfortunately, inherent latency in electronic, computer, and mechanical systems prevent the treatment from being perfectly synchronized with the tracking of the tumour. 
There are delays due, for example, to the computational time required to locate the tumour and to the mechanical reaction time of the hardware. Litzenberg et al. report a delay of $55 \mathrm{~ms}$ in a dynamic multi-leaf collimator (MLC) system. [34] The latency of the Elekta Agility MLC system for tumour tracking varies between 37 and $69 \mathrm{~ms}$. [35] Seppenwoolde et al. have a $90 \mathrm{~ms}$ delay in a beam-gated radiotherapy system. [36] The CyberKnife robotic treatment device has a delay of $115 \mathrm{~ms}$ in its Synchrony Respiratory Tracking System. [37] These delays justify the need for motion prediction. Given that a system with latency $\Delta T$ has located the tumour at time $t$, the tumour location at time $t+\Delta T$ needs to be predicted. The system latency should be kept to a minimum since prediction error increases with latency. [38] A linear prediction model based on the normalized least mean squares (nLMS) adaptive filter is integrated to PeTrack. This model predicts the future location of a marker based on a linear combination of its $p$ past locations. The nLMS adaptive filter was chosen for its simplicity and ease of implementation. It was also shown to perform as well or better than alternatives such as the Kalman filter when predicting patient breathing motion at various latencies. [38] The nLMS adaptive filter proceeds as follows: Let $x(n)$ be the location of the marker at time $t_{n}$ as determined by the tracking algorithm and $\mathbf{x}(n)$ be a vector of the $p$ past locations of the algorithm:

$$
\mathbf{x}(n)=[x(n), x(n-1), \ldots, x(n-p+1)]
$$

The predicted location of the marker at time $t_{n}, \mathrm{y}(\mathrm{n})$, is given by:

$$
y(n)=\boldsymbol{\alpha}(n) \cdot \mathbf{x}(n)
$$


where $\boldsymbol{\alpha}(n)$ is a vector containing the coefficients of the filter. The coefficients are initially set to zero and are updated according to:

$$
\begin{aligned}
e(n) & =x(n+1)-y(n) \\
\boldsymbol{\alpha}(n+1) & =\boldsymbol{\alpha}(n)+\frac{\mu e(n) \mathbf{x}(n)}{\|\mathbf{x}(n)\|^{2}+\Gamma}
\end{aligned}
$$

where $\mu$ is the step size of the filter and $\Gamma$ is a small positive constant. The step size controls the learning rate of the algorithm and $\Gamma$ prevents numerical instability in the denominator. For the purpose of predicting breathing motion, we require the number $p$ of past locations used in the prediction to cover at least one full breathing cycle. ${ }^{5}$ The step size is set to 1 and $\Gamma$ to $1 \times 10^{-9}$. Those values were found to minimize the prediction error over the course of the development of PeTrack. ${ }^{6}$

\subsubsection{Performance of the tracking algorithm}

The performance of the PeTrack tracking algorithm, of the linear prediction, and of the fail-safe methods were evaluated previously by Monte Carlo simulations of a prototype detector system (the prototype is described in Section 2.2.8) and phantom studies. [1] The tracking algorithm (excluding prediction) performs with submillimetre accuracy and precision when tracking markers at a frequency of $10 \mathrm{~Hz}$ using approximately 100 LORs per marker. Those LORs consisted only of events that can be recorded with the prototype detector system. The 3D root mean square error (RMSE) determined from Monte Carlo simulations is $0.44 \mathrm{~mm}$ on average. The precision (or repeatability) of the tracking in 3D is $0.49 \mathrm{~mm}$. The $3 \mathrm{D}$ prediction error determined from phantom studies is $1.3 \pm 0.6 \mathrm{~mm}$, when predicting the location of the

\footnotetext{
${ }^{5}$ For example, if the period of the breathing cycle is $4 \mathrm{~s}$ and the localization frequency is $10 \mathrm{~Hz}$, then 40 past locations are used to predict the next location of the marker.

${ }^{6}$ Unpublished results.
} 
markers $100 \mathrm{~ms}$ ahead. The automatic initialization is fast and robust. The system can also detect failed localizations.

\subsubsection{Fiducial markers}

Fiducial markers used for tracking by PeTrack should be point-like (e.g. small spherical core $0.5 \mathrm{~mm}$ or less in diameter). Encasing the core in a small tungsten, titanium, or gold capsule will limit the effect of positron range, which is much smaller in those metals than in water. If the markers are to be implanted in tissue for tumour tracking, their design can resemble brachytherapy seeds. ${ }^{7}$ The capsule would have a length of 2 to $4 \mathrm{~mm}$ and a diameter of 0.5 to $0.8 \mathrm{~mm}$. This design makes it easier to handle the capsules as opposed to a completely spherical design. If the markers are instead intended for external use (e.g. if they are placed on the abdomen of a patient), their design can be much more flexible in size or shape. The positron emission isotope can be ${ }^{124} \mathrm{I}\left(\mathrm{T}_{\frac{1}{2}}=4.2\right.$ days $),{ }^{74} \mathrm{As}\left(\mathrm{T}_{\frac{1}{2}}=17.8\right.$ days $)$, or ${ }^{84} \mathrm{Rb}\left(\mathrm{T}_{\frac{1}{2}}=32.7\right.$ days $)$ depending on the application.

\subsubsection{Prototype detector system}

A prototype PeTrack detector system was built in-house. ${ }^{8}$ The system uses position sensitive gamma ray detectors to collect LORs from fiducial positron emission markers. Unlike PET imaging, the PeTrack system does not require a full ring of detectors. Instead, two pairs of detector modules are mounted at $90^{\circ}$ on an aluminum frame (see Figure 2.7). Each module consists of a $2 \times 2$ array of detector blocks. Each detector block consists of a $12 \times 12$ pixellated array of bismuth germanate (BGO) scintillator crystals wrapped in Teflon and coupled to a position sensitive photomultiplier tube

\footnotetext{
${ }^{7}$ Brachytherapy inserts radioactive seeds directly in or around a tumour to deliver dose.

${ }^{8} \mathrm{I}$ was not directly involved in the assembly of the prototype, though I provided some assistance in its evaluation. I do not consider that work part of the main body of my research.
} 


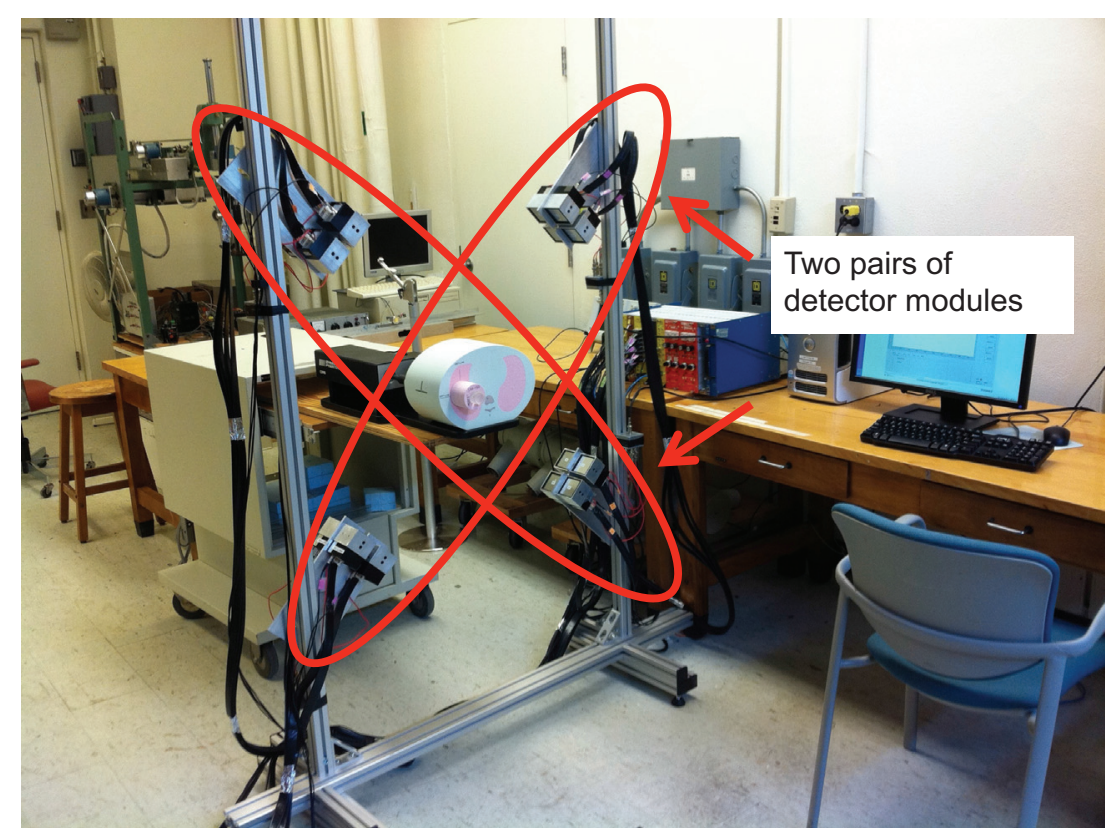

Figure 2.7: Prototype PeTrack system mounted on aluminum frame.

(PMT; Hamamatsu H8500, Hamamatsu Photonics K.K., Iwata City, Shizuoka Pref., Japan). The crystal blocks and PMTs are housed within a light-tight aluminum enclosure. A 1-mm thick aluminum window covers the front face of the crystal arrays. A single crystal element measures $3.9 \times 3.9 \times 20 \mathrm{~mm}^{3}$. The distance between the centre of two consecutive crystal elements is $4 \mathrm{~mm}$. Each detector module pair is read out by an MPET-8 position calculation and coincidence unit (mesytec GmbH \& Co KG, Putzbrunn, Germany). The two MPET-8 readout units are connected to a single mesytec MCPD-2 central processing device connected by Ethernet to a personal computer.

\subsubsection{Energy resolution}

Performance evaluation of a preliminary version of the prototype detectors was conducted by Soleimani. [3] The energy resolution was measured as (22 \pm 1$) \%$ at $511 \mathrm{keV}$ from a set of ten crystal elements from a detector block. Figure 2.8 (reproduced from [3] with permission from the author) shows the energy spectrum measured by 


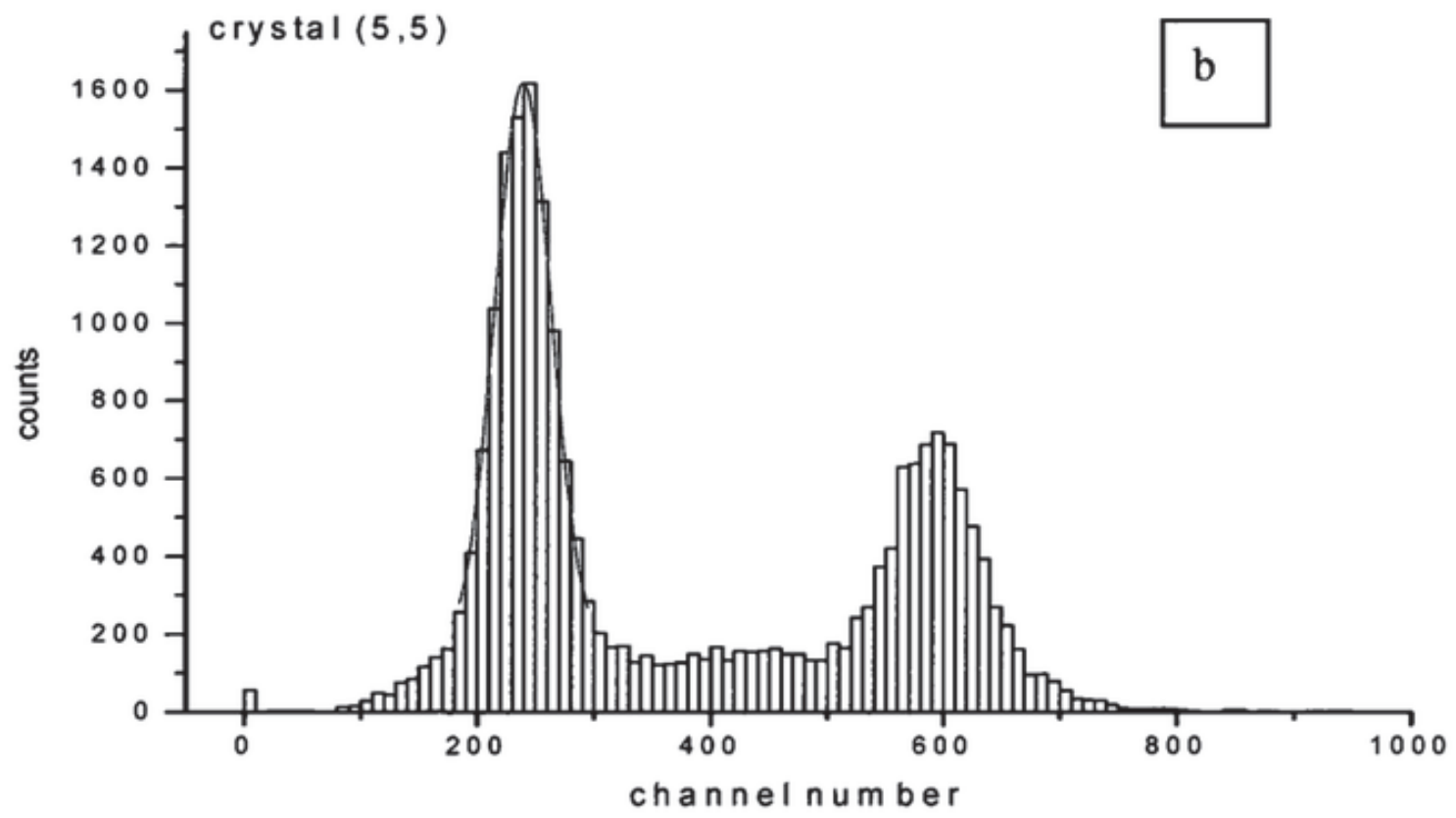

Figure 2.8: Energy spectrum of a single crystal element (size $3.9 \times 3.9 \times 20 \mathrm{~mm}^{3}$ ) exposed to radiation from a ${ }^{22} \mathrm{Na}$ source. The peak around channel 230 is the $511 \mathrm{keV}$ annihilation peak; the peak around channel 600 is the $1.274 \mathrm{MeV}$ photopeak of ${ }^{22} \mathrm{Na}$. This figure is reproduced from [3] with permission from the author.

a single crystal element $\left(\right.$ size $\left.3.9 \times 3.9 \times 20 \mathrm{~mm}^{3}\right)$ exposed to radiation from a ${ }^{22} \mathrm{Na}$ source. The peak around channel 230 is the $511 \mathrm{keV}$ annihilation peak; the peak around channel 600 is the $1.274 \mathrm{MeV}$ photopeak of ${ }^{22} \mathrm{Na}$. Similarly, Figure 2.9 shows the energy spectrum measured by one of the detector modules (i.e. the sum of the energy spectrum of each crystal element in the detector module) when exposed to radiation from a ${ }^{22} \mathrm{Na}$ source placed near the isocentre of the system. The energy resolution is approximately $27 \%$ at $511 \mathrm{keV}$ (near channel 375). The difference in energy resolution with the single crystal element and the set of 10 crystal elements is due to widely non-uniform gain across PMTs. ${ }^{9}$

\footnotetext{
${ }^{9}$ Unpublished internal report.
} 


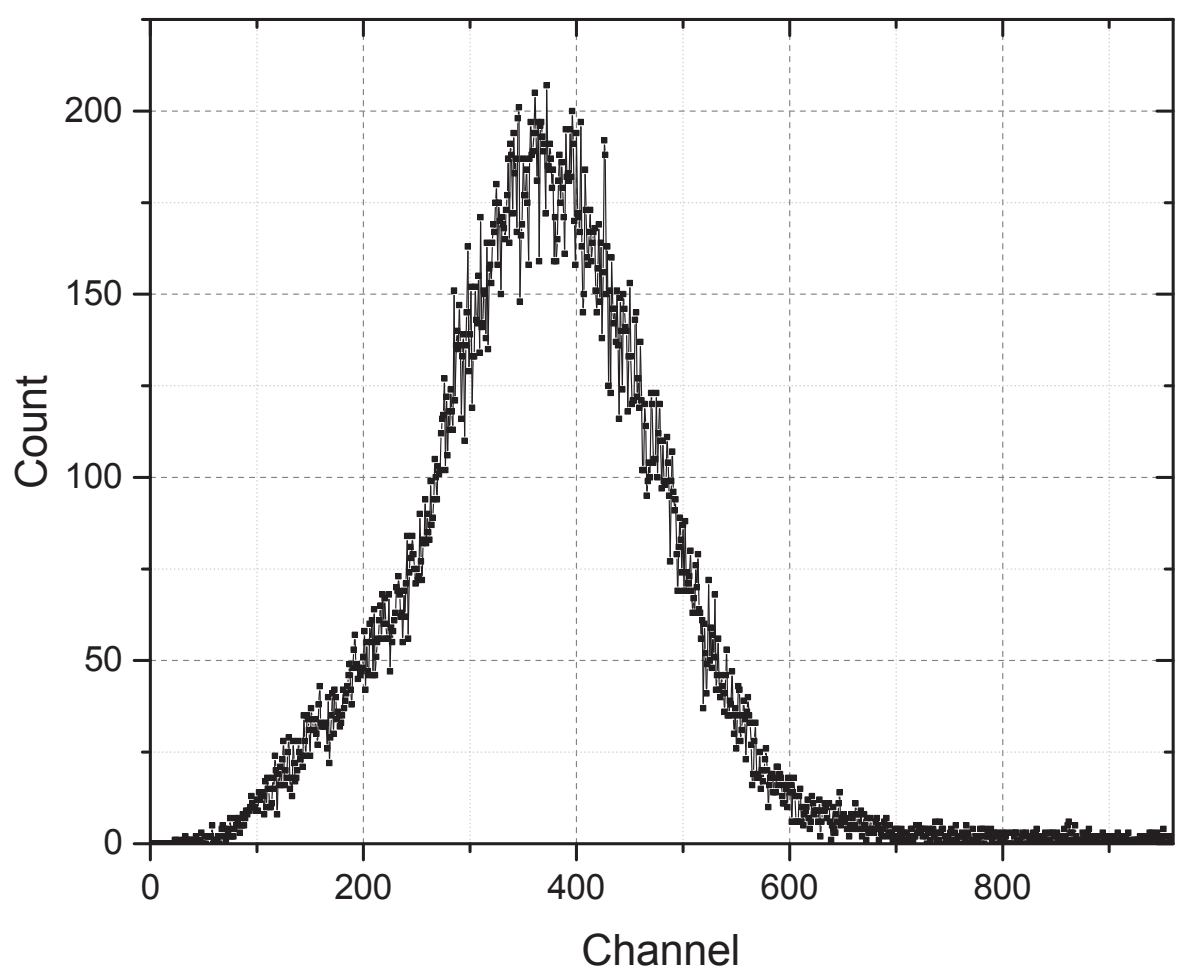

Figure 2.9: Energy spectrum of a detector module exposed to radiation from a ${ }^{22} \mathrm{Na}$ source. The peak near channel 375 is the $511 \mathrm{keV}$ annihilation peak. 


\subsubsection{Crystal identification map}

In order to correctly identify the location of recorded events, the response from the detector needs to be segmented into the individual crystal elements that comprise the detector block. To that end, a ${ }^{22} \mathrm{Na}$ source was placed close to the surface of each detector block and a 2D histogram of the position of the recorded events (called a flood histogram) was generated for each detector block. Figure 2.10 shows the flood histogram for a sample detector block. Each crystal element can clearly be identified on this map, with the exception of the four corner crystals possibly due to lower sensitivity of the PMT in those regions. In addition, two crystals in the top row appear fused together. This may be due to a lack of reflective coating between the two crystals. Figure 2.11 shows $1 \mathrm{D}$ profiles of a row and of a column of crystal elements from the flood histogram. All 12 crystals are clearly distinguishable along each direction, with a good peak-to-valley ratio. Non-uniform gain of the PMT causes the crystals in the centre to have a lower intensity than crystals closer to the edges. More details about this calibration procedure can be found in [3].

\subsubsection{Sensitivity and accuracy of the prototype system}

The sensitivity of the prototype PeTrack detector system was studied by Razavi. [32] The sensitivity is approximately 120 counts per second (cps) per microcurie of activity at the isocentre of the system. At $3.5 \mathrm{~cm}$ from isocentre in the axial direction, the sensitivity is approximately $60 \mathrm{cps} / \mu \mathrm{Ci}$. Detailed maps of the sensitivity can be found in [32]. The effective FOV of the system is approximately $11 \times 11 \mathrm{~cm}^{2}$. The average localization error within the FOV is $0.5 \mathrm{~mm}$. The accuracy of the localization by PeTrack depends, among other factors, on the alignment of the detector modules. Misalignment of the modules introduces a systematic error in the direction of the misalignment. If the misalignment is known accurately, the error can be accounted 


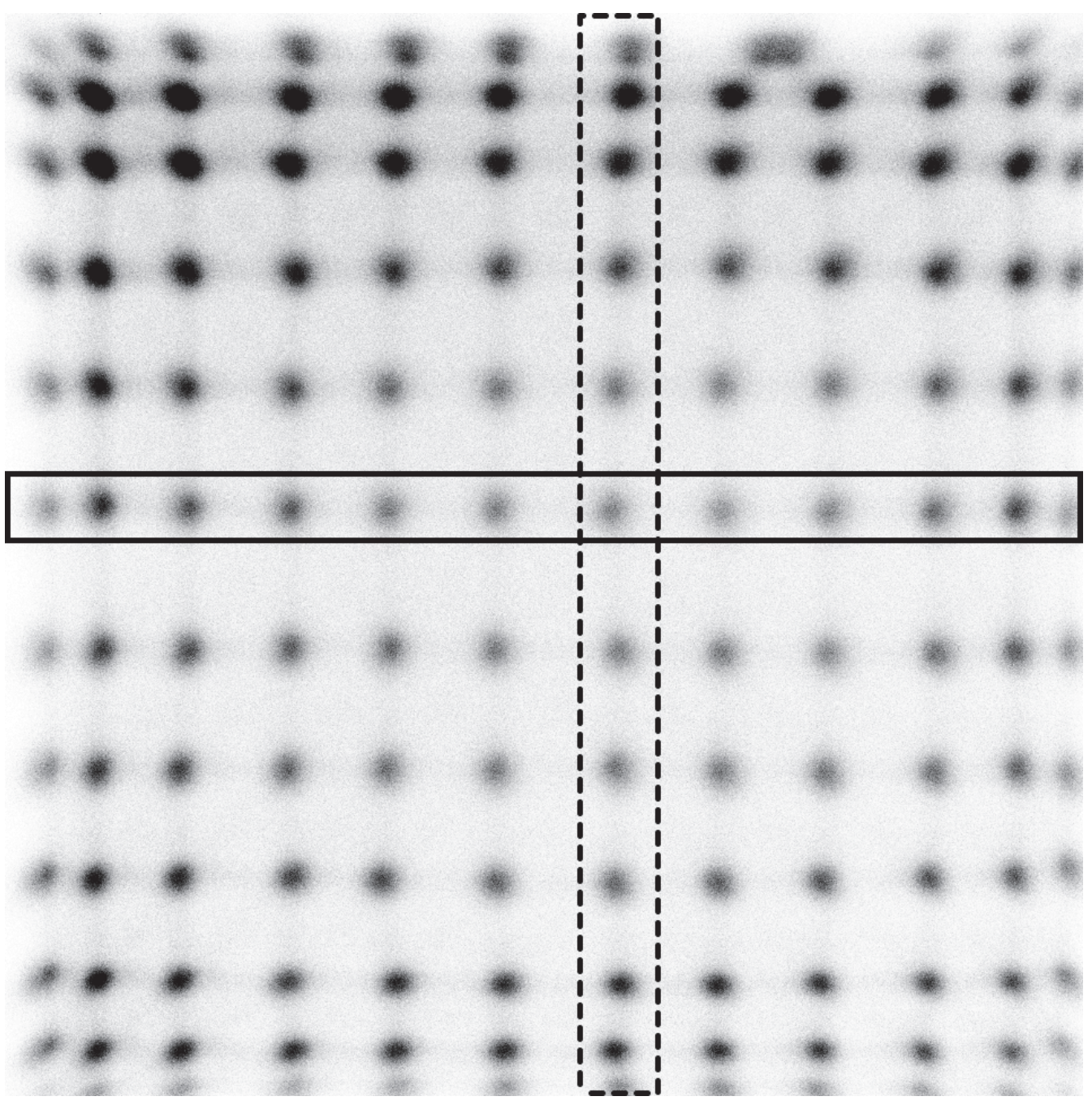

Figure 2.10: Sample 2D flood histogram from a prototype PeTrack detector block (see Section 2.2.8) exposed to radiation from a ${ }^{22} \mathrm{Na}$ source placed close to the surface of the detector. Each crystal element is clearly distinguishable with the exception of the four corner crystals, possibly due to lower sensitivity of the PMT in those regions. In addition, two crystals in the top row appear fused together. This may be due to a lack of reflective coating between the two crystals. Profiles of this figure along the horizontal (solid rectangle) and vertical (dashed rectangle) directions were measured. 


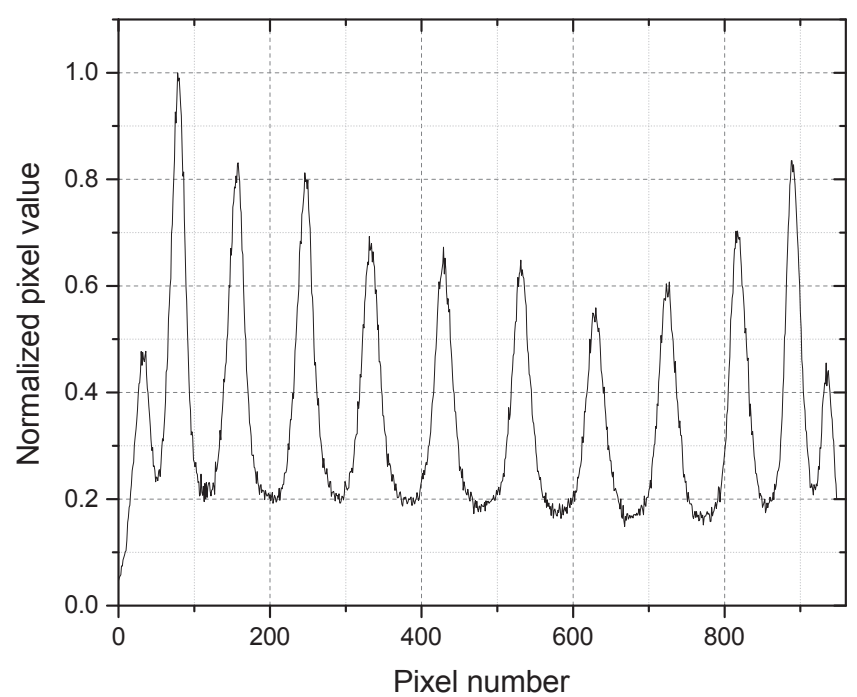

(a) Horizontal profile

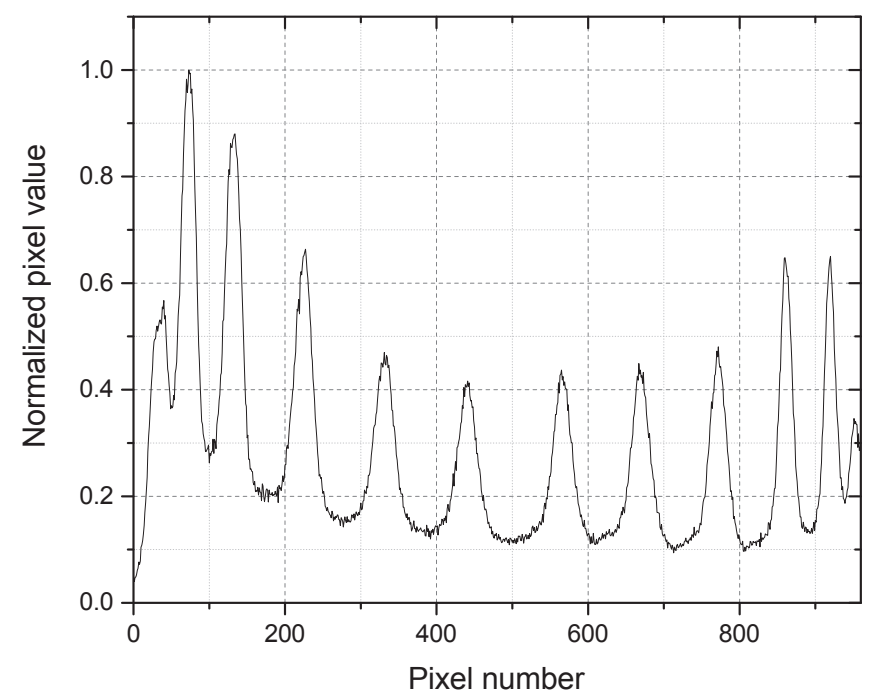

(b) Vertical profile

Figure 2.11: One dimensional profile of a row (a) and a column (b) of crystal elements, as measured on the 2D flood histogram (see Figure 2.10. All 12 crystals are clearly distinguishable along each direction, with a good peak-tovalley ratio. Non-uniform gain of the PMT causes the crystals in the centre to have a lower intensity than crystals closer to the edges. 
for by the system (for example, as a correction applied between localizations of the marker). The sensitivity study also revealed a scaling distortion across the FOV which causes a $1 \%$ contraction of lengths along the $x, y$, and $z$ axes of the coordinate system.

\subsection{Conclusion}

This chapter presented an overview of PeTrack, a real time tracking system using fiducial positron emission markers. The tracking algorithm and prototype detector system of PeTrack were described and quantified in terms of their performance. The next chapter will provide a proof of concept application of respiratory-gated radiation therapy using the prototype PeTrack system. 


\section{Chapter 3}

\section{Respiratory gating in radiation therapy using PeTrack}

\subsection{Introduction}

Section 1.1 motivated the need for motion tracking in radiation therapy. The most common use of motion tracking is to apply respiratory gating. In respiratory gated radiotherapy, the beam delivery is synchronized with the displacement or phase of the recorded motion. The goal is to deliver the beam only when the target volume is within a pre-determined window.

Spirometry was used early to provide a breathing signal in radiotherapy. [39, 40] A spirometer measures the volume and air flow rate from the lungs. However, spirometers require patients to breathe only from the mouth for extended periods of time, which may prove uncomfortable for some patients.

Another system used is the Anzai AZ-773V Respiratory Gating System (Anzai Medical, Tokyo, Japan). [41,42] It consists in a pressure sensitive belt worn around the patient's abdomen or thorax. Sensors in the belt record the pressure changes due to expansion of the abdomen or thorax during breathing. This provides an external surrogate signal for gating. 
The most commonly used motion sensing system for gating is by far the Varian Real-time Position Management ${ }^{\mathrm{TM}}$ (RPM) system (Varian Medical Systems, Palo Alto, CA). [41-46] The RPM system comprises an infrared sensitive camera surrounded by infrared-emitting LED lights. A block with two passive reflective markers facing the camera is placed on the patient's abdomen. The patient's breathing causes the marker block to move primarily in the AP direction. The camera detects the motion of the marker block by capturing the reflected light. The sampling rate of the system is $30 \mathrm{~Hz}$. More recently, the system was upgraded to include six markers on the block, which allows the RPM to track external surrogate 3D motion.

It is important that an external surrogate breathing signal be well correlated with internal target volume motion. Given the variability of breathing patterns between patients and even between and within treatment sessions, the correlation between external and internal motion may vary widely in quality. [44,47-51]

To avoid bad correlations between the external and internal signals, tumour motion may be tracked directly. Gold fiducial markers implanted in or around a target volume can be tracked in real time by fluoroscopy. [52] The location of the small spherical markers is automatically determined on x-ray images acquired at a rate up to $30 \mathrm{~Hz}$. However, the estimated skin dose delivered by a fluoroscopic tracking system is between 0.3 and 1.2 Gy per hour of treatment. [53] As a reference, a typical radiotherapy treatment duration is 10 minutes per weekday for four weeks at The Ottawa Hospital, but treatment length can vary with tumour size and several other factors. Efforts need to be made to manage and limit such radiation doses. Fluoroscopic tracking of smaller cylindrical markers with a length of $3 \mathrm{~mm}$ and a diameter of $0.8 \mathrm{~mm}$ has been reported with a maximum tracking error varying between 0.2 and $2.3 \mathrm{~mm}$ at a $95 \%$ confidence level. [54] 
The ExacTrac 6DRT system (BrainLAB AG, Feldkirchen, Germany) combines external motion detection by optical markers with internal position detection by kilovoltage $\mathrm{x}$-ray imaging of the target volume. $[45,55]$ The $\mathrm{x}$-ray images are fused to the setup computed tomography (CT) images. During treatment, the infrared external markers are used for external marker motion tracking and gating, whereas the x-ray images are used to confirm positioning of the patient periodically. The ExacTrac system also supports tracking of internal fiducial markers, but doing so would increase the radiation dose to the normal tissues of the patient since x-ray images would need to be acquired at a higher frequency.

Similarly, the CyberKnife Robotic Radiosurgery System (Accuray, Inc., Sunnyvale, CA) system uses its own Synchrony Respiratory Motion Tracking System (RMTS) to estimate an internal-external motion correlation model. $[37,56] \mathrm{Cy}-$ berKnife also uses a stereocamera to track the motion of three external optical markers attached to the abdomen or chest of the patient. The 3D location of implanted gold fiducial markers is determined from two kilovoltage x-ray images acquired every 1 to 5 minutes. Between the periodic x-ray imaging, the system relies on the correlation between the external marker and internal motion to infer the real-time location of the tumour. However, the correlation is not alway stable. The main advantage of the CyberKnife over other methods is that it can dynamically reposition the treatment beam to follow the motion of the target volume.

The Mitsubishi Vero4DRT system (MHI-TM2000 Linear Accelerator, Mitsubishi Heavy Industries, Ltd., Tokyo, Japan) can also dynamically follow the tumour motion in real time. [57-59] The Vero4DRT uses two orthogonal kilovoltage x-ray imaging systems and an infrared camera to track implanted and external markers, in a manner similar to the CyberKnife. The motion of the tumour can be followed in real time with a gimballed linac head. The compact $6 \mathrm{MV}$ linac head is mounted inside a circular gantry and can rotate around the treatment couch. The gimballed head can 
rotate in the tilt and pan directions which allows the beam to to swing by $\pm 41.9 \mathrm{~mm}$ in either direction at isocentre.

Other systems rely only on internal motion tracking. The Calypso System (Varian Medical Systems, Palo Alto, CA) uses an external magnetic source and receiver coil array to track the location of implanted wireless coils in the patient. [60] In a patient study, the 3D difference between the location of the transponders determined by the Calypso system and the radiographic localization of the markers was $1.5 \pm 0.9 \mathrm{~mm}$. [61] The main drawback of this technique is the large size of the transponders $(8.7 \mathrm{~mm} \times$ $1.85 \mathrm{~mm}$ ), which increases patient risk during implantation. In addition, transponders are not reusable. They may also cause artefacts on magnetic resonance imaging (MRI) or CT images. [62] The distance between the receiver array and the emitter coils is recommended to be smaller than $27 \mathrm{~cm}$, which may be hard to achieve for thicker patients.

The Micropos 4DRT system (Micropos Medical, Göteborg, Germany) also uses electromagnetic transponders for real time position monitoring. [63] Patient studies suggest an accuracy of $2.7 \pm 1.2 \mathrm{~mm}$. However, the transponders are wired to the system, which complicates implantation and may cause patient discomfort.

The RealEye system (Navotek Medical Ltd., Yokneam, Israel) uses an implanted radioactive fiducial marker for real time patient positioning and monitoring during radiotherapy. [64] The tracking is based on the detection of gamma rays from a single implanted gamma emitter. Three rotating collimated detectors are placed around the patient. Each detector has a planar field of view (FOV) which allows it to point in the direction of the source. The intersection of the planes of the three detectors define the location of the marker. The system is limited to tracking a single marker at a time.

Positron emission guided radiation therapy (EGRT) was recently proposed for tumour tracking. [65,66] The EGRT system consists of a transverse full ring circular 
gantry which contains two arcs of opposing PET detectors, a compact linear accelerator (linac) head, and megavoltage x-ray detectors. Prior to treatment, the patient is injected with FDG, which is taken up by the tumour. Ignoring scatter and random coincidences, LORs recorded by the PET detectors pass through the target volume. Thus, when an LOR is recorded, the system rapidly rotates around its isocentre and points the linac head along the LOR. A very small beam of radiation is then delivered along the LOR. In practice, a unique EGRT algorithm (which takes into account scatter, random coincidences, attenuation, and many other effects) determines if recorded LORs qualify for radiation delivery. The performance and accuracy of the system depend on the target-to-background activity uptake ratio.

\subsubsection{PeTrack for tumour tracking in radiotherapy}

PeTrack has been proposed for real time 3D tumour tracking in radiotherapy. [25] The tracking information can be used for respiratory gated radiotherapy. The PeTrack detector system will be mounted on the gantry of a linac (see Figure 3.1). One or more fiducial markers described in Section 2.2.7 can be implanted by biopsy needle in or around a target volume. The small size of the markers facilitate implantation and minimize risk to the patient. The radioisotope and the activity of the marker should be selected based on the expected treatment duration, thickness of the patient, and desired tracking frequency (i.e. how many times per second should the markers be located). For example, Xu et al. [25] have shown that if the markers are to be located every $100 \mathrm{~ms}$ with a success rate of 99.5\%, 62 coincidence events per marker need to be used if the estimated initial location of the markers is off by $10 \mathrm{~mm}$. This number of coincidence events can be recorded within $100 \mathrm{~ms}$ by using markers of activity 3.7 MBq each, assuming the coincidence photons travel through $30 \mathrm{~cm}$ of tissue. The radiation dose from the markers to the patient can also be taken into account during treatment planning. [25] 


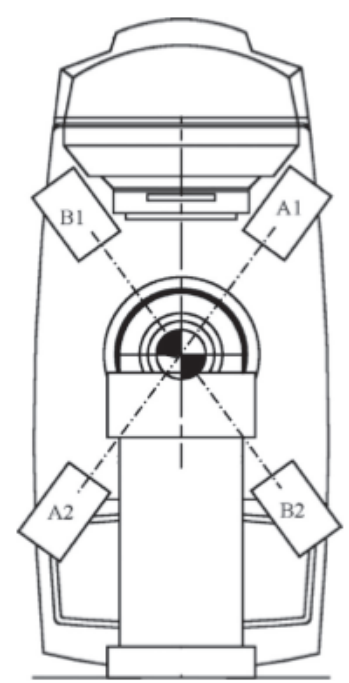

Figure 3.1: PeTrack detector modules (A1, A2, B1, and B2) mounted on a linac gantry. Reproduced with permission from AAPM.

This chapter will present a proof of concept application of PeTrack for respiratory gated radiotherapy. First, the scattered radiation to which the PeTrack detectors will be exposed during radiotherapy was quantified by means of Monte Carlo simulations. Second, the prototype PeTrack system was integrated with a commercially available linac used for research purposes as an experimental respiratory-gated radiation therapy system. Radiochromic film measurements were made to evaluate the feasibility of respiratory gating using PeTrack.

\subsection{Radiation environment of PeTrack in radiation therapy}

The application of PeTrack to respiratory gating in radiation therapy requires reliable gamma ray detectors that can work in the radiation environment of medical linacs. These linacs produce high energy x-ray beams (4 MV to $25 \mathrm{MV}$ ) to deliver radiation dose to tumours. However radiation scattered from the patient and treatment bed 
will reach the nearby PeTrack detectors. In this section, the characteristics of the scattered radiation reaching the detectors are quantified and the dose deposited in the BGO crystals is calculated.

\subsubsection{EGSnrc simulation}

The EGSnrc code system is a Monte Carlo simulation package for the transport of electrons and photons through any element, compound, or mixture. [67] It has been extensively validated and is widely considered the gold standard in terms of accuracy in the medical physics field. EGSnrc is designed for the transport of particles of energies in the range of $1 \mathrm{keV}$ to a few hundred $\mathrm{GeV}$. Several physical processes are implemented in EGSnrc, such as Bremsstrahlung production, positron annihilation, multiple scattering of charged particles, scattering of photons, pair production, etc. A user code tailored to the radiation transport problem at hand was written with the EGSnrc C ++ class library (EGS ++ for short). [68]

\section{Geometry definition}

The geometry of the situation was defined using the geometry module of EGS ++ . A $30 \times 30 \times 30 \mathrm{~cm}^{3}$ water phantom was placed on a $50 \times 50 \times 10 \mathrm{~cm}^{3}$ graphite table. The prototype PeTrack detector blocks were modelled as BGO blocks, surrounded by a Teflon (PTFE) light shield, encased in an aluminum frame. Figures 3.2 and 3.3 show the details of the geometry and the final placement of the four detector modules.

\section{Scoring options}

Absorbed dose to a medium, $D$, is defined as:

$$
D=\frac{d \bar{\epsilon}}{d m}
$$




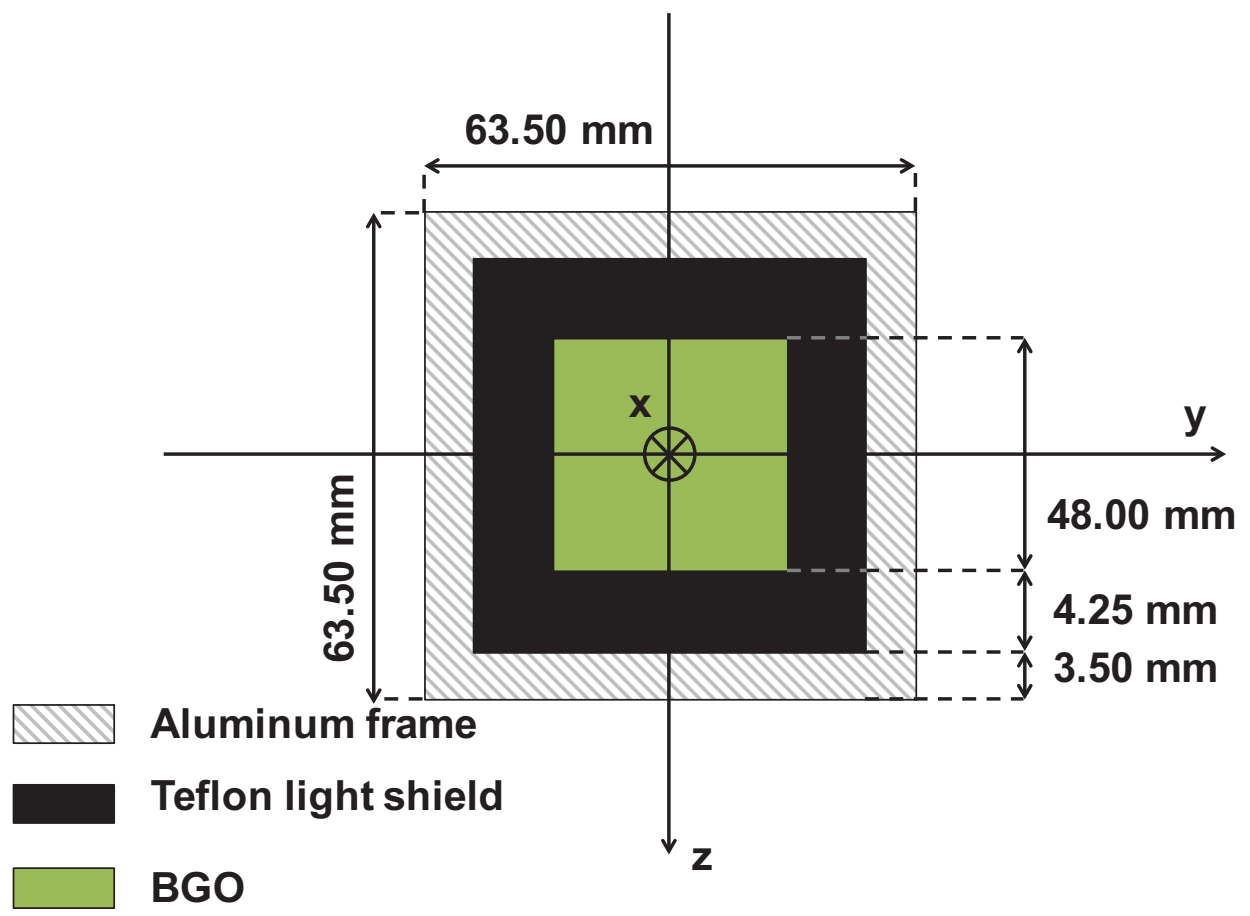

(a) Front view

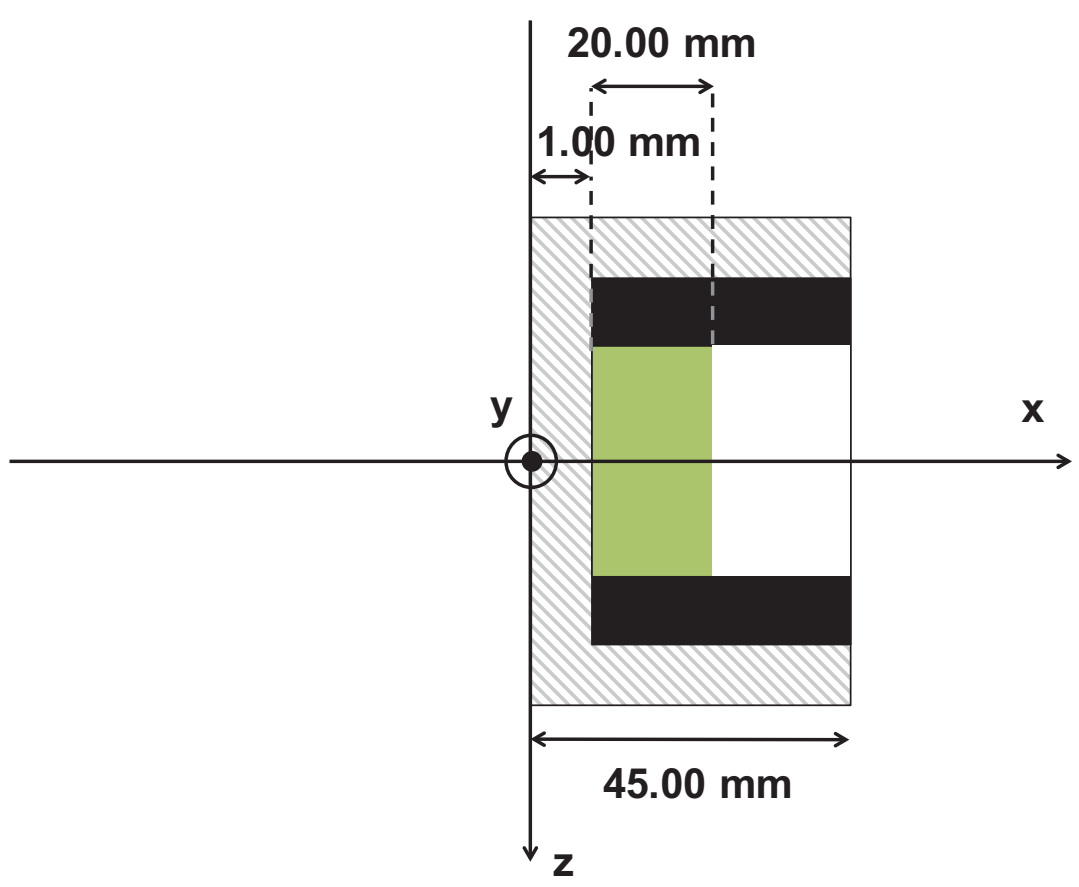

(b) Side view

Figure 3.2: Design of the detector blocks. Figures are not to scale. 


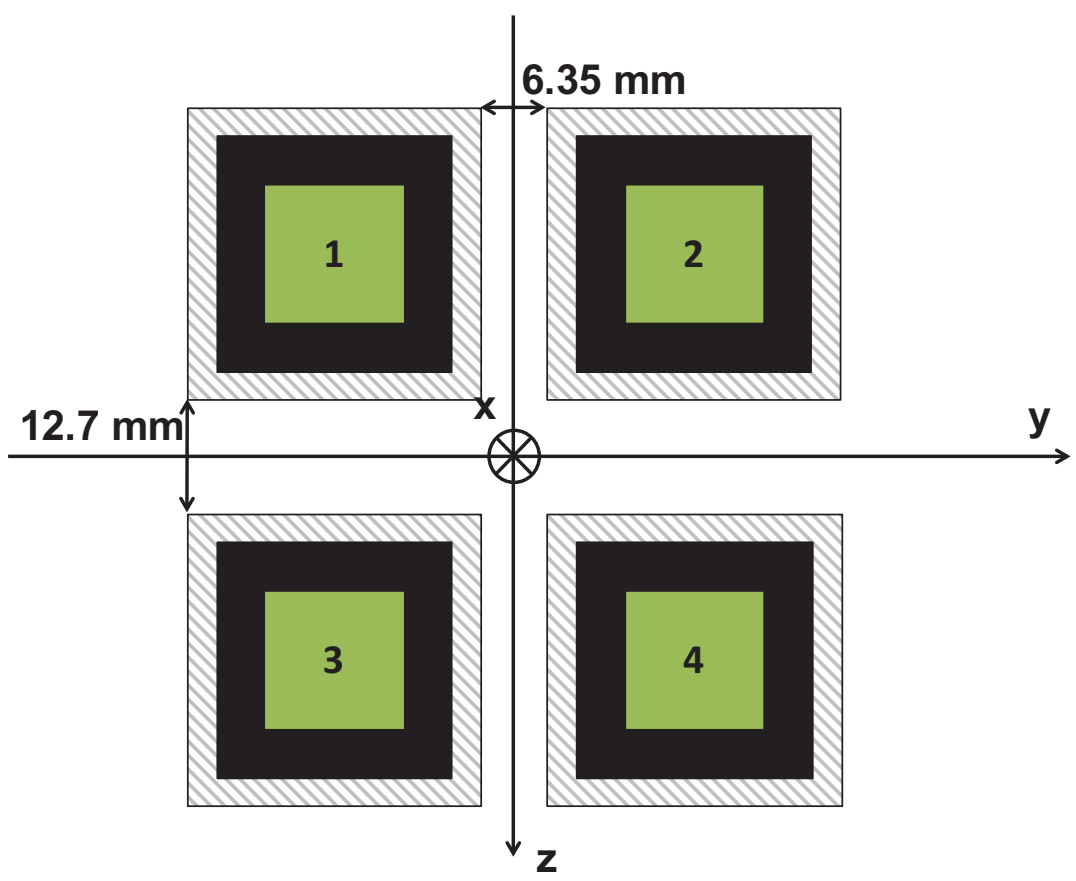

(a) Design of the modules

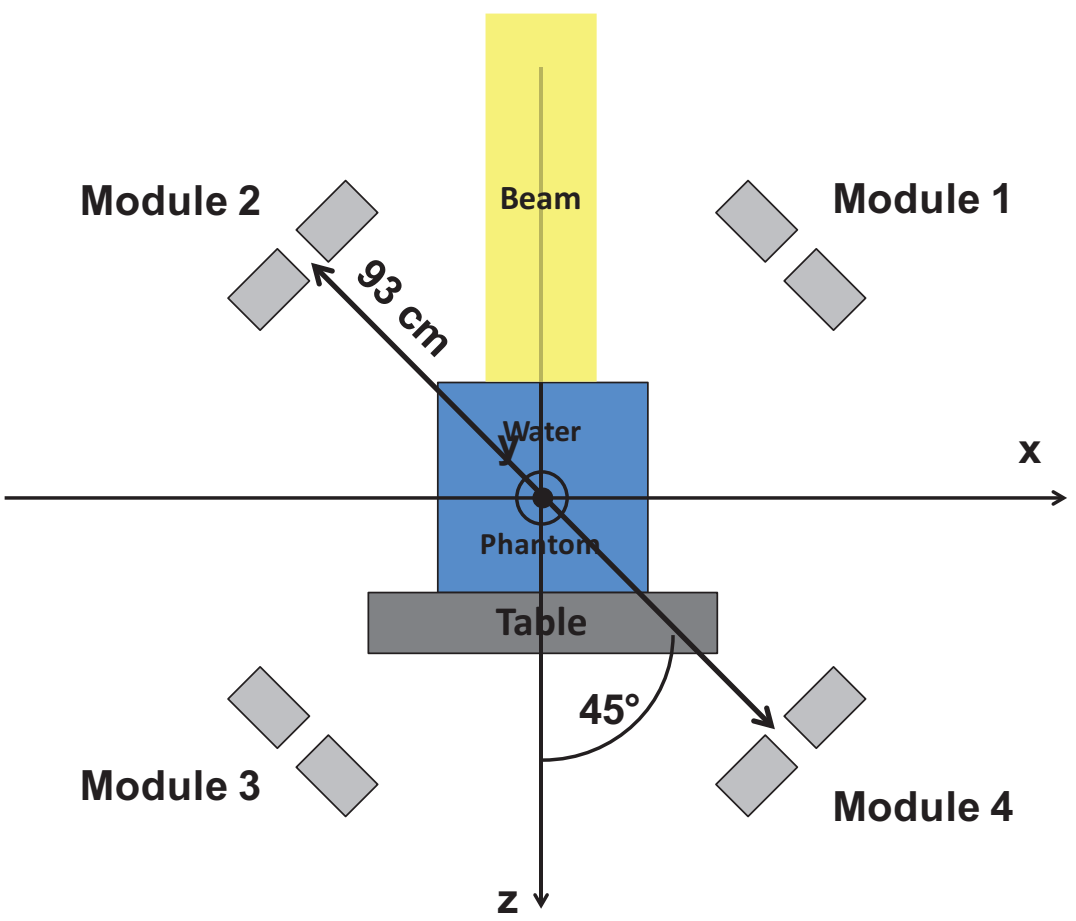

(b) Final geometry placement

Figure 3.3: Design of the modules and final geometry placement. Figures are not to scale. 
where $d \bar{\epsilon}$ is the mean energy deposited to a medium of infinitesimal mass $d m$. Absorbed dose is measured in Grays (Gy), with $1 \mathrm{~Gy}=1 \mathrm{~J} / \mathrm{kg}$. Absorbed dose is a quantity only defined at a point. If we are interested in the dose to a volume, the average dose, $\bar{D}$, is used instead:

$$
\bar{D}=\frac{\epsilon_{t o t}}{M}
$$

where $\epsilon_{t o t}$ is the total energy imparted to a volume of mass $M$. The average dose due to scatter to the BGO crystals in the PeTrack detectors is expected to be small. Since there is symmetry of the geometry around the central axis of the beam, the average dose to BGO in the upper (\#1 and \#2) and lower (\#3 and \#4) modules and the dose along the central axis in the water phantom (in $1 \mathrm{~cm}^{3}$ voxels) were scored. The maximum dose along the central axis of the water phantom, $D_{\max }$, was used as a reference. The fluence differential in energy of the scattered photons hitting the aluminum window of the detector modules was also scored. By definition, the fluence at point $P$ is:

$$
\Phi=\frac{d N}{d a}
$$

where $d N$ is the number of particles incident on an infinitesimal sphere of crosssectional area $d a$ surrounding point $P$. This fluence is independent of the angle of incidence of the radiation. ${ }^{1}$ There is an equivalent definition of fluence which is more straightforward to implement in Monte Carlo simulations:

$$
\Phi=\frac{\Sigma_{\text {tracks }} l}{V}
$$

where $\Phi$ is the fluence at point $P$ and $l$ is the track length of particles through a volume $V$ centered at point $P$. This second definition was used to calculate the fluence of scattered photons at the centre of the aluminum window of the PeTrack

\footnotetext{
${ }^{1}$ On the other hand, planar fluence is the number of particles crossing a plane per unit area. Planar fluence depends on the angle of incidence of the radiation.
} 
detectors. The fluence to both the upper and lower modules was scored. All scored quantities were normalized to the total fluence of the source. In EGS++, the fluence of a parallel beam source is defined as the total number of simulated histories divided by the area of the shape of the beam.

\section{Source definition}

For the calculation of the dose to the BGO, the phantom was irradiated with a parallel beam of photons of field sizes $5 \times 5,10 \times 10,15 \times 15$, and $20 \times 20 \mathrm{~cm}^{2}$. The energy spectrum of photons produced in a 10-MV accelerating potential Varian Clinac linac and measured by Mohan et al. was used. [69]. This photon spectrum is provided with the EGSnrc distribution. For a given field size of $10 \times 10 \mathrm{~cm}^{2}$, the effect of beam energy was evaluated using the 4, 6, 10, 15, and 24 MV spectra by Mohan et al. Calculations of the energy spectrum and fluence of the scattered photons used a $10 \times 10 \mathrm{~cm}^{2}$ field size and accelerating potentials 6,10 , and $24 \mathrm{MV}$.

\section{Monte Carlo transport parameters}

The transport of electrons was terminated if the electron's total energy (including rest mass energy) dropped below $700 \mathrm{keV}$. Terminating the transport of an electron immediately deposits all remaining energy of the electron locally, which saves computing time. The CSDA range of electrons in water of total energy $700 \mathrm{keV}$ or less is smaller than $0.05 \mathrm{~mm}$. Thus, terminating their transport at that energy has little to no impact on the physical validity of the simulation. Similarly, photon transport was terminated if photon energy dropped below $10 \mathrm{keV}$. The PRESTA-I boundary crossing algorithm for electron transport was used because the geometry had very few boundaries and high accuracy was not critical. All other parameters used their default value. 


\section{Variance reduction}

The efficiency $\epsilon$ of Monte Carlo simulations in radiotherapy is customarily defined as $[70-73]$ :

$$
\epsilon=\frac{1}{T_{C P U} s^{2}}
$$

where $s^{2}$ is an estimate of the true variance on a quantity of interest and $T_{C P U}$ is the total CPU time required to obtain this variance. Hence a relatively small estimated variance $s^{2}$ or short computing time $T_{C P U}$ are characteristic of an efficient (large $\epsilon$ ) Monte Carlo simulation. A variance reduction technique (VRT) increases the efficiency (i.e. reduces the variance or the computing time) of a Monte Carlo simulation while producing the same results as without a VRT. Since only scattered photons were of interest, two VRT were used: radiative splitting with a splitting number of $N_{\text {split }}=175$ and Russian roulette $(\mathrm{RR})$. Radiative splitting causes a bremsstrahlung event to produce $N_{\text {split }}$ photons each with statistical weight $1 / N_{\text {split }}$, increasing the number of secondary photons reaching the scoring regions (i.e. the detectors). When electrons are produced from photon interactions, RR randomly terminates their transport. Electrons have a survival probability of $1 / N_{\text {split }}$ in this process. The statistical weight of electrons which survive RR is increased by a factor $N_{\text {split }}$. This prevents wasting computing time tracking electrons which are not directly relevant to the situation under study.

\section{Run control}

For dose calculations, simulations were run until either $1 \%$ dose accuracy was reached in the third voxel along the central axis of the phantom ${ }^{2}$ or 50 million histories had been simulated. For energy spectrum and fluence calculations, simulations were run until 10 billion histories had been simulated.

\footnotetext{
${ }^{2}$ This voxel is close to the maximum dose depth.
} 


\subsubsection{Results}

The average dose to the BGO in the lower modules, $D_{\text {lower }}$, was expressed as a percentage of $D_{\max }$. With a $10 \times 10 \mathrm{~cm}^{2}$ field size and accelerating potentials 4 , 6, 10, 15, and $24 \mathrm{MV}$ respectively, $D_{\text {lower }}$ varied between 0.10 and $0.06 \%$ of $D_{\max }$ (Figure 3.4, bottom left). The average dose to the BGO in the upper modules, $D_{\text {upper }}$, was expressed as a fraction of $D_{\text {lower }}$ (Figure 3.4 , upper left). With field sizes of $5 \times 5,10 \times 10,15 \times 15$, and $20 \times 20 \mathrm{~cm}^{2}, D_{\text {lower }}$ varied between 0.02 and $0.37 \%$ of $D_{\max }$ (Figure 3.4 , bottom right). Finally, $D_{\text {upper }}$ as a fraction of $D_{\text {lower }}$ is shown in Figure 3.4 (upper right). The BGO in the lower modules receives a larger average dose than the BGO in the upper modules. This is expected as the lower modules are downstream from the phantom. According to the differential electronic cross section for the Compton effect ${ }^{3}$, higher energy photons tend to scatter in the forward direction, whereas lower energy photons have comparatively more chance to scatter at an angle greater than $90^{\circ}$ relative to their incident direction. Thus, the lower modules are exposed to more higher energy photons than the upper modules. The dose is also shown to decrease with nominal beam energy because scatter is more forward directed at higher energies.

In all cases studied, the average dose to the BGO crystals was very small compared to $D_{\max }$. As an estimate of the dose to the BGO after a day of treating patients in the clinic, assume a clinic has a linac calibrated to deliver 1 Gy at dose maximum for a $10 \times 10 \mathrm{~cm}^{2}$ field at $100 \mathrm{~cm}$ SSD (source-surface distance) for $100 \mathrm{MU}$. The clinic treats 30 patients per day with $600 \mathrm{MU}$ per fraction ${ }^{4}$ and a $10 \mathrm{MV} 10 \times 10 \mathrm{~cm}^{2}$ photon beam. The average dose to the lower modules would be approximately $30\left[\frac{\text { fractions }}{\text { day }}\right] \times$

\footnotetext{
${ }^{3}$ See, for example, E.B. Podgoršak, Radiation Physics for Medical Physicists, Springer, Berlin, 2010, pp. 309-312. [26]

${ }^{4}$ The number of monitor units per fraction varies widely between treatment method, site, machine output, etc. Compare with MU per fraction values reported in [74-76].
} 

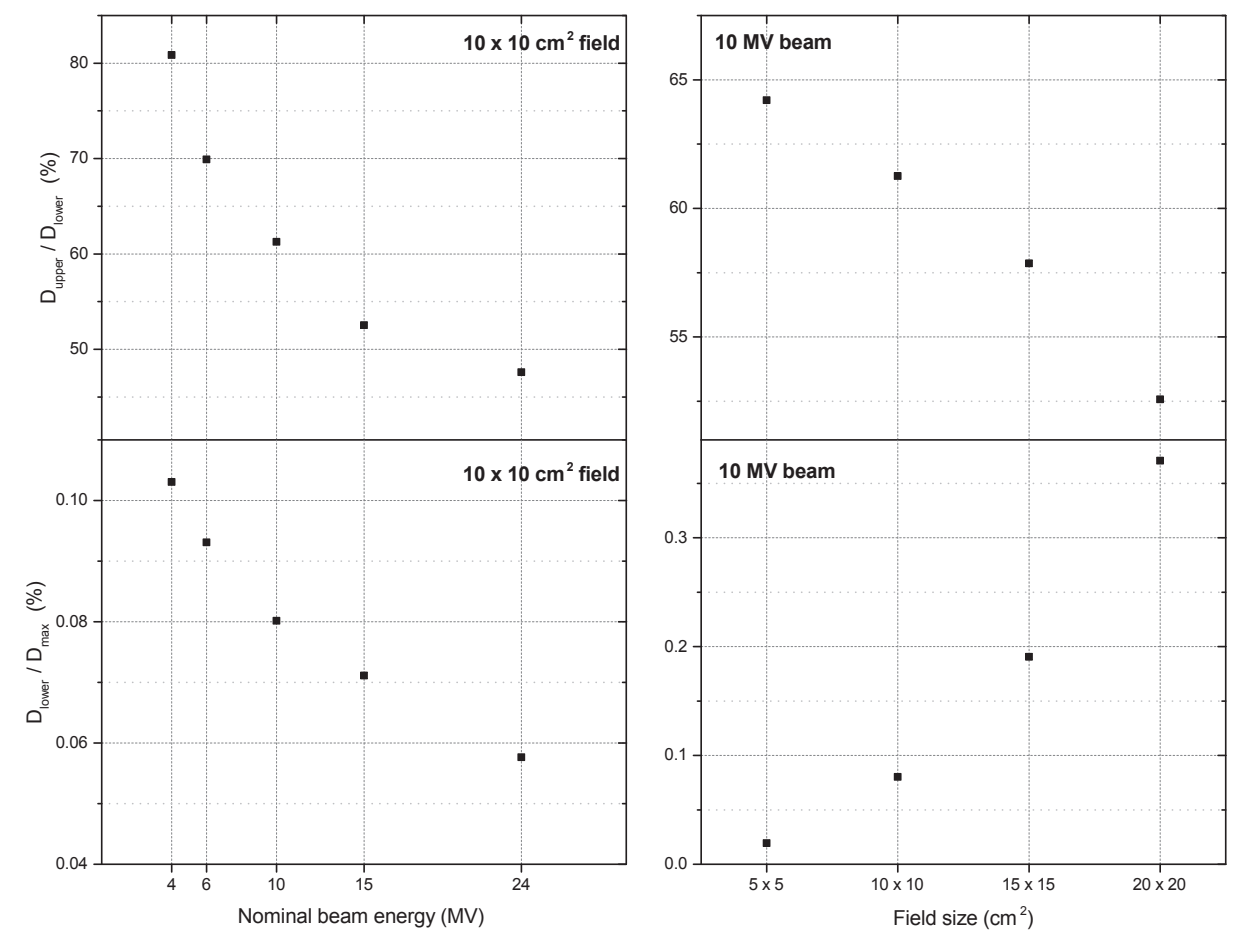

Figure 3.4: Average dose to the upper modules relative to the lower modules (top left) and average dose to the lower modules relative to the maximum dose along the central axis of the phantom (bottom left) for various nominal beam energies and field size $10 \times 10 \mathrm{~cm}^{2}$. The same quantities for nominal beam energy $10 \mathrm{MV}$ and various field sizes are shown on the right. 
$600\left[\frac{\mathrm{MU}}{\text { fraction }}\right] \times 1\left[\frac{\mathrm{Gy}}{100 \mathrm{MU}}\right] \times 0.0008=1.44 \mathrm{cGy}$ per day, where 0.008 is the average dose to the lower modules relative to the $D_{\max }$.

The fluence of scattered photons at the centre of the aluminum window is plotted in Figures 3.5 and 3.6. The fluence was binned into 2-keV bins between 0 and $1 \mathrm{MeV}$ and into $250 \mathrm{keV}$ bins between 1 and $10 \mathrm{MeV}$. The fluence was normalized to the total fluence of the source. For the upper modules, $95 \%$ of the fluence consists of photons with energy below $350 \mathrm{keV}$; similarly, 95\% of the scattered photons reaching the lower modules have energy below $1.5 \mathrm{MeV}$.

Two peaks occur at $77 \mathrm{keV}$ and $87 \mathrm{keV}$ in the fluence distribution: they correspond respectively to the $\mathrm{K} \alpha_{2}$ and $\mathrm{K} \beta_{1}$ transitions of bismuth. The peak at $511 \mathrm{keV}$ is an annihilation photon peak. When the energy of a photon exceeds $2 m_{e} c^{2}=1.022 \mathrm{MeV}$, photons can interact by pair production: an electron-positron pair is created and the photon is absorbed in the medium. The electron and positron annihilate and produce two $511 \mathrm{keV}$ annihilation photons. 


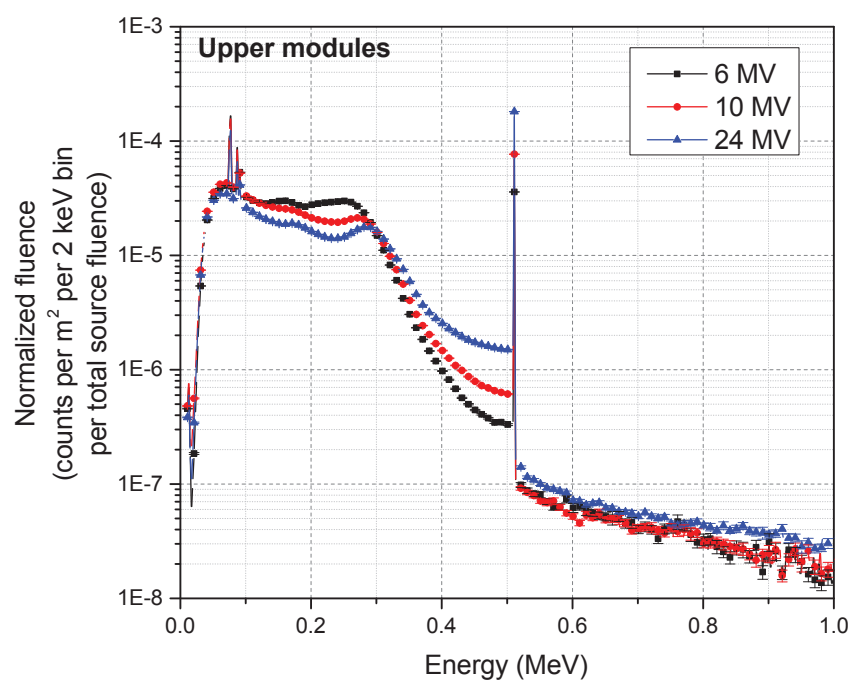

(a) Upper modules

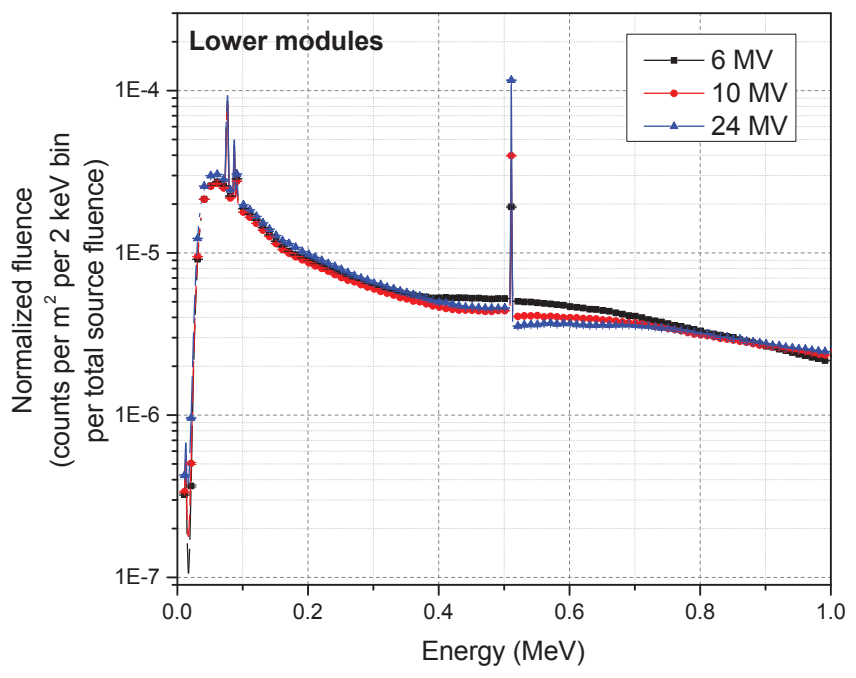

(b) Lower modules

Figure 3.5: Fluence at the centre of the aluminum window of the upper modules (a) and lower modules (b) for energies up to $1 \mathrm{MeV}$, in $2 \mathrm{keV}$ wide bins. The fluence was normalized to the total fluence of the source. In EGS++, the fluence of a parallel beam source is defined as the total number of simulated histories divided by the area of the shape of the beam. The peaks at $77 \mathrm{keV}$ and $87 \mathrm{keV}$ correspond to the characteristic $\mathrm{x}$ rays of bismuth. The peak at $511 \mathrm{keV}$ is an annihilation peak. For the sake of visual clarity, five data points are skipped between each point on the graph. 


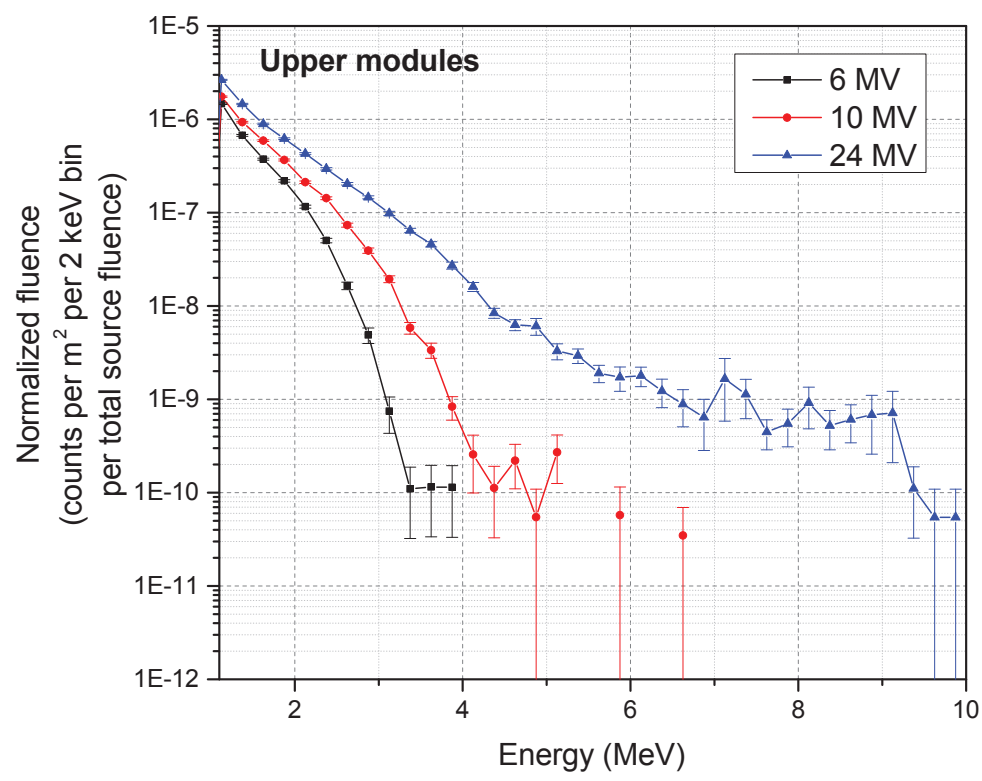

(a) Upper modules

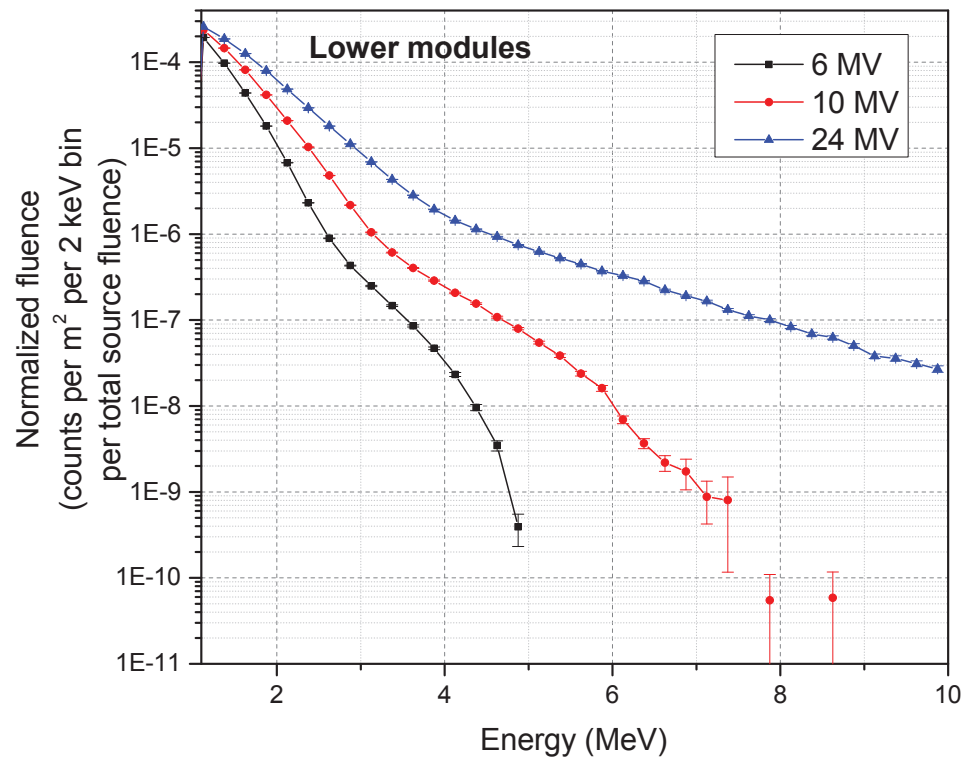

(b) Lower modules

Figure 3.6: Fluence at the centre of the aluminum window of the upper modules (a) and lower modules (b) for energies between 1 and $10 \mathrm{MeV}$, in $250 \mathrm{keV}$ bins. The fluence was normalized to the fluence of the source. In EGS++, the fluence of a parallel beam source is defined as the total number of simulated histories divided by the area of the shape of the beam. 


\subsection{Experimental evaluation of respiratory gating with PeTrack}

A proof of concept of respiratory gating using PeTrack was conducted at the National Research Council (NRC) of Canada. The PeTrack system was used in conjunction with the NRC Elekta Precise linac. The prototype detector system described in Section 2.2 was mounted on an aluminum frame under the linac (see Figure 3.7). An anthropomorphic dynamic thorax phantom was used to provide realistic patient breathing patterns (see Section 3.3.3). A single fiducial marker was used to track motion along the central axis of the phantom. Delivery of the beam was interrupted when the target moved out of a predetermined gating window. The delivered dose distribution was measured on radiochromic films. Figure 3.8 illustrates the setup. The two MPET-8 modules acquire coincident events from the two pairs of detector modules. The MCPD-2 collects the events and sends them to a PC. Each detector module is equipped with a high voltage gating device which turns the PMT HV off during every linac x-ray pulse (see Section 3.3.2). The gating of the HV is based on a pulsing signal from the linac which is regulated by a pulse generator. An electrometer (Model 6517A, Keithley Instruments, Inc., Cleveland, OH) reads the signal from an ion chamber placed within the beam at the exit from the phantom and sends it to the PC, through an analog-to-digital converter (ADC). 


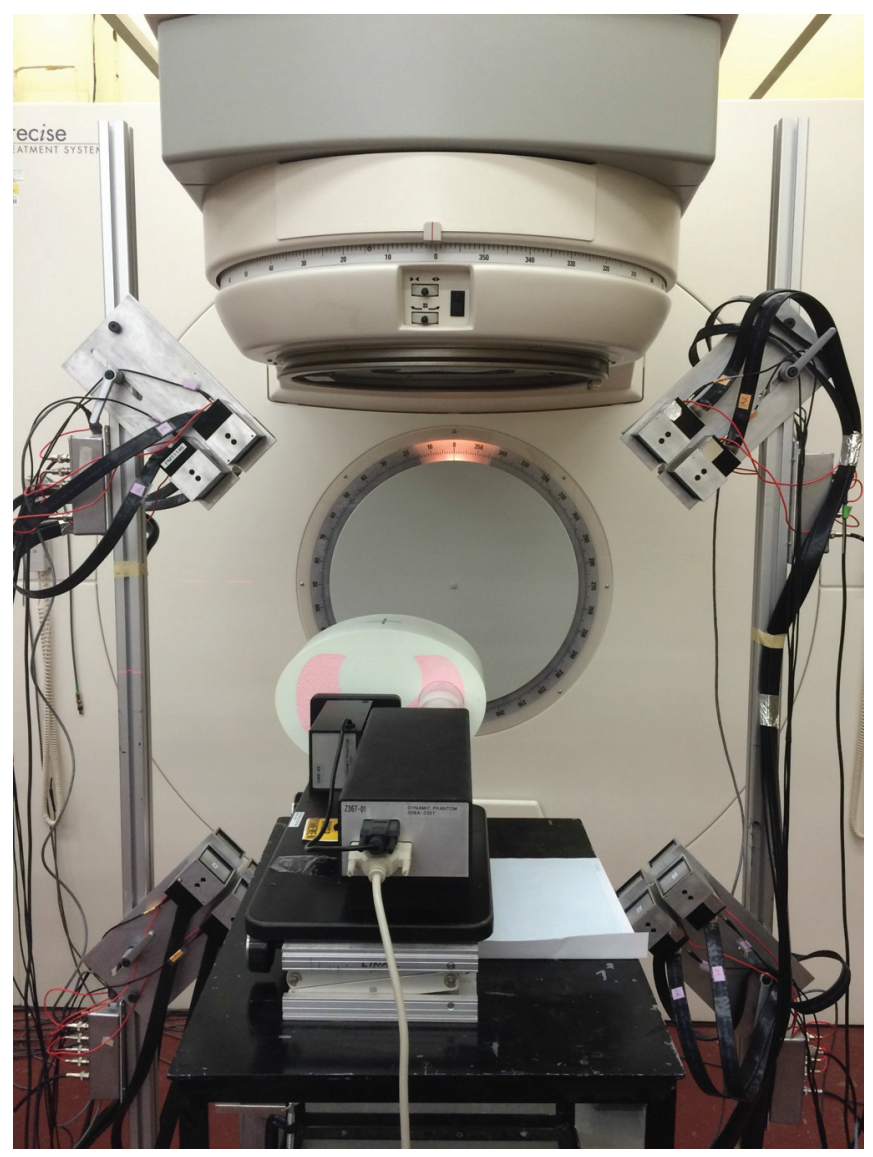

Figure 3.7: The prototype PeTrack detectors mounted on an aluminum frame under the head of a linac. An anthropomorphic dynamic thorax phantom sits on the treatment table. 


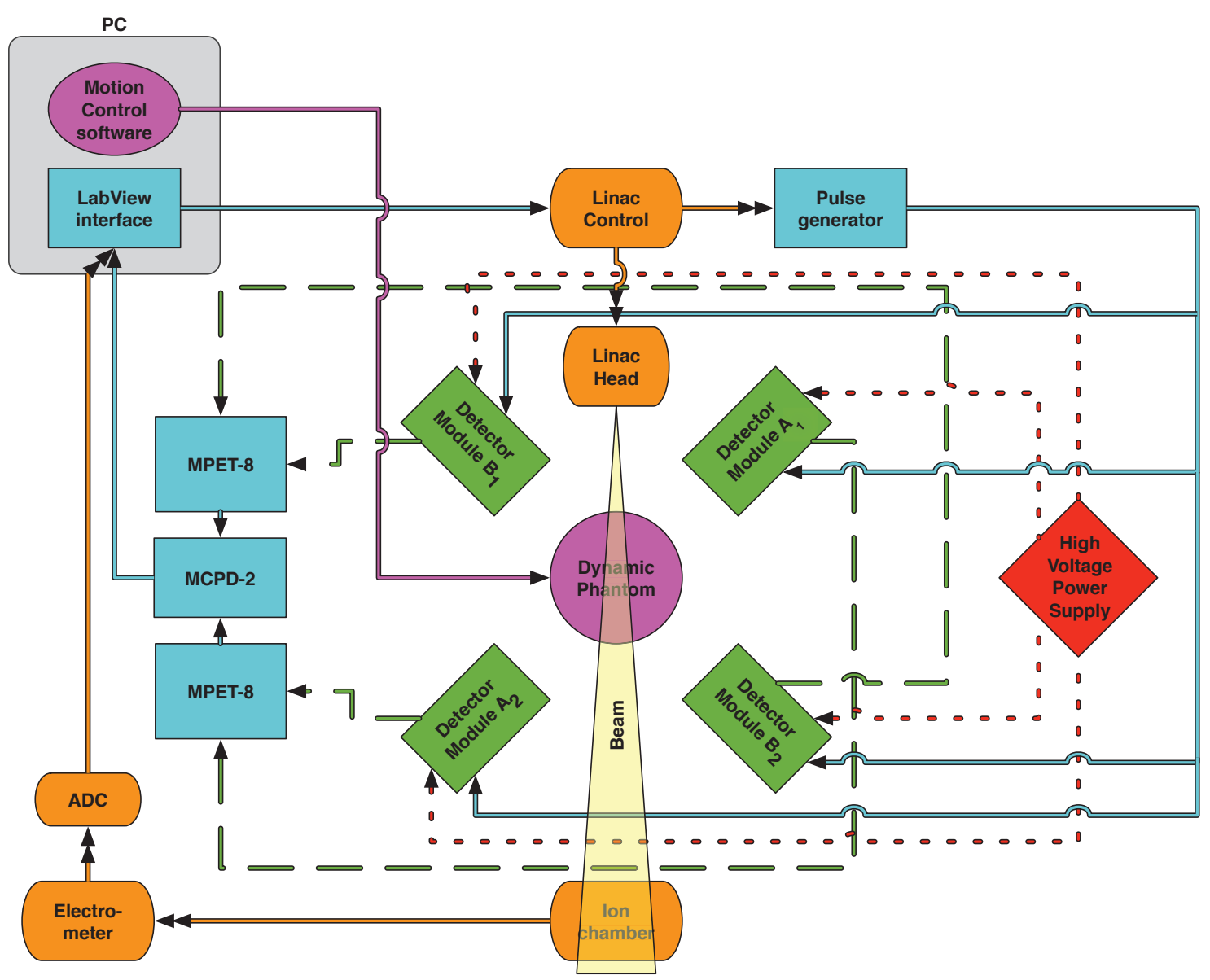

Figure 3.8: Overview of the experimental setup. The two MPET- 8 modules acquire coincident events from the two pairs of detector modules. The MCPD-2 collects the events and sends them to a PC. Each detector module is equipped with a high voltage gating device which turns the PMT HV off during every linac x-ray pulse based on a pulsing signal from the linac, regulated by a pulse generator. An electrometer reads out the signal from the ion chamber. The reading from the electrometer is sent out to a $\mathrm{PC}$, through a digital-to-analog converter. 


\subsubsection{PeTrack linac gating software}

Software to control the gating of the linac was developed in LabVIEW (National Instruments Corporation, Austin, TX). ${ }^{5}$ The software was designed to serve several roles:

- it remotely controls the detector system (e.g. adjustable gain, data acquisition, etc.), processes coincidence events, and delivers them to the tracking component of the interface;

- it tracks the 3D location of the markers in real time using the PeTrack algorithm;

- it provides a graphical user interface (GUI) to adjust the tracking parameters (number of markers being tracked, sampling frequency, etc.) and a live 3D display of the location of the markers (see Fig. 3.9);

- it provides a gating interface which can hold the beam of a linear accelerator and provides a GUI for the gating parameters (gating window, status of the beam, live display of the location of the markers, etc.) (see Fig. 3.10).

The gating interface runs on a personal computer (PC) with an Intel Core 2 Quad processor. The gating interface was specifically designed to communicate with the NRC Elekta Precise linac. When PeTrack determines that a marker has moved outside the predetermined gating window, a command is sent via the serial port from the gating interface to the linac to interrupt the pulse repetition frequency (PRF) chain, effectively holding the beam. Once the marker moves back into the gating window, a command is sent to the linac to turn the beam back on. Issues

\footnotetext{
${ }^{5}$ Dr. Tong $\mathrm{Xu}$ assisted me in the development of the LabVIEW interface. Dr. Xu handled the components of the interface which establishes communication with the MPET-8 modules. I wrote the LabVIEW VIs which deliver and process the raw events from the detector system to the computer. I adapted the VIs from the open source program mesydaq. I designed and wrote all the LabVIEW VIs directly related to the gating of the Elekta linac. I also ported the PeTrack tracking code into a dynamically linked library (DLL) compatible with LabVIEW.
} 


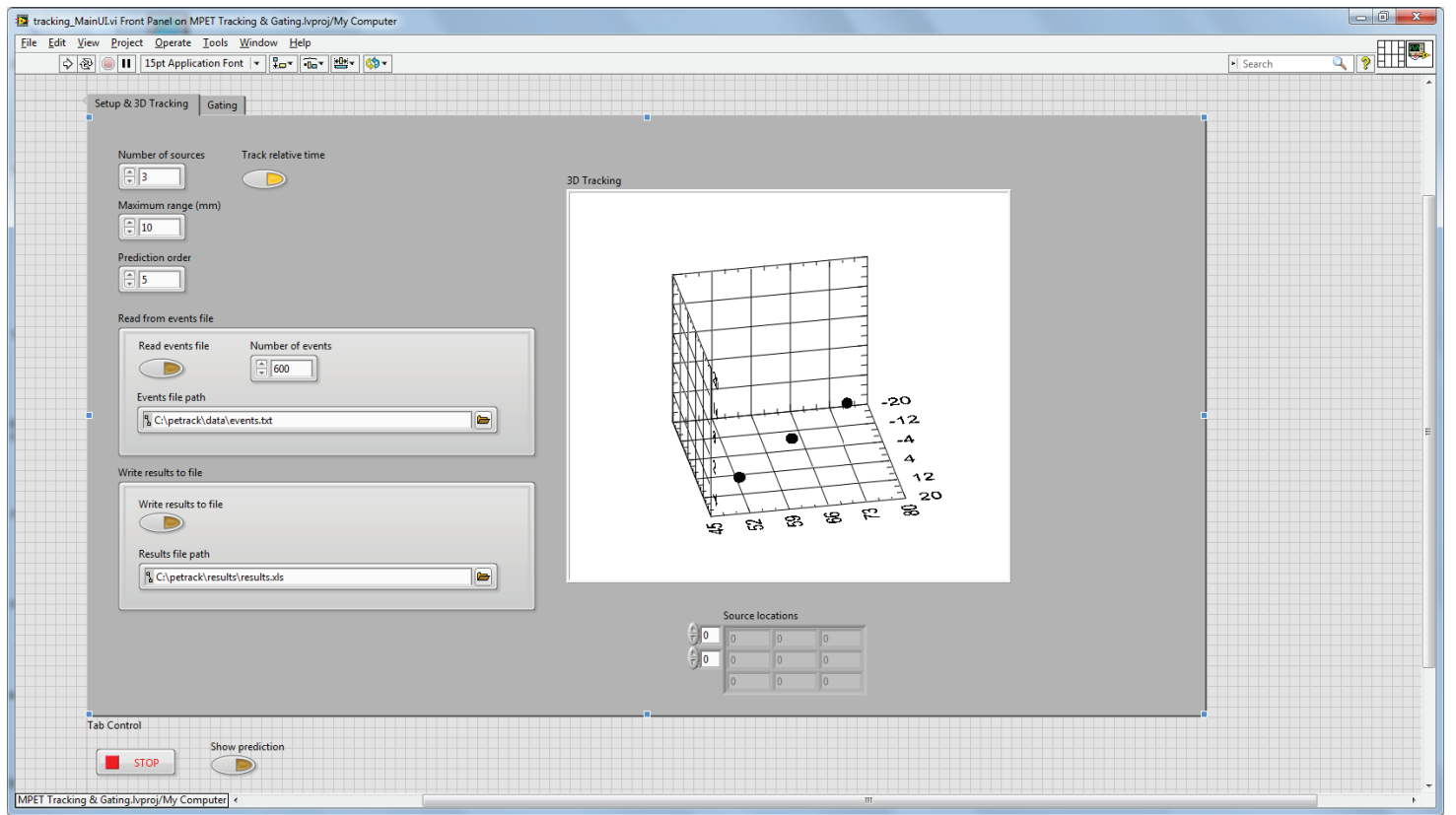

Figure 3.9: The GUI of the tracking component allows users to control various parameters such as the sampling frequency and the prediction order.

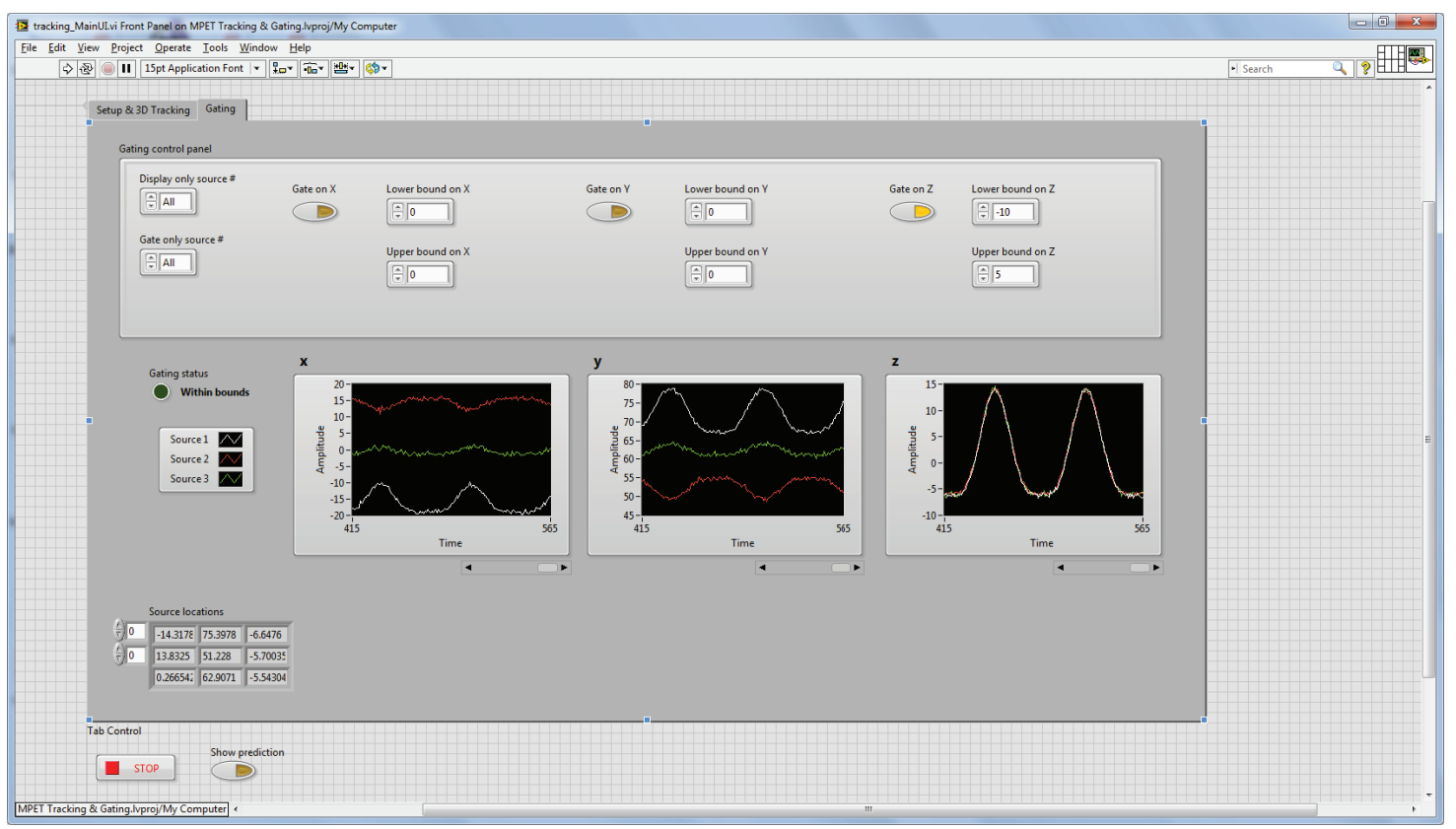

Figure 3.10: The GUI of the gating component allows users to set the size of the gating window and monitor the status of the beam delivery. 
with the inherent latency of the tracking and beam delivery system are addressed in Section 3.3.4.

\subsubsection{Gating of the PeTrack detectors}

During delivery of radiotherapy treatments, linacs operate in pulsed mode. The pulse rate falls in the range 50 to $400 \mathrm{~Hz}$ and the pulse width is a few microseconds $(<10 \mu \mathrm{s})$. The signal from a positron emission marker is much lower than the intense scattered $\mathrm{x}$ rays from a linac pulse. In addition, the scattered $\mathrm{x}$ rays generate intense scintillation light in the PeTrack detectors which saturates the PMTs. [3] Figure 3.11 (reproduced from [3] with permission from the author) shows the output signal of a sample PMT (coupled to a single $2 \times 2 \times 2 \mathrm{~cm}^{3}$ BGO crystal) due to scattered $\mathrm{x}$ rays from a $6 \mathrm{MV}$ photon beam incident on a $30 \times 30 \times 22 \mathrm{~cm}^{3}$ water phantom for various field sizes. As the field size increases, more scattered photons will reach the detectors and the PMT output should increase with the field size. However Figure 3.11 shows that the PMT output varies very little with the field size. This indicates that the PMT is saturated by the scintillation light from the scattered radiation. Therefore, the PeTrack detectors need to be gated off during each linac x ray pulse. A custom phase-lock loop (PLL) system was designed and built through a contract with TECFA Inc. (Ottawa, ON). The device is based on a field-programmable gate array (FPGA). It monitors the signal from the pulse forming network (PFN) of the linac, automatically synchronizes with it, and generates an approximately $90-\mu$ s wide gating window roughly centred around the linac pulse. Within that gating window, the negative high voltage that powers the PMTs is turned off. The linac PFN starts generating pulses before any $\mathrm{x}$ rays are produced; this allows the PLL to detect the frequency of the linac x-ray pulses and synchronize with it before the beam turns on. Figure 3.12 shows sample oscilloscope traces of the gating of the PeTrack detectors under operation. 


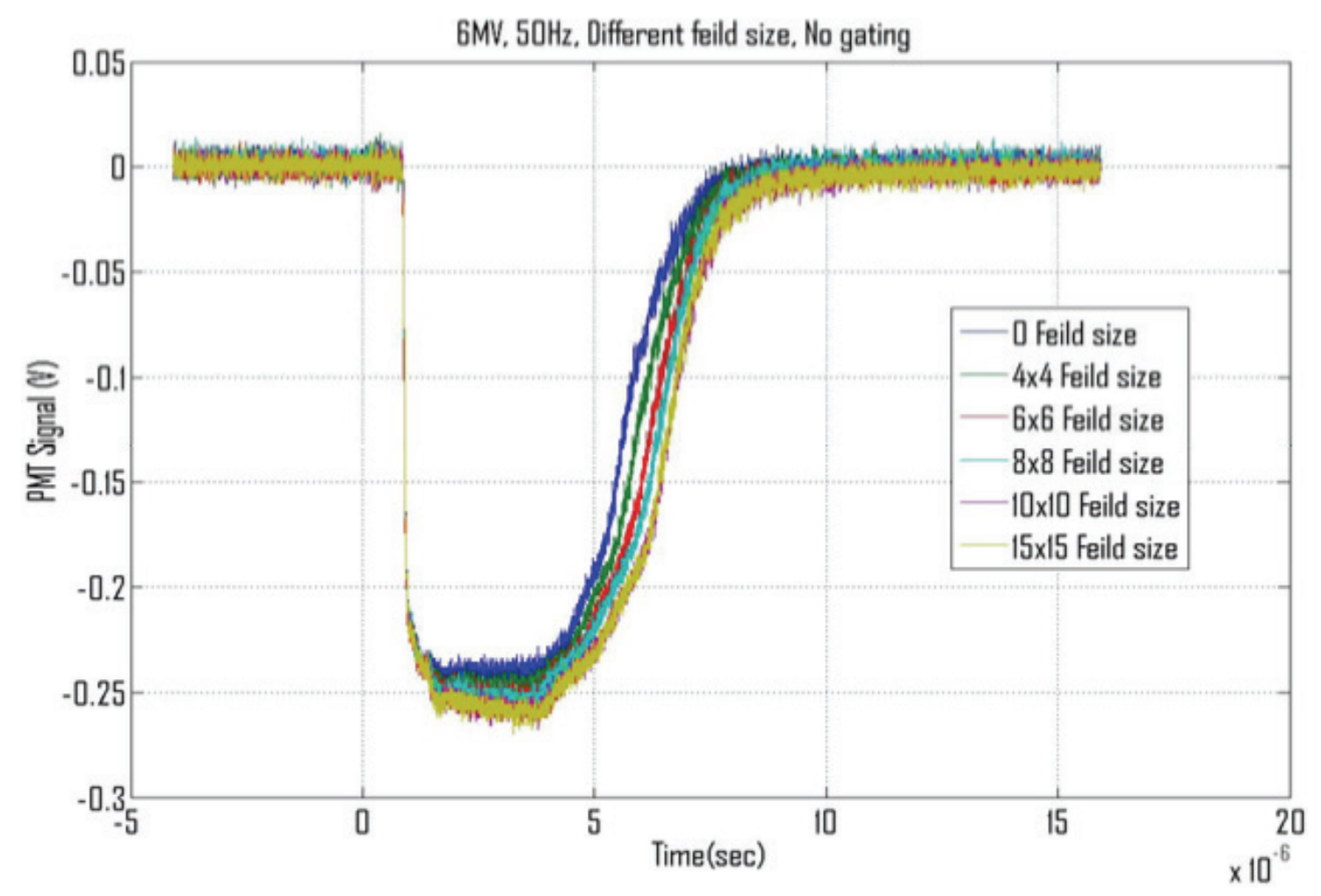

Figure 3.11: PMT output due to scattered $\mathrm{x}$ rays from a $6 \mathrm{MV}$ photon beam incident on a $30 \times 30 \times 22 \mathrm{~cm}^{3}$ water phantom for various field sizes. As the field size increases, more scattered $x$ rays reach the scintillator crystal coupled to the PMT. The signal from the PMT should increase with the field size. However, the figure shows that the PMT output varies very little with the field size. This indicates that the PMT is saturated by the scintillation light from the scattered radiation. This figure was reproduced from [3] with permission from the author. 


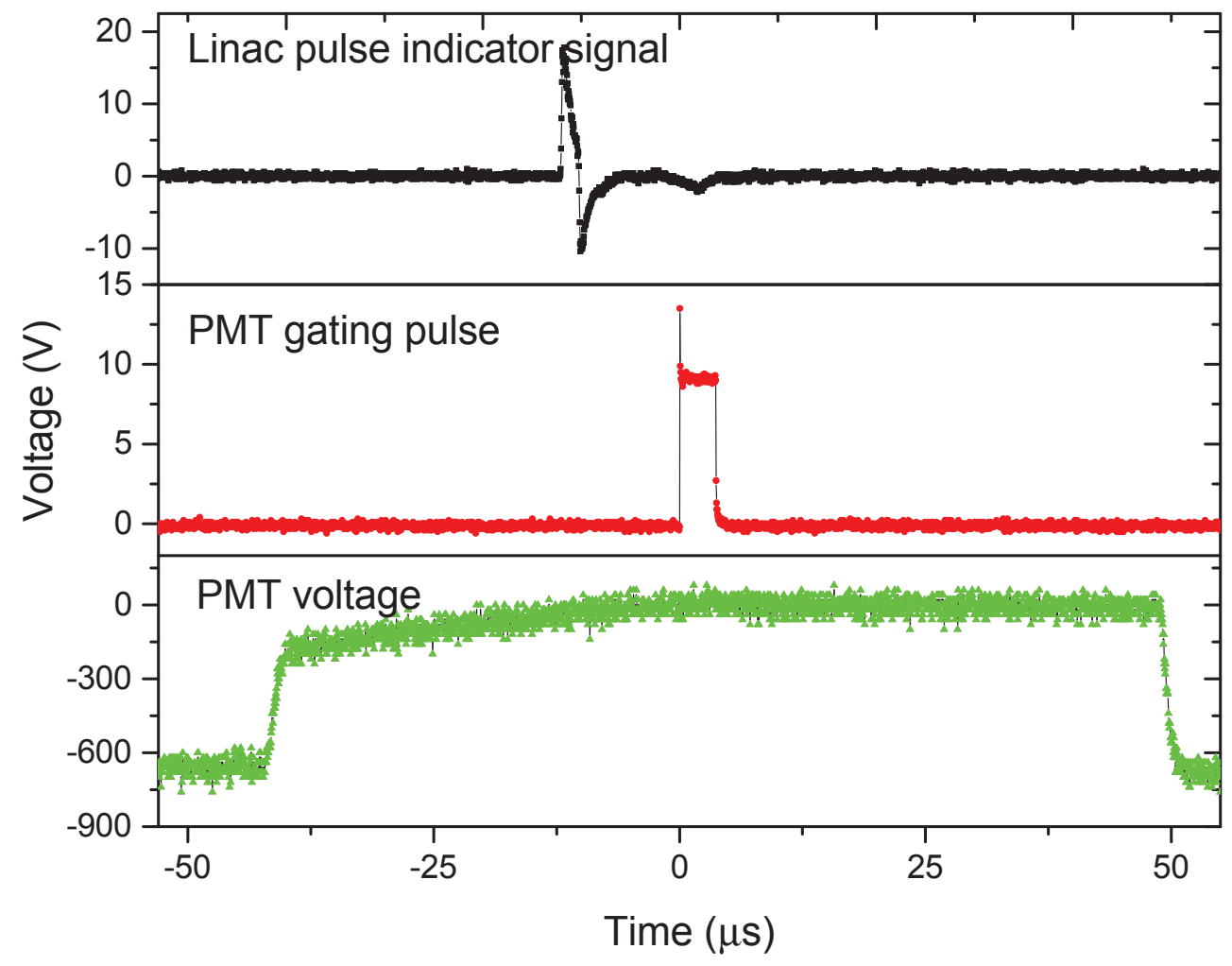

Figure 3.12: Sample oscilloscope traces illustrating the gating of the PeTrack detectors during linac operation. Linac pulse indicator signal: this pulse leads every $\mathrm{x}$ ray pulse; it is generated by the PFN and starts before actual x rays are produced. PMT gating pulse: the linac pulse indicator signal triggers a signal generator to produce a square PMT gating pulse. PMT voltage: the PLL synchronizes with the linac pulse indicator signal and generates a high voltage gating window $90 \mu$ s wide, roughly centred on the PMT gating pulse. 


\subsubsection{Phantom motion, fiducial marker, and beam delivery}

An anthropomorphic dynamic thorax phantom (Dynamic Thorax Phantom Model 008A, CIRS Inc., Norfold, VA) simulated a patient's thorax and breathing motion. The phantom accurately provides known and repeatable 3D motion of a target inside an anthropomorphic thorax made from tissue equivalent epoxy materials. A rod composed of lung tissue equivalent material is inserted in the phantom and connects to a motion actuator box. The rod rotates around its central axis in the anterior-posterior (AP) and left-right (LR) directions and translates in the inferior-superior (IS) direction. The rod has an insert which can hold radiochromic film. Gafchromic®EBT3 radiochromic films (Ashland Inc., Covington, KY) were placed in the rod insert. The rod was oriented so that the films were parallel to the coronal plane and approximately at the isocenter of the linac. A single ${ }^{22} \mathrm{Na}$ fiducial marker was placed in a small insert along the axis of the rod (see Figure 3.13). The activity of the marker on the day of the measurements was $0.43 \mathrm{MBq}(11.6 \mu \mathrm{Ci})$. Figure 3.7 shows the setup of the detector system and phantom in relation to the linac.

The motion of the rod of the phantom is controlled by the Motion Control software. Two modes of motion were used to translate the rod in the IS direction with amplitude $\pm 10 \mathrm{~mm}$. The first mode was based on a sinusoidal asymmetric breathing motion model [77], parametrized specifically as:

$$
y(t)=A\left(2 \cos ^{4}\left(\frac{\pi t}{T}-\phi\right)-1\right)+y_{0}
$$

where $A$ is the amplitude of the centroid of the rod, $y_{0}$ is the initial offset, $T$ is the period, and $\phi$ is the phase at the start of the breathing cycle. The range of motion is $\left[y_{0}-A, y_{0}+A\right]$. Real patient breathing motion is noisy, irregular, and exhibits inter- and intracycle variability in amplitude and period. [78-81] The second mode of motion was based on real patient breathing motion measured with an electromagnetic 


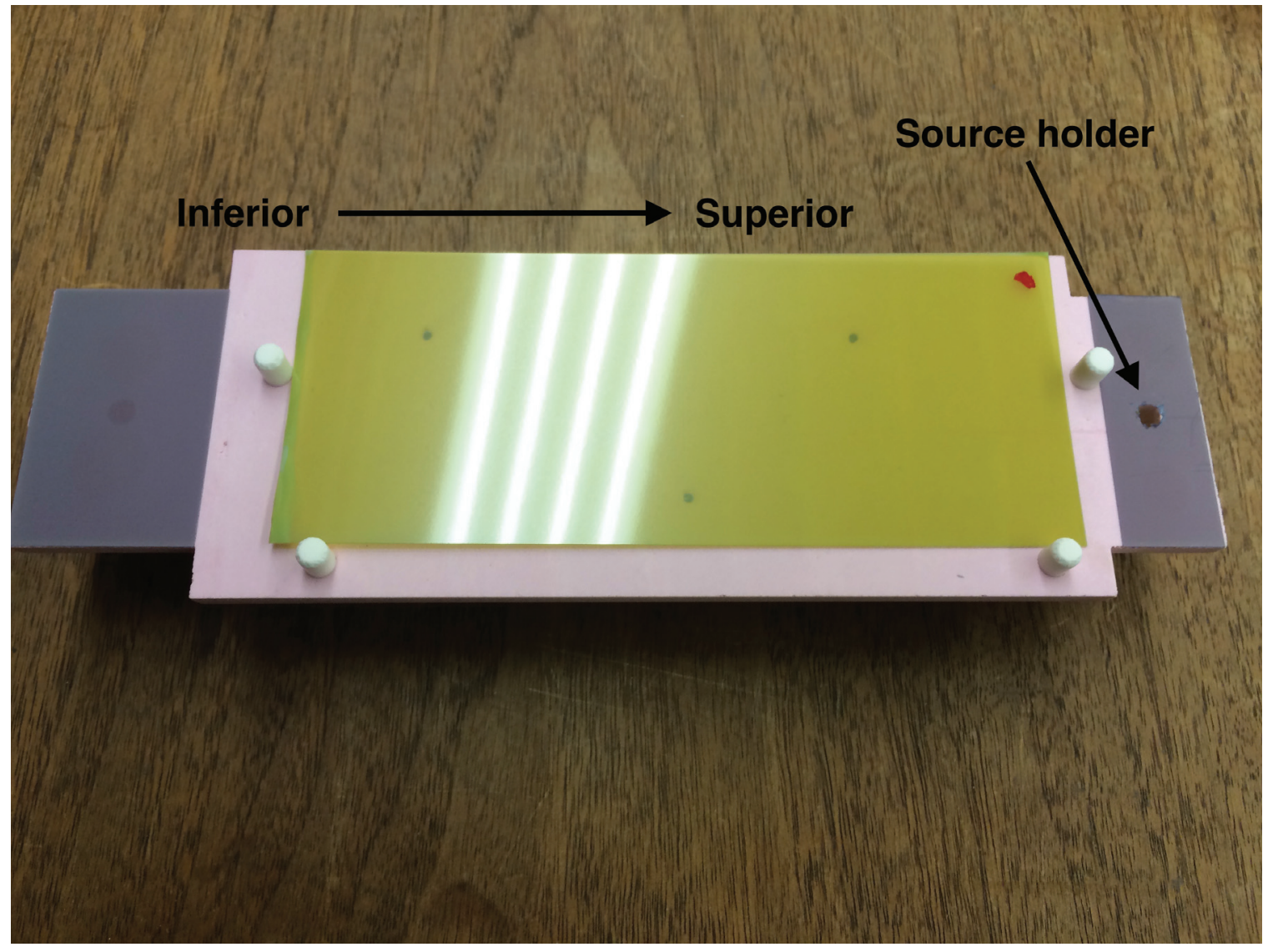

Figure 3.13: Radiochromic films were placed within the rod and marked for registration. A small fiducial marker was placed at the superior end of the rod, in a source holder. 
Table 3.1: Parameters of each beam delivery.

\begin{tabular}{ccccc}
\hline \hline Delivery \# & Type & Motion & $\begin{array}{c}\text { Beam energy } \\
(\text { MV })\end{array}$ & $\begin{array}{c}\text { MU delivered } \\
\text { (actual) }\end{array}$ \\
\hline 1 & Non-gated & Static & 6 & 492.4 \\
2 & Non-gated & Sinusoidal & 6 & 492.4 \\
3 & Gated & Sinusoidal & 6 & 492.4 \\
4 & Non-gated & Patient breathing & 6 & 492.4 \\
5 & Gated & Patient breathing & 6 & 492.4 \\
6 & Non-gated & Patient breathing & 10 & 495.6 \\
7 & Gated & Patient breathing & 10 & 494.7 \\
\hline \hline
\end{tabular}

tracking device placed on the surface of the abdomen of the patient [82]. The period was $60 \mathrm{~s}$ for both modes of motion. This choice is better explained in the context of the latency of the system (see Section 3.3.4). A one dimensional gating window of size $5 \mathrm{~mm}$ in the IS direction roughly centered on the exhale phase of the breathing cycle was used.

The phantom was irradiated with a photon beam of size $3 \times 3 \mathrm{~cm}^{2}$ at isocenter. Each delivery was set to deliver 500 monitor units (MU) at a rate of $125 \mathrm{MU} / \mathrm{minute}$. Seven film measurements were done. A reference dose distribution in the absence of motion was measured by irradiating the phantom while immobile. A combination of non-gated and gated deliveries was done for the two modes of motion and for nominal beam energies of 6 and 10 MV. Table 3.1 summarizes the parameters of each delivery.

\subsubsection{Latency of the system}

Real time tracking for respiratory gated radiotherapy is complicated by the inherent latency of the system. 
From rough estimates, it takes almost four seconds for the beam of the NRC Elekta Precise to turn on from its off state. By comparison, turning the beam off is much faster $(<1 \mathrm{~s})$. The beam-on delay can be reduced to $<2 \mathrm{~s}$ by preventing the current of the filament of the electron gun from dropping to its standby value when holding the beam off. Since typical breathing motion has a period of 4 to $8 \mathrm{~s}[78,83]$, a beam-on delay of $2 \mathrm{~s}$ would still be too slow to deliver a respiratory gated treatment clinically. However, it has been shown that the beam-on delay can be reduced to less than $0.22 \mathrm{~s}$ on an Elekta linac with the use of the Elekta Response ${ }^{\mathrm{TM}}$ gating interface. [84, 85] A certain group reported time delays of $850 \mathrm{~ms}$ when using the Response interface. [86] However they did not attempt to prevent the gun current from dropping to its standby value, which was recommended by Cui et al. [84]. ${ }^{6}$ The Response interface only requires an Elekta linac with a fast tuning magnetron [87] and a linac control system with Desktop version 6.0 or higher. The interface connects directly to the PRF enable chain of the Elekta linac and controls the physical relays which allows to pause and resume beam delivery. This makes the Response interface simple and straightforward to install to existing Elekta linacs.

In this proof-of-concept study without access to the Response gating interface, the period of the breathing motion and the localization frequency were scaled by a factor of 10 to account for the $2 \mathrm{~s}$ beam-on delay on the NRC linac. The breathing period was scaled to $60 \mathrm{~s}$ for the sinusoidal motion. The patient breathing dataset exhibited variability in its period: it had an average breathing period of $5.9 \pm 0.8 \mathrm{~s}$ over 17 breath cycles. The patient breathing data were also scaled by a factor of 10 in time when it was imported in the Motion Control software of the phantom, i.e. the average breathing period was scaled to $59 \pm 8 \mathrm{~s}$. The marker was located every $2 \mathrm{~s}$. The past 30 locations of the marker (corresponding to one breathing cycle) were

\footnotetext{
${ }^{6}$ In fact, Elekta itself recommended the gun current modification to us in the course of this study and we found that this simple fix significantly shortened the beam on delay.
} 
used by the nLMS filter to predict the location of the marker $2 \mathrm{~s}$ ahead. The gating of the linac beam was based on the predicted location of the marker to account for the system latency. The current from the ion chamber placed within the beam at the exit of the phantom was recorded to assess and verify synchronization of the gating with the tracking and calculate the duty cycle. The duty cycle is defined as:

$$
\text { Duty cycle }=\frac{(\text { total beam on time })}{(\text { total treatment time })} \times 100 \%
$$

\subsubsection{Results}

\section{Synchronization of gating with tracking}

Figures 3.14 and 3.15 demonstrate the synchronization of the tracking of the motion of the rod with the gating of the beam. The dashed area represents the gating window: the beam is delivered when the marker is located within this region. Gated deliveries $\# 3$, \#5, and \#7 had duty cycles of $43 \%, 36 \%$, and $36 \%$ respectively. Note that during delivery \#3 (see Figure 3.14), the beam inadvertently turned off for a few seconds approximately 6 minutes into the delivery due to an operator mistake. The beam was promptly turned back on. The effect of this unexpected event should be negligible given the short time the beam was off compared to the total duration of the delivery. During deliveries \#5 and \#7, the marker sometimes moved in, out, and back into the gating window in rapid succession (for example, at approximately the 7-minute mark on Figure 3.15 a)). This in turn causes the beam to rapidly turn on, off, and on, in accordance with the design of the gating system.

Figure 3.16 gives a close look on the synchronisation of the gating with PeTrack. In this figure, the predicted location of the marker has been shifted by one localization interval. In other words, at any given time $t_{n}$, the current curve gives the current location of the marker whereas the predicted curve gives the predicted location of the 


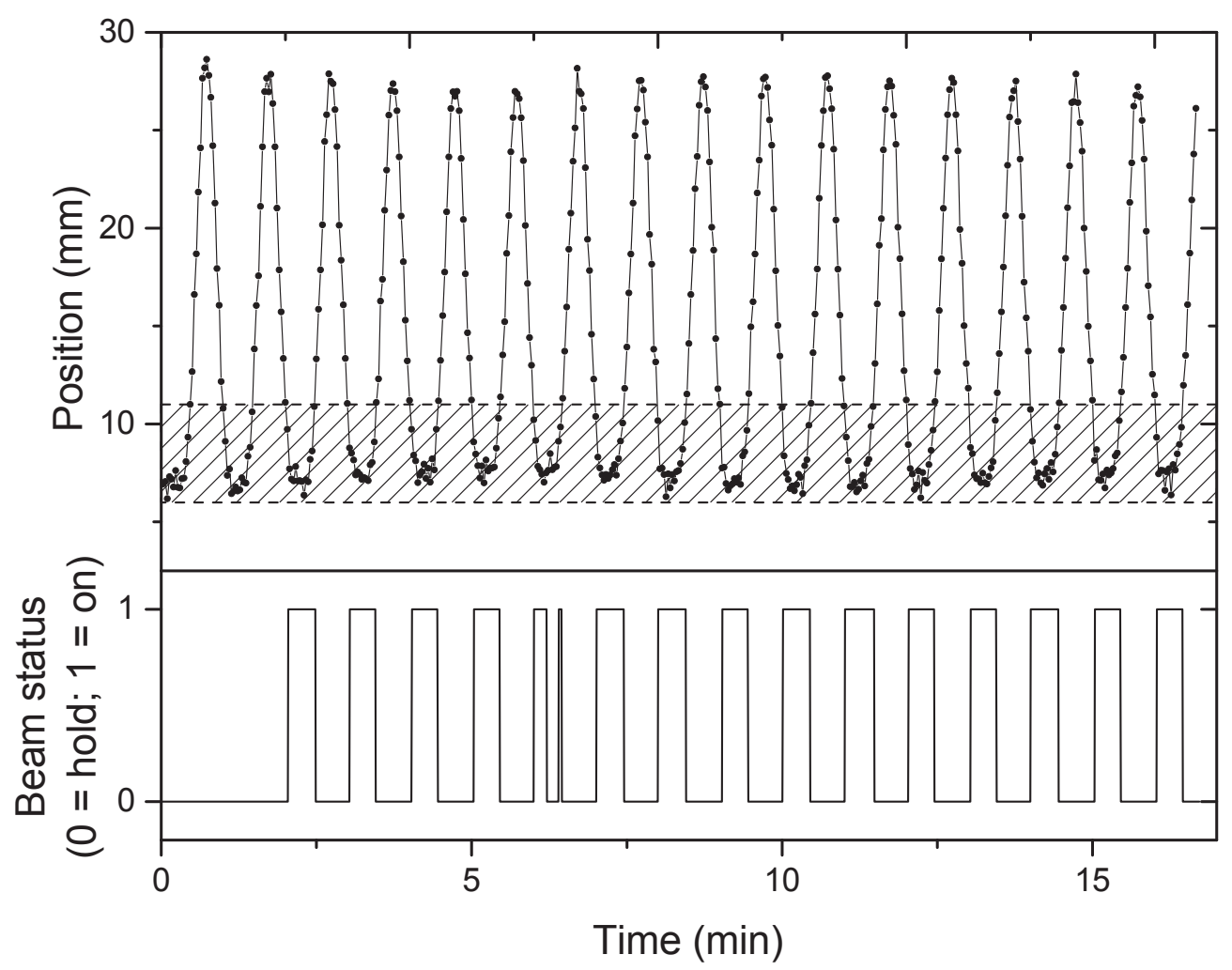

Figure 3.14: Position of the marker in the IS direction (top) and status of the beam (hold or on; bottom) throughout the delivery with sinusoidal translation of the rod. The dashed area represents the gating window within which the beam is on. Note that approximately 6 minutes into the delivery of the beam, the beam inadvertently turned off due to an operator mistake. The beam was promptly turned back on. 


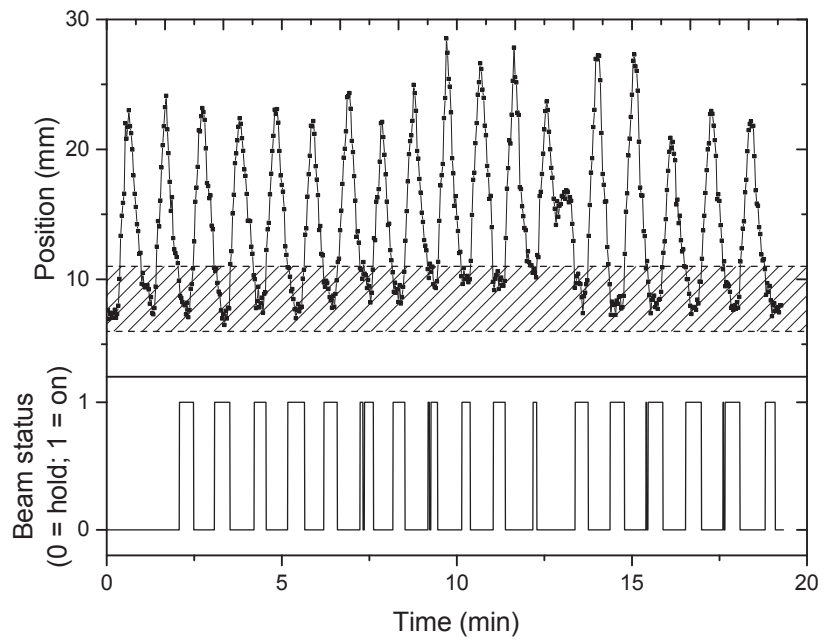

(a) $6 \mathrm{MV}$

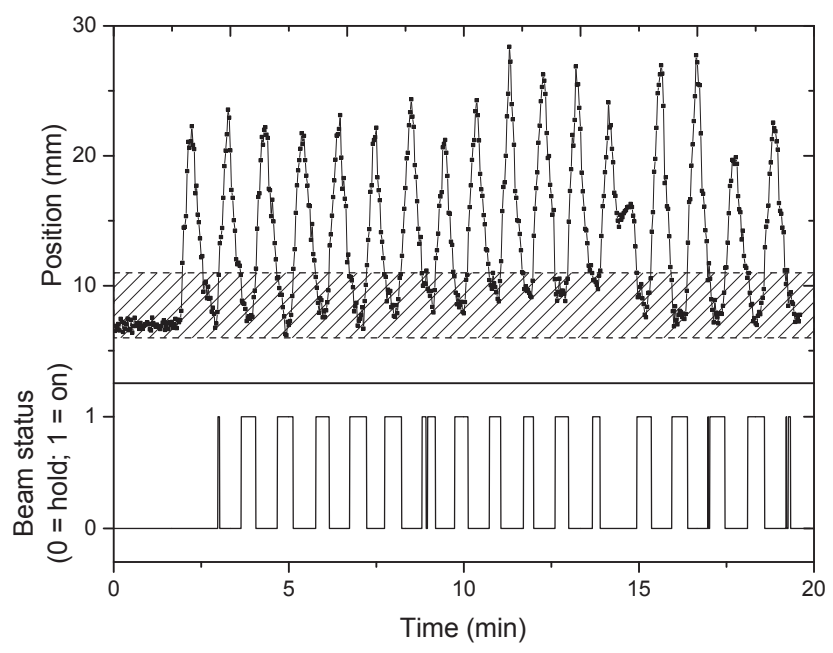

(b) $10 \mathrm{MV}$

Figure 3.15: Position of the marker in the IS direction and status of the beam (hold or on) throughout the deliveries with real patient breathing motion. The dashed area represents the gating window within which the beam is on. Note that at various moments (for example, at around 7 minutes on subfigure a)), the marker moves out of the gating window soon after first entering it, then subsequently re-enters the gating window. This causes the beam to turn on, off, and on again in quick succession, in accordance with the design of the gating system. 
Table 3.2: FWHM of profiles for each delivery.

\begin{tabular}{cccc}
\hline \hline Delivery \# & $\begin{array}{c}\text { FWHM } \\
(\mathrm{mm})\end{array}$ & $\begin{array}{c}\text { Change from reference } \\
(\%)\end{array}$ & $\begin{array}{c}\text { Absolute change } \\
\text { from reference }(\mathrm{mm})\end{array}$ \\
\hline 1 (static reference) & 32 & N/A & N/A \\
2 & 42 & 31 & 10 \\
3 & 34 & 6 & 2 \\
4 & 40 & 25 & 8 \\
5 & 34 & 6 & 8 \\
6 & 40 & 25 & 3 \\
7 & 35 & 9 & 2 \\
\hline \hline
\end{tabular}

marker at time $t_{n+1}$, based on the current location and 29 previous locations of the marker before $t_{n}$. The gating interface sends the beam on signal when the predicted location of the marker is within the gating window; similarly the beam off signal is sent when the predicted location of the marker is outside the gating window.

\section{Radiochromic films}

The radiochromic films were scanned approximately 48 hours after exposure. Figures 3.17 and 3.18 demonstrate the effect of respiratory gating on the dose distribution in the presence of sinusoidal motion and real patient breathing motion respectively. Figures 3.19 and 3.20 show line profiles (indicated by arrows on Figures 3.19 and 3.18) measured in the IS direction for each dose distribution. Individual profiles were normalized to the maximum of the profile of the static reference after subtracting their own background. Table 3.2 lists the full width at half maximum (FWHM) for each profile. 


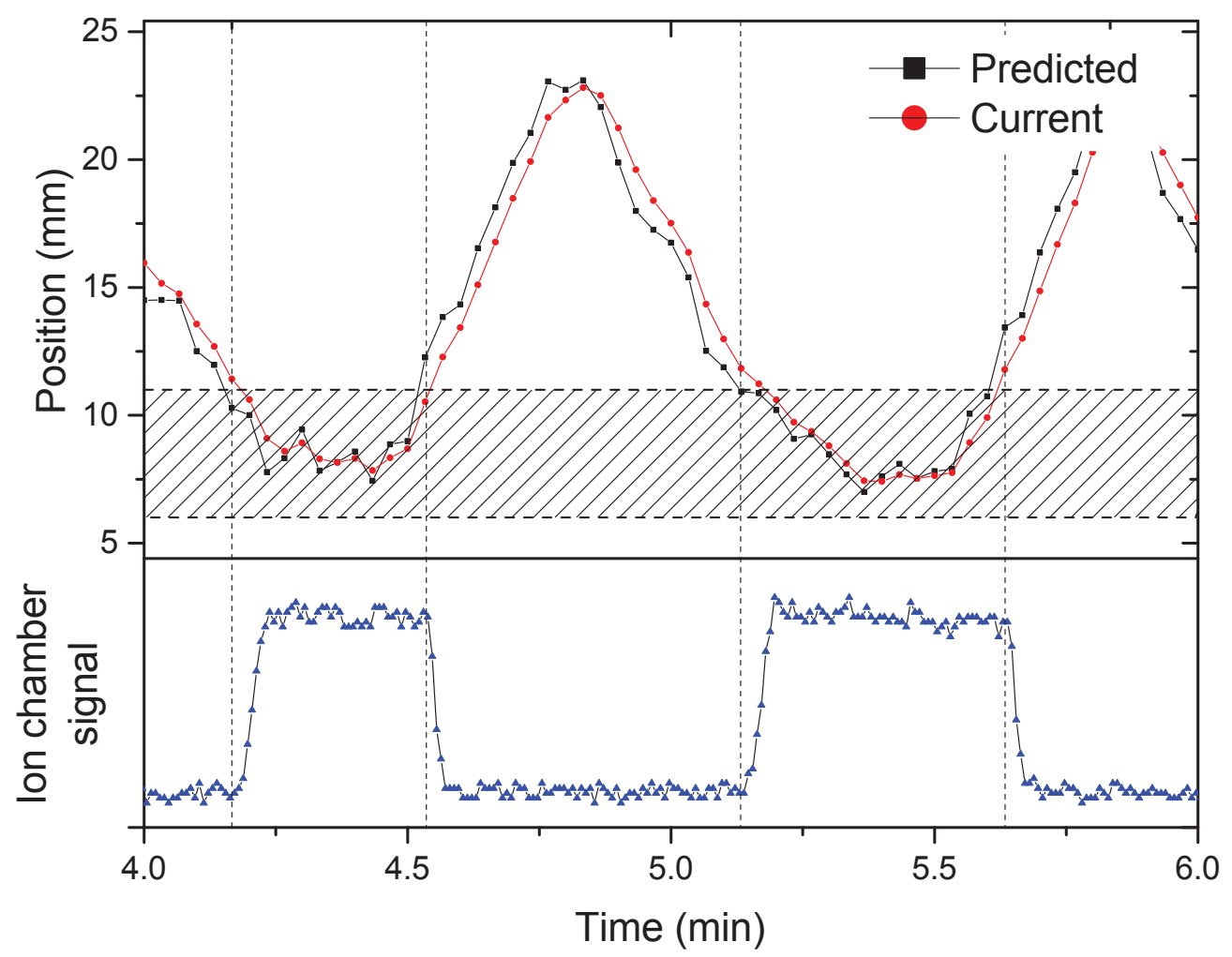

Figure 3.16: Predicted and current position of the marker at this time (top) plotted with the current in the ion chamber (bottom). The dashed area represents the gating window. Note that the predicted location of the marker has been shifted by one localization interval. In other words, at any given time $t_{n}$, the current curve gives the current location of the marker whereas the predicted curve gives the predicted location of the marker at time $t_{n+1}$, based on the current location and 29 previous locations of the marker before $t_{n}$. 


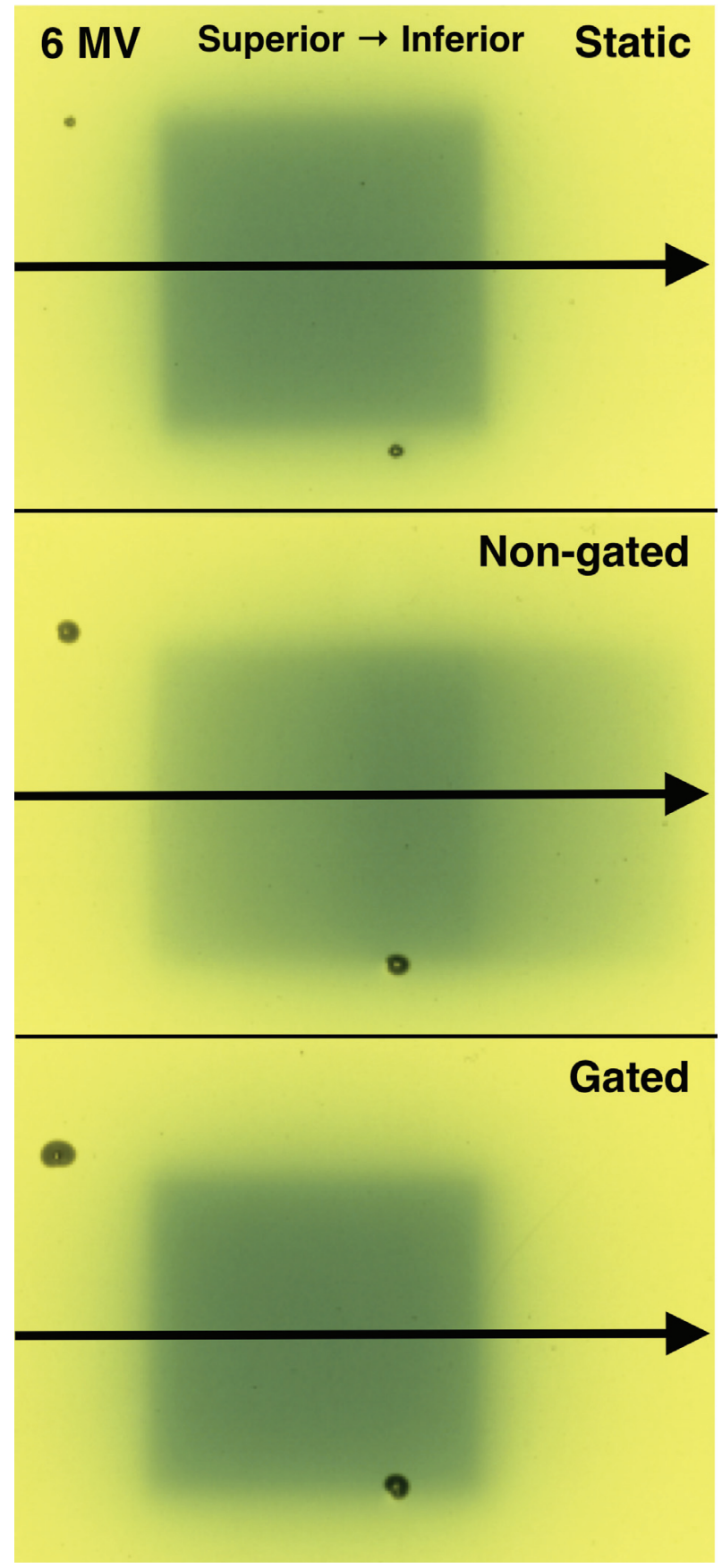

Figure 3.17: Radiochromic films after exposure to $500 \mathrm{MU}$ of a $3 \times 3 \mathrm{~cm}^{2}$ photon beam at nominal energy $6 \mathrm{MV}$. The phantom rod was translated sinusoidally in the IS direction. Line profiles in the IS direction (indicated by the arrows) were measured and plotted in Figure 3.19. 


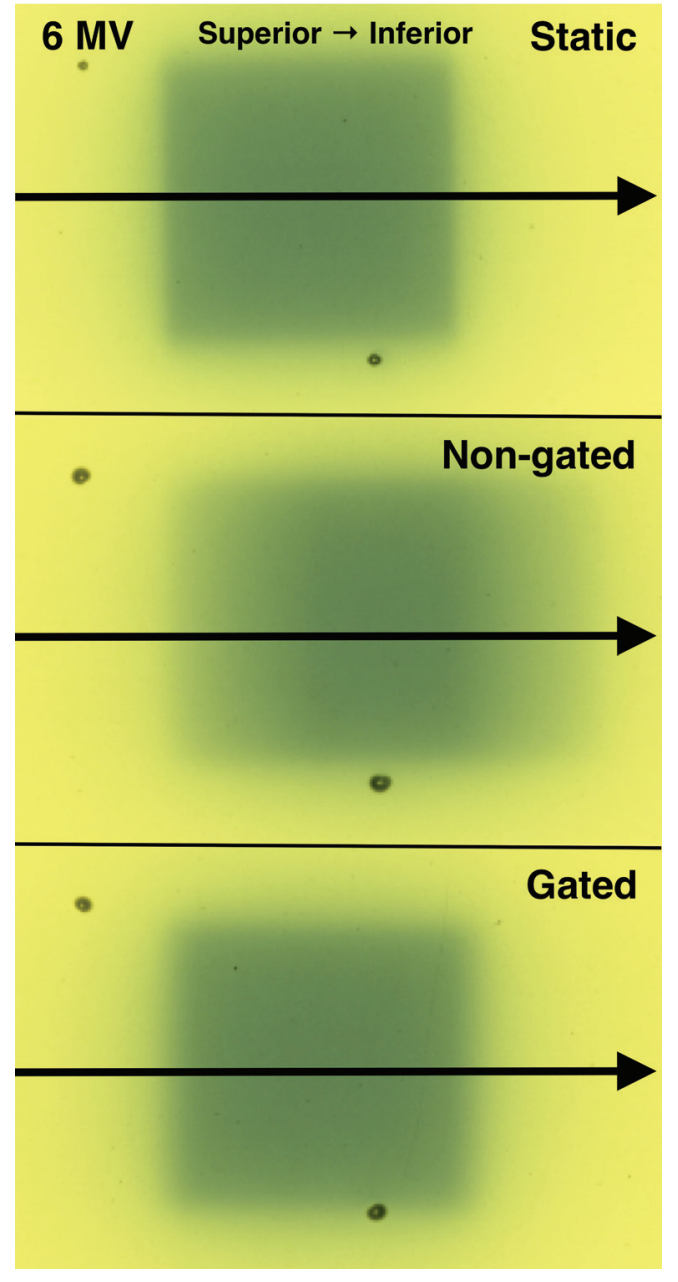

(a) $6 \mathrm{MV}$

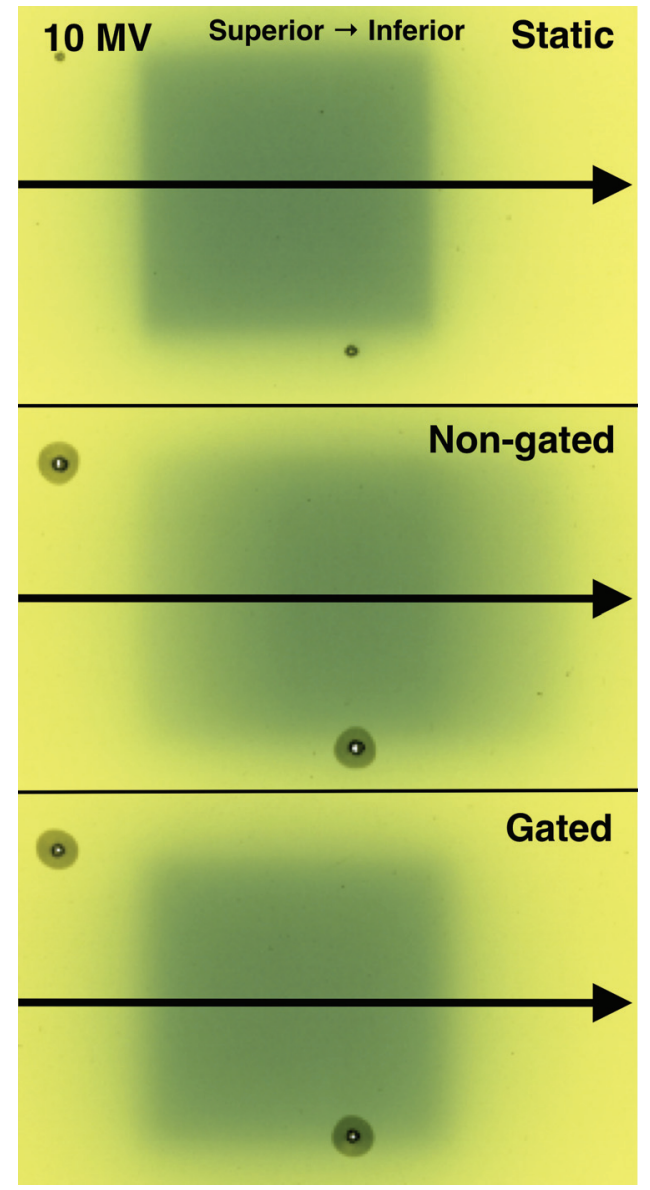

(b) $10 \mathrm{MV}$

Figure 3.18: Radiochromic films after exposure to $500 \mathrm{MU}$ of a $3 \times 3 \mathrm{~cm}^{2}$ photon beam at nominal energies 6 and $10 \mathrm{MV}$. The translation of the phantom rod in the IS direction was based on real patient breathing data. Line profiles in the IS direction (indicated by the arrows) were measured and plotted in Figure 3.20. 


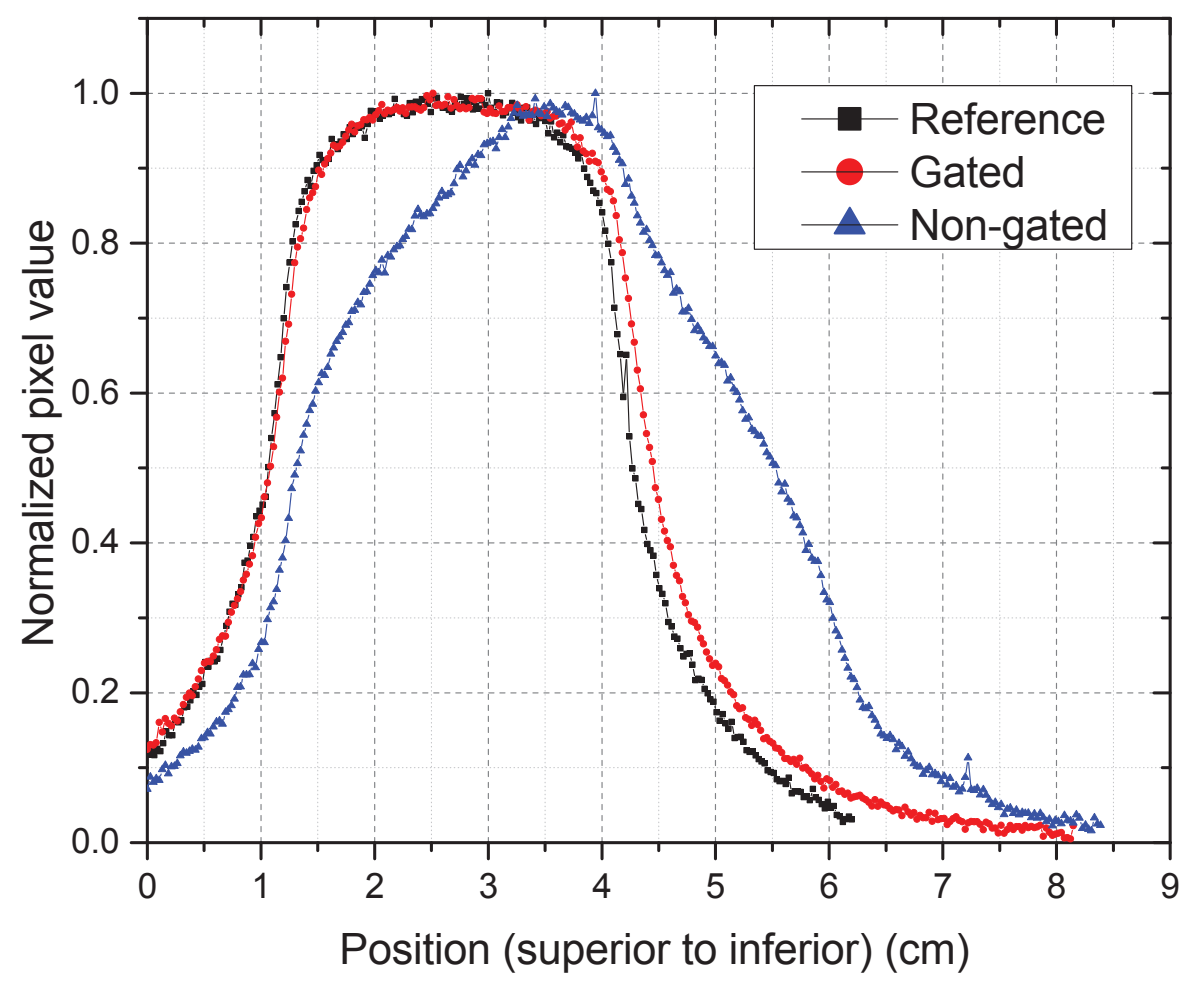

Figure 3.19: Line profiles across the dose distribution in the IS direction for films exposed under sinusoidal motion. The values are normalized to the maximum of the reference profile, after subtracting their individual background. 


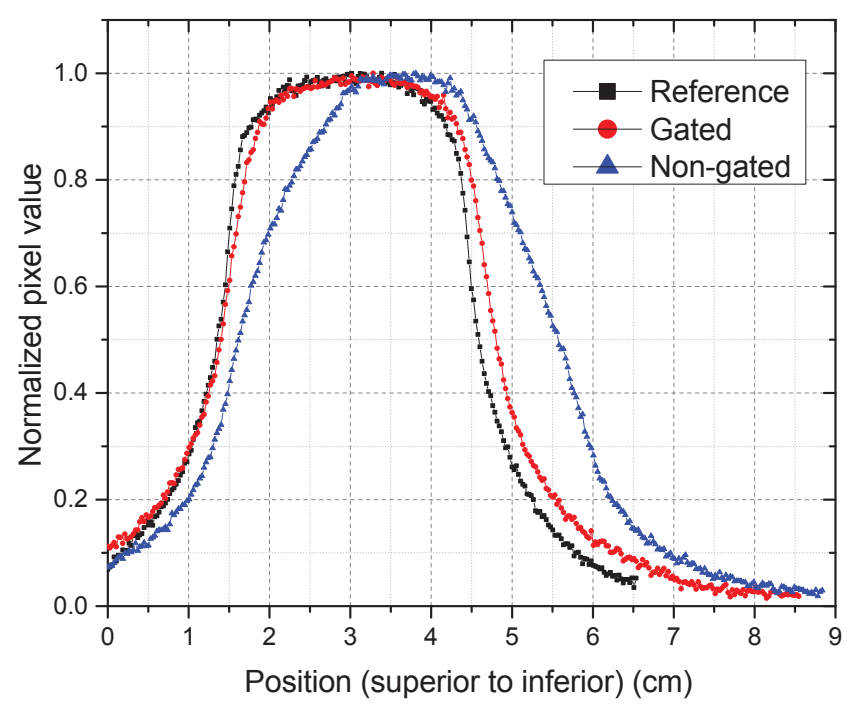

(a) $6 \mathrm{MV}$

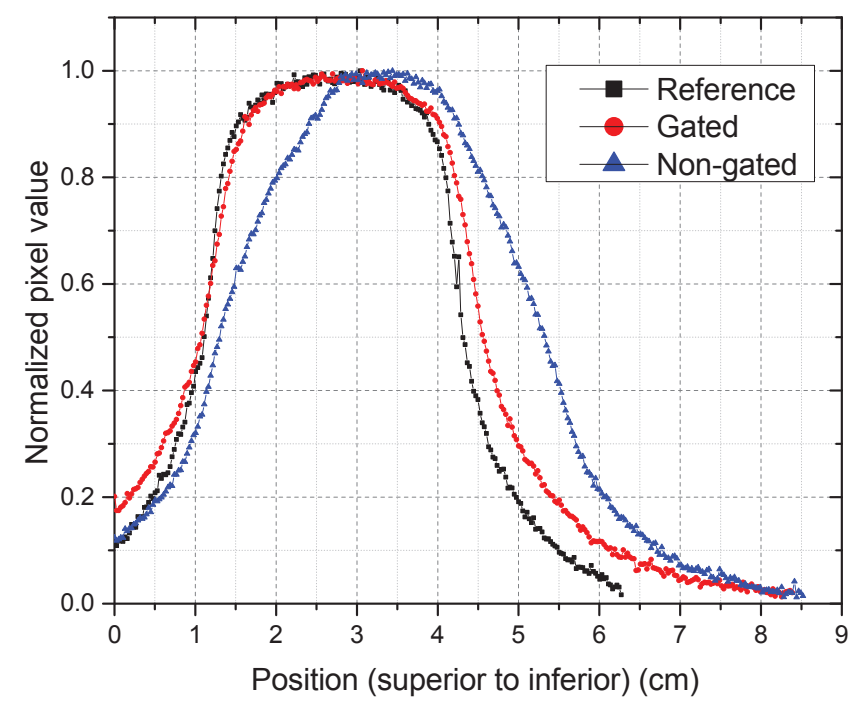

(b) $10 \mathrm{MV}$

Figure 3.20: Line profiles across the dose distribution in the IS direction for films exposed under real patient breathing motion. The values are normalized to the maximum of the reference profile, after subtracting their individual background 


\subsection{Discussion}

\subsubsection{EGSnrc simulation}

Kozma et al. [88] found that radiation damage to individual BGO crystals is negligible below $10^{4}$ Gy and only significant at doses of $10^{5}$ Gy. Furthermore complete recovery from the damage is achieved after a few days of no exposure. Results from the EGS++ simulations clearly show that the dose to the BGO will be much smaller than those thresholds. Thus radiation damage from the scattered radiation will be negligible for the PeTrack detectors.

\subsubsection{Respiratory gating}

Figures 3.17 and 3.18 clearly show the effect of gating on the dose distribution: the square shape of the field is mostly preserved compared to the blurred distribution of the non-gated delivery. This is also confirmed by the line profiles through the films shown in Figures 3.19 and 3.20. The FWHM of the profiles of the gated deliveries are 2 to $3 \mathrm{~mm}$ larger than the reference due to the $5 \mathrm{~mm}$ gating window and to time delays. There are also uncertainties introduced by the co-registration of the films. This process involved physically marking the films at three different locations with a needle inserted through small openings in the rod. After the films were scanned, the images of the films were manually aligned in an image processing software (Pixelmator, The Pixelmator Team, Vilnius, Lithuania). The overall uncertainty of this co-registration method is approximately $\pm 1.1 \mathrm{~mm}$ in both the IS and LR directions. However, this uncertainty should not affect the result of the comparison of the FWHM.

The line profiles are presented as pixel values normalized to the static reference because the experiment was done as a proof of concept on a research linac. Weibert et al. suggest that the dosimetry (which varies between individual linacs) should 
be carefully assessed before introducing a new respiratory gating system into clinical use. [89] An absolute dosimetric evaluation is thus better left to the commissioning of PeTrack-based gating at the clinic level.

The results from the gating experiment demonstrates that the dose distribution is better confined to the target volume when using PeTrack for respiratory gating. This means that the target volume will receive a higher dose with the use of gating than without, hopefully resulting in better outcomes for the patients. Similarly, normal tissues will receive a lower dose with the use of gating than without, which will potentially contribute to lower normal tissue complications.

Figure 3.15 reveals a common feature of patient breathing patterns: a baseline shift. The shift in the baseline causes gates to vary in length. Clinically such a shift would be observed in real time by the operator (or potentially detected automatically by the tracking software). Corrective actions could be taken such as shifting the gating window according to the baseline shift.

The effects of timing delays can be seen in Figure 3.16. The first beam delivery on Figure 3.16 shows the beam on delay of approximately $2 \mathrm{~s}$. This delay causes the marker to already be within the gating window when the beam comes on, decreasing the duty cycle. On the other side of the gating window, the monitor chamber signal demonstrates the faster beam off time, but it is still possible for the marker to move out of the gating window by the time the beam comes off. This would result in increased dose to surrounding normal tissues. The magnitude of these effects depend on several factors: the value of the time delays, the speed of the marker, the prediction error of the adaptive filter, etc. Having a large duty cycle will reduce the relative magnitude of the effects, but at the expense of larger margins. Smith et al. have studied the interplay of the time delays in a particular gating system with the delivered dose distribution. [90] Time delays can cause significant systematic errors in treatment. They propose that such delays be taken into account during treatment 
planning by use of an additional margin to the internal treatment volume (ITV) so that the target remains within the beam during at least $95 \%$ of the beam on time. Modifying van Herk's formula for margins [91], the additional margin due to time delays alone is:

$$
M_{t_{d}} \approx v_{d} \times\left|t_{d}-\left(t_{a v g} \times 5 \%\right)\right|+2.5 \Sigma+0.7 \sigma
$$

where $v_{d}$ is the average speed of the target, $t_{d}$ is the time delay in the system, $t_{a v g}$ is the average beam on time per breathing cycle, $\Sigma$ is the standard deviation of systematic errors, and $\sigma$ is the standard deviation of random errors. Errors such as patient setup, tumour delineation, etc., would be included as additional values in the second and third term of the equation above.

Tumour motion displays great variability in its characteristics. Shirato et al. have measured lung tumour speeds by imaging implanted fiducial markers and reported a median speed of $10 \pm 5 \mathrm{~mm} / \mathrm{s}$. [92] In addition, PeTrack actually reports a time-averaged location along the motion track of the marker between two successive localizations. [2] This contributes a small systematic error which depends on the speed of the target. Let's assume the beam on delay of the linac can be decreased to $0.2 \mathrm{~s}$ (as posited in Section 3.3.4) and that PeTrack locates the marker every 0.2 s. Using the median tumour speed from Shirato et al., the margin needed to account for time delays alone is approximately $4.4 \mathrm{~mm}$, including the random error from the standard deviation of the prediction error of the nLMS filter $(0.9 \mathrm{~mm}$ at a latency of $0.2 \mathrm{~s})$.

\subsection{Conclusion}

This chapter presented an evaluation of respiratory gated radiotherapy using PeTrack. The delivered dose distribution of gated and non-gated deliveries were compared and showed that PeTrack-based respiratory gating can greatly reduce the blurring of the 
dose profiles due to motion. The next chapters will cover the various applications of PeTrack in PET imaging. 


\section{Chapter 4}

\section{Motion tracking in PET imaging using PeTrack}

\subsection{Introduction}

Section 1.2 already motivated the need for motion tracking in PET imaging. PET imaging currently uses several of the same motion tracking techniques as radiotherapy. Spirometry, pressure belts, and optical markers are all used in PET imaging to detect motion (see Section 3.1). Again, the Varian RPM system is the most commonly used motion detection tool for respiratory gated PET imaging.

There is also a variety of data driven techniques to extract motion information. For example, a region of interest (ROI) is defined around the boundaries of moving organs with different tracer activity uptake. As the organs move, the number of counts within the ROI varies with time and is used to determine a respiratory signal. [93] A similar approach uses spectral analysis directly on the sinogram data to detect motion. [94] The centre of mass of LORs in sinogram space can also be used to track motion of certain structures in the axial direction. [95-97] Büther et al. [97] also make use of the variation in axial sensitivity of PET scanners. Motion information 
is obtained from Fourier analysis of the number of counts in each axial slice as a function of time.

Other methods involve the use of external positron sources. For example, the position of fiducial positron emission markers can be determined from reconstructed PET images. If the PET images are reconstructed in short time frames (e.g. 0.5 or $1 \mathrm{~s}$ ), the motion trace of the markers can be obtained retrospectively. [98, 99] To have sufficient signal to noise ratio this requires markers with individual activities of $500 \mathrm{kBq}$ when the phantom activity is $70 \mathrm{MBq}$.

Büther et al. [100] developed a data-driven method that uses external fiducial positron emission markers for respiratory gating in PET. Their approach involves identifying the markers on a reconstructed image and forward projecting volumes of interest centered on the markers. A surrogate signal related to the vertical motion of external markers can be calculated from the resulting sinograms.

It has also been proposed to use the Kinect system (Microsoft Corporation, Seattle, WA) to track body motion during PET imaging. [101] The Kinect is a low-cost handheld 3D scanner available commercially. A speckle pattern of infrared light is projected onto a surface. The Kinect infers depth information from the way the surface distorts the speckle pattern. This method has high resolution and can track the 3D surface of the patient body. The system is sensitive enough to pick up the heart rate from the vibrations of the patient's chest during breath holds. Drawbacks include the extra hardware and coregistration with the scanner required.

\subsubsection{PeTrack for motion tracking in PET imaging}

The PeTrack tracking algorithm can be used to track 3D motion in PET imaging using raw list mode data and an external fiducial marker. If a low activity external fiducial marker is placed on a patient's abdomen during a PET scan, the PET scanner will record the coincidence events from the marker in addition to the events from 
the physiological tracer activity within the patient. Low activity markers on the order of a few microCurie activity have been shown to create no obvious artefacts on reconstructed PET images. [100] The PeTrack tracking algorithm can process raw PET data in list mode format and track the location of the marker during the scan at a certain sampling frequency. In this specific application, no hardware other than the PET camera and an external positron emission fiducial marker is required. In addition, there is no need to bin the list mode into sinograms or to reconstruct images, both of which can be quite lengthy due to the large number of recorded events during PET acquisition.

The motion of the marker on the patient's abdomen provides a surrogate for the motion of the patient's body. The motion can include gross patient body motion (e.g. if the patient coughs or moves substantially during the scan) and respiratory motion (including baseline shift in the breathing pattern). This motion information can be used for retrospective respiratory gating or motion correction, both of which will be explored in the next chapter. PeTrack can provide this motion information by tracking the marker at a sampling frequency of $2 \mathrm{~Hz}$ (i.e. localizing the marker every $0.5 \mathrm{~s}$ ), which is sufficient to resolve respiratory motion, as will be shown below.

This chapter covers the modifications required to use PeTrack for motion tracking in PET imaging. The performance of the modified tracking algorithm was evaluated using both simulation and phantom studies.

\subsection{The background problem}

One problem presents itself to using PeTrack for motion tracking during PET imaging: the very high physiological tracer background activity within the patient. The total injected activity of tracer can be higher than $555 \mathrm{MBq}(15 \mathrm{mCi})$, but the PeTrack fiducial marker has relatively low activity $(\sim 2.5 \mu \mathrm{Ci})$. 


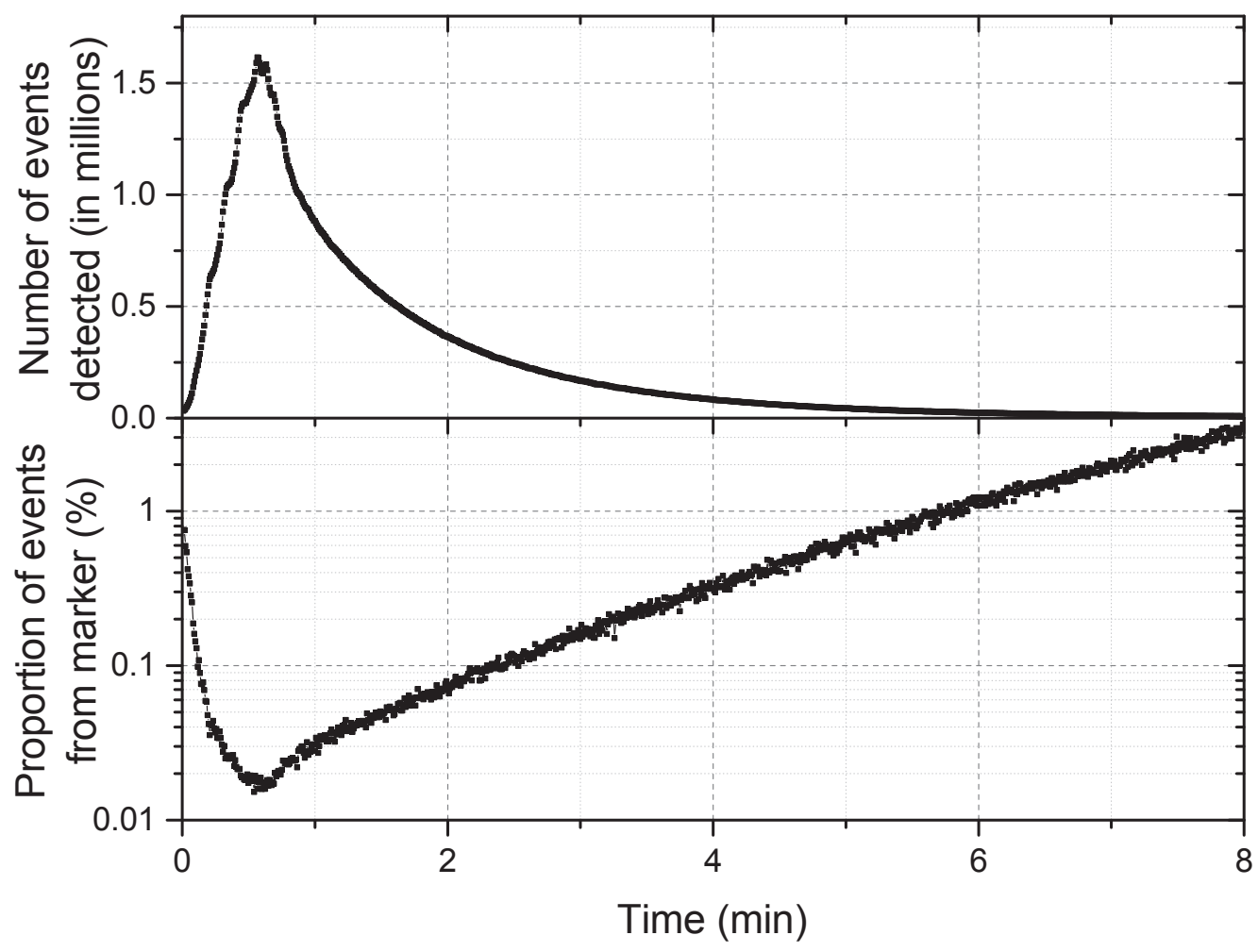

Figure 4.1: Top: Number of events detected in $0.5 \mathrm{~s}$ bins during a dynamic cardiac PET scan. The patient was injected with $840 \mathrm{MBq}$ of ${ }^{82} \mathrm{Rb}$. Bottom: Proportion of the total detected events (in $0.5 \mathrm{~s}$ bins, since PeTrack will track the marker every $0.5 \mathrm{~s}$ ) that came from a single $2.5 \mu \mathrm{Ci}$ marker placed on the patient's abdomen.

The top half of Figure 4.1 shows a sample time activity curve (TAC, i.e. the total counts recorded by the scanner as a function of time) for a patient undergoing dynamic cardiac PET imaging. The patient was initially injected with $840 \mathrm{MBq}(22.7 \mathrm{mCi})$ of ${ }^{82} \mathrm{Rb}$. The bottom half of Figure 4.1 shows the proportion of the detected events that came from a $2.5 \mu \mathrm{Ci}$ marker placed on the patient's abdomen.

At the peak of the TAC, the events from the marker only account for $0.01 \%$ of the total activity recorded by the scanner. Once the tracer has significantly decayed, the marker still only accounts for less than $5 \%$ of the total activity recorded. This 
highlights that the signal from the PeTrack marker is extremely weak in comparison to the background from the patient.

This causes one of two effects. If the automatic initialization of the algorithm is used, then it returns a location near the centre of mass of the activity distribution within the patient. Even when a good estimate of the marker's initial location is used, the current implementation of the EM algorithm still fails to converge due to the overwhelming background. The solution is to avoid using the automatic initialization of the algorithm and to include background rejection techniques to the EM algorithm.

\subsection{Modified initialization of the algorithm}

The algorithm is initialized by manually providing it with an estimate of the marker's initial location. The initial location can be estimated in one of two ways:

1. When a dual PET/CT system is used, CT images of the patient are typically acquired before a PET scan for the purpose of attenuation correction during image reconstruction. The fiducial marker used by PeTrack is visible on those images (see Figure 4.2). The location of the marker on the CT images can be used as the estimate of the marker's initial location.

2. If CT images are not available, the raw PET data acquired during the first $30 \mathrm{~s}$ of the scan can be reconstructed. The marker will be visible on the reconstructed image and its location can be used to initialize the PeTrack algorithm (see Figure 4.3).

The automatic initialization method presented in Section 2.2.3 could also be used if we allow an initialization period before injection of the PET tracers. However, this may require changing patient imaging protocols. 


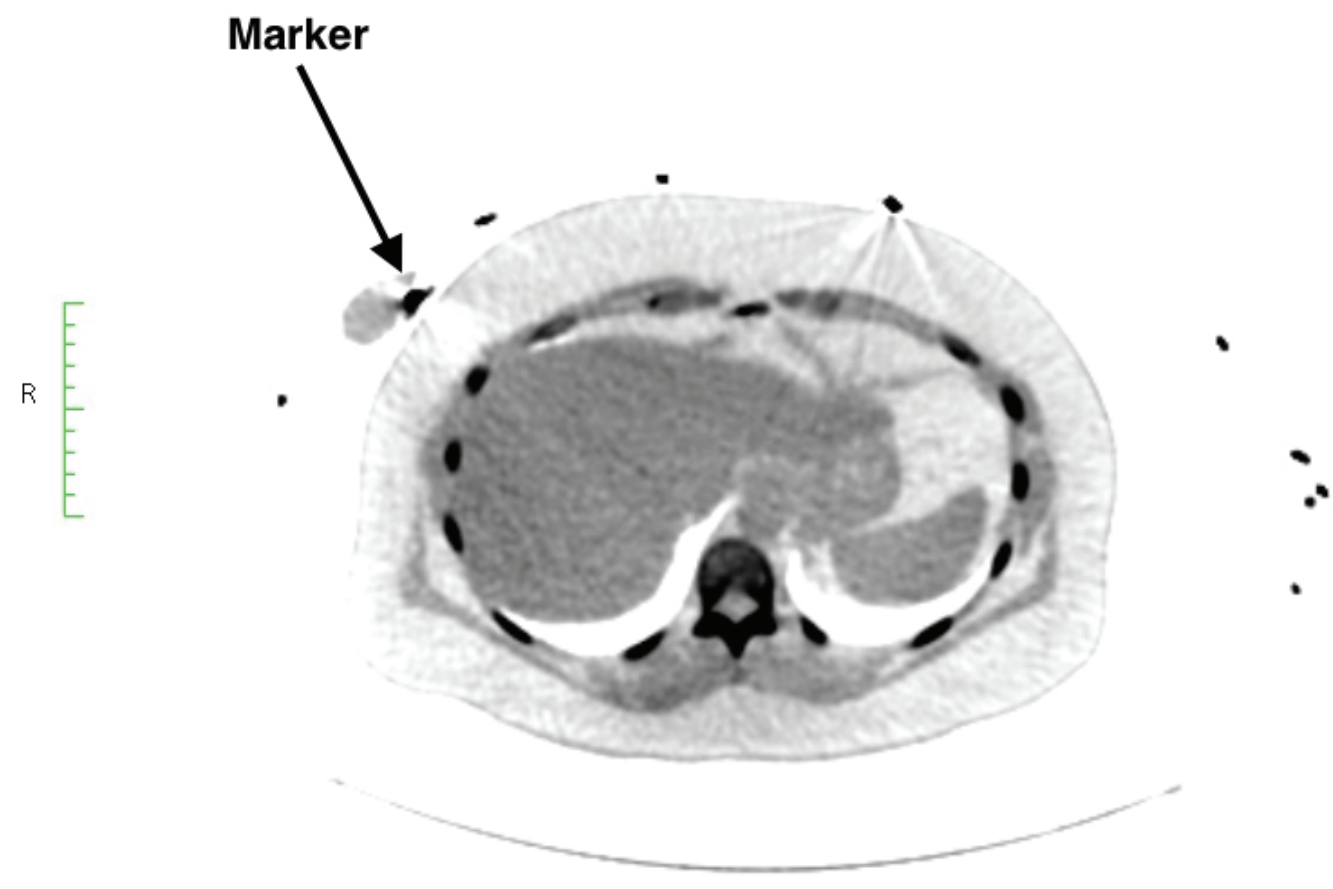

Zoom: 162\% Angle: 0 Im: $38 / 47$ I (S $\rightarrow$ ) $)$ Uncompressed

Figure 4.2: A CT-visible PeTrack fiducial marker is placed on a patient's abdomen. Its location on the attenuation correction CT scan can be used to initialize the tracking algorithm. 


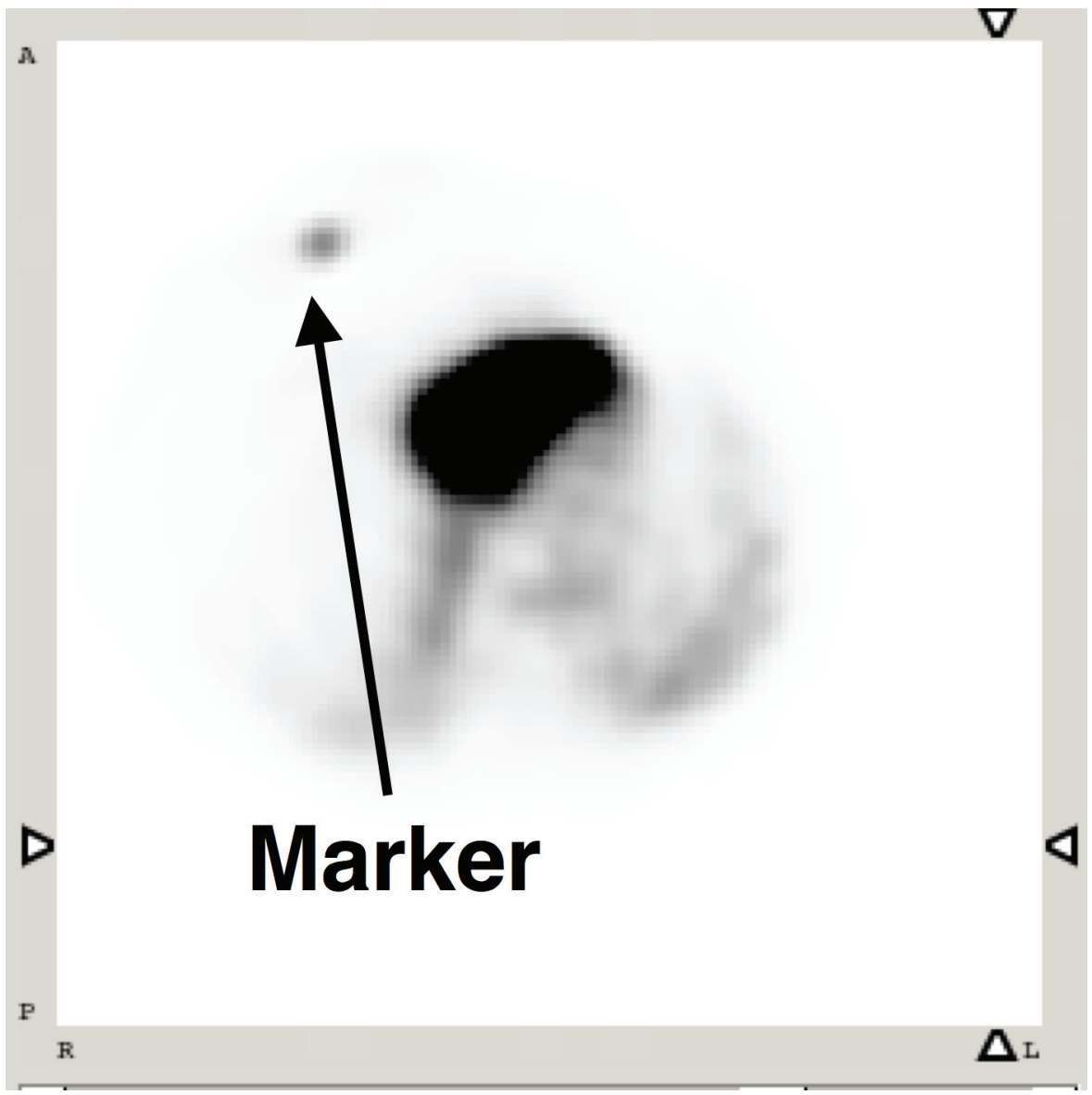

Figure 4.3: A PeTrack fiducial marker is visible on a transverse slice of a dynamic PET series. This image is the first frame in a series of 3215 -s frames. The activity of the marker is approximately $92.5 \mathrm{kBq}$. 


\subsection{Background rejection}

Rejecting the background from the physiological tracer activity within the patient is a challenging task. Both the tracer and the PeTrack marker use radioactive isotopes that decay by positron annihilation and produce back-to-back $511 \mathrm{keV}$ gamma rays. The background cannot be distinguished from the marker's signal by energy discrimination.

On the other hand, the spatial distribution of the background events differs from the marker's events. Geometric and physical arguments can be used to reject a very large number of background events. The following changes were made to the algorithm.

\subsubsection{Rejection by ROI and TOF}

First, a spherical ROI with radius $30 \mathrm{~mm}$ is defined around the estimated marker's location. This choice assumes that the real location of the marker is within $30 \mathrm{~mm}$

of the estimated location. Any coincidence event that does not intersect this ROI is discarded by the algorithm. The relative size of the ROI compared to positron range ensures that most real coincidence events from the marker are kept. The size of the ROI also allows for motion of the marker between localizations by the algorithm.

Second, time of flight (TOF) information is used to discard further events, if available on the PET scanner. As described in Section 2.1.6, the location of the positron annihilation along a LOR can be determined using Equation 2.5. A scanner with timing resolution of $550 \mathrm{ps}$ has a positional resolution of approximately $8 \mathrm{~cm}$. For the the first run of the algorithm, events are discarded by the algorithm if the calculated location of their annihilation is more than $8 \mathrm{~cm}$ away from the initialization location. For subsequent runs, events more than $8 \mathrm{~cm}$ from the current location of the marker are discarded. 


\subsubsection{Determining the optimal ROI size}

Following the two rejection methods described above, the background is still very large compared to the marker's signal. Thus the ROI should be made smaller. However, if the ROI is made too small, the real location of the marker might fall outside the ROI. In this situation several real coincidence events from the marker would be rejected. The ROI needs to be made small enough to reject enough background events, but large enough to encompass the real location of the marker.

The optimal size of the ROI can be determined from the spatial distribution of the events. I ran Monte Carlo simulations to study this spatial distribution. A cylindrical water phantom (30 cm in diameter and $15 \mathrm{~cm}$ in length) filled with a uniform ${ }^{18} \mathrm{~F}$ background activity was simulated. The perpendicular distance between the position of a fiducial marker (represented with a point in $3 \mathrm{D}$ space) and each LOR from the background activity (represented as 3D lines) was calculated. This distribution is shown in Figure 4.4. The distribution of the perpendicular distance between the marker and the LORs from the background increases linearly with the distance to the marker.

On the other hand, in a homogeneous medium the probability that a positron emitted from the origin annihilates at a distance $r$ away is represented by a 3D PDF $f(r)$. It has been shown that this PDF can be modelled as a 3D Gaussian distribution centered on the origin. $[102,103]$ This model was shown by Palmer et al. $[102,103]$ to be in close agreement with experimental measurements by Derenzo. [104] According to this model, the distribution of annihilation locations around a point source in a homogeneous medium is isotropic. Thus the 1D PDF $F(r)$ for annihilation at distance $r$ from the origin can be obtained by using spherical coordinates $(r, \phi, \theta)$ and integrating $f(r)$ over $\phi$ and $\theta$. This simple integration yields: 


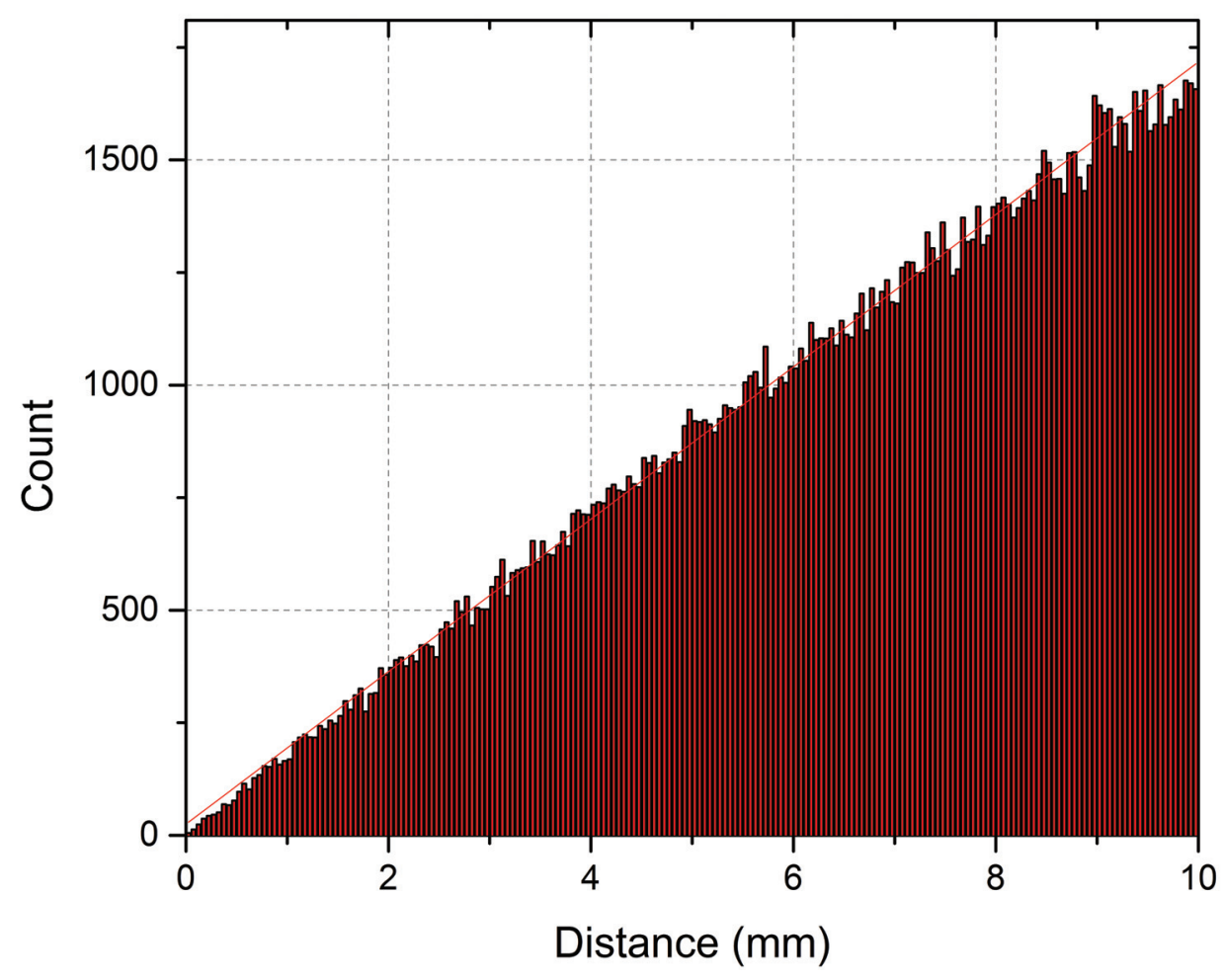

Figure 4.4: The distribution (0.05 $\mathrm{mm}$ bin width) of the perpendicular distance between a marker and the LORs from the background (90 $\mu \mathrm{Ci}$ activity).

$$
F(r)=4 \pi r^{2} f(r)
$$

This model explains the shape of the distribution shown in Figure 4.5, which shows the distribution of the distances between the marker and all LORs. The distinctive peak of the distribution is caused by the underlying 1D PDF $F(r)$ (Equation 4.1), which is not centered at the origin.

Consider Figure 4.6, which illustrates the method to determine the optimal size of the ROI. The data presented come from the same sample PET data mentioned in Section 4.2. The solid histogram corresponds to the distribution of the distance from 


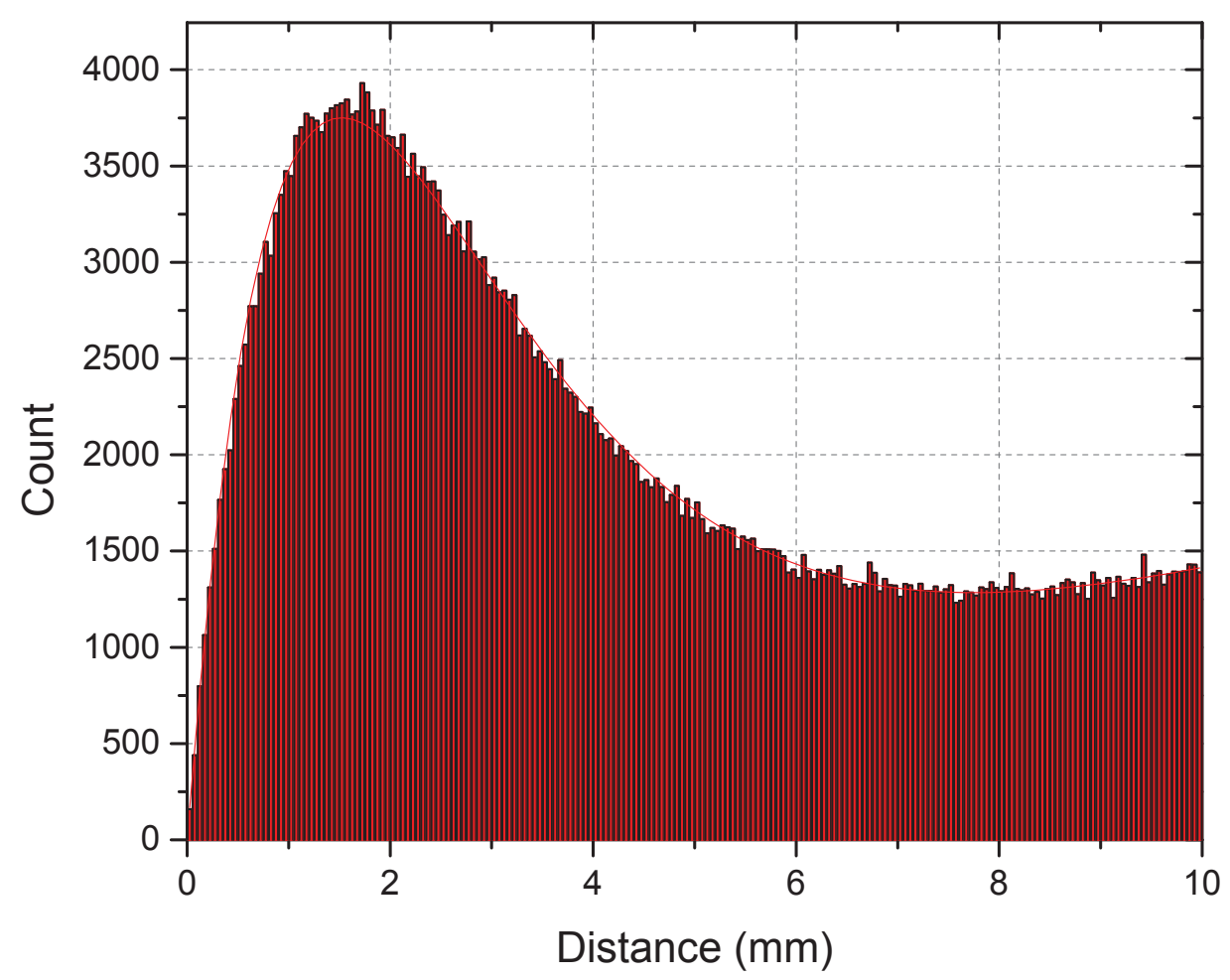

Figure 4.5: Distribution of the perpendicular distance (0.05 $\mathrm{mm}$ bin width) between a marker and all LORs. Events under the peak are expected to come from the marker. Events in the tail are a combination of events from the marker $(40 \mu \mathrm{Ci}$ activity) and from the background (90 $\mu \mathrm{Ci}$ activity). 
the marker's estimated location to all the coincidence events within the 0.5 s interval between localizations. As predicted by Monte Carlo simulations, a distinctive peak is followed by a tail which increases linearly. A linear fit is performed on the tail. On Figure 4.6, the blue line is the linear fit. The solid portion of the line indicates the data over which the fit is performed. The value of the linear fit at the centre of each bin is then subtracted from the content of each bin. The result is shown as the dashed red histogram. The first bin beyond the peak with a value less than or equal to 0 is $8 \mathrm{~mm}$; thus the size of the ROI is changed from $30 \mathrm{~mm}$ to $8 \mathrm{~mm}$. 


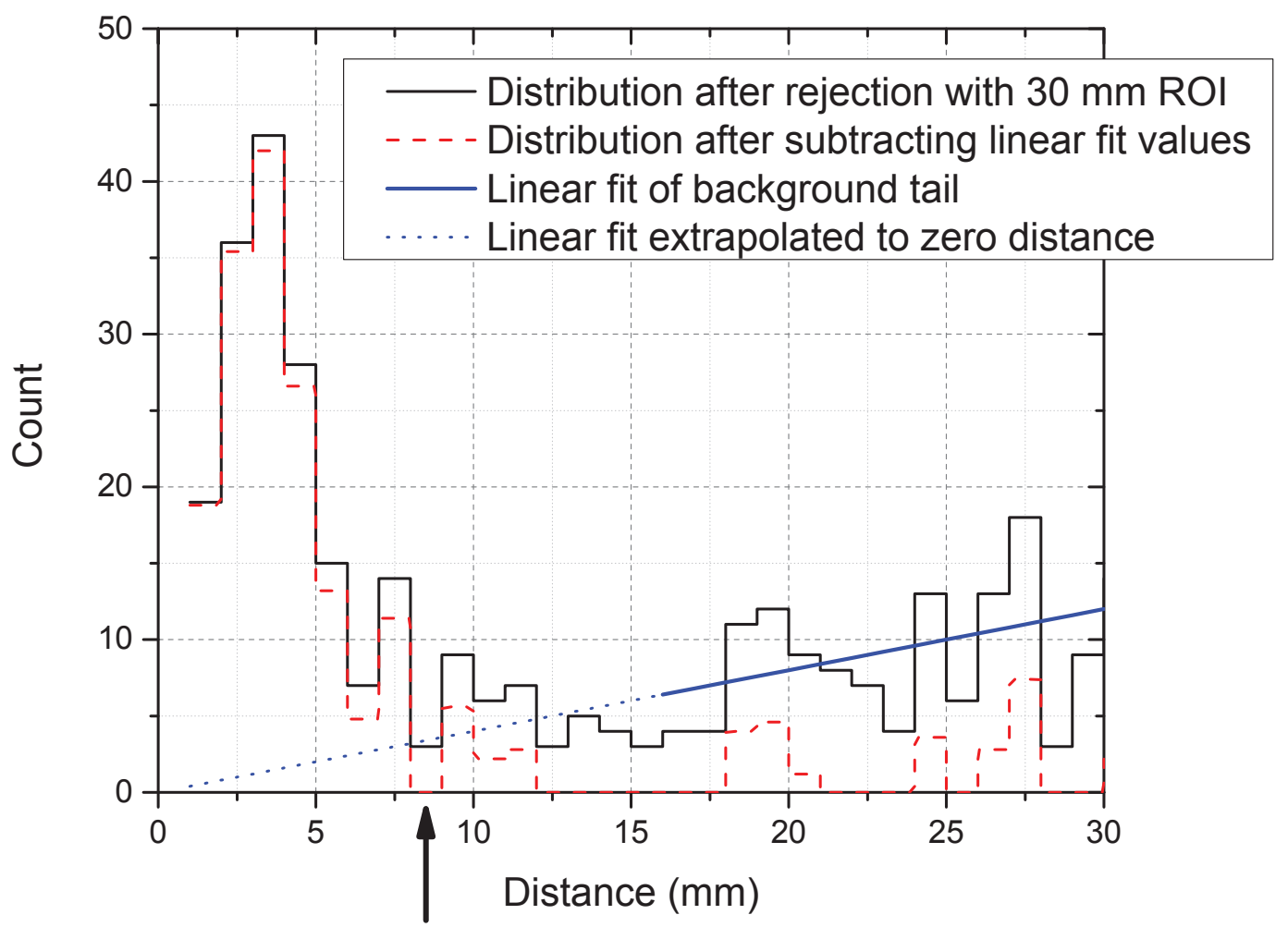

Figure 4.6: Illustration of the method to determine the size of the ROI for rejecting background events. The solid (black) histogram shows the distance between the estimated location of the marker and all coincidence events. A linear fit (solid blue line) is performed on the tail of this distribution and is extrapolated to zero distance (dashed blue line). The values of the fit at the centre of each bin are subtracted from the content of each bin. The dashed (red) histogram shows the value of each bin after subtraction of the background fit. The size of the $\mathrm{ROI}$ is set to the centre of the first bin beyond the peak with a value $\leq 0$; the arrow points to this bin on the figure. Note that this histogram is generated for every run of localization. 


\subsection{Flow of modified algorithm}

The following describes the flow of the modified algorithm through the first localization of the marker:

1. The algorithm is initialized manually using any of the methods outlined in Section 4.3.

2. Background events are rejected based on the ROI and TOF methods outlined in Section 4.4.1.

3. The size of the ROI is updated using the method described in Section 4.4.2.

4. Events that are beyond the updated ROI are discarded.

5. The EM algorithm proceeds as described in Section 2.2.2.

Every subsequent localization run of the algorithm repeats steps 2 through 5, using the previous location of the marker as its starting point. Figure 4.7 illustrates the background rejection methods in a simplified 2D manner.

It should be noted that this modified version of the algorithm can only track one marker at a time. However, multiple markers can be tracked independently: each marker can be tracked by a unique instance of the tracking program on a multi-core processor, as long as the markers are placed at least $10 \mathrm{~cm}$ away from each other.

Contrary to the use of PeTrack in radiotherapy (see Chapter 3), the current implementation of this algorithm cannot provide real time tracking during the PET scan. The total number of events detected in a typical cardiac PET scan is on the order of 250 million. Near the peak of the TAC curve, there might be more than $3 \times 10^{6}$ events to process every second. Thus, PeTrack should be used for retrospective motion tracking, i.e. it should process the list mode file after the scan has been acquired. 


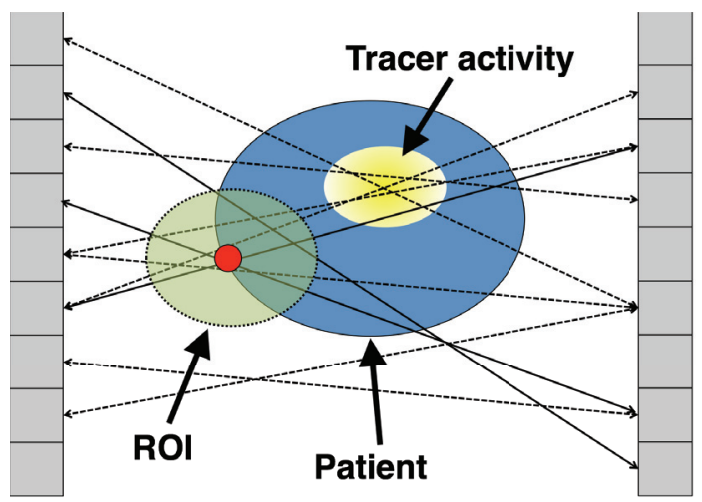

a)

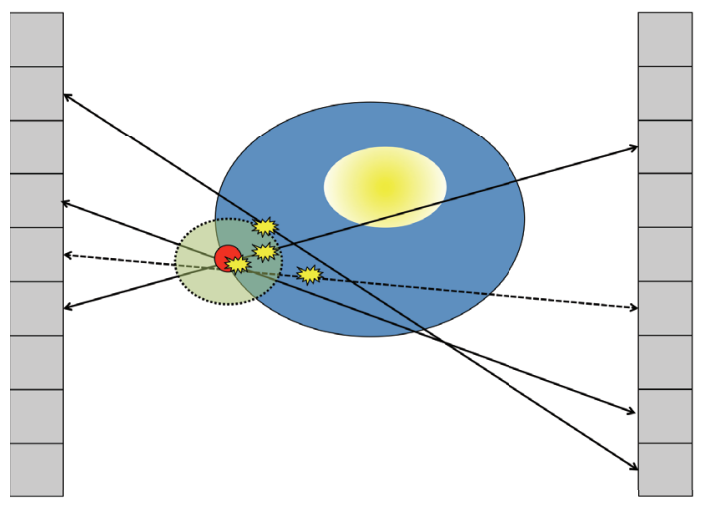

c)

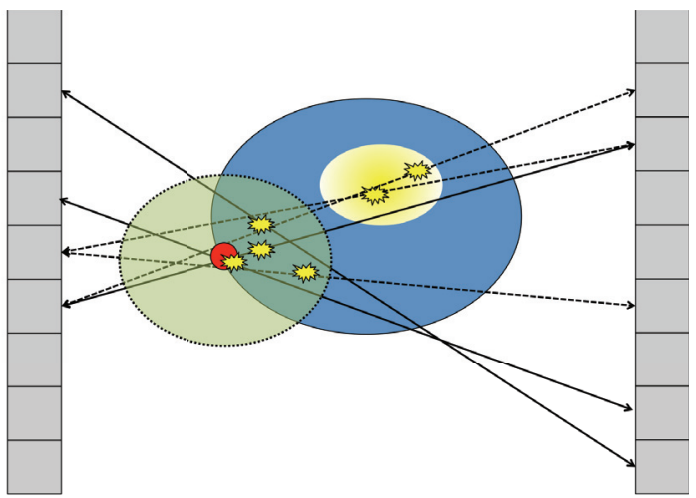

b)

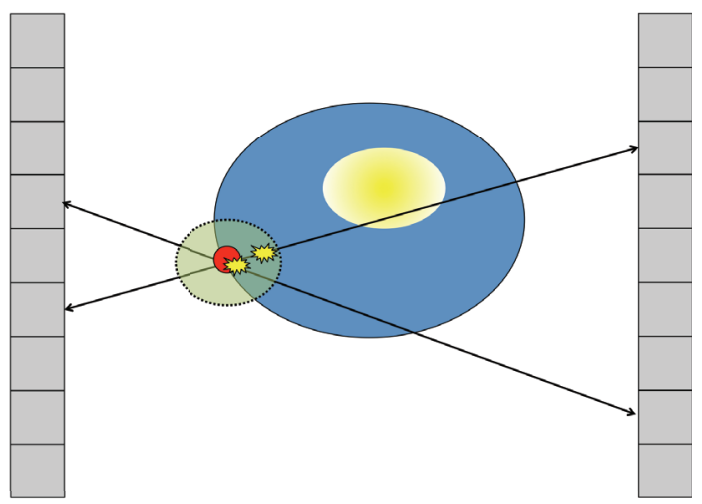

d)

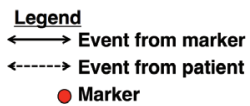

Annihilation location

Figure 4.7: Illustration of the background rejection technique. For simplicity, only two opposite rows of PET detectors are shown. a) ROI rejection: any event that is more than $30 \mathrm{~mm}$ away from the initial estimate of the marker's location is rejected. The ROI is represented by the dotted circle. b) TOF rejection: events whose estimated annihilation location is more than $80 \mathrm{~mm}$ from the marker are also rejected. c) Fitting for the optimal ROI size: the size of the ROI is adjusted by the fitting method. d) The ROI and TOF rejection methods are applied once more. 


\subsection{Evaluation using Monte Carlo simulations}

\subsubsection{Methods}

The accuracy and precision of PeTrack was evaluated with a mixture of Monte Carlo simulations and real clinical data. Although several dozen simulations were run with a variety of different parameters, for the sake of brevity one sample case is presented below.

Sample retrospective PET data from patients undergoing cardiac PET imaging were obtained from Dr. Rob deKemp from the University of Ottawa Heart Institute (UOHI). These data were acquired on a GE Discovery 690 PET/CT scanner (D690). The D690 is a full-ring PET scanner combined with a CT scanner. It uses LYSO as a scintillator, which has an average depth of interaction of $8.4 \mathrm{~mm}$ for $511 \mathrm{keV}$ photons. The detectors are arranged in 32 modules. Each module is made up of $2 \times 4$ (transaxial $\times$ axial) detector blocks which in turn are composed of $9 \times 6$ LYSO crystals. The ring diameter from block face to block face is $80.99 \mathrm{~cm}$. The transaxial FOV is $70 \mathrm{~cm}$ and the axial FOV is $15.67 \mathrm{~cm}$. Each crystal measures $4.18 \times 6.28 \times 25 \mathrm{~mm}^{3}$ (transaxial $\times$ axial $\times$ depth). The physical characteristics of the scanner are summarized in Table 4.1. The D690 uses a coincidence window of 4.9 ns. Bettinardi et al. [105] measured its average energy resolution at $511 \mathrm{keV}$ to be $12.4 \%$ and its timing resolution as $544.3 \mathrm{ps}$. The transaxial resolution of the scanner is $4.70 \mathrm{~mm}$ and $5.06 \mathrm{~mm}$ at $1 \mathrm{~cm}$ and $10 \mathrm{~cm}$ off axis, respectively; its axial resolution is $4.74 \mathrm{~mm}$ and $5.55 \mathrm{~mm}$ at $1 \mathrm{~cm}$ and $10 \mathrm{~cm}$ off axis, respectively. Its average sensitivity between 0 and $10 \mathrm{~cm}$ off axis is $7.5 \mathrm{cps} / \mathrm{kBq}$. The standard image reconstruction method on the D690 is an iterative 3D ordered subset expectation maximization (3D-OSEM) algorithm. All typical PET data corrections (scatter, random, dead time, normalization, and attenuation) are integrated in the iterative reconstruction. 
A spherical ${ }^{22} \mathrm{Na}$ marker with radius $0.25 \mathrm{~mm}$ and activity $92.5 \mathrm{kBq}(2.5 \mu \mathrm{Ci})$ was simulated with the GATE Monte Carlo package. [106] A cylindrical water phantom of radius $16 \mathrm{~cm}$ was included to provide scatter and attenuation of the marker. The marker was positioned at the surface of the cylinder to simulate a marker placed on a patient's abdomen. Clinically, the marker would be placed on the lower right side of the abdomen, away from the heart. This would ensure that the marker does not interfere with the reading of the image. The simulated marker moved sinusoidally in $3 \mathrm{D}$ according to the equation:

$$
\mathbf{r}(t)=\mathbf{r}_{0}+\mathbf{A} \sin \left(\frac{2 \pi t}{T}-\phi\right)
$$

where $\mathbf{r}_{0}$ is the initial offset in millimetres in each of the three anatomical directions (LR, AP, and IS; $\left.\mathbf{r}_{0}=(120,80,0)\right), \mathbf{A}$ is the amplitude of motion vector $(5 \mathrm{~mm}), T$ is the period of motion $(4 \mathrm{~s})$, and $\phi$ is a phase shift $(\pi / 2)$. The simulated scanner was modelled after the D690 scanner used clinically at the UOHI.

Using simulated time stamps, the simulated coincidence events from the marker were interlaced in the real patient list mode. This approach provided well-controlled marker motion with realistic patient background activity, while keeping the simulation time short. In addition, the events from the simulated marker were tagged in order to distinguish them from the events from the background activity.

\subsubsection{Conversion from list mode to Cartesian coordinates}

The current PeTrack implementation requires coincidence events to be stored as a

pair of points in Cartesian coordinates. The pair corresponds to the two endpoints of the LOR. However, the list mode format from the D690 stores coincidence events as a pair of two crystal indices, $\left(X_{1}, Z_{1}\right)$ and $\left(X_{2}, Z_{2}\right)$ (transaxial, axial). Each pair of indices uniquely identifies a detector element. Using the known geometry of the D690 
Table 4.1: Geometry of the D690. The gap is the distance between two consecutive blocks or two consecutive crystals.

\begin{tabular}{cc}
\hline \hline Parameter & Value \\
& $($ transaxial $\times$ axial $)$ \\
\hline Number of modules & 32 \\
Blocks per module & $2 \times 4$ \\
Crystals per block & $9 \times 6$ \\
Crystal size & $4.18 \times 6.28 \times 25 \mathrm{~mm}^{3}$ \\
Block size & $38.05 \times 38.05 \mathrm{~mm}^{2}$ \\
Block gap & $1.5 \times 1.5 \mathrm{~mm}^{2}$ \\
Crystal gap & $0.05375 \times 0.074 \mathrm{~mm}^{2}$ \\
FOV & $700 \times 156.7 \mathrm{~mm}^{2}$ \\
Ring diameter & $809.9 \mathrm{~mm}^{2}$ \\
Average depth of interaction & $8.4 \mathrm{~mm}$ \\
\hline \hline
\end{tabular}

(see Table 4.1), the location $\left(x_{d}, y_{d}, z_{d}\right)$ of the centre of any detector element (at the average depth of interaction) can be calculated in a straightforward manner. Thus, each event stored in the D690 list mode files was converted to a Cartesian coordinate format.

\subsubsection{Results}

The effectiveness of the background rejection techniques described in Section 4.4 was evaluated by quantifying the number of events from the marker used by the algorithm at various steps of the process (see Figure 4.8). When the TAC reaches its peak activity (approximately 30 to 45 s into the scan; see Figure 4.1), the marker's signal (including true, scattered, and random coincidences) accounts for less than $0.02 \%$ of the total events recorded within the localization time interval of $0.5 \mathrm{~s}$. After applying the 30-mm ROI and TOF rejection to those events (Step \#2 from Section 4.5), the 
fraction of events left originating from the marker increases by a factor of 100 to approximately $2 \%$. After optimizing the size of the ROI (Step \#3) and filtering the events with the ROI and TOF method again (Step \#4), the fraction of events from the marker increases by a factor 25 to about 50\%. Approximately $90 \mathrm{~s}$ into the 8minute scan, the background activity never contributes more than $25 \%$ of the total events used by the algorithm.

On average, 128 total events are used per localization after background rejection. In addition, $(41 \pm 4) \%$ of the total initial events from the marker are kept after background rejection. The total events from the marker initially include $35 \%$ of scattered coincidences; approximately $26 \%$ of the true coincidences from the marker are lost through the background rejection steps.

Figure 4.9 shows the location of the simulated marker as located by the PeTrack algorithm. The figure also includes the real location of the marker, as defined by Equation 4.2. The 3D localization error was also calculated. The error is defined as the 3D distance between the simulated location of the marker $\mathbf{r}(t)$ and the calculated location of the marker by the algorithm at time $t$. Figure 4.10 shows the distribution of the $3 \mathrm{D}$ error. The mean of the distribution is $(0.8 \pm 0.3) \mathrm{mm}( \pm 1 \mathrm{SD})$. 


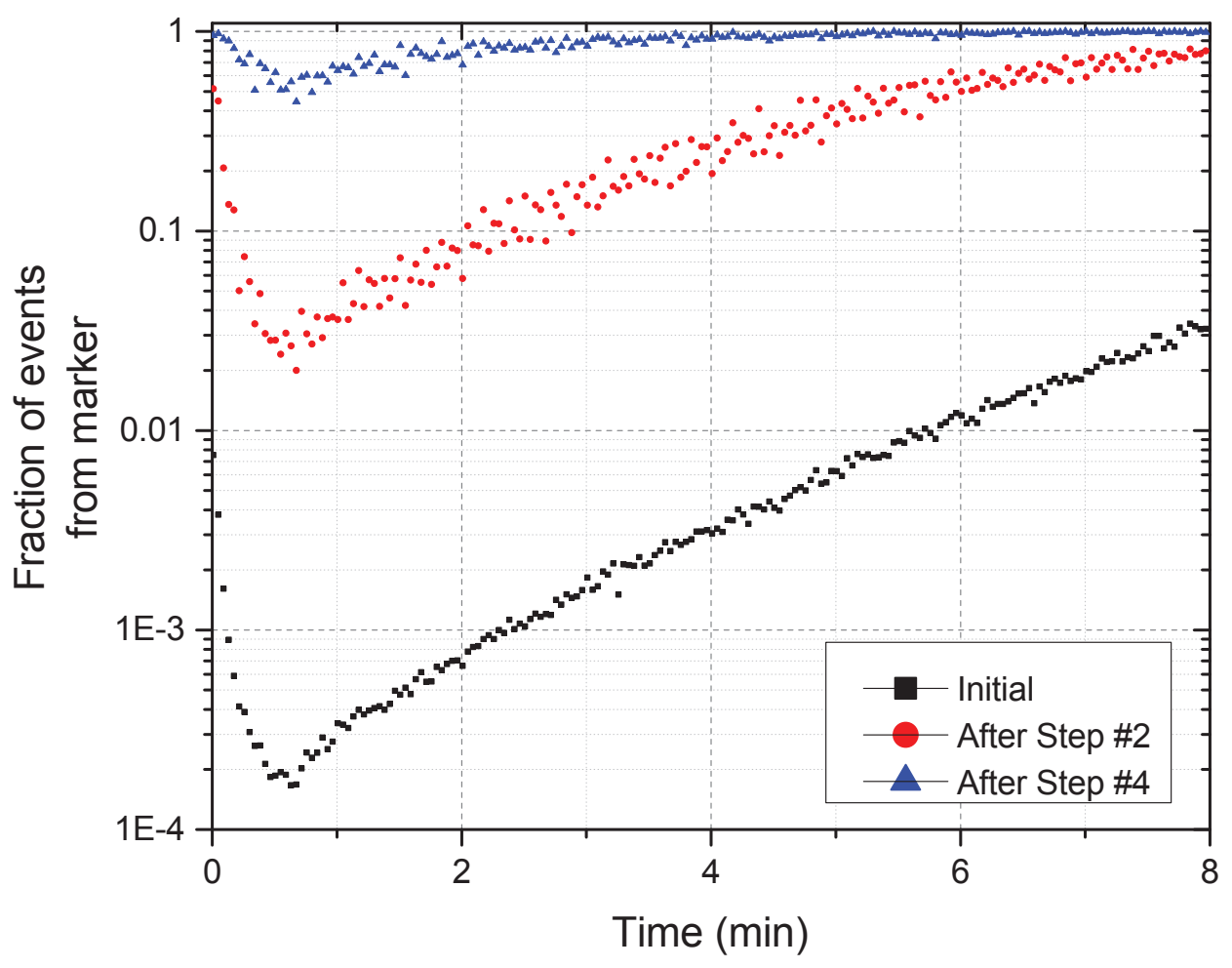

Figure 4.8: Fraction of the events used by the algorithm that originated from the marker. Initially (black squares), the vast majority of events are from the patient background activity. The fraction is increased by a factor 100 after rejecting events based on a $30-\mathrm{mm}$ radius ROI and TOF data (red circles). Near the peak of the TAC, another increase by a factor 25 is achieved by optimizing the size of the ROI through linear fitting. Note: every sixth data point is shown for visual clarity purposes. 


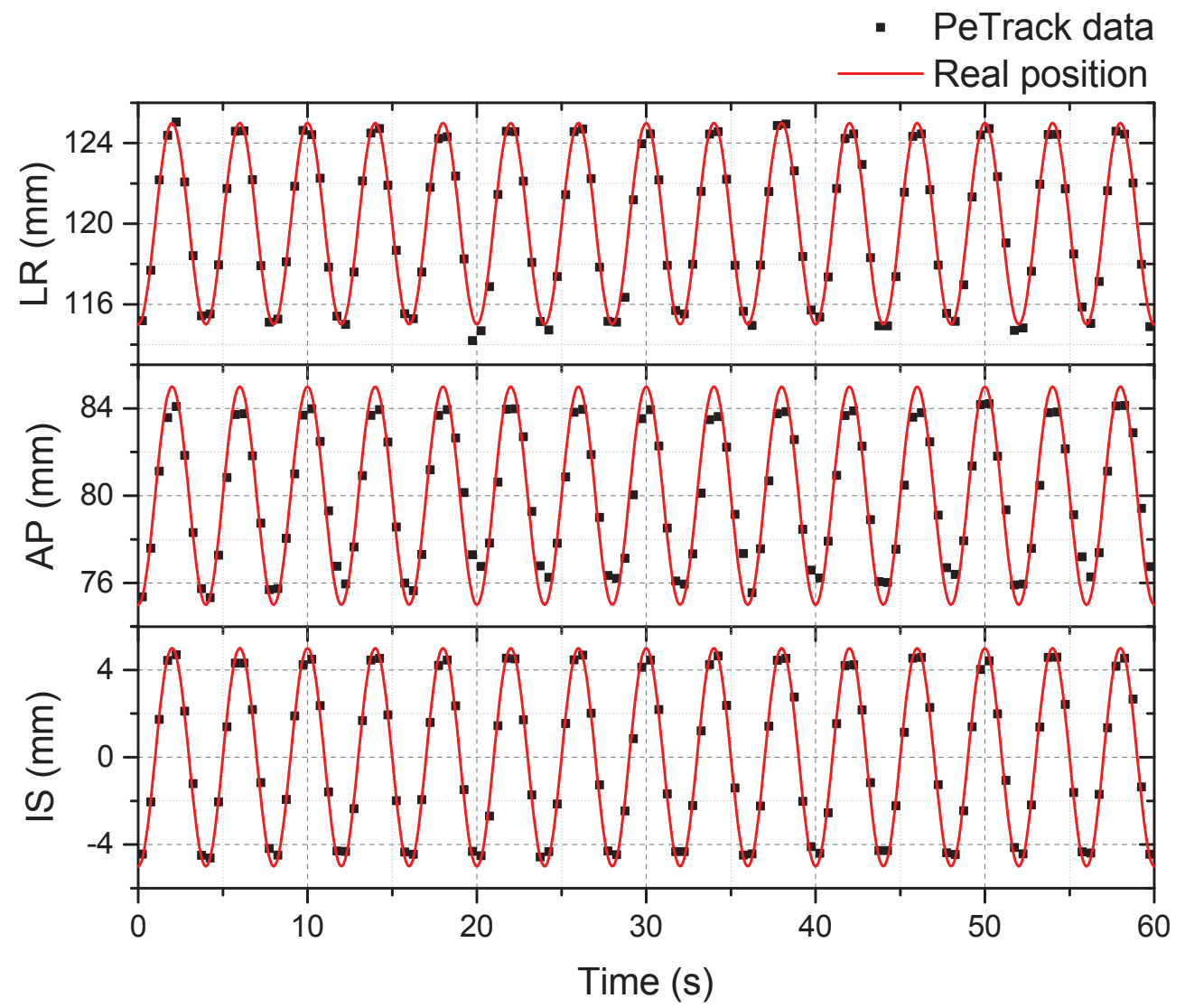

Figure 4.9: Location of the simulated marker (data points) along the three anatomical directions, as calculated by the PeTrack algorithm. The real location of the marker is shown by the solid red line and is defined by Equation 4.2. 


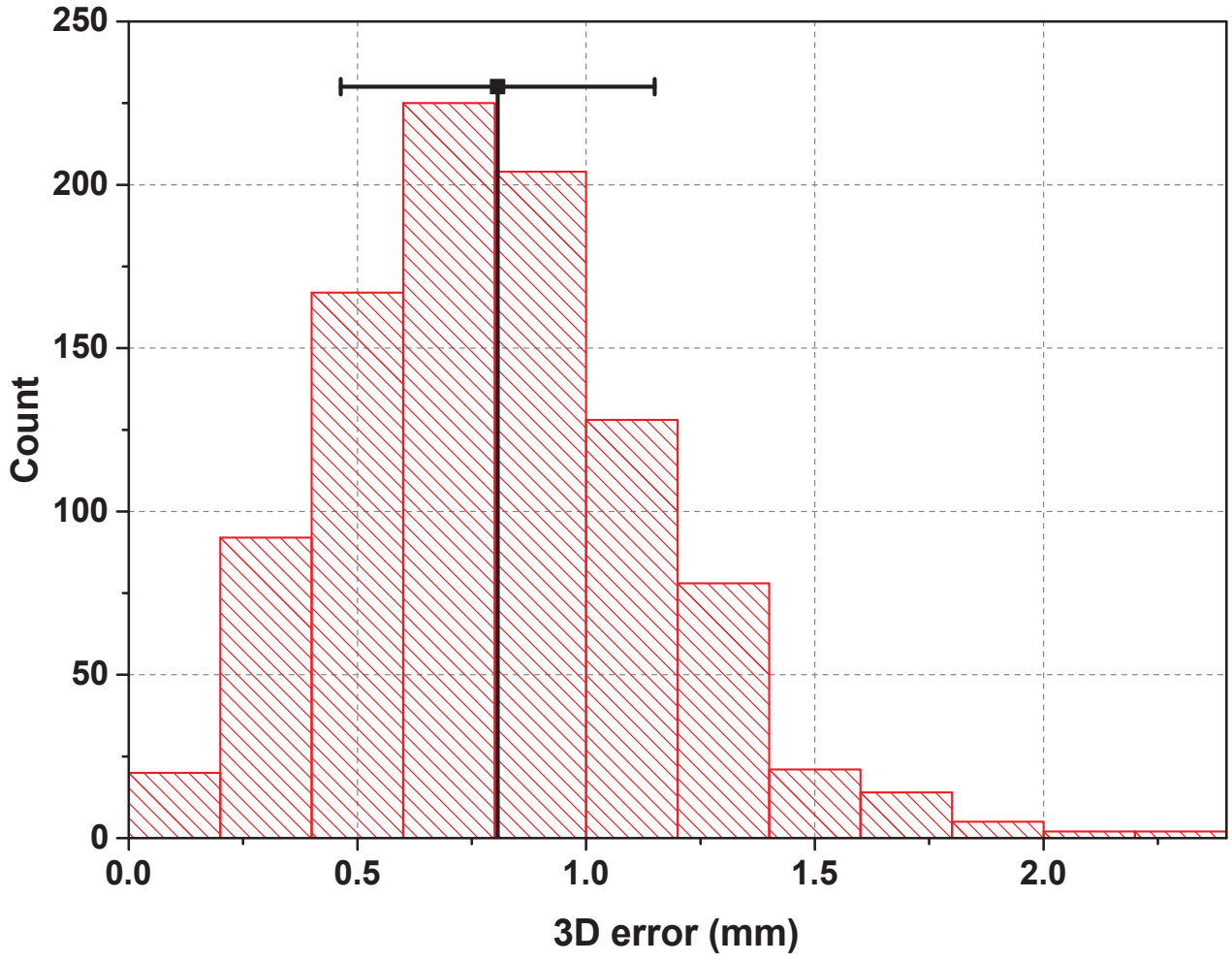

Figure 4.10: Distribution of the 3D error. The 3D error is the distance between the location of the marker $\mathbf{r}(t)$ and the location calculated by PeTrack at time $t$. The average of the distribution is $(0.8 \pm 0.3) \mathrm{mm}( \pm 1 \mathrm{SD})$. 


\subsection{Experimental evaluation with a dynamic phantom}

\subsubsection{Methods}

An experimental phantom study to evaluate the performance of PeTrack during PET imaging was conducted at the UOHI.

Three ${ }^{22} \mathrm{Na}$ fiducial markers were placed on an anthropomorphic torso phantom (Figure 4.11). The markers used $^{1}$ are made of an acrylic capsule $2.54 \mathrm{~cm} \times 6.35 \mathrm{~mm}$ (depth $\times$ height). The active core is a sphere with a diameter of $1 \mathrm{~mm}$. A 2-mm ring of bone equivalent material surrounds the active core. The activity of the three markers on the day of the experiment ranged from $81.4 \mathrm{kBq}(2.2 \mu \mathrm{Ci})$ to $150 \mathrm{kBq}$ $(4.1 \mu \mathrm{Ci})$. The markers were fixed to the surface of the phantom using modelling clay. The clay also served a second purpose. Radioactivity from markers outside the body of the patient can cause the scanner's reconstruction algorithm to overestimate the scatter. [107] The markers should appear to be within the outline of the patient body generated from a CT scan. This can be accomplished by placing sufficient water-equivalent material around the marker. Büther et al. suggest to use at least $10 \mathrm{~mm}$ of water-equivalent material around external markers; this is enough for the reconstruction algorithm to treat them as if they were within the patient body. [100]

The phantom used ${ }^{2}$ simulates the upper torso of a patient. It includes various inserts such as lungs, liver, spine, and heart. The main cavity of the phantom was filled with water. The phantom was placed on a motor-driven motion platform (see Figure 4.11). The phantom was scanned three times with a GE Discovery 690 PET/CT scanner. Each scan lasted 8 minutes. Before each scan, CT images were acquired and

\footnotetext{
${ }^{1}$ Model MMS03, Eckert \& Ziegler Isotope Products, Valencia, CA

${ }^{2}$ Model ECT/TOR/P, Data Spectrum Corporation, Hillsborough, NC
} 


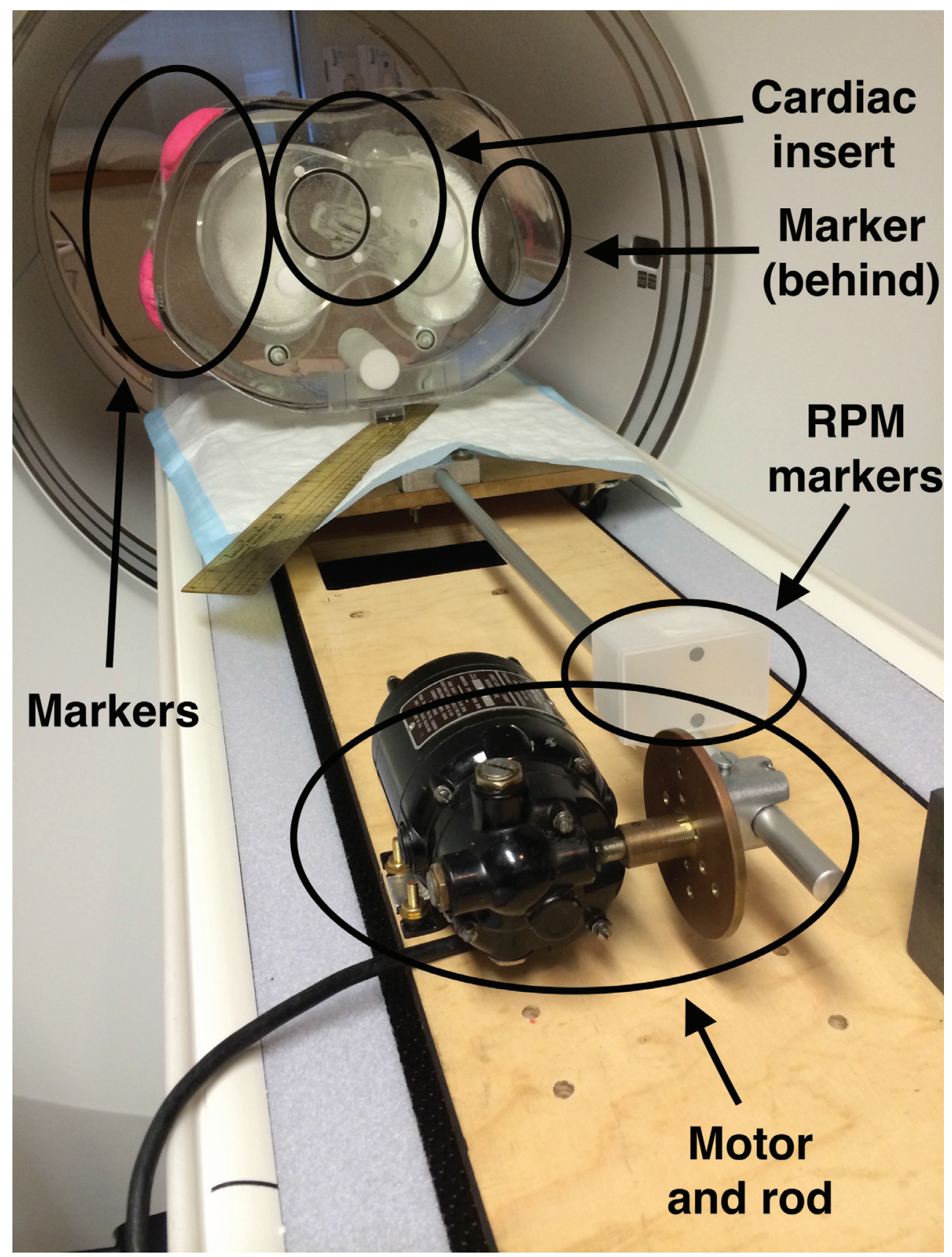

Figure 4.11: Anthropomorphic torso phantom used in the study. Three external fiducial markers are held onto the surface of the phantom with modelling clay. The main cavity of the phantom was filled with water and the cardiac insert was filled with a ${ }^{82} \mathrm{Rb}$ solution. The phantom was placed on a motor-driven platform. The block with reflective markers used by the Varian RPM system was attached to the rod. 
the cardiac insert was filled with a ${ }^{82} \mathrm{Rb}$ solution. The initial activity of ${ }^{82} \mathrm{Rb}$ in the heart insert was approximately $680 \mathrm{MBq}(18.4 \mathrm{mCi})$.

During the first scan, the phantom was static. During the second, the phantom was moved slowly in a periodic fashion in the IS direction. During the third, the period of motion was faster at $7.5 \mathrm{~s}$ to simulate breathing. In addition, a slow shifting of the phantom and a sudden movement near the end of the scan were added to simulate various patient body motions.

\subsubsection{Results}

The motion of each marker was tracked individually every $0.5 \mathrm{~s}$ throughout the 8minute scan ( $N=960$ localizations). Figure 4.12 shows the motion trace in the IS direction of one sample marker for all three scans. The phantom was kept motionless throughout scan \#1, so a flat trace is expected and observed. The small fluctuations represent the precision of the algorithm, i.e. its capacity to reproduce the same results under constant conditions. Motion of the phantom during the other two scans was driven by a rod attached to a vertical rotating plate; thus the motion traces are expected to be sinusoidal. In practice, scan \#2 is notably asymmetric on Figure 4.12. As can be inferred from Figure 4.11, the mostly rigid rod causes a small motion of the phantom in the vertical (AP) direction in addition to motion along the IS direction. However, the phantom was filled with water and weighed more than $24 \mathrm{~kg}$; the small motor driving the motion visibly struggled to move the phantom up during one half of the cycle. This caused the sinusoidal motion to resemble real breathing motion, which is known to spend more time in the end exhale phase than in the end inhale one. [108] The trace of scan \#3 on Figure 4.12 also demonstrates that PeTrack can track drifting motion and large sudden movements. In addition, this motion had slightly variable amplitude. This motion was caused by the rod gradually sliding out of the clamp holding it to the rotating plate of the motor. The discontinuous 


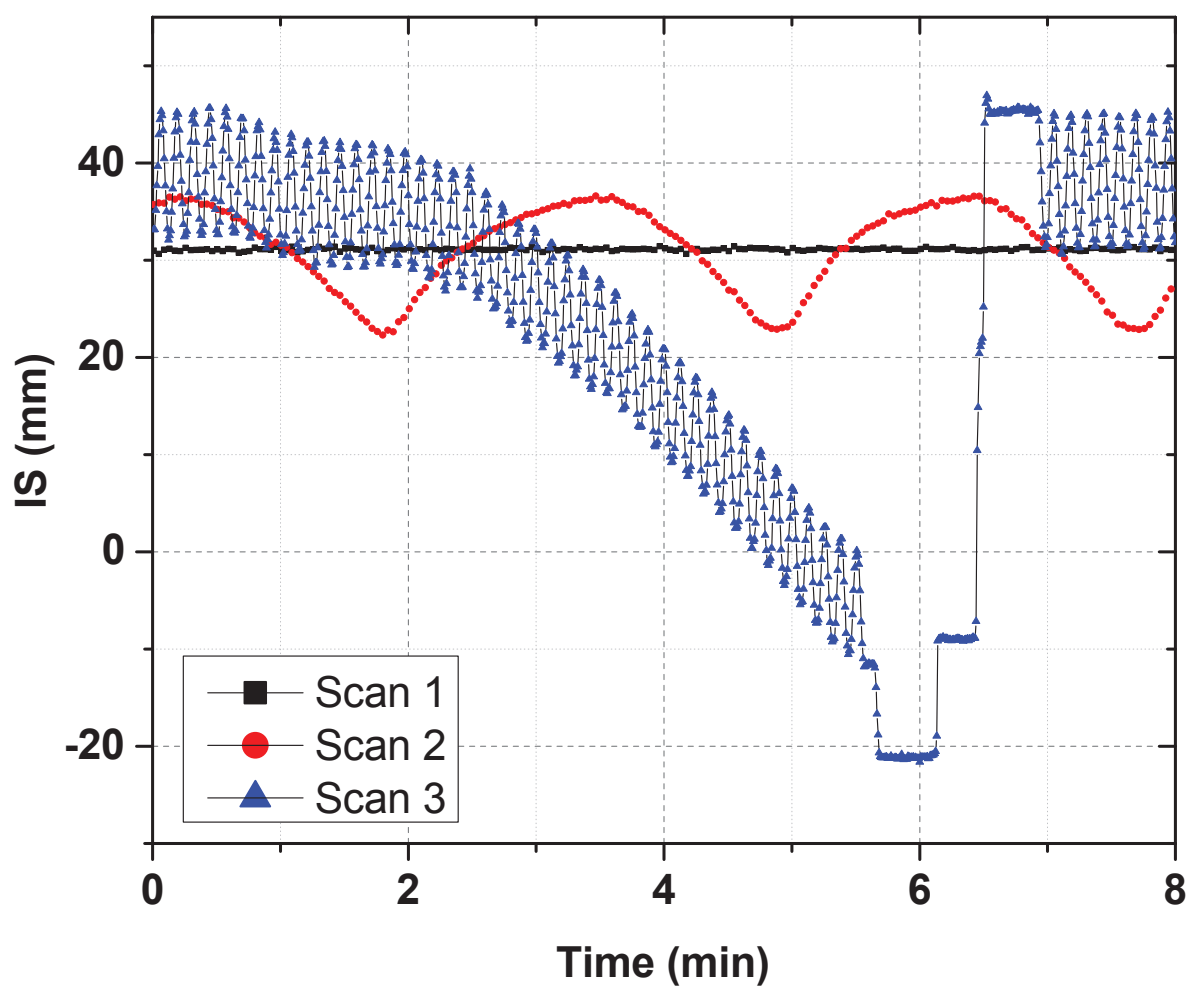

Figure 4.12: Motion traces of a sample marker for the static (\#1), slow (\#2), and fast and shifting (\#3) scans. 
movement around 6 minutes was caused by the rod falling out of the clamp and the phantom partly falling out of the FOV of the scanner. We re-attached the rod to the motor and replaced the phantom within the FOV before the end of the scan, without interrupting the data acquisition. This motion trace shows that PeTrack can track drifting variable-amplitude motion as well as large unpredictable motion. No user intervention besides replacing the phantom within the FOV was required.

The precision (or repeatability) of the algorithm is $0.14 \mathrm{~mm}$. This value was estimated by the following method:

1. The average location of each marker during the static scan was calculated.

2. For each of the 960 localizations of a marker, the 3D distance between the calculated location of the marker and its average location throughout the scan was calculated.

3. The standard deviation of the distribution of the 3D distance gives an estimate of the precision of the algorithm. This distribution is shown on Figure 4.13.

The real position of the markers within the coordinate system of the scanner is difficult to know accurately. In addition, the motion of the phantom is not easily modelled. Thus the relative (instead of absolute) accuracy was calculated. The relative accuracy is defined as the difference between the real distance between a pair of markers and the distance as calculated from the location of the markers provided by the tracking. Thus a negative relative accuracy means that the real distance between the markers is smaller than the distance as calculated from the location of the markers determined by PeTrack. The distance between each pair of markers was

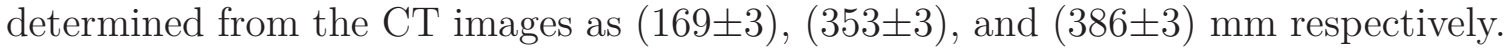
The uncertainty on the distance was estimated from the voxel size of the CT images: $(1.37 \times 1.37 \times 3.27) \mathrm{mm}^{3}$. The uncertainty on the $3 \mathrm{D}$ location of one marker 


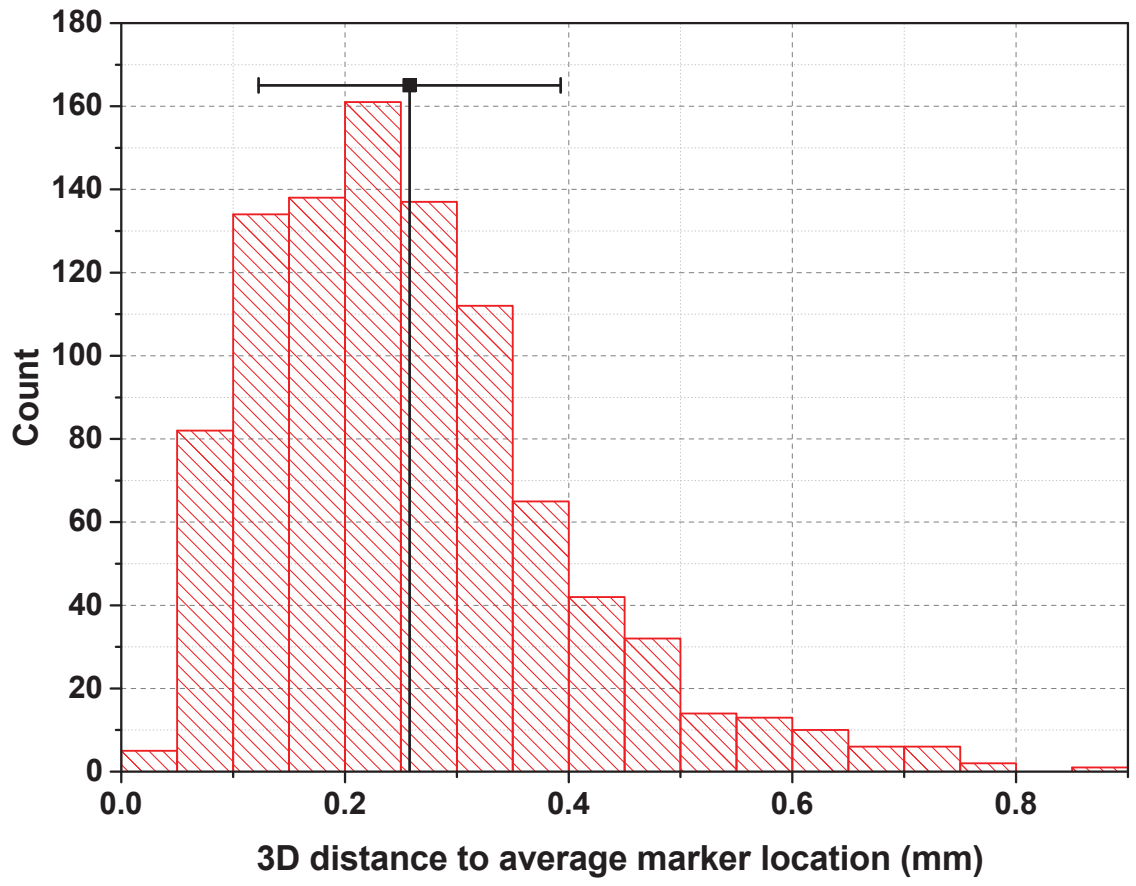

Figure 4.13: Distribution of the 3D distance between the calculated location of the marker and its average location throughout the scan. The mean of the distribution is $(0.26 \pm 0.14) \mathrm{mm}$. The precision of the tracking can be taken as the standard deviation of this distribution, i.e. $0.14 \mathrm{~mm}$. 
is $\sqrt{(1.37 / 2)^{2}+(1.37 / 2)^{2}+(3.27 / 2)^{2}}=1.9 \mathrm{~mm}$. When measuring the distance between two markers, the uncertainty on the 3D location of each marker is added in quadrature: $\sqrt{(1.9)^{2}+(1.9)^{2}} \approx 3 \mathrm{~mm}$.

Figure 4.14 shows the distribution of the relative accuracy among all three scans. ${ }^{3}$ The mean of the distribution is $(0.3 \pm 0.6) \mathrm{mm}$.

\footnotetext{
${ }^{3}$ The late part of the third scan was excluded from this analysis because two of the markers fell out of the field of view of the scanner.
} 


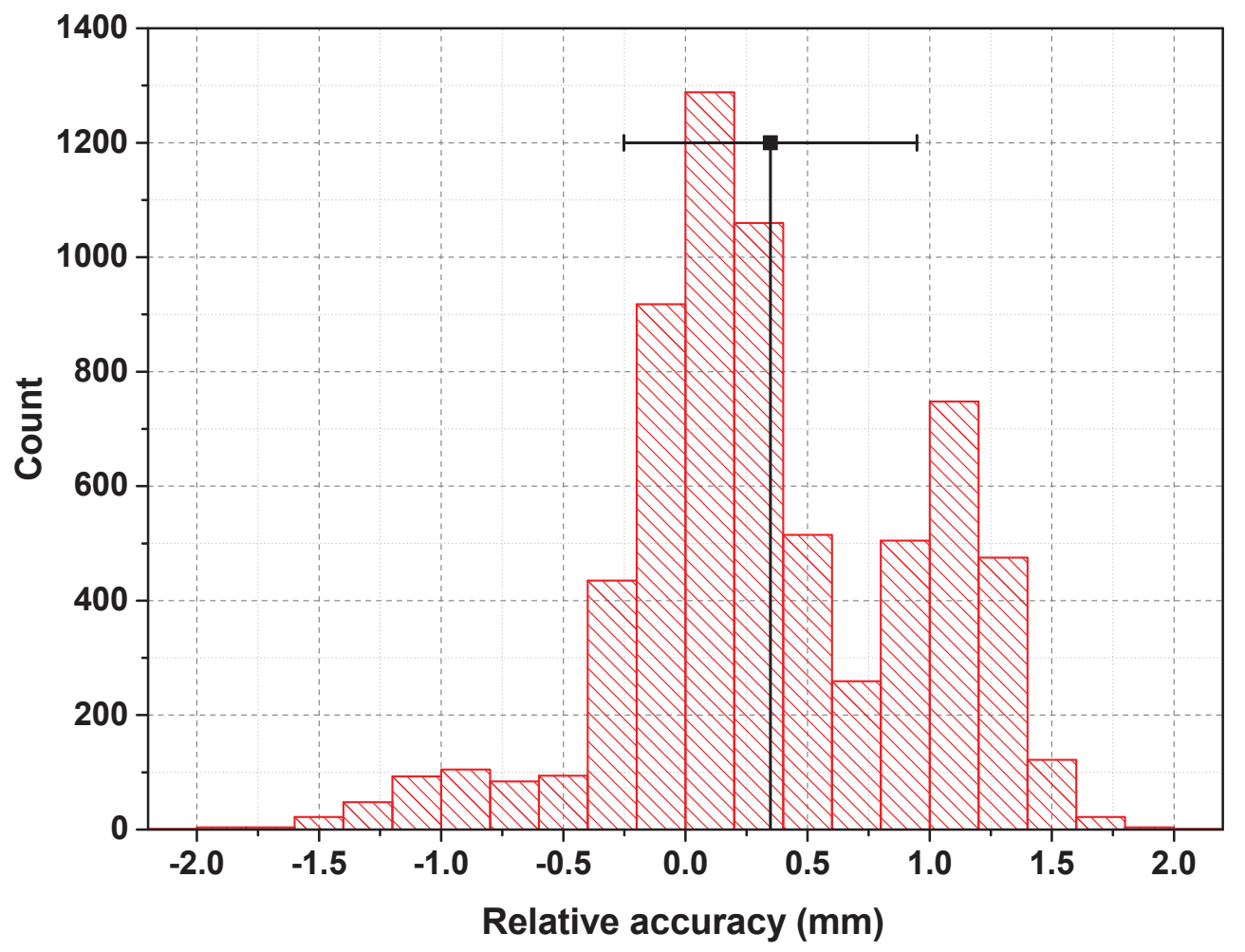

Figure 4.14: Distribution of the relative accuracy among all three scans. The relative accuracy is defined as the difference between the real distance between a pair of markers and the distance as calculated from the location of the markers determined by the algorithm. According to this definition, a negative relative accuracy indicates that the real distance between the markers is smaller than distance calculated from the location of the markers provided by PeTrack. The mean of the distribution is $(0.3 \pm 0.6) \mathrm{mm}$. 


\subsection{Discussion}

Results from the Monte Carlo simulations clearly show how effective the background rejection methods are at increasing the signal to noise ratio. This is crucial for motion tracking using PeTrack during PET imaging since otherwise, the signal from the marker is too weak to track accurately. Both the simulation and phantom studies confirm that the tracking can achieve submillimetre accuracy and precision. The results are in line with the performance of the tracking algorithm when no background is present (see Section 2.2.6). The phantom study confirms that the algorithm can track several types of motion concurrently, such as respiratory motion, baseline drift, and sudden patient motion. Further supporting evidence will be provided in Chapter 5. Locating the marker twice per second (sampling frequency of $2 \mathrm{~Hz}$ ) appears sufficient to resolve most types of motion. The sampling frequency can be decreased or increased, as long as sufficient events from the marker are detected within each sampling interval.

The performance of the tracking and successful tracking of a marker depend on a few key quantities: the initial estimate of the marker's location, the signal to noise ratio, and the number of events originating from the marker used for localization. Using the initialization method described above (see Section 4.3), the initial location of the marker can be estimated to within a few millimetres of its actual location. Several factors affect the other two quantities: the activity of the marker, the initial tracer activity injected in the patient, the sampling interval of the tracking, etc.

In particular, the position of the marker with respect to the edge of the axial FOV of the scanner has a larger effect on the number of events from the marker detected. As we move towards the edge of the axial FOV, the sensitivity decreases approximately linearly. [109] Quantitative data for the axial sensitivity of the D690 in the literature is scarce, but it can be estimated using Monte Carlo studies which 
use a non-validated model of the D690. After background rejection, an average of 227 events from the marker remain when the marker is placed in the centre of the axial FOV. When placed $33 \mathrm{~mm}$ away from the edge of the axial FOV, the number of events from the marker remaining after background rejection is 110. Assuming that this decrease is entirely due to the decreasing axial sensitivity of the scanner, then the marker has to be placed at a minimum of $18 \mathrm{~mm}$ away from the edge of the FOV to have at least 60 events remaining after background rejection. Xu et al. [25] have shown that the success rate of the algorithm rapidly decreases if fewer than 60 events is used per localization. The success rate falls from $99.7 \%$ to $70-80 \%$ at fewer than 30 events, if the error on the initial estimate of the marker's location is 5-15 mm. As an alternative, the activity of the marker could be increased to $7.5 \mu \mathrm{Ci}$ to ensure having enough events near the edge of the FOV. The activity of the marker would still be negligible compared to the tracer activity, but the effect of the increased activity on the reconstructed PET images should be assessed. Of course, the likelihood of the marker moving out of the FOV increases the closer the marker gets to the edge of the FOV.

Tracking motion with PeTrack during PET imaging requires no additional hardware and very little computing power. Fewer than five minutes on a $2.66 \mathrm{GHz}$ Intel Core i7 processor are typically needed to process the data from a scan which can contain more than $250 \times 10^{6}$ events. The marker used is low activity and is expected to have negligible impact on the radiation burden of the patient and medical staff. The signal of the marker is recorded by the scanner and stored within the list mode data. This provides several advantages. First, no spatial coregistration is needed between PeTrack and the PET scanner. Second, the tracking information is intrinsically synchronized with the PET data. Third, motion information is available for retrospective studies as long as the list mode data from the scan are preserved. By default, the team at the UOHI only keeps the motion trace from their current external 
respiratory gating system (Varian RPM) for the most recent scan of the patient. The motion information for the RPM system is stored in a file separate from the list mode file. Thus the motion trace of the RPM marker (and consequently, useful information about the motion of the patient during the scan) is lost after the patient's subsequent scan.

\subsection{Conclusion}

This chapter presented the modifications made to the PeTrack algorithm to track the motion of an external fiducial marker during PET imaging. The background rejection methods introduced greatly increase the SNR. The modified algorithm performs comparably to the original algorithm in terms of accuracy and repeatability. 


\section{Chapter 5}

\section{Motion compensation in PET imaging using PeTrack}

\section{$5.1 \quad$ Introduction}

The need for motion compensated PET imaging was established in Section 1.2. Once motion is detected, the information is used to apply motion compensation to the PET data. This can be done in wide variety of manners.

As in radiotherapy, respiratory gating is widely used. The most common respiratory gating method in PET imaging is phase gating: a particular phase of the breathing cycle is picked as a reference (e.g. end exhale or end inhale). Whenever the patient's breathing reaches this phase of the cycle, the current time stamp is recorded in the raw PET list mode. This time stamp is referred to as a respiratory trigger. Each full breathing cycle is then divided into eight equal size temporal gates. Coincidence events are retrospectively binned into those eight gates. Each gate is then reconstructed separately (see Figure 5.1). Other gating schemes are also possible such as amplitude gating and variable-size temporal gates. [110,111] Several of the motion tracking methods described in Section 4.1 are used as the basis for respiratory gating. [93, 94, 97, 100] 


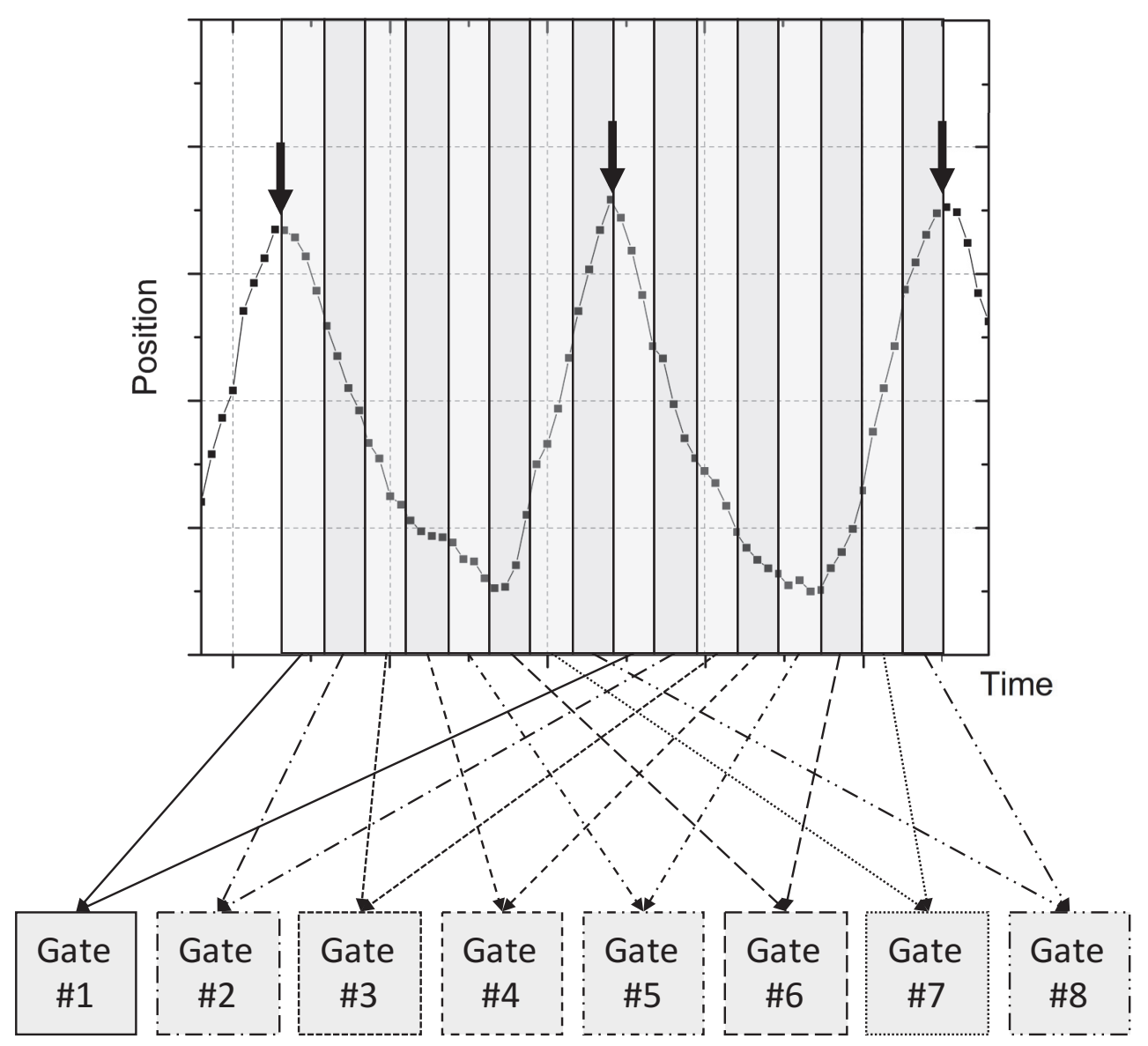

Figure 5.1: Illustration of the principle of phase gating. Respiratory triggers (arrows) correspond to the time of end inhale, in this example. The breathing cycle is divided into eight equal size temporal gates between two consecutive triggers. Events are retrospectively binned into those eight gates. 
The multiple acquisition frame (MAF) method reconstructs individual frames (gates) independently and aligns them to a reference frame. [112-116] The corrected frames may then be added together, if desired. The major drawback from this technique is that motion within individual frames is ignored. Furthermore, the SNR decreases with increasing number of frames, which leads to noisy images.

Another approach is to directly use the motion information in the image reconstruction process. [117-121] These methods modify the system matrix to account for motion. ${ }^{1}$ Some implementations of these techniques derive non-rigid motion information from image registration of multiple frames, a method similar to MAF. [122-124] Methods that modify the system matrix have the advantage of being able to incorporate non-rigid motion and to use all recorded events, including those events which fall outside the FOV after motion correction, but they are complex to implement.

Event-by-event (EBE) motion correction is also possible. The measured motion is used to apply a geometric transformation to the measured LORs and align them to a reference position. [123,125-127] EBE motion correction can correct for intraframe motion and can potentially be used in real time. [127] Since most implementations only correct for rigid body motion, it is widely used in brain imaging. [116, 128-131] EBE motion correction has been shown to produce better images than the MAF method. [116, 132]

\subsection{PeTrack for motion compensation in PET imaging}

The previous chapter demonstrated that PeTrack can track the 3D motion of a fiducial marker during PET imaging accurately and precisely. The motion trace is produced

\footnotetext{
${ }^{1}$ In PET image reconstruction, the system matrix is $\mathbf{P}=\left(p_{i j}\right)_{I \times J}$, where $p_{i j}$ is the probability that activity in voxel $j$ will be detected along LOR $i$.
} 
retrospectively, using only data from the raw PET data. The motion information can be extremely useful to produce motion compensated PET images.

In this chapter, two proof of concept applications of PeTrack towards motion compensated ${ }^{82} \mathrm{Rb}$ cardiac PET imaging are presented. First, the use of PeTrack for respiratory gated PET imaging was evaluated and compared to gated PET images using the Varian RPM system. Then the feasibility of motion correction at the list mode level for dynamic ${ }^{2}$ cardiac PET imaging was assessed on phantom and patient studies.

\subsection{Human heart}

The human heart is not exactly aligned along the three anatomical directions (LR, AP, and IS). Instead, three standard imaging planes are defined with respect to the anatomy of the heart: the short axis (SA), the horizontal long axis (HLA), and the vertical long axis (VLA). ${ }^{3}$ All three planes are shown in Figure 5.2. In cardiac PET imaging, we are particularly interested in the blood that the coronary arteries bring into the walls of the myocardium. It is customary to represent the perfusion of the walls of the heart with the aid of polar maps. Figure 5.3 shows the various coronary arteries and other anatomical landmarks of the heart and Figure 5.4 illustrates to what areas of a polar map they correspond.

\footnotetext{
${ }^{2}$ In dynamic imaging, the list mode data are binned into a number of consecutive time frames (e.g. 32 frames of duration $15 \mathrm{~s}$ each for an 8 -minute ${ }^{82} \mathrm{Rb}$ cardiac PET study). Each frame is reconstructed separately. The resulting images are referred to as a dynamic series or dynamic study.

${ }^{3}$ Note the unfortunately confusing use of the term "axis" to refer to a plane.
} 

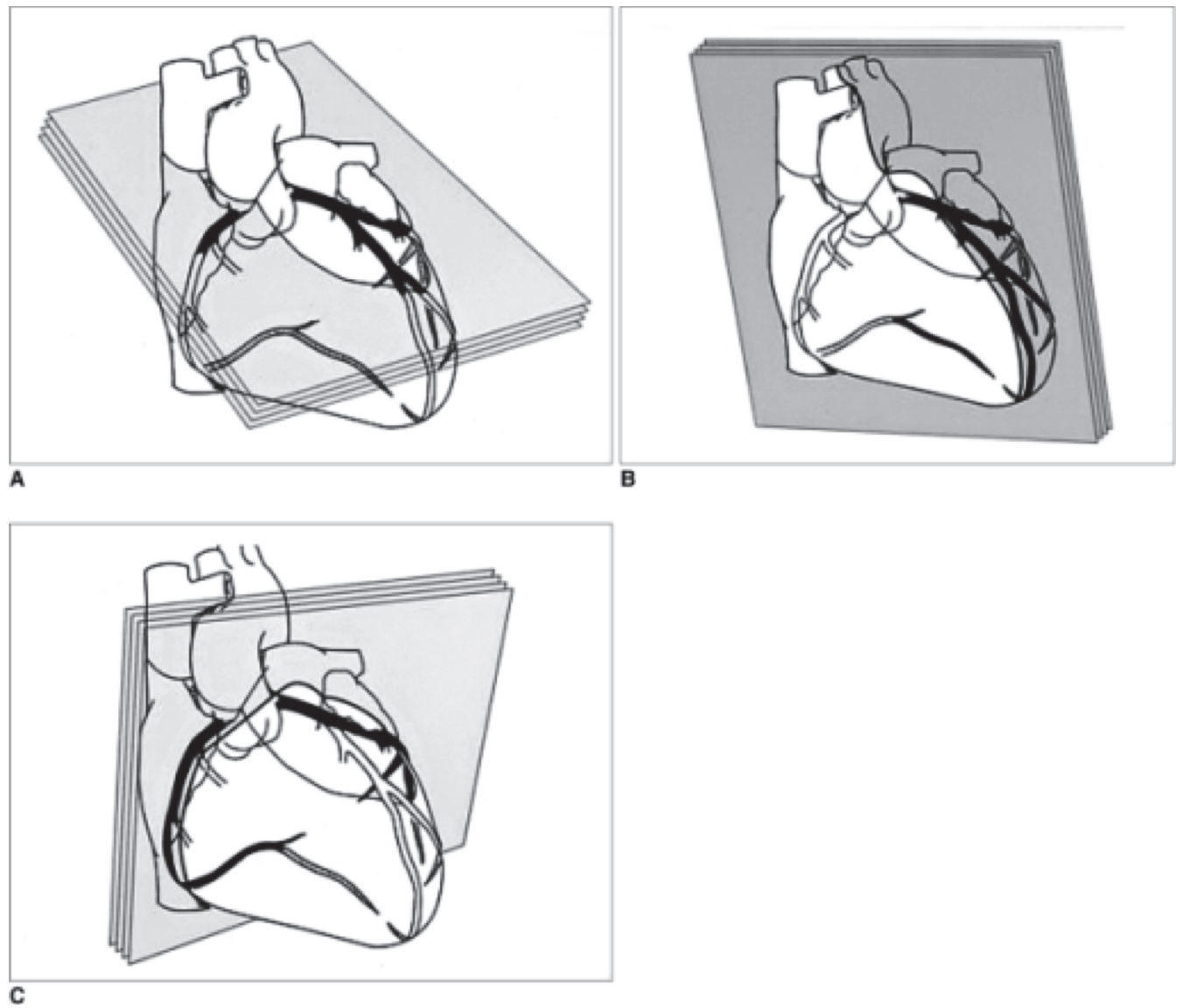

Figure 5.2: The three standard cardiac imaging planes of the heart: A) the horizontal long axis (HLA); B) the vertical long axis (VLA); and C) the short axis (SA). This figure is reproduced from Choi et al [4] with permission under a Creative Commons Attribution-NonCommercial 3.0 Unported License (CC BY-NC 3.0; http://creativecommons.org/licenses/by-nc/3.0/); copyright (C) 2006 The Korean Society of Radiology. 


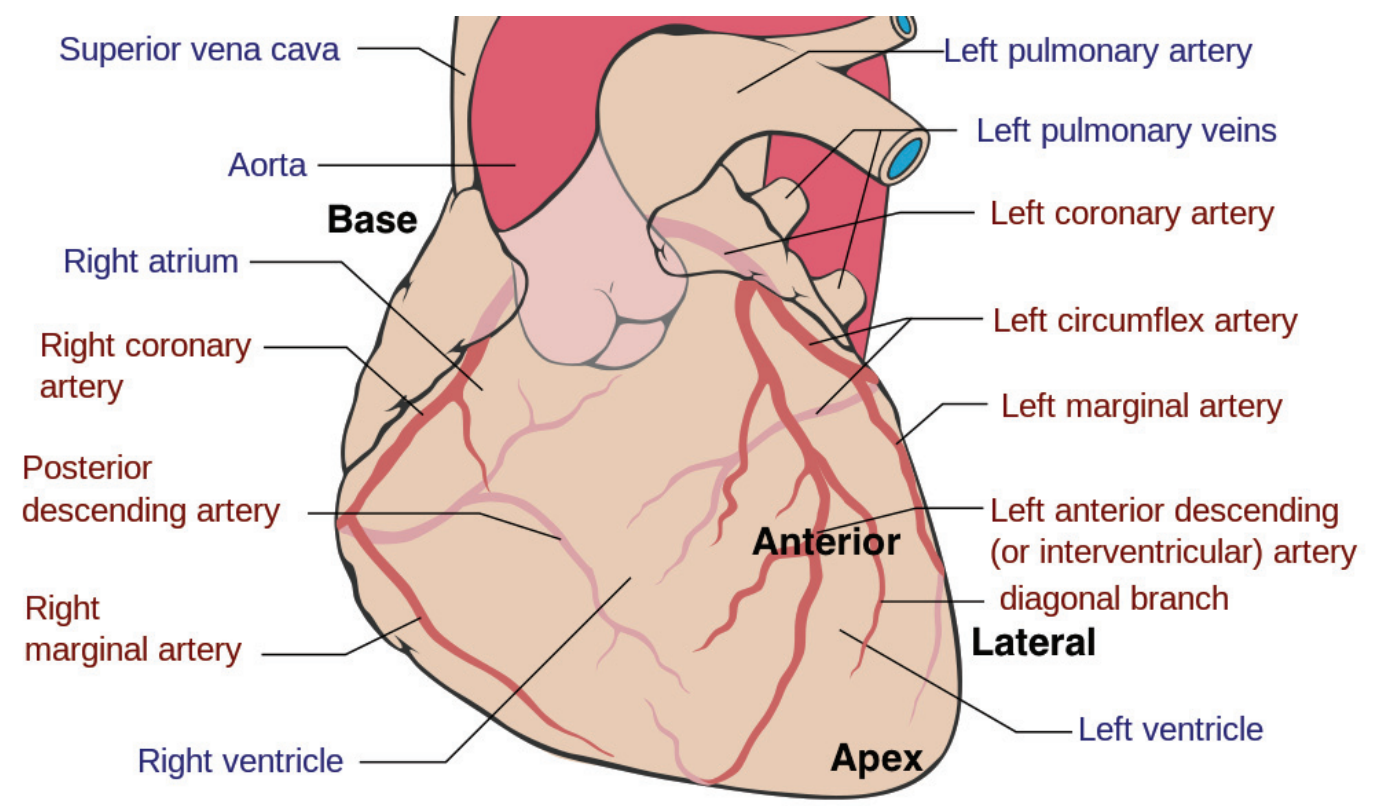

Figure 5.3: Anatomy of the human heart. Major coronary arteries are labeled in red; other landmarks are in blue or black. This figure is reproduced with permission under a Creative Commons Attribution-Share Alike 3.0 Unported License (CC BY-SA 3.0; http://creativecommons.org/licenses/by-sa/3.0/). Original figure Coronary.pdf by Patrick J. Lynch, medical illustrator; derivative work by Fred the Oyster; adaptation and further labeling by Mikael Häggström. Additional labels in black were added to this version by Marc Chamberland. 


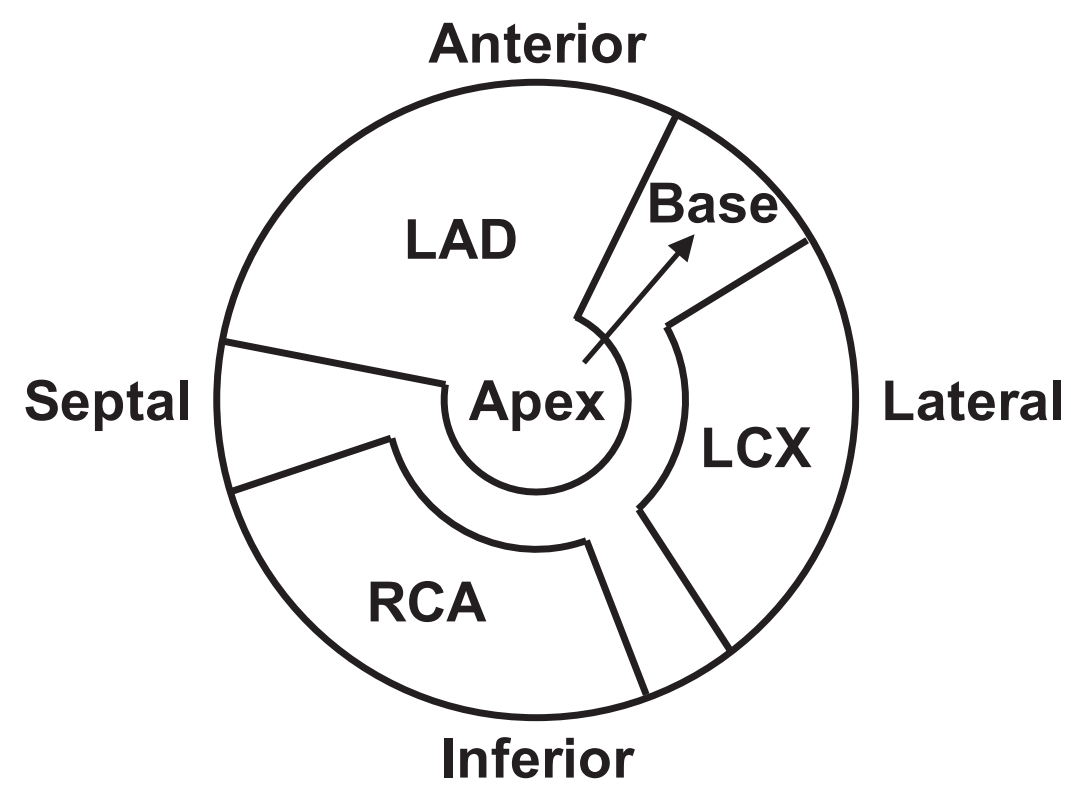

Figure 5.4: Diagram showing the coronary arteries, landmarks, and anatomical directions corresponding to the areas of a polar map. LAD: left anterior descending artery; LCX: left circumflex artery; RCA: right coronary artery.

\subsection{Patient data}

We collaborated with the Cardiac PET Centre at the UOHI to study the feasibility of motion-compensated PET imaging using PeTrack. A single positron-emitting external fiducial marker was placed on the upper right abdomen of patients undergoing cardiac PET imaging. ${ }^{4}$ The same model of marker was used as the one described in Section 4.7.1. The initial activity of the marker was $92.5 \mathrm{kBq}(2.5 \mu \mathrm{Ci})$ on November 1, 2013. All patient studies were acquired with the D690 scanner (see Section 4.6.1). The Varian RPM system (see Section 3.1) was used to monitor breathing motion. The raw PET data (including the signal from the PeTrack marker) were analyzed retrospectively.

\footnotetext{
${ }^{4}$ This was done as part of the clinical quality assurance program of the Cardiac PET Centre.
} 


\subsection{Respiratory gating using PeTrack}

Two studies were selected to provide a proof of concept of PeTrack for respiratory gating in PET imaging. In the first study (Anon4246), a patient underwent a ${ }^{82} \mathrm{Rb}$ stress study during which carbon dioxide $\left(\mathrm{CO}_{2}\right)$ was administered to the patient three minutes before tracer injection and continued three minutes post injection. $\mathrm{CO}_{2}$ causes patients to breathe deeply and activates a physiological response which causes physical stress. The second study (Anon4886) was a typical cardiac PET scan, but exhibited baseline shift of the breathing signal.

\subsubsection{Respiratory triggers from PeTrack}

As in the previous chapter, PeTrack was used to produce the motion trace of the marker from the list mode files of the D690. The marker was located every $0.5 \mathrm{~s}$. Figure 5.5 shows the motion trace of the marker in each of the three anatomical directions for both studies. 


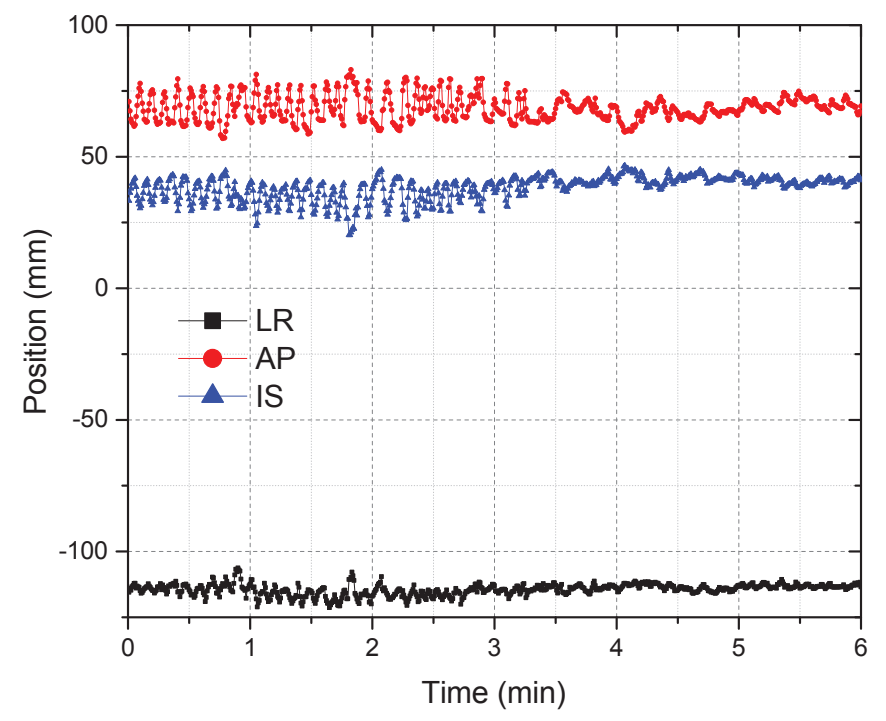

(a) Anon4246

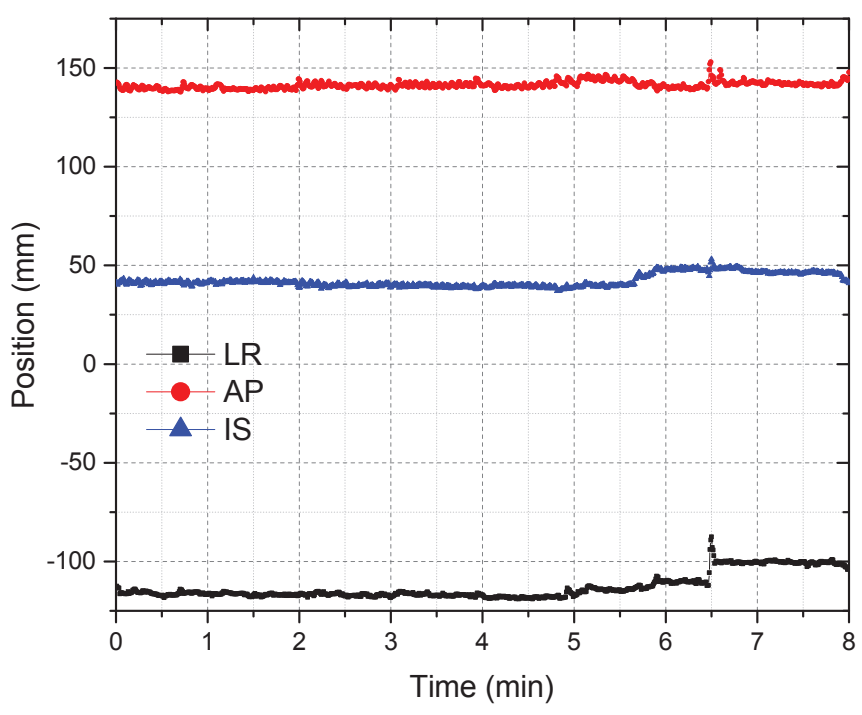

(b) Anon 4886

Figure 5.5: Motion trace of the marker in each direction for each study. Anon4246 was administered $\mathrm{CO}_{2}$ three minutes before tracer injection, until three minutes post injection. Anon4886 received no $\mathrm{CO}_{2}$. 
Respiratory motion is visible on both the AP and IS directions. In particular, the effect of the $\mathrm{CO}_{2}$ on Anon4246 is apparent in the AP and IS directions before the 3minute mark, at which point $\mathrm{CO}_{2}$ administration stopped. The LR direction does not exhibit very large respiratory motion. Since the amplitude of motion in the AP and IS directions was small for Anon4886 and for Anon4246 (after the 3-minute mark), the value of the position of the marker of the marker at time $t$ in the AP direction, $r_{A P}(t)$, and in the IS direction, $r_{I S}(t)$, were added in quadrature to produce a trace $r(t)$ with a larger amplitude of motion:

$$
r(t)=\sqrt{r_{A P}(t)^{2}+r_{I S}(t)^{2}}
$$

This trace is shown on Figure 5.6. The inset of Figure 5.6(b) clearly shows respiratory motion, though the amplitude is only a few millimetres at times.

The temporal location of the peaks in the AP/IS trace of each study were determined using an automated peak finder feature from the OriginPro software (OriginLab Corporation, Northampton, MA, USA). The peaks were identified as the local maxima among groups of four consecutive data points. The results were visually assessed for missed or erroneous peaks, as judged appropriate. ${ }^{5}$ The original list mode files from the D690 were stripped of their RPM-generated respiratory triggers. New respiratory triggers based on the location of the peaks from the PeTrack motion traces were generated and inserted in the list mode files.

\footnotetext{
${ }^{5}$ Note that the motion traces recorded by the RPM system and used to generate the RPM respiratory triggers are not reviewed by UOHI staff after acquisition.
} 


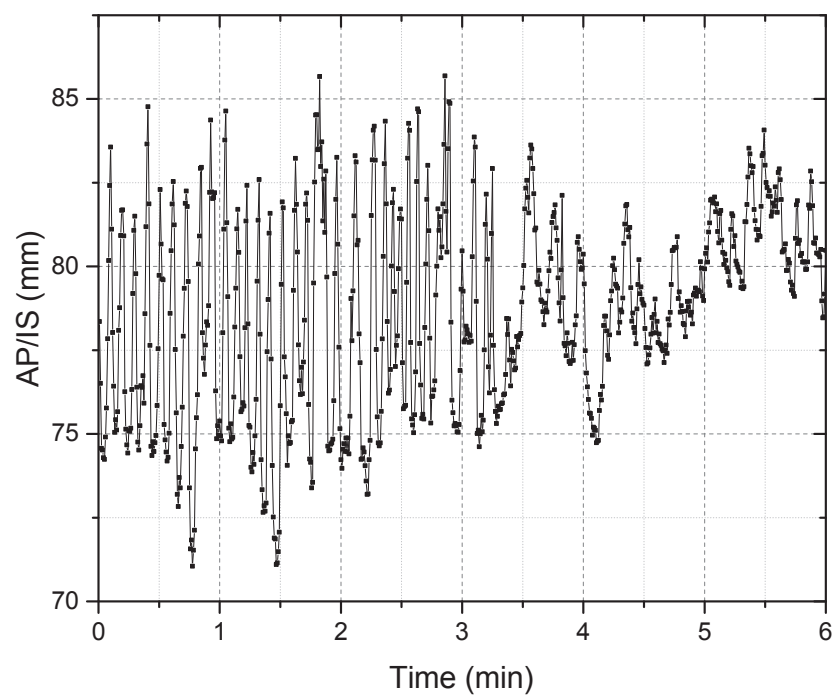

(a) Anon4246

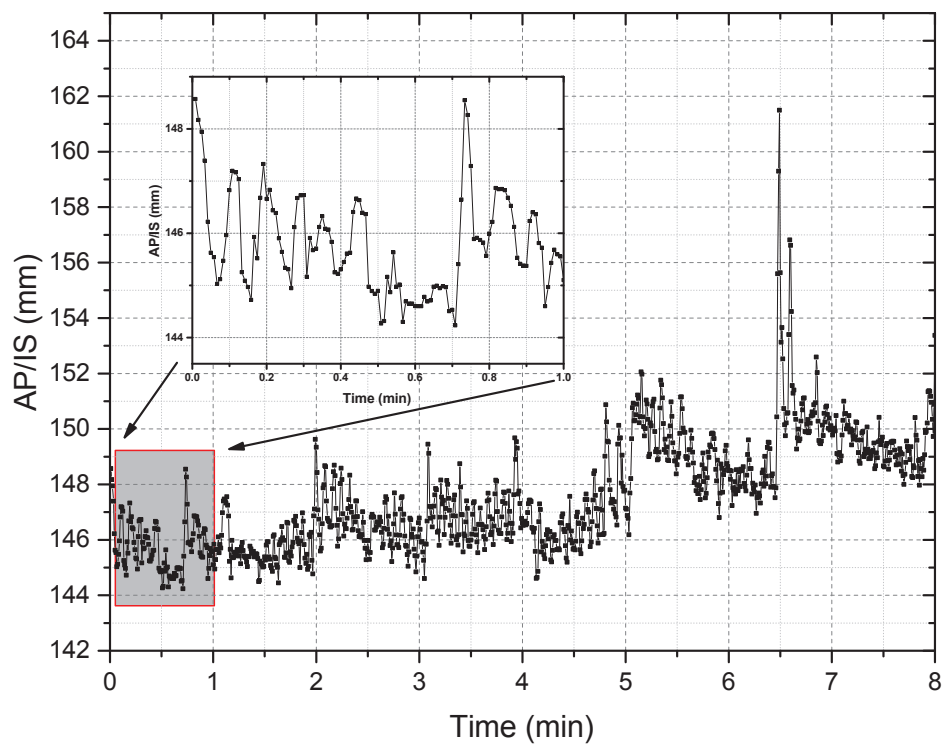

(b) Anon 4886

Figure 5.6: Motion trace of the marker in each direction for each study. Anon 4246 was administered $\mathrm{CO}_{2}$ three minutes before tracer injection, until three minutes post injection. This explains the change in breathing amplitude at the 3 -minute mark. Anon4886 received no $\mathrm{CO}_{2}$. 


\subsubsection{Comparison of gating using RPM and PeTrack}

The original list mode files which included the RPM respiratory triggers, as well as the modified list mode files with the new PeTrack triggers, were fed back to the GE Discovery $690 \mathrm{PET} / \mathrm{CT}$ scanner for reconstruction into gated series.

The number of triggers, their temporal location, and the breathing interval for both the RPM system and PeTrack were quantified. The breathing interval is defined as the time difference between two consecutive respiratory triggers and corresponds to the duration of one full breath cycle.

In addition, the polar maps of the perfusion of the left ventricle of the heart were generated using Corridor 4DM ${ }^{6}$, a cardiac PET perfusion analysis software. The polar maps were assessed visually and qualitatively.

\subsubsection{Results}

Figure 5.7 shows the distribution of the breathing intervals of the two gating systems for both studies. Table 5.1 lists the size, the average, and the standard deviation of each distribution. The distribution of PeTrack triggers is not obviously different from the distribution of RPM triggers. A two-sample Kolmogorov-Smirnov test ${ }^{7}$ yields that the distributions of triggers from the RPM and from PeTrack do not differ significantly at the 0.01 level, for either of the studies.

\footnotetext{
${ }^{6}$ INVIA, Ann Arbor, Michigan, USA

${ }^{7}$ The two-sample Kolmogorov-Smirnov test is a nonparametric statistical test. It determines if the empirical distribution function of the two samples differ.
} 


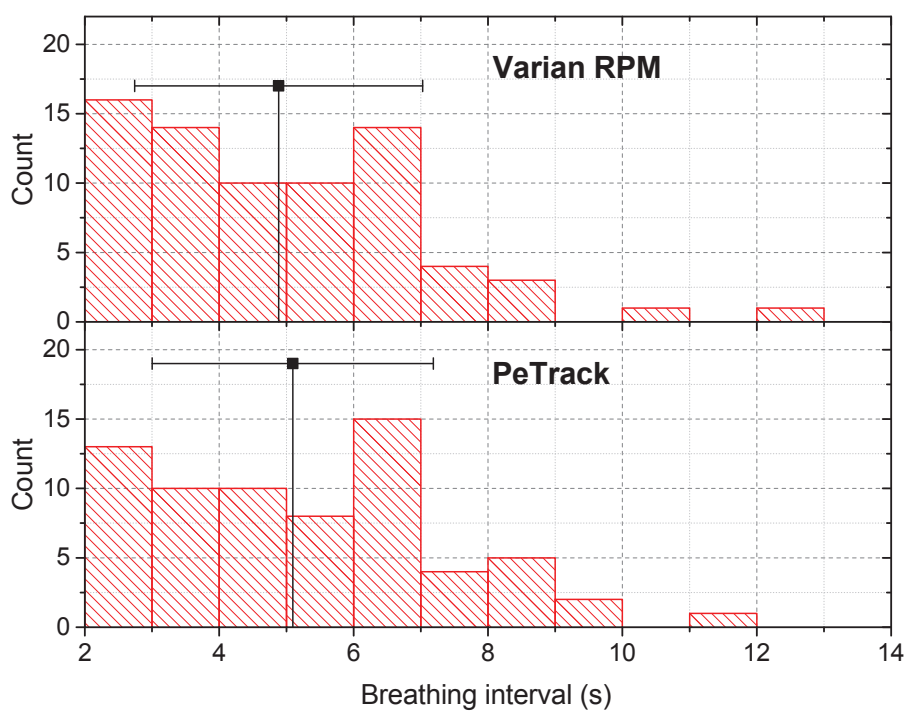

(a) Anon4246

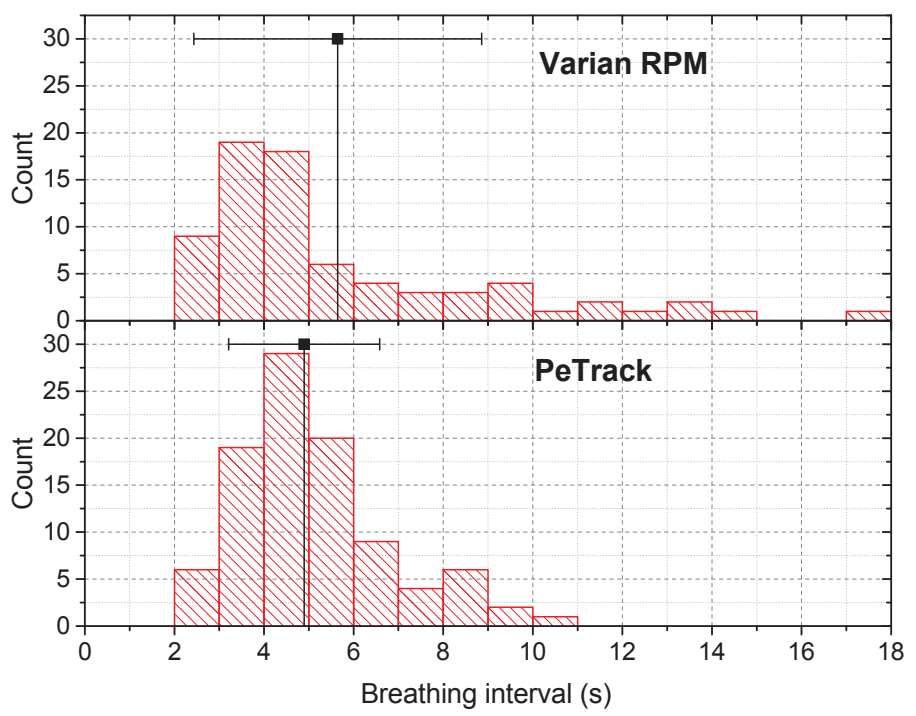

(b) Anon4886

Figure 5.7: Histograms of the distribution of the breathing interval for both gating systems. 
Table 5.1: Statistics on the respiratory triggers from the Varian RPM system and from PeTrack (after user review). Note that user intervention for PeTrack added approximately 15 triggers to Anon4246 and 5 to Anon4886. Those additional triggers had a small effect on the overall statistics of the triggers.

\begin{tabular}{ccccc}
\hline \hline Study & \multicolumn{2}{c}{ Number of triggers } & \multicolumn{2}{c}{ Average breathing interval (s) } \\
& Varian RPM & PeTrack & Varian RPM & PeTrack \\
\hline \multirow{2}{*}{ Anon4246 } & 73 & 69 & $4.9 \pm 2.1$ & $5.0 \pm 2.1$ \\
Anon4886 & 74 & 87 & $5 \pm 3$ & $4.9 \pm 1.7$ \\
\hline \hline
\end{tabular}

Figure 5.8 and Figure 5.9 compare graphically the PeTrack triggers (i.e. the peaks of the motion trace; short red dashes) with the triggers of the RPM system (blue vertical dashed lines). In this study, PeTrack triggers on the opposite phase of the breathing cycle (end inhale) relative to the RPM system (end exhale) because the peaks were more clearly defined at end inhale. However, the extraction of the breathing interval to generate the respiratory triggers should be the same within the precision and accuracy of each system. Triggering at a different phase of the cycle will not affect the perfusion analysis.

Figure 5.10 shows the polar maps of the average perfusion of the LV for Anon4246 and Anon4886. Comparison of the perfusion between the RPM and PeTrack gating reveals minimal differences for both Anon4246 and Anon4886. 


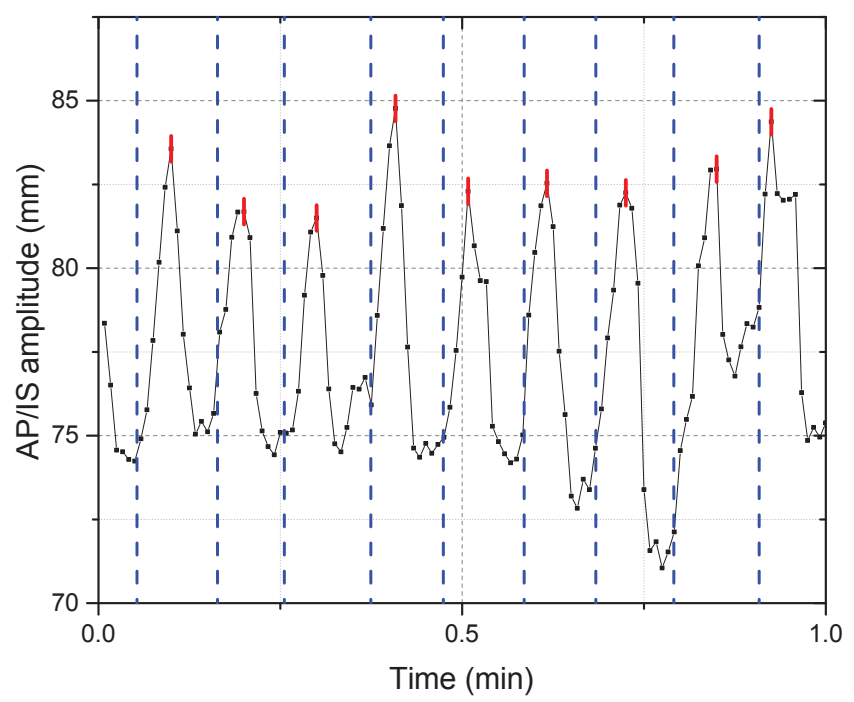

(a) Early in the scan

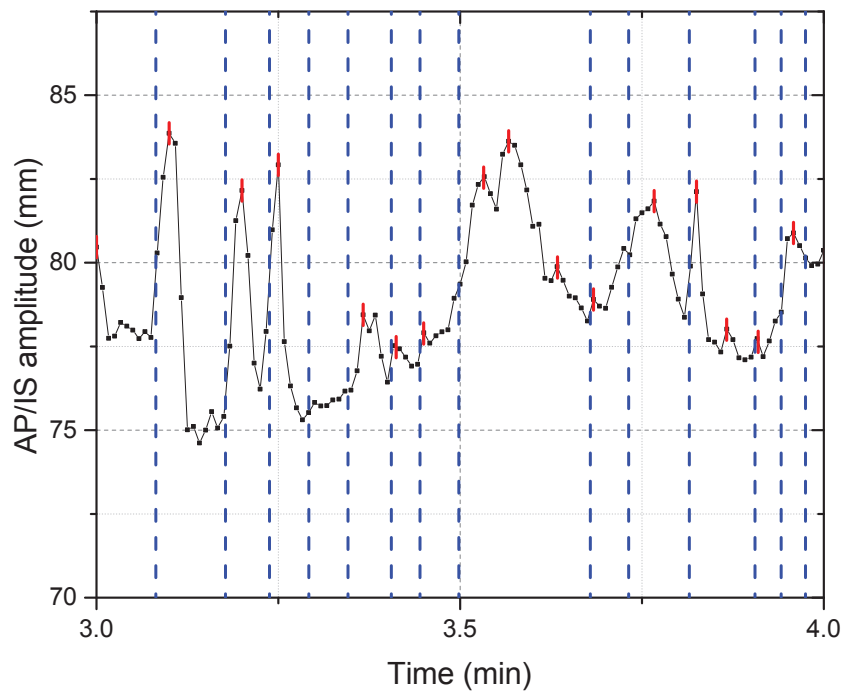

(b) Late in the scan

Figure 5.8: Comparison of the PeTrack respiratory triggers (identified as the peaks of the motion trace; short red vertical dashes) and the RPM triggers (blue vertical dashed lines) for Anon4246. 


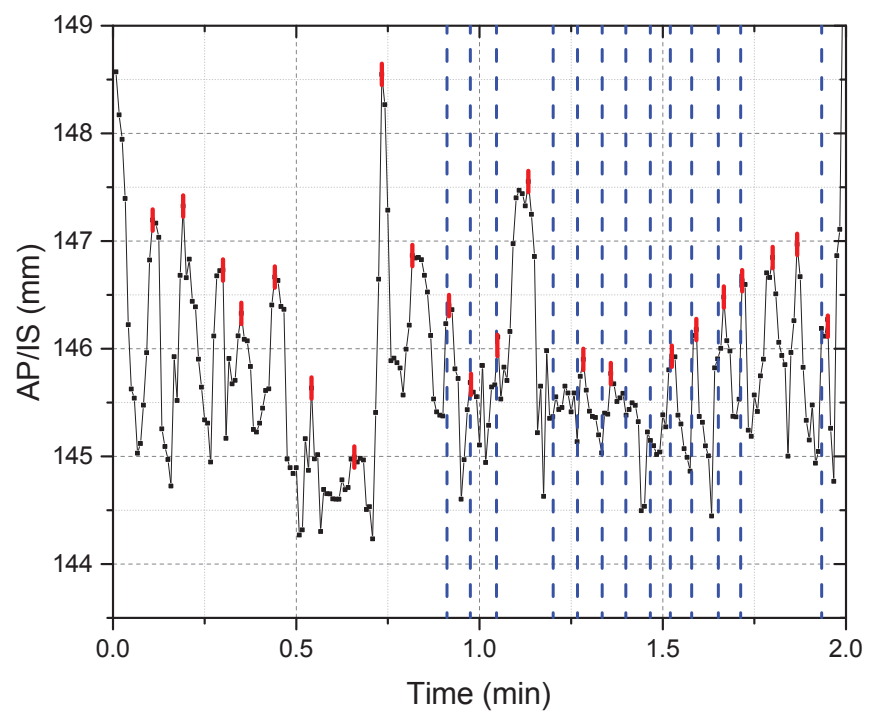

(a) Early in the scan (the RPM only starts recording triggers around the 1-minute mark by protocol)

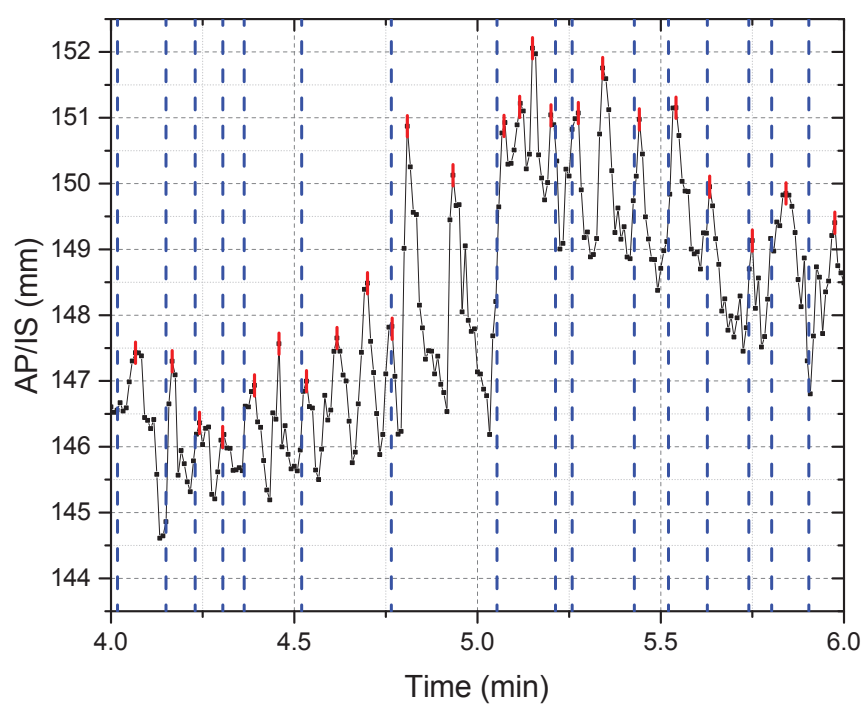

(b) Late in the scan

Figure 5.9: Comparison of the PeTrack respiratory triggers (identified as the peaks of the motion trace; red vertical dashes) and the RPM triggers (blue vertical solid lines) for Anon4886. 


\section{Perfusion}

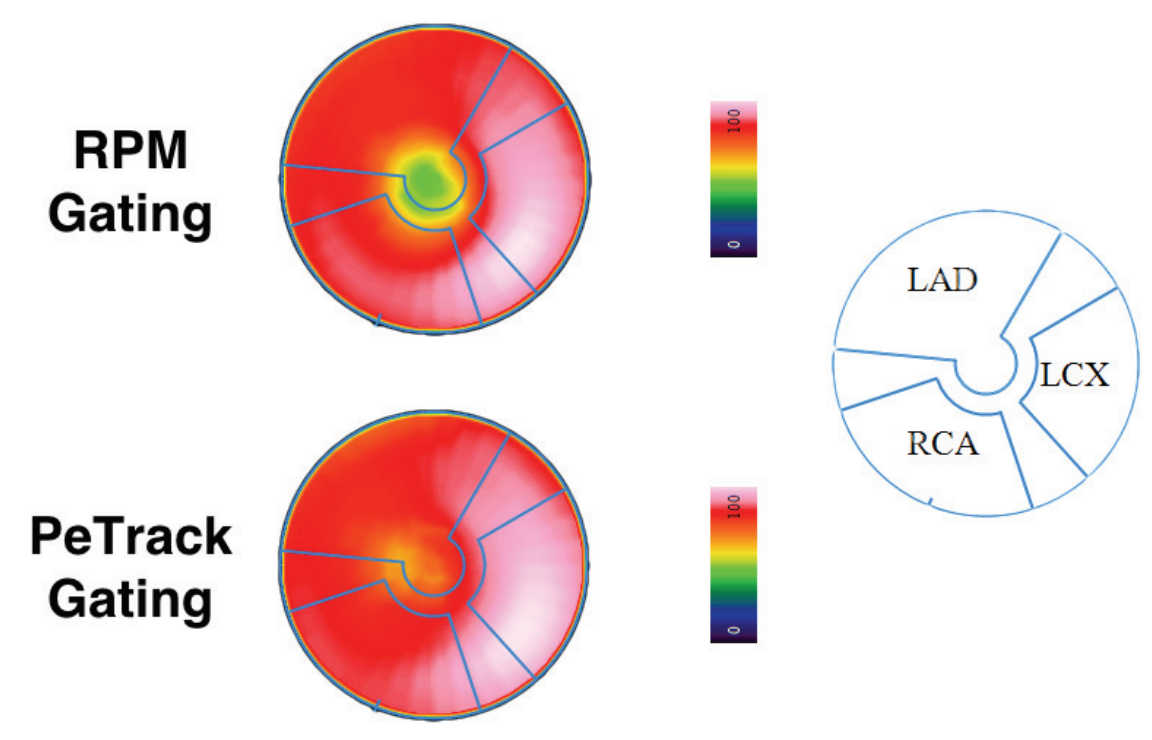

(a) Anon4246

\section{Perfusion}

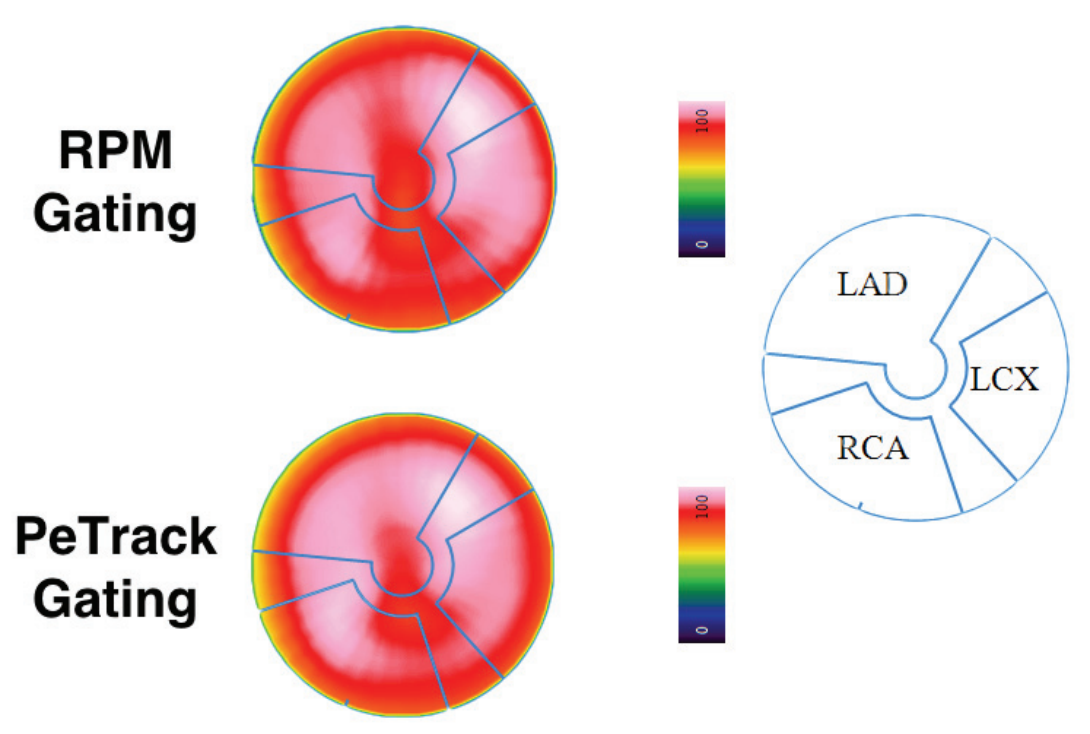

(b) Anon4886

Figure 5.10: Comparison of the polar maps of the average perfusion of the LV for the respiratory gated series of Anon4246 and Anon4886. 


\subsubsection{Discussion}

The two respiratory gating studies provide evidence that PeTrack can be used for respiratory gated cardiac PET imaging and provide similar or better results than the typical Varian RPM system used. Typically, the breathing interval of healthy adults is in the range of 3 to 5 s. [133] Respiratory triggers from PeTrack show similar breathing rates, even though patients undergoing cardiac PET imaging may not necessarily be healthy. On the other hand, the RPM distribution of study Anon4886 seems wider than expected.

A closer look at Figure 5.9 reveals that the RPM system misses triggers from time to time. Missing consecutive triggers tend to increase the breathing interval, which is reflected in the distribution of the breathing interval. To avoid this problem with PeTrack, the motion traces and the detected peaks were visually reviewed. Though this adds extra work for the PET imaging staff, some level of user intervention is typically needed to review results of automatic peak detection methods given the large variability in breathing patterns. [134,135]

Anecdotally, the UOHI team reports that the RPM system misses triggers when the breathing pattern of a patient exhibits a baseline drift. Figure 5.8(b) and Figure 5.9(b) seem to support this assertion. Baseline shifts in patient breathing patterns are a common occurrence in PET imaging. [80,111,136-138] Others also report that this may cause errors when binning the data into the appropriate respiratory gate. [138] PeTrack has an advantage over the RPM system with respect to its robustness to baseline shifts. As long as the marker stays within the FOV of the scanner, baseline shifts have no effect on the performance of PeTrack. On the other hand, noise in the PeTrack trace may make peak identification difficult, especially in cases where the amplitude of motion of the marker is small. However, Anon4886 provides 
compelling evidence that even motion with amplitude on the order of $1 \mathrm{~mm}$ can be resolved by PeTrack and used to generate respiratory triggers.

Despite the user intervention to review the triggers, PeTrack still offers numerous advantages over RPM gating. As outlined in Section 4.8, (i) the PeTrack data is intrinsically synchronized with the PET data, (ii) no spatial co-registration is required, (iii) it is a pure software solution, excluding the need for fiducial markers, and (iv) the raw motion data are stored within the list mode files, which are usually archived and not overwritten (as in the case of the RPM motion files).

This proof of concept was performed with time based gating to compare it to the gating provided with the RPM system and the D690 scanner. This type of time based gating uses eight bins of equal sizes between each recorded respiratory trigger and is commonly used in commercial systems [139]. It has been shown that this type of gating performs worse than amplitude based gating. [111,137,138] In amplitude gating, the events are binned into gates based on the magnitude of the displacement of the motion or surrogate signal. This typically captures the motion better than time based gating because of the large variability in amplitude and period of breathing patterns. In time based gating, asymmetric breathing patterns (e.g. if the exhale phase is particularly long) can cause most of the motion to be binned into the first gates. After reconstruction, this leads to blurred images in the early gates and little motion in the later gates. Time based gating can underestimate the physical motion of the heart by up to $50 \%$. [140]

Consequently, amplitude based gating with PeTrack should be explored in future work. Given the accuracy and repeatability of PeTrack, amplitude gating is expected to be feasible. In particular, Anon4246 would be a good candidate study for amplitude gating given the regular and large amplitude of the motion, especially during $\mathrm{CO}_{2}$ administration (see Figure 5.5(a)). A case study such as Anon4886 might prove more challenging due to the smaller amplitude of motion. For example, if eight equal size 
gates are used for a motion trace with peak-to-peak amplitude of $10 \mathrm{~mm}$, then each gate has a height of $1.25 \mathrm{~mm}$. The binning of the events into the appropriate gates will be noisy due to the accuracy and repeatability of PeTrack. However, Dawood et al. have suggested a method to determine the optimal number of amplitude based gates that should be used in cardiac PET imaging, based on the measured displacement of the heart. [140]

\subsection{Motion correction using PeTrack}

This section will present a proof of concept application in which the motion trace of a marker is used to correct translational motion from the raw PET list mode data on an event-by-event (EBE) level. The main idea is to translate each detected coincidence event to where it would have been detected if no motion had occurred. Four studies were selected in which significant motion was observed: the two dynamic phantom studies from Chapter 4, the study Anon4886 from Section 5.5, and another dynamic cardiac PET imaging study (Anon3512). In addition, the motion correction method was also applied to the data from the static phantom scan. This was done to verify that motion correction of a static scan results in images that are equivalent to the images reconstructed with non-motion-corrected data.

\subsubsection{Event-by-event motion correction}

The initial location of the marker at the beginning of the scan, $\mathbf{r}_{r e f}$ was used as a reference. The initial location was determined either from the attenuation correction CT scan or from the first frame of the dynamic PET series. At each localization time $t_{n}$, a motion correction (MC) vector was calculated as:

$$
\Delta_{M C}\left(t_{n}\right)=\mathbf{r}_{r e f}-\mathbf{r}\left(t_{n}\right)
$$




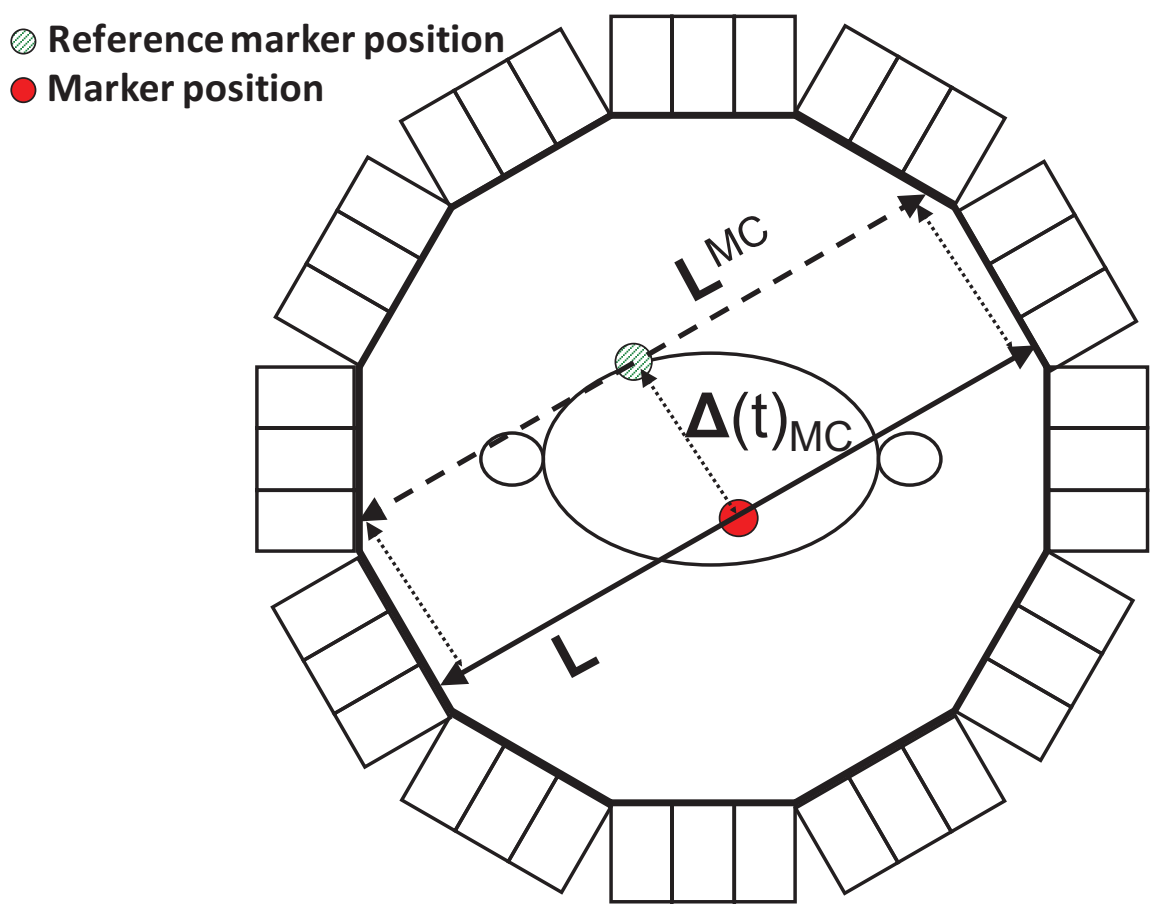

Figure 5.11: Illustration of the motion correction of events. The motion correction vector at time $t, \boldsymbol{\Delta}_{M C}(t)$, is defined as the difference between the reference marker position and the current marker position. The coincidence event $\mathbf{L}$ gets transformed to $\mathbf{L}^{M C}$.

where $\mathbf{r}\left(t_{n}\right)$ is the location of the marker at time $t_{n}$. The motion correction vector at any time $t_{n}<t<t_{n+1}, \boldsymbol{\Delta}_{M C}(t)$, was interpolated linearly from $\boldsymbol{\Delta}_{M C}\left(t_{n}\right)$ and $\boldsymbol{\Delta}_{M C}\left(t_{n+1}\right)$. Each coincidence event in the list mode file was converted from list mode format to Cartesian format by using the known geometry of the scanner (see Table 4.1) and the known indexing convention of detector elements. The events were corrected for motion using:

$$
\mathbf{L}^{M C}=\mathbf{L}+\boldsymbol{\Delta}_{M C}(t)
$$

where $\mathbf{L}^{M C}$ is the motion-corrected coincidence event recorded at time $t$ and $\mathbf{L}$ is the original non-MC coincidence event. Figure 5.11 illustrates the motion correction of events. 
Once an event has been corrected for motion, it is converted back into the GE list mode format so that it can be passed to the D690 for image reconstruction.

\subsubsection{Results}

\section{Phantom studies}

The non-MC and MC data from the three phantom scans (see Section 4.7) were reconstructed into static and dynamic series. Figure 5.12 shows a comparison of a sample coronal slice of the static series for each scan. Similarly, Figure 5.13 and Figure 5.14 show sample slices along the short axis and horizontal long axis of the heart, before and after motion correction for all three scans. Figure 5.13 demonstrates that a uniform perfusion is expected in the walls of the heart insert, in the absence of motion. Before motion correction, both Scans \#2 and \#3 show visible blurring and reduced perfusion in the IS direction (see Figure 5.14(a) and Figure 5.14(b)). After motion correction, Scan \#2 is notably similar to the static reference. The original blurring in Scan \#3 is also greatly reduced after motion correction. The decrease in intensity with respect to the static scan is simply explained by the loss of events. As the phantom drifted in the inferior direction during Scan \#3, the heart insert came partially out of the FOV. A large fraction of events produced during that time were completely missed by the scanner. 


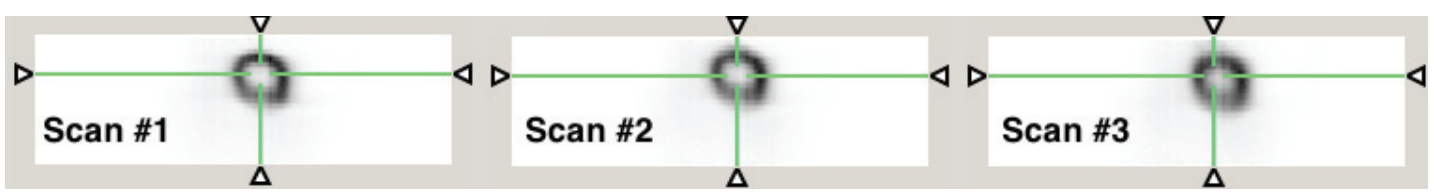

(a) non-motion-corrected.

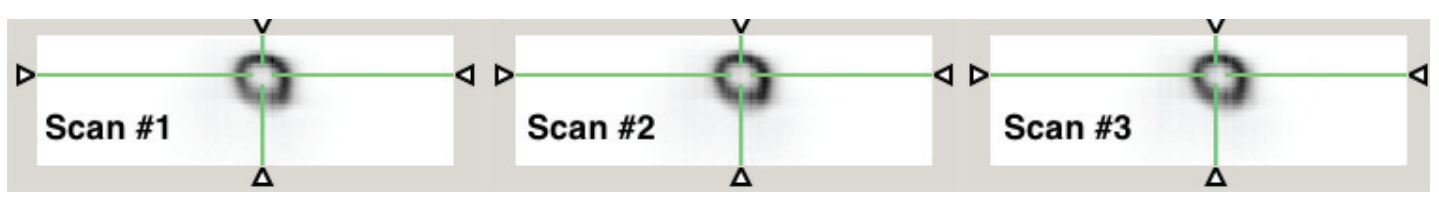

(b) motion-corrected

Figure 5.12: Sample coronal slice of the non-dynamic series for each phantom scan. Scan \#1 is the reference static scan.

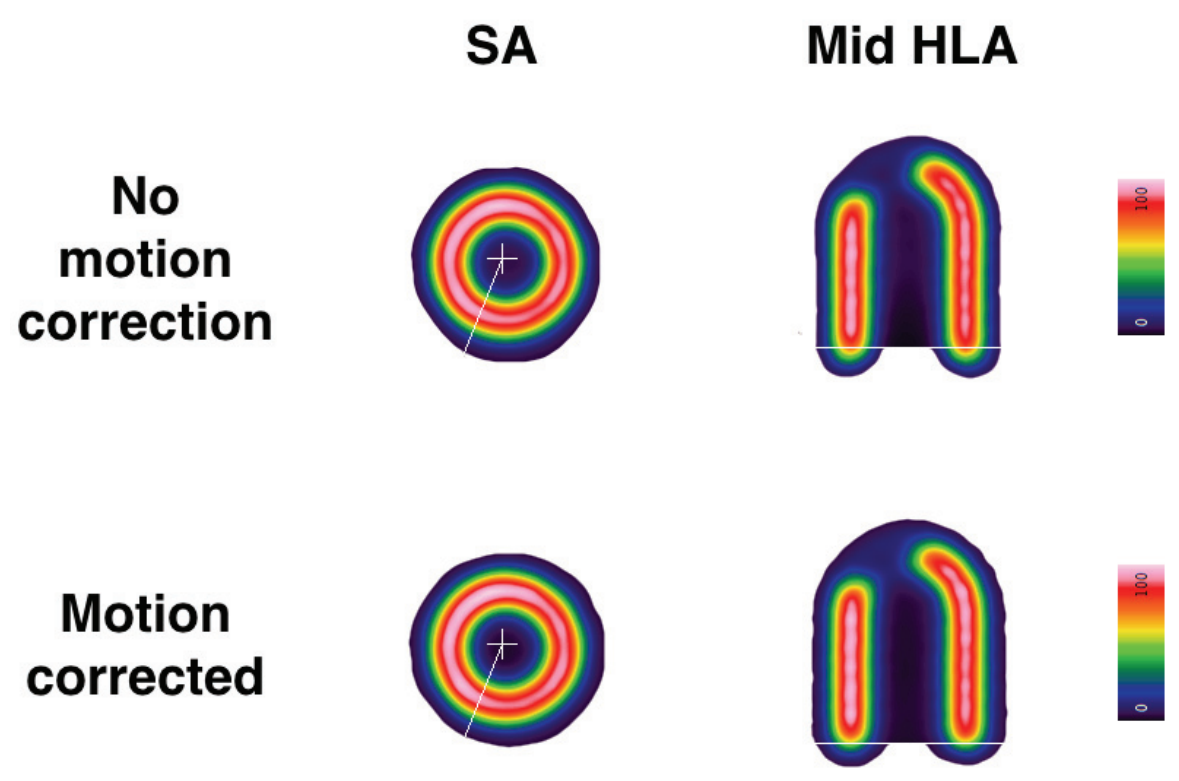

Figure 5.13: Comparison of non-motion-corrected and motion-corrected sample slices along the short axis (SA) and horizontal long axis (HLA) of the heart for the static reference scan (scan \#1). Note the air bubble near the apex of the heart, on the HLA slice. 


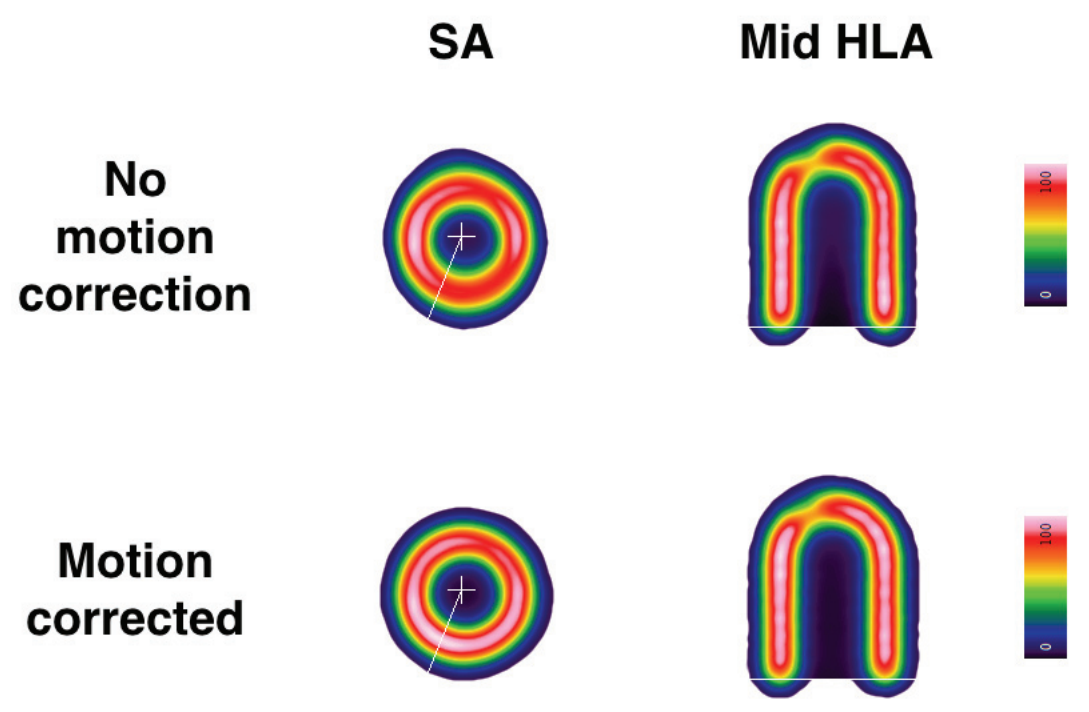

(a) Scan \#2

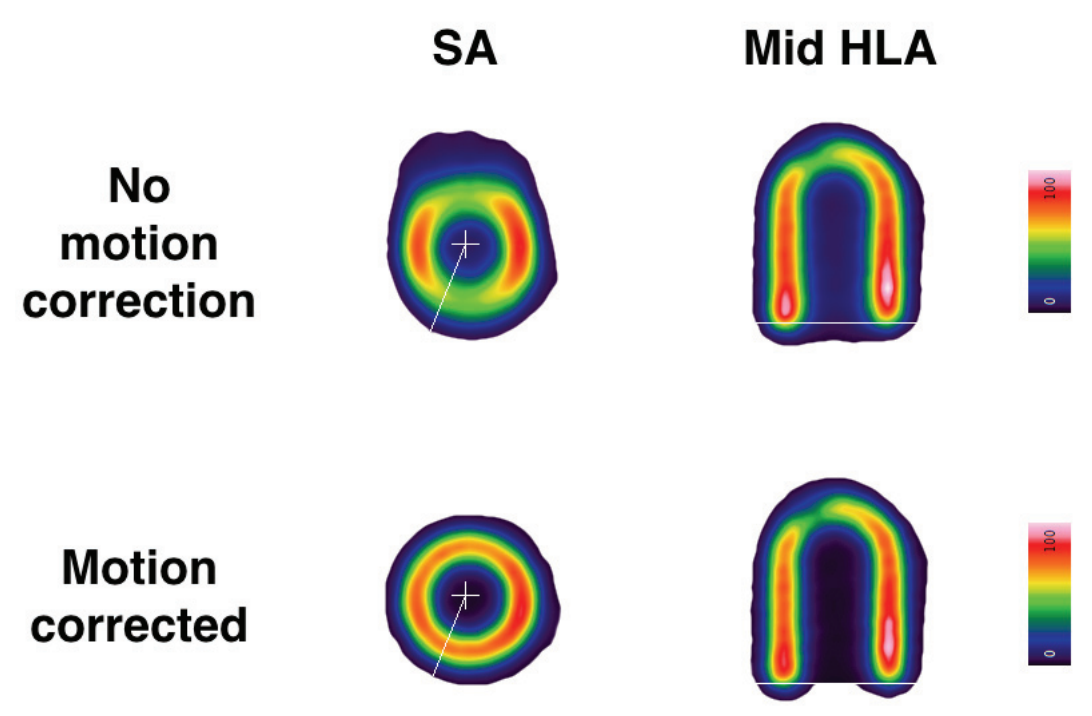

(b) Scan \#3

Figure 5.14: Comparison of non-motion-corrected and motion-corrected sample slices along the short axis (SA) and horizontal long axis (HLA) of the heart for scans \#2 and \#3. Note the air bubble near the apex of the heart, on the HLA slice. 
To assess the effectiveness of the motion correction, a line profile was measured in the IS direction along the vertical line on Figure 5.12. The line profiles are shown on Figure 5.15. The two peaks of the profiles correspond to the inferior and superior walls of the heart insert of the phantom, respectively. The peaks were fitted with a Gaussian model to measure their FWHM. Table 5.2 summarizes the results. 


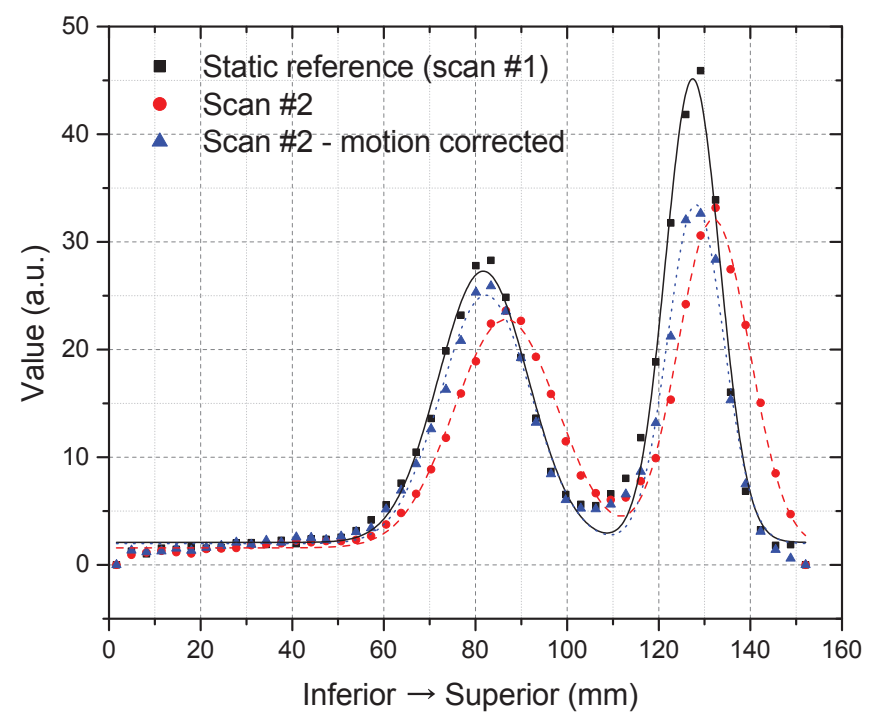

(a) Scan \#2.

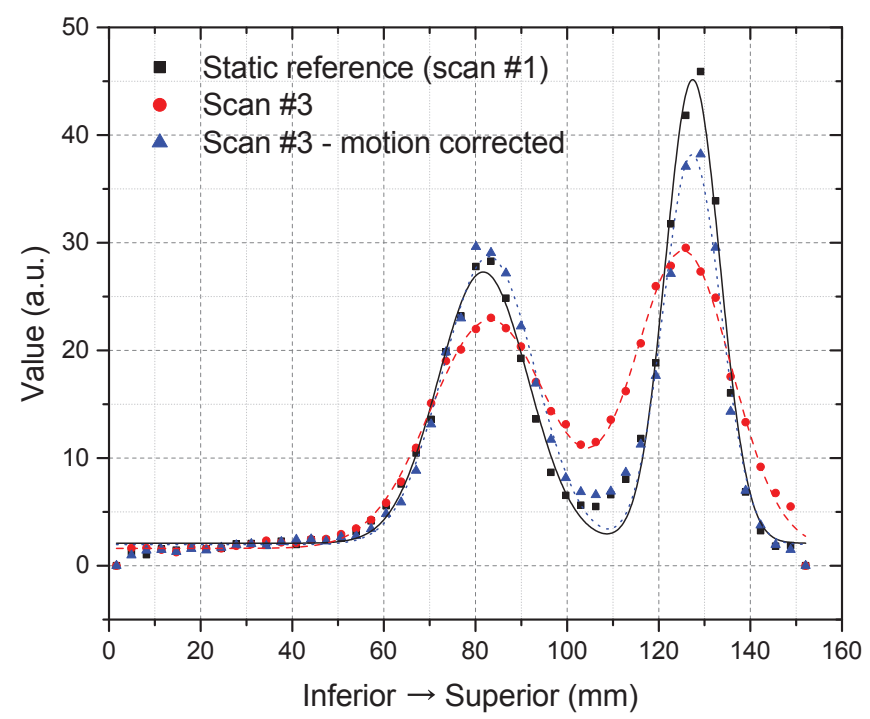

(b) Scan \#3

Figure 5.15: Measured line profiles in the coronal plane along the IS direction. Each peak was fitted with a Gaussian model. Scan \#1 is the reference static scan. 
Table 5.2: FWHM of the fitted peaks of a line profile in the IS direction in the coronal plane for the three phantom scans.

\begin{tabular}{|c|c|c|c|}
\hline & & $\begin{array}{l}\text { FWHM } \\
(\mathrm{mm})\end{array}$ & $\begin{array}{c}\text { Difference } \\
\text { relative to Scan \#1 } \\
(\%)\end{array}$ \\
\hline \multirow{5}{*}{ Inferior peak } & $\begin{array}{c}\text { Scan \#1 } \\
\text { (static reference) }\end{array}$ & $23.1 \pm 0.9$ & $\mathrm{~N} / \mathrm{A}$ \\
\hline & Scan \#2 & $26.7 \pm 0.7$ & 15.6 \\
\hline & $\begin{array}{c}\text { Scan \#2 } \\
\text { motion-corrected }\end{array}$ & $23.2 \pm 0.9$ & 0.2 \\
\hline & Scan \#3 & $30.6 \pm 0.8$ & 32.3 \\
\hline & $\begin{array}{c}\text { Scan \#3 } \\
\text { motion-corrected }\end{array}$ & $23.7 \pm 0.8$ & 2.4 \\
\hline \multirow{5}{*}{ Superior peak } & $\begin{array}{c}\text { Scan \#1 } \\
\text { (static reference) }\end{array}$ & $14.0 \pm 0.4$ & $\mathrm{~N} / \mathrm{A}$ \\
\hline & Scan \#2 & $18.4 \pm 0.4$ & 31.7 \\
\hline & $\begin{array}{c}\text { Scan \#2 } \\
\text { motion-corrected }\end{array}$ & $14.4 \pm 0.5$ & 2.9 \\
\hline & Scan \#3 & $24.8 \pm 0.5$ & 77.1 \\
\hline & $\begin{array}{c}\text { Scan \#3 } \\
\text { motion-corrected }\end{array}$ & $14.8 \pm 0.5$ & 5.6 \\
\hline
\end{tabular}


Although the blurred peaks are not significantly different from the static reference (from a statistical perspective), their FWHM differ from the static reference by as little as $15 \%$ and as much as $77 \%$. After motion correction, this difference is reduced to $5.6 \%$ at most.

A self-consistency test of the motion correction was also performed. If the MC list mode file is fed back to the PeTrack algorithm, the new motion trace of the marker should exhibit very little residual motion. Figures 5.16 and 5.17 show the results of this self-consistency check. As expected, the MC traces are relatively constant; their standard deviation ranges from $0.3 \mathrm{~mm}$ (scans \#2 and \#3, LR direction) to $1.5 \mathrm{~mm}$ (scan \#2, IS direction). The discontinuities in the motion-corrected IS traces of scan \#2 and the fact that the LR and AP traces are unchanged after motion correction will be explained in Section 5.6.3. Note that scan \#3 used the location of the marker on the CT images as a reference for the motion correction, which explains the slight shift between the original and the motion-corrected traces.

Polar maps of the perfusion of the LV of the heart insert were also produced (see Figure 5.18). The polar maps for Scan \#3 were generated with a gated series. Respiratory triggers for Scan \#2 failed to be saved to the list mode. Motion in the IS direction causes reduced perfusion in the inferior area of the non-motion-corrected polar map (see Figure 5.18(a)); after motion correction, the perfusion in the inferior area is more uniform. Similarly, the larger motion during Scan \#3 is reflected in its polar maps. In particular, perfusion is less uniform. Motion correction improves the appearance of the perfusion polar maps, but uniformity is not achieved due to loss of events from the phantom moving out of the FOV. The polar map of wall motion of the LV for Scan \#3 after motion correction displays small residual motion compared to the non-motion-corrected case. The wall motion was measured by an automatic segmentation algorithm. 


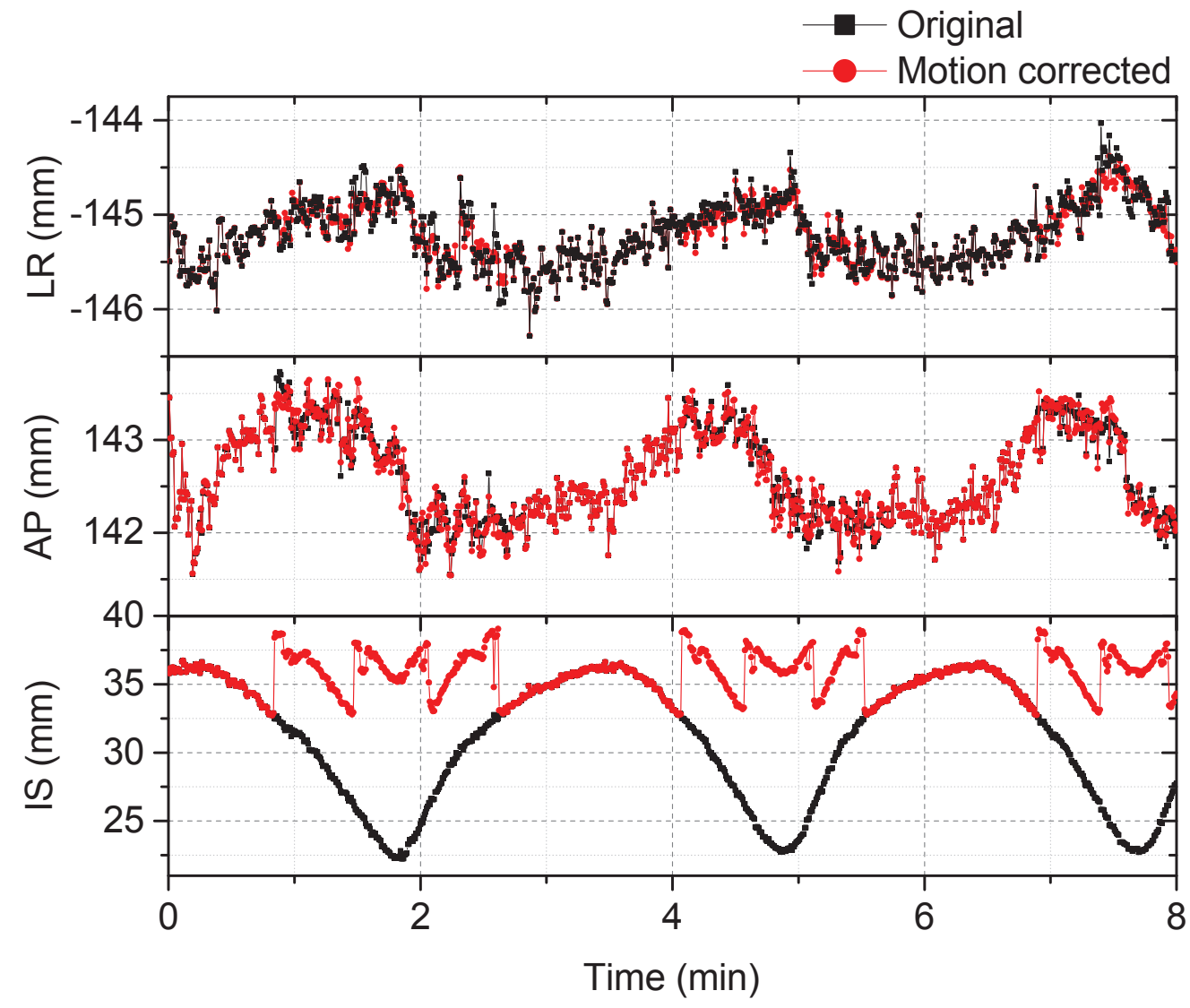

Figure 5.16: Non-MC and MC traces of the marker for phantom scan \#2. Note the unchanged LR and AP traces after motion correction and the discontinuities in the IS direction; those will be explained in Section 5.6.3. 


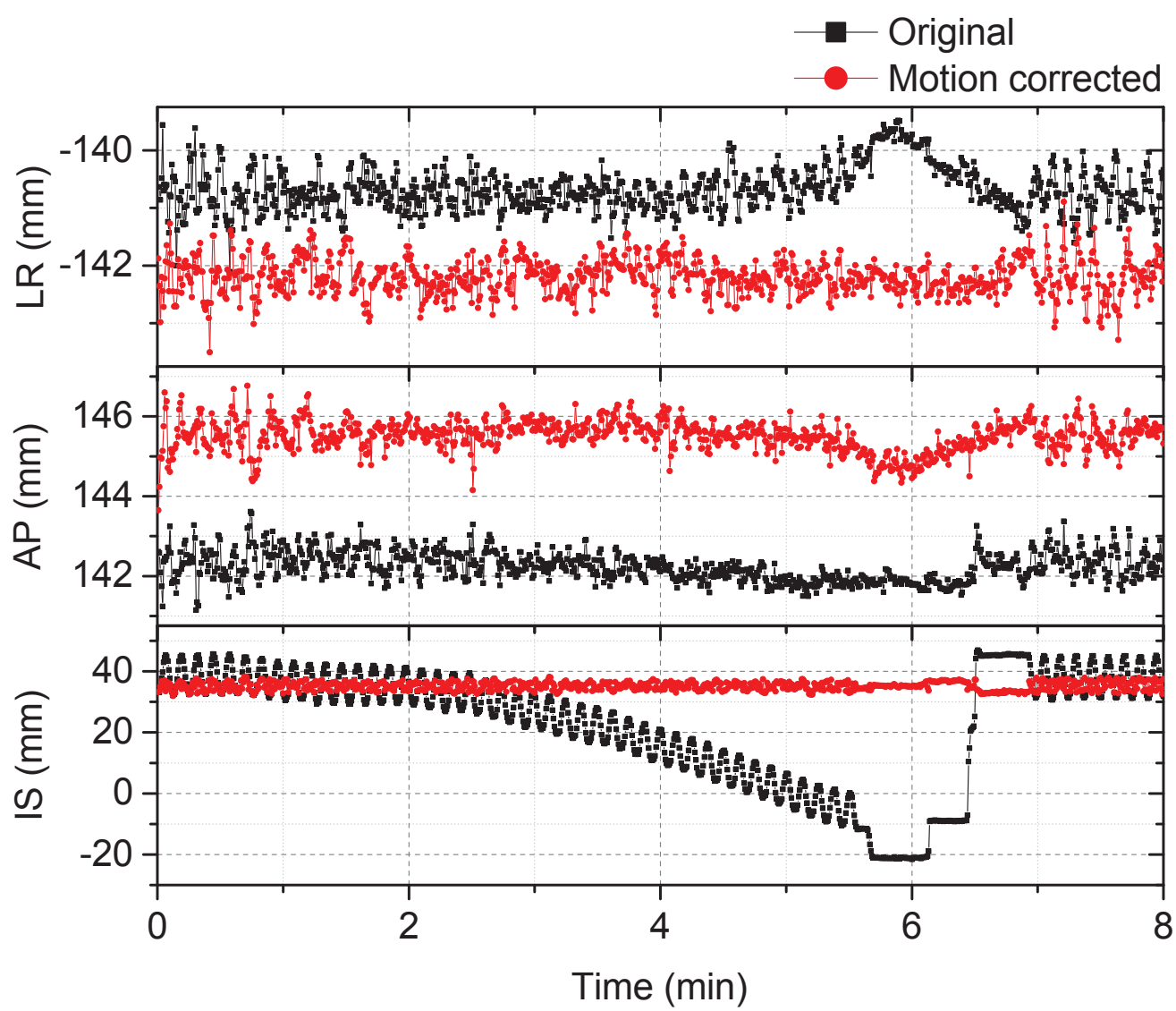

Figure 5.17: Non-MC and MC traces of the marker for phantom scan \#3. The shift between the LR traces and the AP traces before and after motion correction is caused by choosing the reference position of the marker on a CT image. The position of the marker on the CT image was slightly offset from the position of the marker on the PET image. 


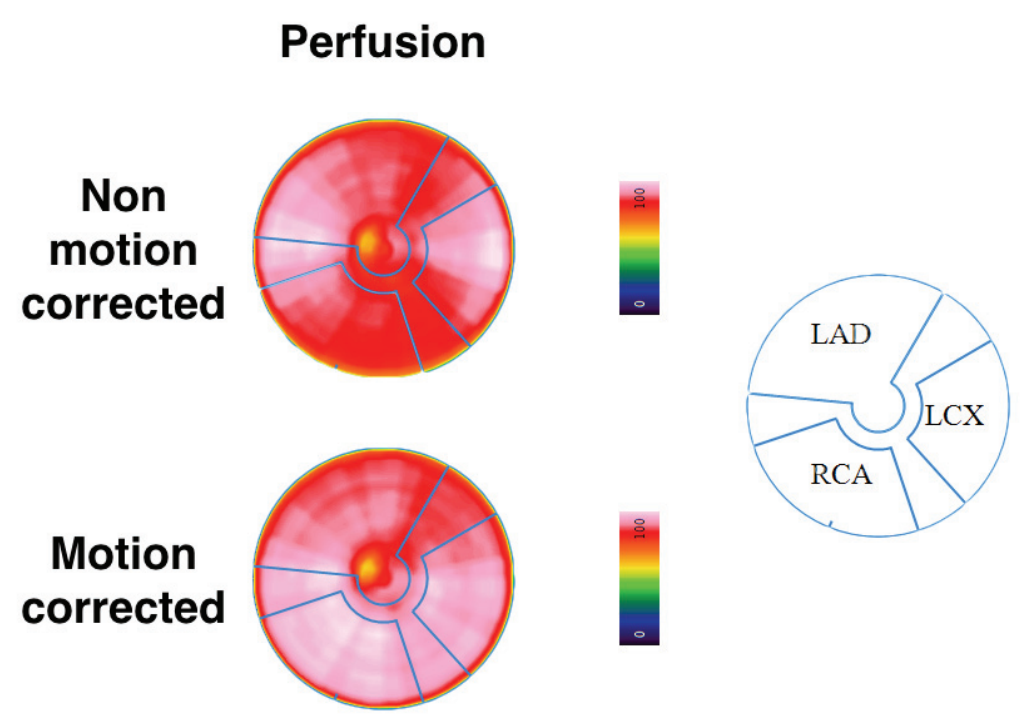

(a) Scan \#2

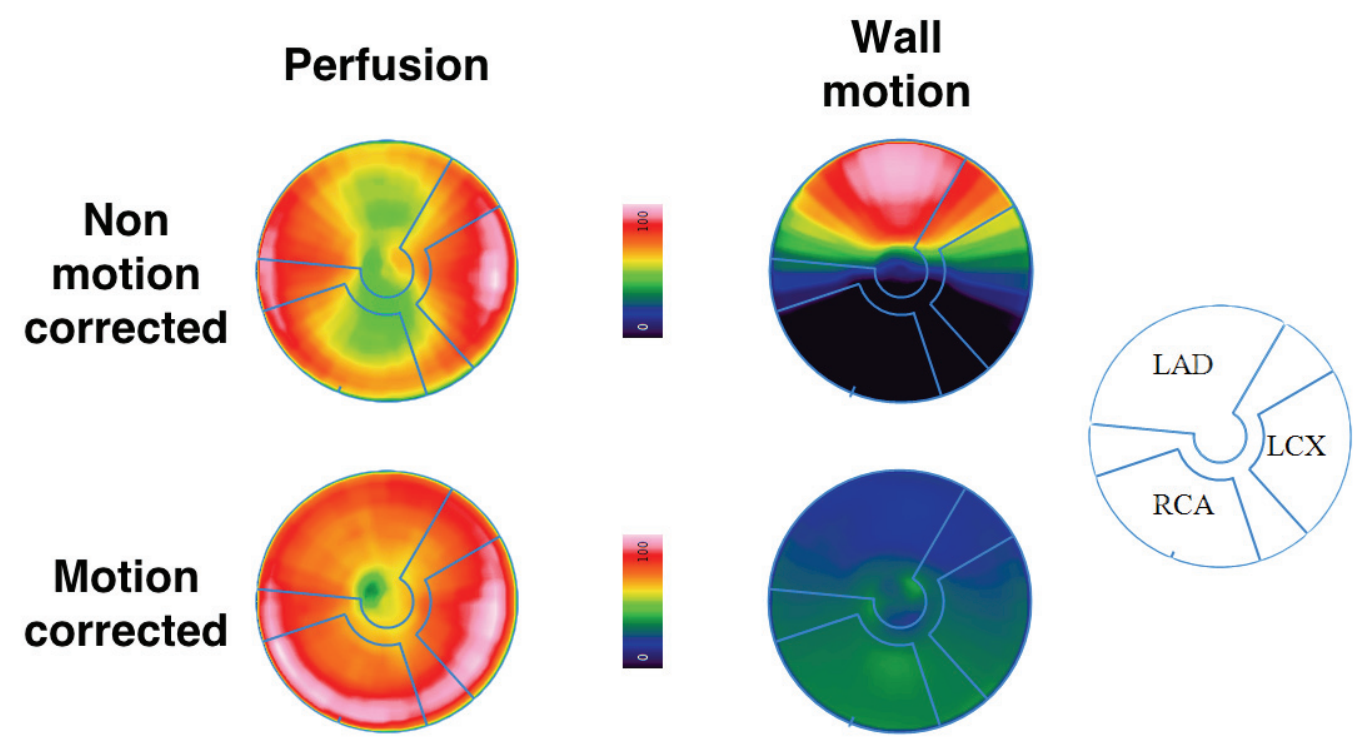

(b) Scan \#3

Figure 5.18: Comparison of the polar maps of the average perfusion of the LV for phantom scans \#2 and \#3. Scan \#3 also includes a polar map of the detected wall motion by an automatic segmentation algorithm. Note that the non-uniformity of the perfusion near the apex of the heart is most likely due to an air bubble in the heart insert (compare to Figure 5.14). 


\section{Anon4886}

The motion trace of Anon4886 (see Figure 5.5(b)) exhibited large motion particularly in the IS direction. After motion correction and running the self-consistency test described in the previous section, the MC traces are motion free (see Figure 5.19), with standard deviations between 0.5 (LR direction) and $1.2 \mathrm{~mm}$ (IS direction).

Static series were also reconstructed. A line profile was measured across the marker, along the LR direction in the transverse plane (see Figure 5.20). Blurring in the LR direction due to patient motion is visible on the non-motion-corrected image and reduced in the motion-corrected one. A Gaussian model was fitted to the profiles (see Figure 5.21). The FWHM was reduced from $23.57 \pm 0.25 \mathrm{~mm}$ to $18.06 \pm 0.11 \mathrm{~mm}$ after motion correction. 


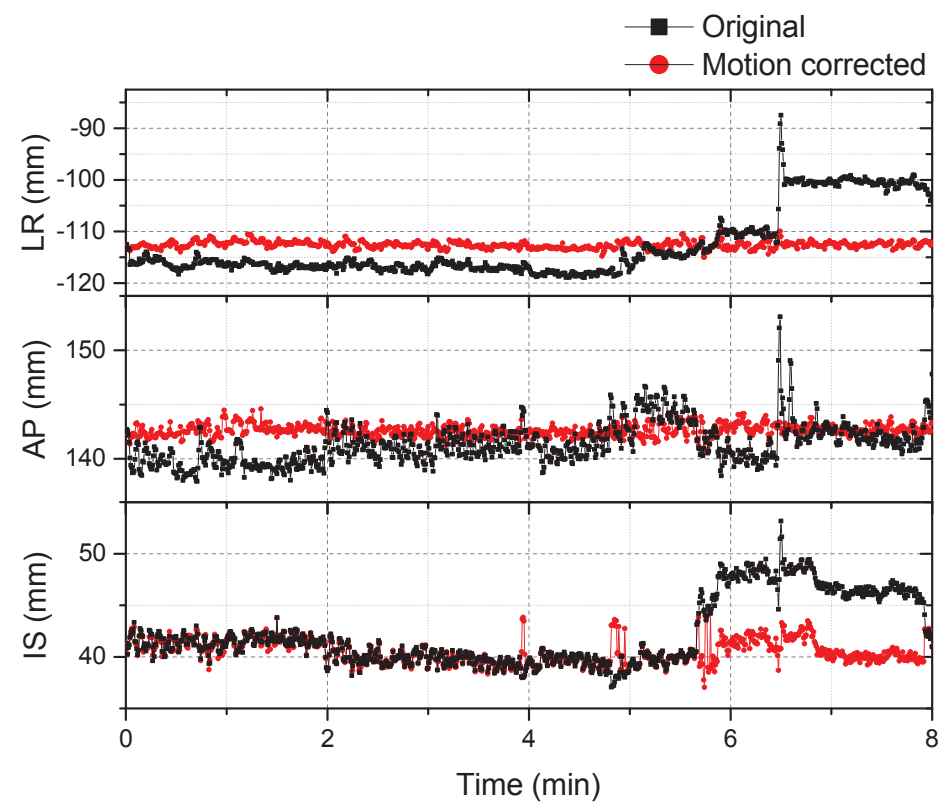

Figure 5.19: Non-MC and MC traces of the marker for Anon4886. 


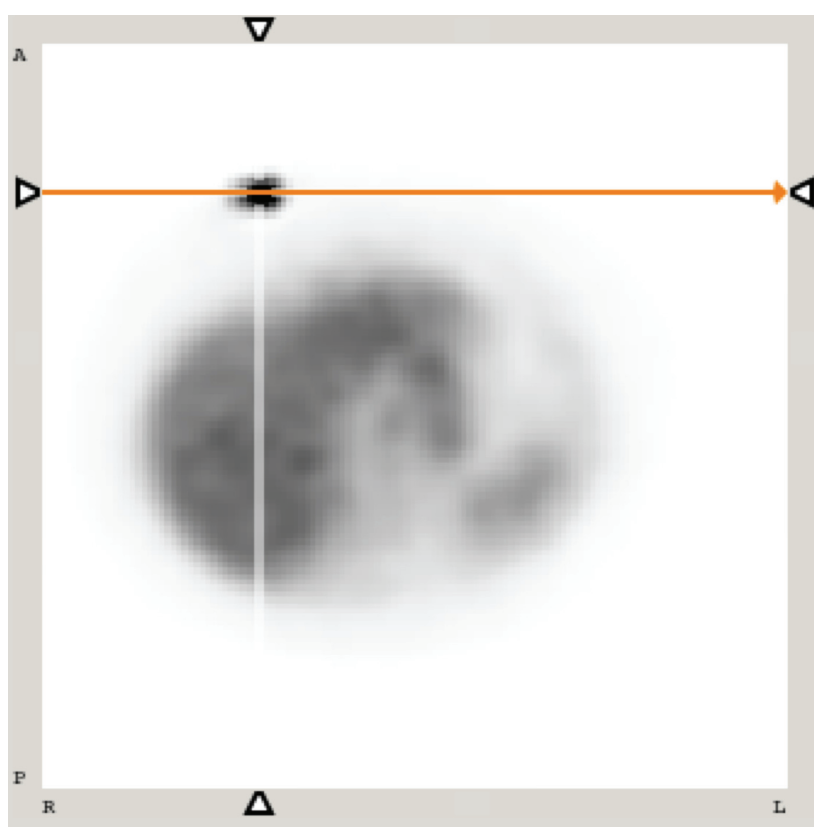

(a) non-motion-corrected

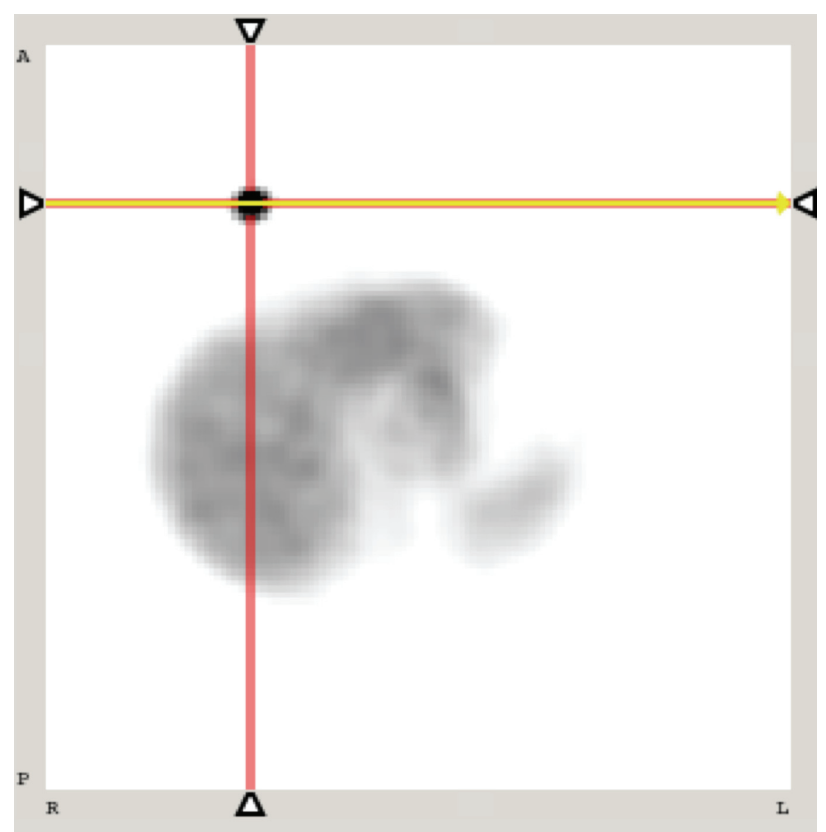

(b) motion-corrected

Figure 5.20: Line profile across the marker and along the LR direction in the transverse plane of Anon4886. The AP direction (vertical line) is not used. 


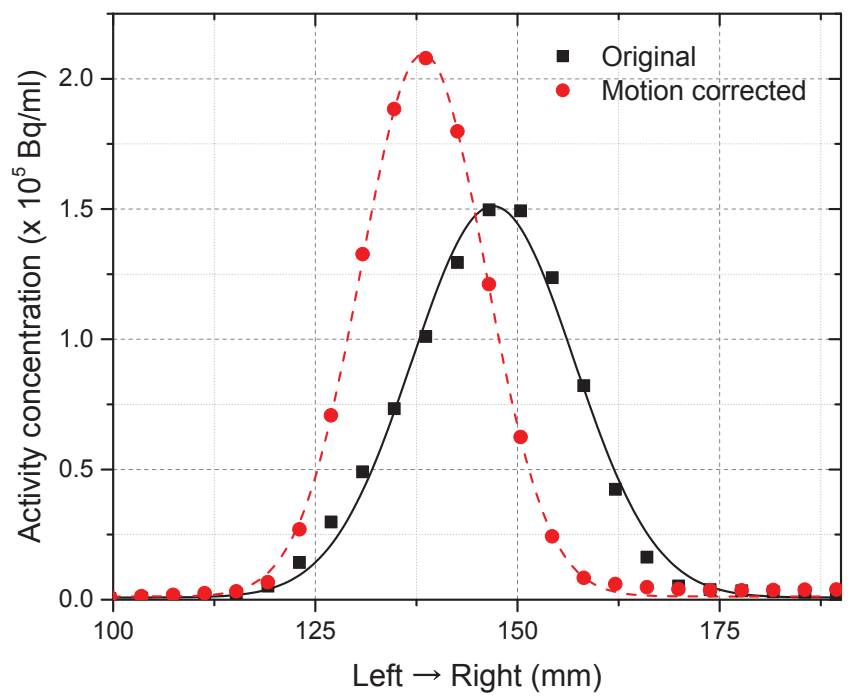

Figure 5.21: Measured line profile across the marker and along the LR direction in the transverse plane of Anon4886. A Gaussian model was fitted to the profile. 


\section{Anon3512}

The final study used to evaluate the feasibility of PeTrack for motion correction was Anon3512. This particular study had very large motion in all three directions (see Figure 5.22). After motion correction, the self-consistency check yielded relatively motion free traces as expected. However, visual assessment of the dynamically reconstructed series (32 frames of duration $15 \mathrm{~s}$ each) demonstrated that large residual motion of the heart was left in the motion-corrected data. Figures 5.23 and 5.24 show the non-MC and MC reconstructed images for a sample transverse slice in the dynamic series. The dynamic series shows that the original motion was a mixture of translation and rotation of the patient roughly around an axis in the IS direction. 


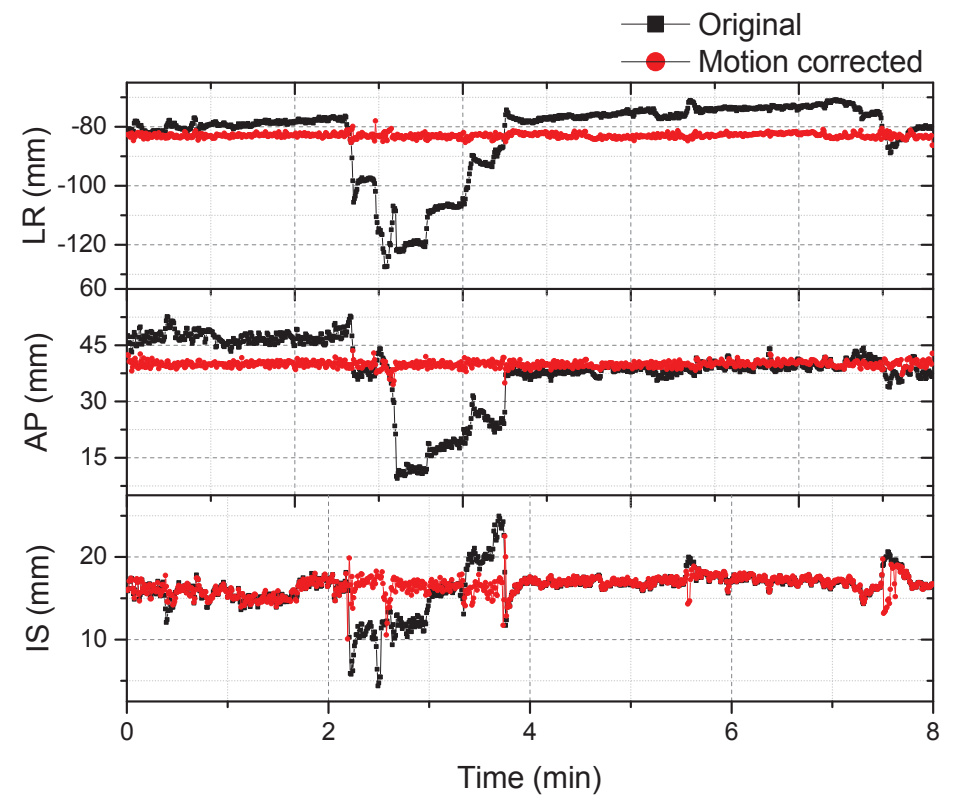

Figure 5.22: Non-MC and MC traces of the marker for Anon3512. 


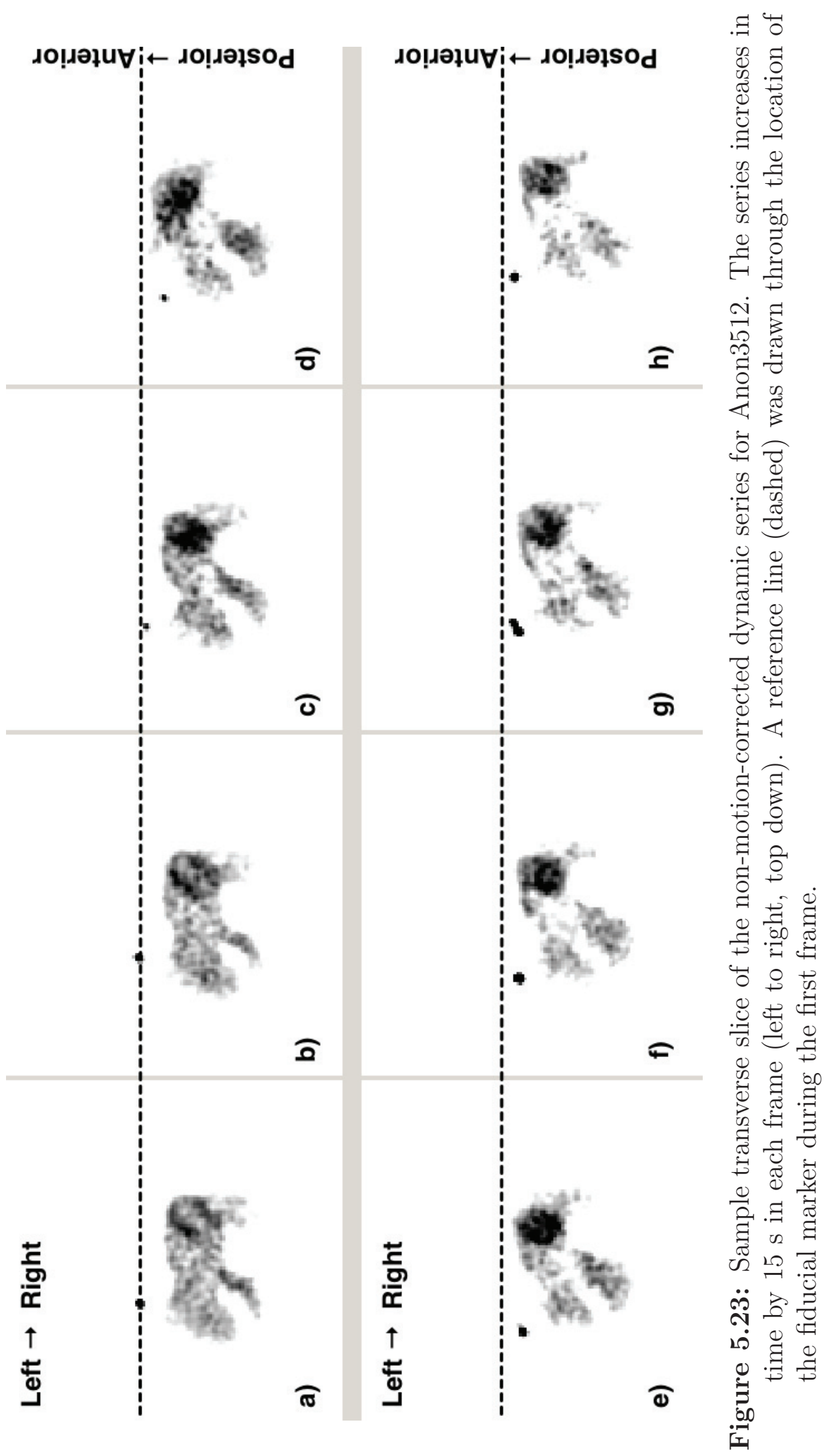




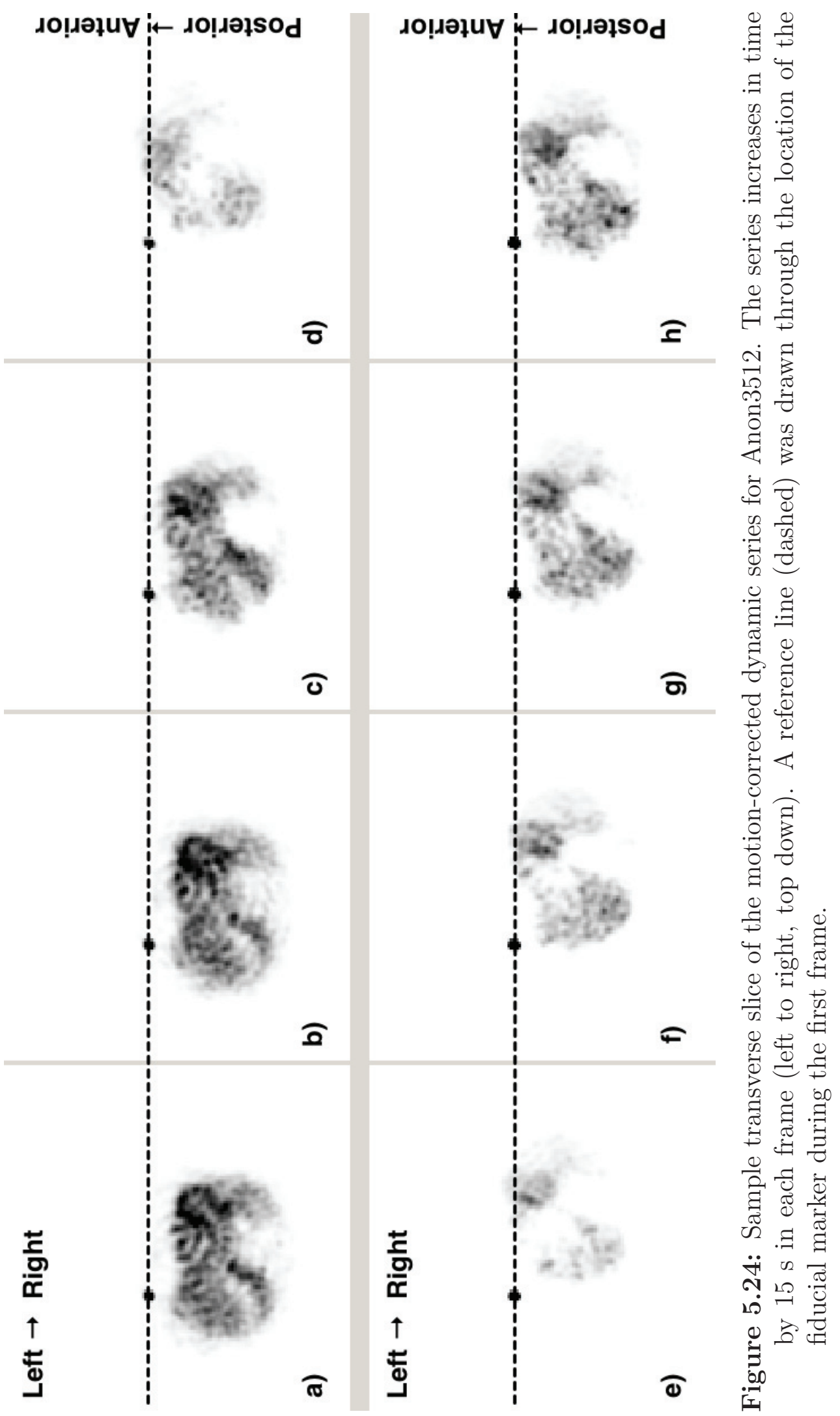




\subsubsection{Discussion}

It is feasible to use PeTrack motion traces for EBE motion correction in cardiac PET imaging. Results from the phantom studies show great promise at reducing patient body motion artefacts in PET imaging. Preliminary patient studies also demonstrate the potential for PeTrack in a clinical setting.

Several limitations remain in the present work. A single external fiducial marker is currently used to track motion. This limits the correction to $3 \mathrm{D}$ translations. As shown in Anon3512, patients may rotate their body during the scan. Such movements cannot be corrected with the current proposed method. This can be addressed by using additional markers to track motion. For example, if three markers are used, a rigid body transformation from the initial position of the markers to the final position can be calculated. [112]

The deformation of the heart during respiration is known to be non-rigid. [141] Current trends tend to use non-rigid image registration methods to account for this. $[124,142,143]$ The use of such motion correction techniques does not preclude using PeTrack to correct for gross patient body motion as a first step in the motion correction workflow. PeTrack has the distinct advantage of being able to react to inter-frame motion with temporal resolution on the order of $0.5 \mathrm{~s}$ or better, if using a higher activity marker.

PeTrack uses external fiducial markers placed on the abdomen to correct the motion of internal organs, such as the heart. The correlation between external abdominal motion and internal motion organ is largely variable between patients and even between studies of the same patient. $[44,80,125,126]$ This could introduce significant mismatches in the motion correction. Chan et al. [125] correlate an external 1D sur-

rogate motion signal and the $3 \mathrm{D}$ motion of the centroid of a target volume. This requires reconstructing multiple phase gated images and identifying the centroid of 
the target volume in each frame. Unfortunately, this technique is not directly applicable to the cardiac studies presented above. Rubidium- 82 has a very short half-life and redistributes itself rapidly through the heart. Thus the shape of the activity distribution changes rapidly through the scan, especially in the early frames when activity is high.

Figure 5.16 also illustrates another limit of the proposed motion correction method. The GE list mode format stores events into crystal index pairs. This discreteness limits the motion correction to the nearest crystal. This is illustrated in Figure 5.25. Events are associated to the nearest crystals. Thus, the magnitude of the motion vector in the IS direction has to be at least half a crystal pitch before the event is associated with the adjacent crystal. Subsequently, the event remains associated to the next crystal while the magnitude of the motion vector increases by one full crystal pitch. The issue is more complex in the transaxial plane (LR and AP directions) where the distance between parallel coincidence events decreases away from the central axis (see Figure 5.26). The discreteness of the motion correction can be seen in Figure 5.16 and Figure 5.17, where the LR and AP traces are mostly unchanged after motion correction. In this case the motion in those directions was always smaller than half the crystal pitch (i.e. $2.12 \mathrm{~mm}$ ). Possible workarounds involve weighted interpolation of the event between the crystals. $[144,145]$ However, this work was limited by the format of the GE list mode, which does not allow weighted events. The discreteness of the motion correction leads to a small residual motion of approximately one crystal pitch in size on the reconstructed images. This is on the order of the size of one voxel on the images $\left(3.91 \times 3.91 \times 3.27 \mathrm{~mm}^{3}\right)$.

The proposed motion correction scheme introduces a few issues. An event that is normally detected by the scanner may not be detected if the patient moves (e.g. if the event ends up outside the FOV). The reverse can also be true: an event that is not normally detected can move into the FOV. Various methods addressing those 


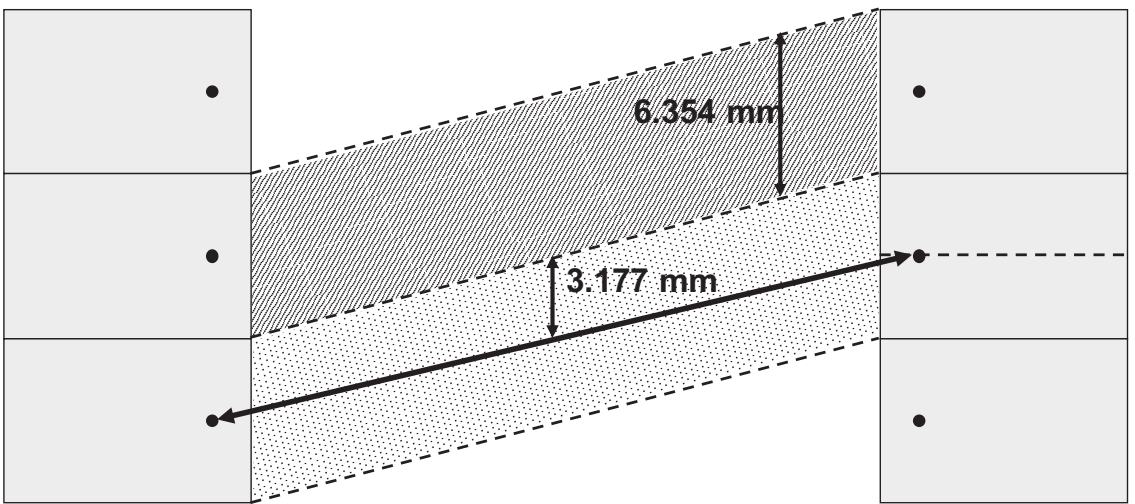

Centre of crystal at the average interaction depth

(a)

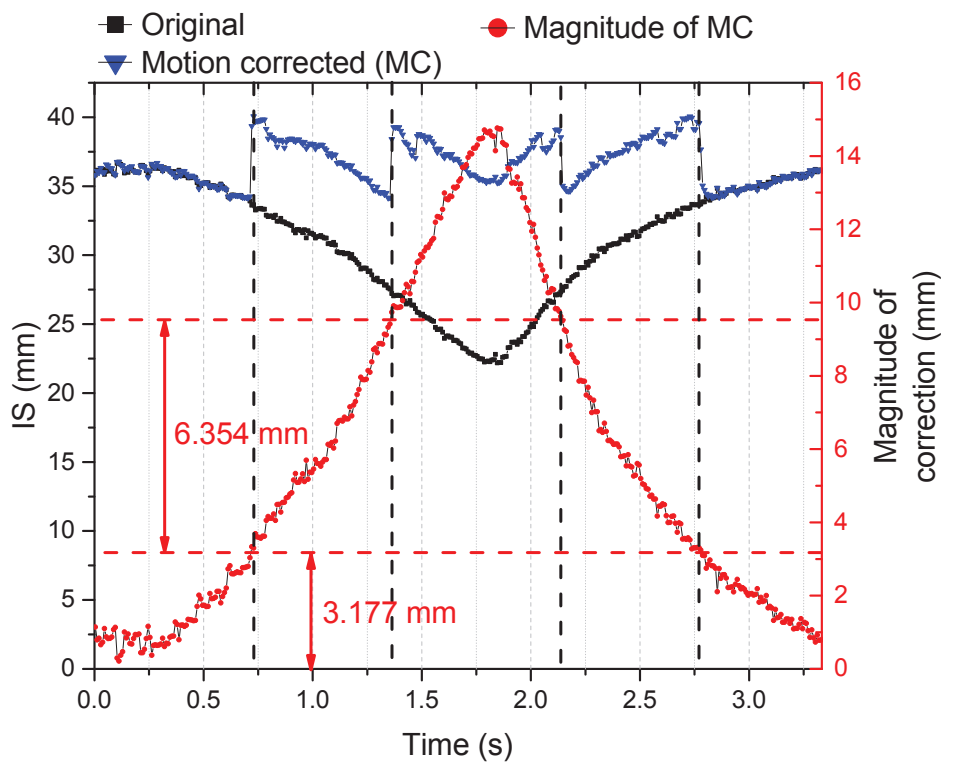

(b)

Figure 5.25: The motion correction is limited to correcting to the nearest crystal because the GE list mode format stores coincidence events as pairs of crystal indices. a) Events in the list mode are associated to the nearest crystal. An event has to move by half the crystal pitch before moving to the adjacent crystal pair. b) Close up of Figure 5.16. The discreteness of the motion correction causes discontinuities in the motion-corrected trace of the marker. 


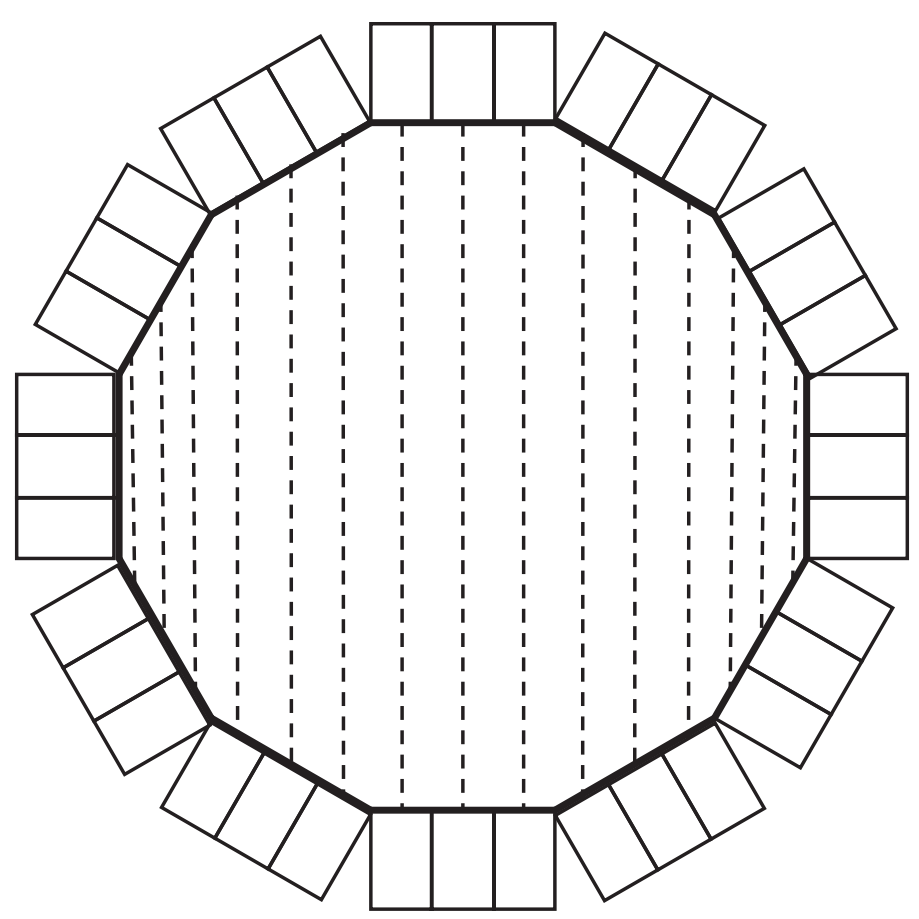

Figure 5.26: In the transaxial plane of the scanner, the distance between adjacent parallel coincidence events decreases away from the central axis.

issues have been proposed. [144, 146, 147] Those methods scale the sinograms with appropriate correction factors determined from the motion information. In addition, normalization corrections need to be applied to account for the differences in efficiency between the detector elements which detected the event and the crystals which would have detected the event if there had been no motion. ${ }^{8}$ Neglecting this effect leads to ring artefacts on the reconstructed image (see Figure 5.24). [144] When binning the events from list mode format to sinograms in the course of image reconstruction, proper normalization corrections should be applied.

The issues mentioned above can also be addressed by incorporating motion information directly in list mode expectation-maximization reconstruction algorithms (as opposed to reconstruction algorithms based on sinograms). Extensive work has been done on such algorithms and techniques. [116, 121,132]

\footnotetext{
${ }^{8}$ This efficiency includes both geometric factors and intrinsic crystal efficiency factors.
} 


\subsection{Conclusion}

This chapter has shown two potential applications of PeTrack motion tracking in PET imaging. The motion information can be applied to produce respiratory gated PET images. Phantom and patient studies demonstrate the effectiveness of PeTrackbased respiratory triggers. The PeTrack motion information can also be used for EBE motion correction of the raw list mode data. 


\section{Chapter 6}

\section{Conclusions and future work}

This thesis presented potential applications of PeTrack, a 3D tracking system which uses low activity fiducial positron emission markers. Chapter 1 motivated the need for motion tracking and compensation in radiation therapy and in PET imaging. Patient body motion during those medical procedures can lead to significant errors. Motion detection and compensation are required to address this issue. Chapter 2 gave an overview of basic positron emission physics and of PeTrack. The multimarker expectation-maximization clustering algorithm, the automatic initialization of the algorithm, and the fail-safe methods were described. The performance evaluation of PeTrack was also reported.

Chapter 3 covered the application of PeTrack for respiratory gated radiation therapy. The dose delivered to the PeTrack detectors by scatter from the radiation therapy

beams was calculated by Monte Carlo simulations. It was found that this dose will be negligible in a clinical setting. The feasibility of respiratory gated radiotherapy using PeTrack was assessed by measuring dose distributions on radiochromic films during gated and non-gated beam deliveries. An anthropomorphic dynamic thorax phantom was used with real patient breathing motion patterns. Beam delivery was done with an Elekta Precise linac. This phantom study demonstrated that the blurring of the 
dose distribution is greatly reduced on the gated deliveries compared to the non-gated deliveries.

Chapter 4 explored the use of PeTrack for motion tracking of an external fiducial marker during PET imaging. The very low signal of the fiducial marker compared to the physiological tracer activity within the patient makes tracking the motion very challenging. Modifications to the PeTrack algorithm in the form of new background rejection steps can dramatically increase the SNR and allow tracking of the marker. Monte Carlo and phantom studies with an anthropomorphic thorax phantom for cardiac PET imaging confirmed that PeTrack can provide motion tracking during PET imaging. The accuracy and precision of the algorithm remain in the sub-millimetre range.

Chapter 5 presented two proof of concept applications of PeTrack for motion compensated PET imaging. First, motion traces from PeTrack were used to generate respiratory triggers for phase based gating. The triggers were compared to the triggers produced by the Varian RPM system for the same patient studies. The number and distribution of respiratory triggers were very similar. Visual assessment of the gated PET images showed that PeTrack gating produces images similar to the RPM gating. In some cases, PeTrack gating captures more of the heart motion than the RPM gating. Second, the feasibility of motion correction at the list mode level was evaluated. The list mode data from phantom and patient studies were corrected event-by-event by translating each coincidence event to a reference position. The motion corrected static and dynamic reconstructed images showed great improvement over the original non-motion corrected data. A particular patient study, however, demonstrated the limitations of the current implementation of the motion correction: only translational motion can be corrected if a single fiducial marker was tracked and the list mode format limits the magnitude of the correction vector to the size of a detector element. 
PeTrack presents several advantages over other available motion compensation techniques. In the context of respiratory-gated radiotherapy, PeTrack can track the 3D internal tumour motion directly with the use of implanted fiducial markers. The dose delivered to the patient by the positron-emitting markers is better confined to the target volume than the dose from $\mathrm{x}$ ray tracking of internal markers and can potentially be taken into account during treatment planning. In the context of motion-compensated PET imaging, PeTrack is a software solution which requires no additional costly hardware with the exception of the fiducial markers. The low activity of the markers will deposit a negligible dose to the patient compared to the dose delivered by the radiotracer. The signal from the PeTrack markers is recorded directly in the raw list mode data which makes it available for retrospective studies as long as the list mode data are preserved. This also means that the PeTrack motion information is intrinsically synchronized with the PET data. No spatial coregistration is required between PeTrack and the PET scanner. Patient setup is also fast and straightforward. The PeTrack marker simply needs to be secured to the abdomen of the patient. However, staff should ensure that the marker is visible within the FOV of the scanner, which can be confirmed on the setup CT. Those advantages and the performance of the system should make PeTrack a desirable solution for cancer and imaging clinics.

\subsection{Future work}

Many future research avenues can be pursued. In the case of respiratory gated radiotherapy, PeTrack should be tested on a clinical linac. The beam on and off delays should be fine tuned and brought to reasonable levels $(<0.2 \mathrm{~s})$. As outlined in Section 3.3.4, this should be easily achievable clinically on Elekta linacs with a fast 
tuning magnetron with the use of the Elekta Response gating interface. Theoretically, it should also be possible to integrate PeTrack with other brands of linacs, as long as the time delays can be kept reasonably short and a way to interrupt and resume the beam delivery is available. Clinically relevant dosimetry analysis could be done on radiochromic films with the delivery of a sample treatment plan. Full 3D motion of the phantom could also be added for more complexity. Beam repositioning for real time tumour tracking using PeTrack could also be explored. For example, PeTrack could be used in intensity modulated radiotherapy (IMRT) delivered with a dynamic multi leaf collimator (MLC). MLCs are made of individual highly attenuating sheets ("leaves") that can dynamically move in and out of the path of the beam during treatment, allowing the beam to conform to various shapes. The PeTrack motion information could be fed to the linac during IMRT to dynamically re-arrange the leaves of the MLC. This would allow the beam to follow the motion of the target volume in real time. Dynamic MLC tumour tracking has been proposed before, but the first treatment of prostate cancer patients using electromagnetic-guided MLC tracking was only reported recently. [148,149] PeTrack could provide the real-time tumour tracking necessary for MLC tracking treatments. Since the majority of commercially available linacs are equipped with an MLC, this could broaden the applicability of PeTrack-guided MLC tracking.

In PET imaging, several options are available. The literature suggests amplitudebased methods perform better than phase-based ones. Therefore amplitude-based gating should be investigated and compared to the RPM-based gating. Two additional fiducial markers could be placed on the abdomen of patients for six degrees of freedom rigid body motion correction. This could potentially offer a way to correct the rotational motion observed in one of the patient studies. Also, proper normalization of the motion corrected events should be done, as proposed by various 
methods. [144, 146, 147] This would allow more clinically relevant quantitative comparisons of the motion corrected images and would prevent some of the ring artefacts observed on them. The PeTrack algorithm could potentially be modified to directly track the internal motion of the walls of the heart from the list mode data. This would remove the need for external markers, making PeTrack fully software-based and even simpler to apply clinically. However, the walls of the heart may prove to be particularly difficult to track in ${ }^{82} \mathrm{Rb}$ cardiac PET imaging due to rapidly changing activity distribution as the blood flows through the heart. To that end, analyzing the spatial distribution of the LORs recorded during cardiac PET imaging might yield useful information. 


\section{References}

[1] M. Chamberland, R. Wassenaar, B. Spencer, and T. Xu. "Performance evaluation of real-time motion tracking using positron emission fiducial markers." Medical Physics 38(2), 810-819 (2011).

[2] M. Chamberland. Performance evaluation and algorithm development for realtime tumour tracking using positron emission markers. Master's thesis, Carleton University, Ottawa (ON) (2009).

[3] M. Soleimani. Detector development for positron emission based real-time tumor tracking. Master's thesis, Carleton University, Ottawa (ON) (2009).

[4] J. W. Choi, J. B. Seo, K.-H. Do, S. I. Choi, W. Lee, S. M. Ko, S. H. Lee, J. S. Lee, J.-W. Song, K.-S. Song, and T.-H. Lim. "Comparison of transaxial source images and 3-plane, thin-slab maximal intensity projection images for the diagnosis of coronary artery stenosis using ECG-gated cardiac CT." Korean Journal of Radiology 7(1), 20-27 (2006).

[5] Canadian Cancer Society's Advisory Committee on Cancer Statistics. Canadian Cancer Statistics 2014. Canadian Cancer Society, Toronto, ON (2014).

[6] C. de Oliveira, K. E. Bremner, R. Pataky, N. Gunraj, M. Haq, K. Chan, W. Y. Cheung, J. S. Hoch, S. Peacock, and M. D. Krahn. "Trends in use and cost of initial cancer treatment in Ontario: a population-based descriptive study." CMAJ Open 1(4), E151-E158 (2013).

[7] M. van Herk. "Errors and margins in radiotherapy." Seminars in Radiation Oncology 14(1), 52-64 (2004).

[8] I. Jacobs, J. Vanregemorter, and P. Scalliet. "Influence of respiration on calculation and delivery of the prescribed dose in external radiotherapy." Radiotherapy and Oncology 39(2), 123-128 (1996). 
[9] E. Barnes, B. Murray, D. Robinson, L. Underwood, J. Hanson, and W. Roa. "Dosimetric evaluation of lung tumor immobilization using breath hold at deep inspiration." International Journal of Radiation Oncology, Biology, Physics 50(4), 1091-1098 (2001).

[10] S. Erridge, Y. Seppenwoolde, S. Muller, M. van Herk, K. de Jaeger, J. Belderbos, L. Boersma, and J. V. Lebesque. "Portal imaging to assess set-up errors, tumor motion and tumor shrinkage during conformal radiotherapy of non-small cell lung cancer." Radiotherapy and Oncology 66(1), 75-85 (2003).

[11] I. Grills, D. Yan, A. Martinez, F. Vicini, J. Wong, and L. Kestin. "Potential for reduced toxicity and dose escalation in the treatment of inoperable non-small-cell lung cancer: A comparison of intensity-modulated radiation therapy (IMRT), 3D conformal radiation, and elective nodal irradiation." International Journal of Radiation Oncology, Biology, Physics 57(3), 875-890 (2003).

[12] H. Wu, G. Sharp, Q. Zhao, H. Shirato, and S. Jiang. "Statistical analysis and correlation discovery of tumor respiratory motion." Physics in Medicine and Biology 52, 4761-4774 (2007).

[13] M. Engelsmann, E. Damen, K. De Jaeger, K. M. van Ingen, and B. J. Mijnheer. "The effect of breathing and set-up errors on the cumulative dose to a lung tumor." Radiotherapy and Oncology 60(1), 95-105 (2001).

[14] P. Keall, G. S. Mageras, J. Balter, R. Emery, K. Forster, S. Jiang, J. Kapatoes, D. Low, M. Murphy, B. Murray, C. R. Ramsey, M. van Herk, S. S. Vedam, J. Wong, and E. Yorke. "The management of respiratory motion in radiation oncology." Medical Physics 33(10), 3874-3900 (2006).

[15] G. G. Zhang, H.-H. M. Yu, C. W. Stevens, T. J. Dilling, S. E. Hoffe, E. G. Moros, and V. Feygelman. "Motion management in stereotactic body radiotherapy." Journal of Nuclear Medicine 65 Radiation Therapy S6(012) (2013).

[16] E. M. Rohren, T. G. Turkington, and R. E. Coleman. "Clinical applications of PET in oncology." Radiology 231(2), 305-332 (2004).

[17] R. Nakazato, D. S. Berman, E. Alexanderson, and P. Slomka. "Myocardial perfusion imaging with PET." Imaging in Medicine 5(1), 35-46 (2013).

[18] P. G. Camici and O. E. Rimoldi. "The clinical value of myocardial blood flow measurement." Journal of Nuclear Medicine 50(7), 1076-1087 (2009). 
[19] F. J. Klocke and D. C. Lee. "Absolute myocardial blood flow." The Journal of the American College of Cardiology: Cardiovascular Imaging: Cardiovascular Imaging 4(9), 999-1001 (2011).

[20] T. H. Schindler, H. R. Schelbert, A. Quercioli, and V. Dilsizian. "Cardiac PET imaging for the detection and monitoring of coronary artery disease and microvascular health." The Journal of the American College of Cardiology: Cardiovascular Imaging: Cardiovascular Imaging 3(6), 623-640 (2010).

[21] S. Dai, C. Bancej, and A. Bienek. "Tracking heart disease and stroke in Canada 2009." Chronic Diseases in Canada 29(4), 192-195 (2009).

[22] J. Dinges, S. G. Nekolla, and R. A. Bundschuh. "Motion artifacts in oncological and cardiac PET imaging." PET Clinics 8(1), 1-9 (2013).

[23] R. Klein, C. Hunter, R. Beanlands, and R. deKemp. "Prevalence of patient motion in dynamic PET." The Journal of Nuclear Medicine 52(Supplement 1), 2105-2105 (2011).

[24] V. Bettinardi, E. De Bernardi, L. Presotto, and M. C. Gilardi. "Motion-tracking hardware and advanced applications in PET and PET/CT." PET Clinics 8(1), $11-28$ (2013).

[25] T. Xu, J. Wong, P. Shikhaliev, J. Ducote, M. Al-Ghazi, and S. Molloi. "Realtime tumor tracking using implanted positron emission markers: Concept and simulation study." Medical Physics 33(7), 2598-2609 (2006).

[26] E. B. Podgoršak. Radiation physics for medical physicists. Springer, Berlin, 2nd edition (2010).

[27] P. Zanzonico. "Positron emission tomography: a review of basic principles, scanner design and performance, and current systems." Seminars in Nuclear Medicine 34(2), 87-111 (2004).

[28] D. K. Shibuya, E. Yoshida, F. Nishikido, T. Suzuki, T. Tsuda, N. Inadama, T. Yamaya, and H. Murayama. "Limit of spatial resolution in FDG-PET due to annihilation photon non-collinearity." In "World Congress on Medical Physics and Biomedical Engineering 2006," pages 1667-1671. Springer Berlin Heidelberg (2007).

[29] M. Conti. "Focus on time-of-flight PET: the benefits of improved time resolution." European Journal of Nuclear Medicine and Molecular Imaging 38(6), 1147-1157 (2011). 
[30] S. E. Derenzo, W.-S. Choong, and W. W. Moses. "Fundamental limits of scintillation detector timing precision." Physics in Medicine and Biology 59(13), 3261-3286 (2014).

[31] S. Surti. "Update on Time-of-Flight PET Imaging." Journal of Nuclear Medicine 56(1), 98-105 (2015).

[32] S. Razavi. Sensitivity and distortion studies of positron emission tracking system (PeTrack). Master's thesis, Carleton University, Ottawa (ON) (2014).

[33] O. Gundogdu. "Positron emission tomography particle tracking using cluster analysis." Nuclear Instruments and Methods In Physics Research Section A 534(3), 562-576 (2004).

[34] D. Litzenberg, J. Moran, and B. Fraass. "Incorporation of realistic delivery limitations into dynamic MLC treatment delivery." Medical Physics 29(5), 810-820 (2002).

[35] M. F. Fast, S. Nill, J. L. Bedford, and U. Oelfke. "Dynamic tumor tracking using the Elekta Agility MLC." Medical Physics 41(11), 111719 (5pp.) (2014).

[36] Y. Seppenwoolde, H. Shirato, K. Kitamura, S. Shimizu, M. van Herk, J. V. Lebesque, and K. Miyasaka. "Precise and real-time measurement of 3D tumor motion in lung due to breathing and heartbeat, measured during radiotherapy." International Journal of Radiation Oncology, Biology, Physics 53(4), 822-834 (2002).

[37] Y. Seppenwoolde, R. I. Berbeco, S. Nishioka, H. Shirato, and B. Heijmen. "Accuracy of tumor motion compensation algorithm from a robotic respiratory tracking system: A simulation study." Medical Physics 34(7), 2774-2784 (2007).

[38] G. Sharp, S. Jiang, S. Shimizu, and H. Shirato. "Prediction of respiratory tumour motion for real-time image-guided radiotherapy." Physics in Medicine and Biology 49(3), 425-440 (2004).

[39] H. D. Kubo and B. C. Hill. "Respiration gated radiotherapy treatment: a technical study." Physics in Medicine and Biology 41(1), 83-91 (1996).

[40] T. Zhang, H. Keller, M. J. O’Brien, T. R. Mackie, and B. Paliwal. "Application of the spirometer in respiratory gated radiotherapy." Medical Physics 30(12), 3165-3171 (2003). 
[41] X. A. Li, C. C. Stepaniak, and E. E. Gore. "Technical and dosimetric aspects of respiratory gating using a pressure-sensor motion monitoring system." Medical Physics 33(1), 145-154 (2006).

[42] Y. Otani, I. Fukuda, N. Tsukamoto, Y. Kumazaki, H. Sekine, E. Imabayashi, O. Kawaguchi, T. Nose, T. Teshima, and T. Dokiya. "A comparison of the respiratory signals acquired by different respiratory monitoring systems used in respiratory gated radiotherapy." Medical Physics 37(12), 6178-6186 (2010).

[43] S. S. Vedam, P. J. Keall, V. R. Kini, H. Mostafavi, H. P. Shukla, and R. Mohan. "Acquiring a four-dimensional computed tomography dataset using an external respiratory signal." Physics in Medicine and Biology 48(1), 45-62 (2003).

[44] J. Hoisak, K. Sixel, R. Tirona, and P. Cheung. "Correlation of lung tumor motion with external surrogate indicators of respiration." International Journal of Radiation Oncology, Biology, Physics 60(4), 1298-1306 (2004).

[45] Z. Chang, T. Liu, J. Cai, Q. Chen, Z. Wang, and F.-F. Yin. "Evaluation of integrated respiratory gating systems on a Novalis Tx system." Journal of Applied Clinical Medical Physics 12(3), 71-79 (2011).

[46] C. Nelson, G. Starkschall, P. Balter, M. J. Fitzpatrick, J. A. Antolak, N. Tolani, and K. Prado. "Respiration-correlated treatment delivery using feedback-guided breath hold: A technical study." Medical Physics 32(1), 175-181 (2005).

[47] N. Koch, H. H. Liu, G. Starkschall, M. Jacobson, K. Forster, Z. Liao, R. Komaki, and C. W. Stevens. "Evaluation of internal lung motion for respiratory-gated radiotherapy using MRI: Part I-correlating internal lung motion with skin fiducial motion." International Journal of Radiation Oncology, Biology, Physics 60(5), 1459-1472 (2004).

[48] D. Ionascu, S. B. Jiang, S. Nishioka, H. Shirato, and R. I. Berbeco. "Internalexternal correlation investigations of respiratory induced motion of lung tumors." Medical Physics 34(10), 3893-3903 (2007).

[49] H. Fayad, T. Pan, J. F. Clement, and D. Visvikis. "Technical note: Correlation of respiratory motion between external patient surface and internal anatomical landmarks." Medical Physics 38(6), 3157-3164 (2011).

[50] S. Ahn, B. Yi, Y. Suh, J. Kim, S. Lee, S. Shin, and E. Choi. "A feasibility study on the prediction of tumour location in the lung from skin motion." The British Journal of Radiology 77(919), 588-596 (2014). 
[51] F. Ernst, R. Bruder, A. Schlaefer, and A. Schweikard. "Correlation between external and internal respiratory motion: a validation study." International Journal of Computer Assisted Radiology and Surgery 7(3), 483-492 (2012).

[52] H. Shirato, S. Shimizu, T. Kunieda, K. Kitamura, M. van Herk, K. K. Kagei, T. Nishioka, S. Hashimoto, K. Fujita, H. Aoyama, K. Tsuchiya, K. Kudo, and K. Miyasaka. "Physical aspects of a real-time tumor-tracking system for gated radiotherapy." International Journal of Radiation Oncology, Biology, Physics 48(4), 1187-1195 (2000).

[53] H. Shirato, M. Oita, K. Fujita, Y. Watanabe, and K. Miyasaka. "Feasibility of synchronization of real-time tumor-tracking radiotherapy and intensitymodulated radiotherapy from viewpoint of excessive dose from fluoroscopy." International Journal of Radiation Oncology, Biology, Physics 60(1), 335-341 (2004).

[54] X. Tang, G. Sharp, and S. Jiang. "Fluoroscopic tracking of multiple implanted fiducial markers using multiple object tracking." Physics in Medicine and Biology 52, 4081-4098 (2007).

[55] J.-Y. Jin, F.-F. Yin, S. E. Tenn, P. M. Medin, and T. D. Solberg. "Use of the BrainLAB ExacTrac X-Ray 6D system in image-guided radiotherapy." Medical Dosimetry 33(2), 124-134 (2008).

[56] M. Hoogeman, J.-B. Prévost, J. Nuyttens, J. Pöll, P. Levendag, and B. Heijmen. "Clinical accuracy of the respiratory tumor tracking system of the CyberKnife: Assessment by analysis of log files." International Journal of Radiation Oncology, Biology, Physics 74(1), 297-303 (2009).

[57] K. Takayama, T. Mizowaki, M. Kokubo, N. Kawada, H. Nakayama, Y. Narita, K. Nagano, Y. Kamino, and M. Hiraoka. "Initial validations for pursuing irradiation using a gimbals tracking system." Radiotherapy and Oncology 93(1), 45-49 (2009).

[58] M. Hiraoka, Y. Matsuo, A. Sawada, N. Ueki, Y. Miyaba, M. Nakamura, S. Yano, S. Kaneko, T. Mizowaki, and M. Kokubo. "Realization of dynamic tumor tracking irradiation with real-time monitoring in lung tumor patients using a gimbaled x-ray head radiation therapy equipment." International Journal of Radiation Oncology, Biology, Physics 84(3), S560-S561 (2012).

[59] T. Ono, Y. Miyabe, M. Yamada, T. Shiinoki, A. Sawada, S. Kaneko, H. Monzen, T. Mizowaki, M. Kokubo, and M. Hiraoka. "Geometric and dosimetric accuracy 
of dynamic tumor-tracking conformal arc irradiation with a gimbaled x-ray head." Medical Physics 41(3), 031705 (8pp.) (2014).

[60] C. Silva, D. Mateus, M. Eiras, and S. Vieira. "Calypso 4D localization system: A review." Journal of Radiotherapy in Practice 13(04), 473-483 (2014).

[61] T. Willoughby, P. Kupelian, J. Pouliot, and K. Shinohara. "Target localization and real-time tracking using the Calypso 4D localization system in patients with localized prostate cancer." International Journal of Radiation Oncology, Biology, Physics 65(2), 528-534 (2006).

[62] A. P. Shah, P. A. Kupelian, T. R. Willoughby, and S. L. Meeks. "Expanding the use of real-time electromagnetic tracking in radiation oncology." Journal of Applied Clinical Medical Physics 12(4), 34-49 (2011).

[63] J. Kindblom, A.-M. Ekelund-Olvenmark, and H. Syren. "High precision transponder localization using a novel electromagnetic positioning system in patients with localized prostate cancer." Radiotherapy and Oncology 90(3), 307-311 (2009).

[64] T. Shchory, D. Schifter, R. Lichtman, D. Neustadter, and B. W. Corn. "Tracking accuracy of a real-time fiducial tracking system for patient positioning and monitoring in radiation therapy." International Journal of Radiation Oncology, Biology, Physics 78(4), 1227-1234 (2010).

[65] Q. Q. Fan, A. A. Nanduri, S. S. Mazin, and L. L. Zhu. "Emission guided radiation therapy for lung and prostate cancers: A feasibility study on a digital patient." Medical Physics 39(11), 7140-7152 (2012).

[66] Q. Fan, A. Nanduri, J. Yang, T. Yamamoto, B. Loo, E. Graves, L. Zhu, and S. Mazin. "Toward a planning scheme for emission guided radiation therapy (EGRT): FDG based tumor tracking in a metastatic breast cancer patient." Medical Physics 40(8), 081708 (12pp.) (2013).

[67] I. Kawrakow, E. Mainegra-Hing, D. Rogers, F. Tessier, and B. R. Walters. "The EGSnrc code system: Monte Carlo simulation of electron and photon transport ." Technical report, NRCC Report PIRS-701, Ottawa, Canada (2000).

[68] I. Kawrakow, E. Mainegra-Hing, F. Tessier, and B. R. Walters. "The EGSnrc C++ class library." Technical report, NRC Report PIRS-898 (rev A), Ottawa, Canada (2009). 
[69] R. Mohan. "Energy and angular distributions of photons from medical linear accelerators." Medical Physics 12(5), 592-597 (1985).

[70] I. J. Chetty, B. Curran, J. E. Cygler, J. J. DeMarco, G. Ezzell, B. A. Faddegon, I. Kawrakow, P. J. Keall, H. Liu, C. M. C. Ma, D. W. O. Rogers, J. Seuntjens, D. Sheikh-Bagheri, and J. V. Siebers. "Report of the AAPM Task Group No. 105: Issues associated with clinical implementation of Monte Carlo-based photon and electron external beam treatment planning." Medical Physics 34(12), 4818-4853 (2007).

[71] J. Ramos-Méndez, J. Perl, B. Faddegon, J. Schümann, and H. Paganetti. "Geometrical splitting technique to improve the computational efficiency in Monte Carlo calculations for proton therapy." Medical Physics 40(4), 041718 (10pp.) (2013).

[72] I. Kawrakow, D. W. O. Rogers, and B. R. B. Walters. "Large efficiency improvements in BEAMnrc using directional bremsstrahlung splitting." Medical Physics 31(10), 2883-2898 (2004).

[73] M. Fragoso, I. Kawrakow, B. A. Faddegon, T. D. Solberg, and I. J. Chetty. "Fast, accurate photon beam accelerator modeling using BEAMnrc: A systematic investigation of efficiency enhancing methods and cross-section data." Medical Physics 36(12), 5451-5466 (2009).

[74] M. Treutwein, M. Hipp, O. Koelbl, and L. Bogner. "IMRT of prostate cancer ." Strahlentherapie Und Onkologie 185(6), 379-383 (2009).

[75] D. Gasic, L. Ohlhues, N. P. Brodin, L. S. Fog, T. Pommer, J. P. Bangsgaard, and P. M. A. Rosenschöld. "A treatment planning and delivery comparison of volumetric modulated arc therapy with or without flattening filter for gliomas, brain metastases, prostate, head/neck and early stage lung cancer." Acta Oncologica 53(8), 1005-1011 (2014).

[76] K. R. Voong, K. McSpadden, C. C. Pinnix, F. Shihadeh, V. Reed, M. R. Salehpour, I. Arzu, H. Wang, D. Hodgson, J. Garcia, M. Aristophanous, and B. S. Dabaja. "Dosimetric advantages of a "butterfly" technique for intensity-modulated radiation therapy for young female patients with mediastinal Hodgkin's lymphoma." Radiation Oncology 9(94) (2014).

[77] A. E. Lujan, E. W. Larsen, J. Balter, and R. K. T. Haken. "A method for incorporating organ motion due to breathing into 3D dose calculations." Medical Physics 26(5), 715-720 (1999). 
[78] S. A. Nehmeh, Y. E. Erdi, T. Pan, and E. Yorke. "Quantitation of respiratory motion during 4D-PET/CT acquisition." Medical Physics 31(6), 1333-1338 (2004).

[79] J. M. Blackall, S. Ahmad, M. E. Miquel, J. R. McClelland, D. B. Landau, and D. J. Hawkes. "MRI-based measurements of respiratory motion variability and assessment of imaging strategies for radiotherapy planning." Physics in Medicine and Biology 51, 4147-4169 (2006).

[80] D. Ionascu, S. Jiang, S. Nishioka, H. Shirato, and R. I. Berbeco. "Internalexternal correlation investigations of respiratory induced motion of lung tumors." Medical Physics 34(10), 3893-3903 (2007).

[81] J. Cai, P. W. Read, and K. Sheng. "The effect of respiratory motion variability and tumor size on the accuracy of average intensity projection from four-dimensional computed tomography: An investigation based on dynamic MRI." Medical Physics 35(11), 4974-4981 (2008).

[82] A. Cherpak, W. Ding, A. Hallil, and J. Cygler. "Evaluation of a novel 4D in vivo dosimetry system." Medical Physics 36(5), 1672-1679 (2009).

[83] Q.-S. Chen, M. Weinhous, F. C. Deibel, and J. Ciezki. "Fluoroscopic study of tumor motion due to breathing: facilitating precise radiation therapy for lung cancer patients." Medical Physics 28(9), 1850-1856 (2001).

[84] G. Cui, D. J. Housley, F. Chen, V. K. Mehta, and D. M. Shepard. "Delivery efficiency of an Elekta linac under gated operation." Journal of Applied Clinical Medical Physics 15(5), 2-11 (2014).

[85] P. M. Evans, J. R. N. Symonds-Tayler, R. Colgan, G. D. Hugo, N. Letts, and C. Sandin. "Gating characteristics of an Elekta radiotherapy treatment unit measured with three types of detector." Physics in Medicine and Biology 55(8), N201-N210 (2010).

[86] P. Freislederer, M. Reiner, W. Hoischen, A. Quanz, C. Heinz, F. Walter, C. Belka, and M. Soehn. "Characteristics of gated treatment using an optical surface imaging and gating system on an Elekta linac." Radiation Oncology 10(68) (2015).

[87] G. J. Budgell, C. Martens, and F. Claus. "Improved delivery efficiency for step and shoot intensity modulated radiotherapy using a fast-tuning magnetron." Physics in Medicine and Biology 46(11), N253-N261 (2001). 
[88] P. Kozma and P. Kozma Jr. "Radiation resistivity of BGO crystals due to lowenergy gamma-rays." Nuclear Instruments and Methods In Physics Research Section A 501, 499-504 (2003).

[89] K. Weibert, S. Biller, T. G. Wendt, and T. Wiezorek. "Dosimetry of a linear accelerator under respiratory gating." Zeitschrift Fur Medizinische Physik 19(2), 136-141 (2009).

[90] W. L. Smith and N. Becker. "Time delays and margins in gated radiotherapy." Journal of Applied Clinical Medical Physics 10(3), 140-154 (2009).

[91] M. van Herk, P. Remeijer, C. Rasch, and J. V. Lebesque. "The probability of correct target dosage: dose-population histograms for deriving treatment margins in radiotherapy." International Journal of Radiation Oncology, Biology, Physics 47(4), 1121-1135 (2000).

[92] H. Shirato, K. Suzuki, G. Sharp, K. Fujita, and R. Onimaru. "Speed and amplitude of lung tumor motion precisely detected in four-dimensional setup and in real-time tumor-tracking radiotherapy." International Journal of Radiation Oncology, Biology, Physics 64(4), 1229-1236 (2006).

[93] D. Visvikis, O. Barret, T. Fryer, A. Turzo, F. Lamare, C. Cheze le Rest, and Y. Bizais. "A posteriori respiratory motion gating of dynamic PET images." In "IEEE Nuclear Science Symposium Conference Record," pages 3276-3280. IEEE (2003).

[94] P. J. Schleyer, M. J. O’Doherty, S. F. Barrington, and P. K. Marsden. "Retrospective data-driven respiratory gating for PET/CT." Physics in Medicine and Biology 54(7), 1935-1950 (2009).

[95] G. J. Klein, B. W. Reutter, E. H. Botvinick, T. F. Budinger, and R. H. Huesman. "Fine-scale motion detection using intrinsic list mode PET information." In "Proceedings IEEE Workshop on Mathematical Methods in Biomedical Image Analysis (MMBIA 2001)," pages 71-78. IEEE Comput. Soc (2001).

[96] R. A. Bundschuh, A. Martinez-Moeller, M. Essler, M.-J. Martinez, S. G. Nekolla, S. I. Ziegler, and M. Schwaiger. "Postacquisition detection of tumor motion in the lung and upper abdomen using list-mode PET data: A feasibility study." Journal of Nuclear Medicine 48(5), 758-763 (2007).

[97] F. Buther, M. Dawood, L. Stegger, F. Wubbeling, M. Schafers, O. Schober, and K. P. Schafers. "List Mode-Driven Cardiac and Respiratory Gating in PET." Journal of Nuclear Medicine 50(5), 674-681 (2009). 
[98] S. A. Nehmeh, Y. E. Erdi, K. E. Rosenzweig, H. Schoder, S. M. Larson, O. D. Squire, and J. L. Humm. "Reduction of respiratory motion artifacts in PET imaging of lung cancer by respiratory correlated dynamic PET: methodology and comparison with respiratory gated PET." Journal of Nuclear Medicine 44(10), 1644-1648 (2003).

[99] B. Nazarparvar, M. Shamsaei, and H. Rajabi. "Correction of head movements in positron emission tomography using point source tracking system: a simulation study." Annals of Internal Medicine 26(1), 7-15 (2012).

[100] F. Büther, I. Ernst, J. Hamill, H. T. Eich, O. Schober, M. Schaefers, and K. P. Schaefers. "External radioactive markers for PET data-driven respiratory gating in positron emission tomography." European Journal of Nuclear Medicine and Molecular Imaging 40(4), 602-614 (2013).

[101] P. J. Noonan, J. Howard, D. Tout, I. Armstrong, H. A. Williams, T. F. Cootes, W. A. Hallett, and R. Hinz. "Accurate markerless respiratory tracking for gated whole body PET using the Microsoft Kinect." In "IEEE Nuclear Science Symposium and Medical Imaging Conference," pages 3973-3974. IEEE (2012).

[102] M. R. Palmer and G. L. Brownell. "Annihilation density distribution calculations for medically important positron emitters." IEEE Transactions on Medical Imaging 11(3), 373-378 (1992).

[103] M. Palmer, X. Zhu, and J. Parker. "Modeling and simulation of positron range effects for high resolution PET imaging." IEEE Transactions on Nuclear Science 52(5), 1391-1395 (2005).

[104] S. E. Derenzo. "Precision measurement of annihilation point spread distributions for medically important positron emitters." In "5th International Conference on Positron Annihilation," Lake Yamanaka, Japan (1979).

[105] V. Bettinardi, L. Presotto, E. Rapisarda, M. Picchio, L. Gianolli, and M. C. Gilardi. "Physical Performance of the new hybrid PET/CT Discovery-690." Medical Physics 38(10), 5394-5411 (2011).

[106] S. Jan, D. Benoit, E. Becheva, T. Carlier, F. Cassol, P. Descourt, T. Frisson, L. Grevillot, L. Guigues, L. Maigne, C. Morel, Y. Perrot, N. Rehfeld, D. Sarrut, D. R. Schaart, S. Stute, U. Pietrzyk, D. Visvikis, N. Zahra, and I. Buvat. "GATE V6: A major enhancement of the GATE simulation platform enabling modelling of CT and radiotherapy." Physics in Medicine and Biology 56, 881901 (2011). 
[107] C. C. Watson. "New, faster, image-based scatter correction for 3D PET." IEEE Transactions on Nuclear Science 47(4), 1587-1594 (2000).

[108] J. M. Balter, K. L. Lam, C. J. McGinn, T. S. Lawrence, and R. K. Ten Haken. "Improvement of CT-based treatment-planning models of abdominal targets using static exhale imaging." International Journal of Radiation Oncology, Biology, Physics 41(4), 939-943 (1998).

[109] E. De Ponti, S. Morzenti, L. Guerra, C. Pasquali, M. Arosio, V. Bettinardi, A. Crespi, M. C. Gilardi, and C. Messa. "Performance measurements for the PET/CT Discovery-600 using NEMA NU 2-2007 standards." Medical Physics 38(2), 968-974 (2011).

[110] W. Lu, P. J. Parikh, J. P. Hubenschmidt, J. D. Bradley, and D. A. Low. "A comparison between amplitude sorting and phase-angle sorting using external respiratory measurement for 4D CT." Medical Physics 33(8), 2964-2974 (2006).

[111] M. Dawood, F. Büther, N. Lang, O. Schober, and K. P. Schäfers. "Respiratory gating in positron emission tomography: A quantitative comparison of different gating schemes." Medical Physics 34(7), 3067-3076 (2007).

[112] Y. Picard and C. J. Thompson. "Motion correction of PET images using multiple acquisition frames." IEEE Transactions on Medical Imaging 16(2), 137-144 (1997).

[113] R. R. Fulton, S. R. Meikle, S. Eberl, J. Pfeiffer, and C. J. Constable. "Correction for head movements in positron emission tomography using an optical motiontracking system." IEEE Transactions on Nuclear Science 49(1), 116-123 (2002).

[114] G. J. Klein and R. H. Huesman. "Four-dimensional processing of deformable cardiac PET data." Medical Image Analysis 6(1), 29-46 (2002).

[115] M. Dawood, N. Lang, X. Y. Jiang, and K. P. Schafers. "Lung motion correction on respiratory gated 3-D PET/CT images." IEEE Transactions on Medical Imaging 25(4), 476-485 (2006).

[116] X. Jin, T. Mulnix, C. M. Sandiego, and R. E. Carson. "Evaluation of framebased and event-by-event motion-correction methods for awake monkey brain PET imaging." Journal of Nuclear Medicine 55(2), 287-293 (2014). 
[117] L. Fin, P. Bailly, J. Daouk, and M.-E. Meyer. "Motion correction based on an appropriate system matrix for statistical reconstruction of respiratorycorrelated PET acquisitions." Computer Methods and Programs in Biomedicine 96(3), e1-e9 (2009).

[118] T. F. Li, B. Thorndyke, E. Schreibmann, Y. Yang, and L. Xing. "Model-based image reconstruction for four-dimensional PET." Medical Physics 33(5), 12881298 (2006).

[119] J. Qi and R. H. Huesman. "List mode reconstruction for PET with motion compensation: A simulation study." In "IEEE International Symposium on Biomedical Imaging," pages 413-416. IEEE (2002).

[120] F. Qiao, T. Pan, J. W. Clark Jr, and O. R. Mawlawi. "A motion-incorporated reconstruction method for gated PET studies." Physics in Medicine and Biology 51(15), 3769-3783 (2006).

[121] A. Rahmim, K. Dinelle, J. C. Cheng, M. A. Shilov, W. P. Segars, S. C. Lidstone, S. Blinder, O. G. Rousset, H. Vajihollahi, B. M. W. Tsui, D. F. Wong, and V. Sossi. "Accurate event-driven motion compensation in high-resolution PET incorporating scattered and random events." IEEE Transactions on Medical Imaging 27(8), 1018-1033 (2008).

[122] F. Lamare, A. Le Maitre, M. Dawood, K. P. Schaefers, P. Fernandez, O. E. Rimoldi, and D. Visvikis. "Evaluation of respiratory and cardiac motion correction schemes in dual gated PET/CT cardiac imaging." Medical Physics 41(7), 072504 (13pp.) (2014).

[123] F. Lamare, M. Carbayo, and T. Cresson. "List-mode-based reconstruction for respiratory motion correction in PET using non-rigid body transformations." Physics in Medicine and Biology 52(17), 5187-5204 (2007).

[124] A. A. Isola, M. Grass, and W. J. Niessen. "Fully automatic nonrigid registrationbased local motion estimation for motion-corrected iterative cardiac CT reconstruction." Medical Physics 37(3), 1093-1109 (2010).

[125] C. Chan, X. Jin, E. K. Fung, M. Naganawa, T. Mulnix, R. E. Carson, and C. Liu. "Event-by-event respiratory motion correction for PET with 3D internal-1D external motion correlation." Medical Physics 40(11), 112507 (13pp.) (2013). 
[126] C. Liu, A. M. Alessio, and P. E. Kinahan. "Respiratory motion correction for quantitative PET/CT using all detected events with internal-external motion correlation." Medical Physics 38(5), 2715-2723 (2011).

[127] W. F. Jones. "Real-time event stream correction for patient motion in clinical 3-D PET." In "2001 IEEE Nuclear Science Symposium Conference Record," pages 2062-2064. IEEE (2002).

[128] R. Fulton, L. Tellmann, U. Pietrzyk, O. Winz, I. Stangier, I. Nickel, A. Schmid, S. Meikle, and H. Herzog. "Accuracy of motion correction methods for PET brain imaging." In "IEEE Symposium Conference Record Nuclear Science 2004.", pages 4226-4230. IEEE (2004).

[129] A. Rahmim, P. M. Bloomfield, M. Lenox, C. Michel, K. R. Buckley, T. J. Ruth, and V. Sossi. "Motion compensation in histogram-mode and list-mode EM reconstructions: beyond the event-driven approach." IEEE Transactions on Nuclear Science 51(5), 2588-2596 (2004).

[130] M. Menke, M. S. Atkins, and K. R. Buckley. "Compensation methods for head motion detected during PET imaging." IEEE Transactions on Nuclear Science 43(1), 310-317 (1996).

[131] P. M. Bloomfield, T. J. Spinks, J. Reed, L. Schnorr, A. M. Westrip, L. Livieratos, R. Fulton, and T. Jones. "The design and implementation of a motion correction scheme for neurological PET." Physics in Medicine and Biology 48(8), 959-978 (2003).

[132] X. Jin, T. Mulnix, J.-D. Gallezot, and R. E. Carson. "Evaluation of motion correction methods in human brain PET imaging: A simulation study based on human motion data." Medical Physics 40(10), 102503 (12pp.) (2013).

[133] K. E. Barratt. Ganong's Review of Medical Physiology. McGraw Hill Medical, 23rd edition (2010).

[134] W. Lu, M. M. Nystrom, P. J. Parikh, D. R. Fooshee, J. P. Hubenschmidt, J. D. Bradley, and D. A. Low. "A semi-automatic method for peak and valley detection in free-breathing respiratory waveforms." Medical Physics 33(10), 3634-3636 (2006).

[135] E. Rietzel and G. T. Y. Chen. "Improving retrospective sorting of 4D computed tomography data." Medical Physics 33(2), 377-379 (2006). 
[136] G. Chang, T. Chang, T. Pan, J. W. Clark, and O. R. Mawlawi. "Implementation of an automated respiratory amplitude gating technique for PET/CT: clinical evaluation." Journal of Nuclear Medicine 51(1), 16-24 (2010).

[137] S. S. Jani, C. G. Robinson, M. Dahlbom, B. M. White, D. H. Thomas, S. Gaudio, D. A. Low, and J. M. Lamb. "A comparison of amplitude-based and phase-based positron emission tomography gating algorithms for segmentation of internal target volumes of tumors subject to respiratory motion." International Journal of Radiation Oncology, Biology, Physics 87(3), 562-569 (2013).

[138] B.-K. Teo, B. Saboury, R. Munbodh, J. Scheuermann, D. A. Torigian, H. Zaidi, and A. Alavi. "The effect of breathing irregularities on quantitative accuracy of respiratory gated PET/CT." Medical Physics 39(12), 7390-7397 (2012).

[139] S. A. Nehmeh, Y. E. Erdi, C. C. Ling, K. E. Rosenzweig, O. D. Squire, L. E. Braban, E. Ford, K. Sidhu, G. S. Mageras, S. M. Larson, and J. L. Humm. "Effect of respiratory gating on reducing lung motion artifacts in PET imaging of lung cancer." Medical Physics 29(3), 366-371 (2002).

[140] M. Dawood, F. Büther, L. Stegger, X. Jiang, O. Schober, M. Schäfers, and K. P. Schäfers. "Optimal number of respiratory gates in positron emission tomography: A cardiac patient study." Medical Physics 36(5), 1775-1784 (2009).

[141] K. McLeish, D. Hill, D. Atkinson, J. M. Blackall, and R. Razavi. "A study of the motion and deformation of the heart due to respiration." IEEE Transactions on Medical Imaging 21(9), 1142-1150 (2002).

[142] T. Mäkelä, P. Clarysse, O. Sipilä, N. Pauna, Q. C. Pham, T. Katila, and I. E. Magnin. "A review of cardiac image registration methods." IEEE Transactions on Medical Imaging 21(9), 1011-1021 (2002).

[143] Q. Tang, J. Cammin, S. Srivastava, and K. Taguchi. "Four-dimensional nonrigid cardiac motion estimation." Proceedings of SPIE: Medical Imaging 2012: Image Processing 8314, 831454 (7pp.) (2012).

[144] P. Buhler, U. Just, E. Will, J. Kotzerke, and J. van den Hoff. "An accurate method for correction of head movement in PET." IEEE Transactions on Medical Imaging 23(9), 1176-1185 (2004).

[145] L. Livieratos, L. Stegger, P. M. Bloomfield, K. Schäfers, D. L. Bailey, and P. G. Camici. "Rigid-body transformation of list-mode projection data for respiratory 
motion correction in cardiac PET." Physics in Medicine and Biology 50(14), 3313-3322 (2005).

[146] J. Qi and R. H. Huesman. "Correction of motion in PET using event-based rebinning method: Pitfall and solution." Journal of Nuclear Medicine 43(5), 146-146 (2002).

[147] K. Thielemans, S. Mustafovic, and L. Schnorr. "Image reconstruction of motion corrected sinograms." In "IEEE Nuclear Science Symposium Conference Record," pages 2401-2406. IEEE (2003).

[148] P. J. Keall, V. R. Kini, S. S. Vedam, and R. Mohan. "Motion adaptive x-ray therapy: a feasibility study." Physics in Medicine and Biology 46(1), 1-10 (2001).

[149] P. J. Keall, E. Colvill, R. O’Brien, J. A. Ng, P. R. Poulsen, T. Eade, A. Kneebone, and J. T. Booth. "The first clinical implementation of electromagnetic transponder-guided MLC tracking." Medical Physics 41(2), 020702 (5pp.) (2014). 